UNIVERSIDADE DE SÃO PAULO

ESCOLA DE ENGENHARIA DE SÃO CARLOS

DEPARTAMENTO DE ENGENHARIA ELÉTRICA E DE COMPUTAÇÃO

PROGRAMA DE PÓS-GRADUAÇÃO EM ENGENHARIA ELÉTRICA

DESENVOLVIMENTO DE ESTRATÉGIAS DE OTIMIZAÇÃO CONTÍNUA E DISCRETA PARA PROBLEMAS DE FLUXO DE POTÊNCIA ÓTIMO

ANA PAULA MAZZINI

São Carlos

2016 

ANA PAULA MAZZINI

\section{DESENVOLVIMENTO DE ESTRATÉGIAS DE OTIMIZAÇÃO CONTÍNUA E DISCRETA PARA PROBLEMAS DE FLUXO DE POTÊNCIA ÓTIMO}

Tese apresentada à Escola de Engenharia de São Carlos da Universidade de São Paulo como parte dos requisitos para obtenção do título de Doutor em Ciências, Programa de Engenharia Elétrica.

Área de Concentração: Sistemas Elétricos de Potência.

Orientador: Prof. Dr. Eduardo Nobuhiro Asada.

São Carlos

2016

Trata-se da versão corrigida da tese. A versão original se encontra disponível na EESC/USP, que aloja o Programa de Pós-Graduação de Engenharia Elétrica. 
AUTORIZO A REPRODUÇÃO TOTAL OU PARCIAL DESTE TRABALHO, POR QUALQUER MEIO CONVENCIONAL OU ELETRÔNICO, PARA FINS DE ESTUDO E PESQUISA, DESDE QUE CITADA A FONTE.

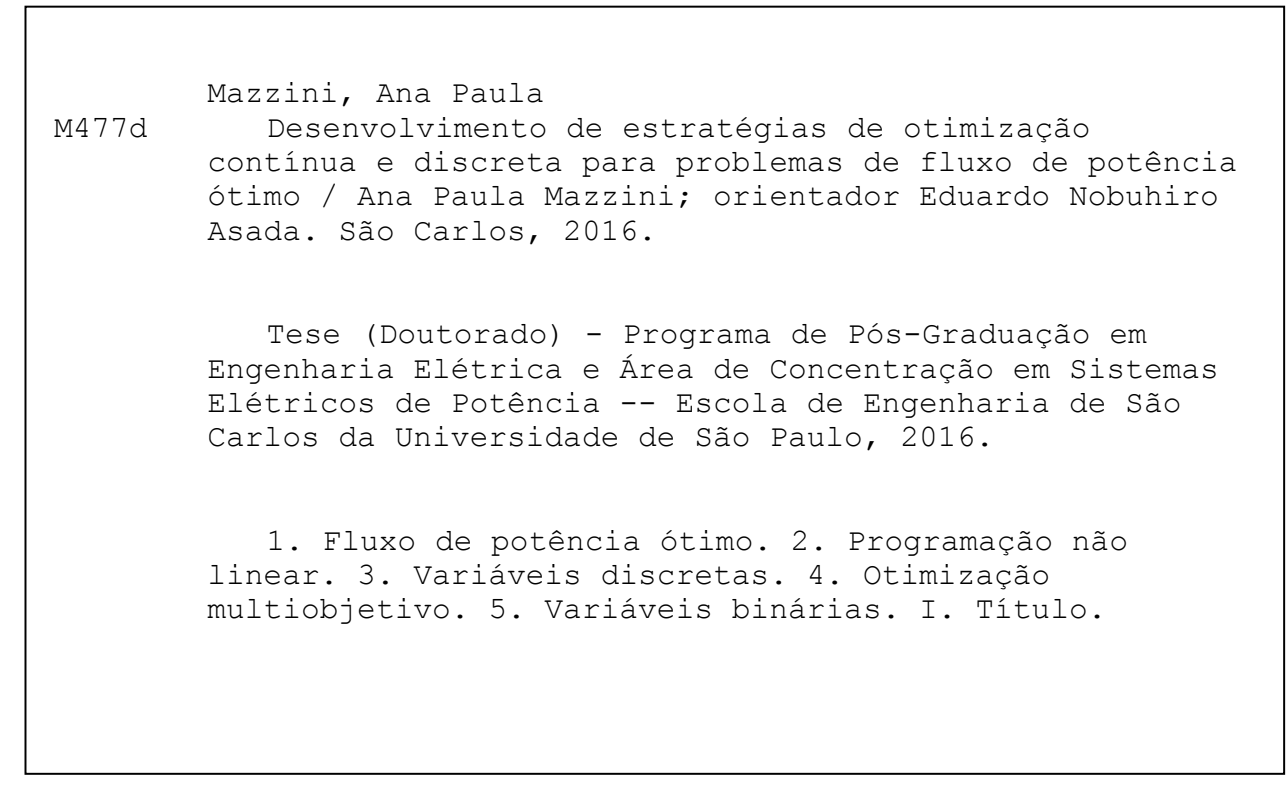




\section{FOLHA DE JULGAMENTO}

Candidata: Licenciada ANA PAULA MAZZINI.

Título da tese: "Desenvolvimento de estratégias de otimização contínua e discreta para problemas de fluxo de potência ótimo".

Data da defesa: 01/04/2016

Comissão Julgadora:

Resultado:

Prof. Associado Eduardo Nobuhiro Asada (Orientador) APROVADA (Escola de Engenharia de São Carlos/EESC)

Prof. Dr. Marcos Julio Rider Flores

APROVADO

(Universidade Estadual de Campinas/UNICAMP)

Prof. Associado Luis Fernando Costa Alberto

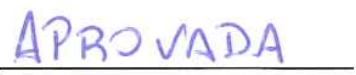

(Escola de Engenharia de São Carlos/EESC)

Prof. Titular Geraldo Roberto Martins da Costa

Emarada (Escola de Engenharia de São Carlos/EESC)

Prof. Dr. Rubén Augusto Romero Lázaro

aprivado

(Universidade Estadual Paulista "Júlio de Mesquita Filho"/UNESP - Ilha Solteira)

Coordenador do Programa de Pós-Graduação em Engenharia Elétrica: Prof. Associado Luis Fernando Costa Alberto

Presidente da Comissão de Pós-Graduação:

Prof. Associado Paulo César Lima Segantine 

A minha Família e ao meu esposo Filipe. 



\section{Agradecimentos}

Ao Professor Dr. Eduardo Nobuhiro Asada pelos ensinamentos, paciência, orientação e amizade durante todo o desenvolvimento deste trabalho.

Ao Professor Dr. Guilherme Guimarães Lage por toda paciência e ajuda durante meu doutorado e pela amizade desenvolvida ao longo destes anos.

A toda minha família pelos muitos sacrifícios feitos ao longo desta caminhada e por estarem ao meu lado em todos os momentos da minha vida.

Ao meu esposo Filipe por entender os sacrifícios exigidos por uma vida acadêmica.

Aos meus amigos do Laboratório de Análise de Sistemas de Energia Elétrica (LASEE) e a todos os amigos que estiveram presentes nesta caminhada.

Aos professores e funcionários do Departamento de Engenharia Elétrica (SEL) da Escola de Engenharia de São Carlos (EESC).

A CAPES pelo suporte financeiro durante o doutorado. 

A mente que se abre a uma nova ideia jamais voltará ao seu tamanho original.

Albert Einstein 



\section{Resumo}

MAZZINI, A. P. Desenvolvimento de estratégias de otimização contínua e discreta para problemas de fluxo de potência ótimo. 2016. 188 f. Tese (Doutorado em Ciências, Programa de Engenharia Elétrica) - Escola de Engenharia de São Carlos, Universidade de São Paulo, São Carlos, 2016.

O objetivo do presente trabalho é a investigação e o desenvolvimento de estratégias de otimização contínua e discreta para problemas de Fluxo de Potência Ótimo (FPO), onde existe a necessidade de se considerar as variáveis de controle associadas aos taps de transformadores em-fase e chaveamentos de bancos de capacitores e reatores shunt como variáveis discretas e existe a necessidade da limitação, e/ou até mesmo a minimização do número de ações de controle. Neste trabalho, o problema de FPO será abordado por meio de três estratégias. Na primeira proposta, o problema de FPO é modelado como um problema de Programação Não Linear com Variáveis Contínuas e Discretas (PNLCD) para a minimização de perdas ativas na transmissão; são propostas três abordagens utilizando funções de discretização para o tratamento das variáveis discretas. Na segunda proposta, considera-se que o problema de FPO, com os taps de transformadores discretos e bancos de capacitores e reatores shunts fixos, possui uma limitação no número de ações de controles; variáveis binárias associadas ao número de ações de controles são tratadas por uma função quadrática. Na terceira proposta, o problema de FPO é modelado como um problema de Otimização Multiobjetivo. O método da soma ponderada e o método $\epsilon$-restrito são utilizados para modificar os problemas multiobjetivos propostos em problemas mono-objetivos. As variáveis binárias associadas às ações de controles são tratadas por duas funções, uma sigmoidal e uma polinomial. Para verificar a eficácia e a robustez dos modelos e algoritmos desenvolvidos serão realizados testes com os sistemas elétricos IEEE de 14, 30, 57, 118 e 300 barras. Todos os algoritmos e modelos foram implementados em General Algebraic Modeling System (GAMS) e os solvers CONOPT, IPOPT, KNITRO e DICOPT foram utilizados na resolução dos problemas. Os resultados obtidos confirmam que as estratégias de discretização são eficientes e as propostas de modelagem para variáveis binárias permitem encontrar soluções factíveis para os problemas envolvendo as ações de controles enquanto os solvers DICOPT e KNITRO utilizados para modelar variáveis binárias não encontram soluções.

Palavras-chave: Fluxo de potência ótimo, Programação não linear, Variáveis discretas, Otimização multiobjetivo, Variáveis binárias. 



\section{Abstract}

MAZZINI, A. P. Development of continuous and discrete optimization strategies to problems of optimal power flow. 2016. 188 f. Tese (Doutorado em Ciências, Programa de Engenharia Elétrica) - Escola de Engenharia de São Carlos, Universidade de São Paulo, São Carlos, 2016.

The aims of this study are the investigation and the development of continuous and discrete optimization strategies to Optimal Power Flow (OPF) problems, where the control variables are the tap ratios of on-load tap changing (OLTC) transformers and shunt susceptances of switchable capacitors and reactors banks. These controls are discrete variables and a need for the limitation and/or even the minimization of the number of control adjustments is required. In this work, three strategies for solving the OPF problem have been deviced. In the first strategy, the OPF problem is modeled as a nonlinear programming problem with continuous and discrete variables for active power losses minimization; Three approaches using discretization functions for handling discrete variables have been investigated. In the second proposal, the OPF problem with discrete OLTC transformers and continuous shunt susceptances of switchable capacitors and reactors banks has a limitation on the number of control adjustments; binary variables associated with control adjustments are handled by a quadratic function. In the third proposal, the OPF problem is modeled as a multiobjective optimization problem. The weighting method and the $\epsilon$-constraint method are used to modify the proposed multiobjective problems onto single-objective problems. The binary variables associated with the controls are handled by sigmoidal and polynomial functions. The efficiency and robustness of the models and algorithms are shown for IEEE benchmark test-systems with up to 300 buses. All algorithms and models were implemented in GAMS modeling language and the results are obtained by means of CONOPT, IPOPT, KNITRO and DICOPT solvers. The results confirm that the discretization strategies are efficient and the proposed modeling for binary variables allows finding feasible solutions to problems involving the of controls while DICOPT and KNITRO solvers used to handle binary variables fail to find solutions.

Keywords: Optimal power flow, Nonlinear programming, Discrete variables, Multiobjective optimization, Binary variables. 



\section{Lista de Figuras}

Figura 3.1 - Representação gráfica da função (3.7) . . . . . . . . . . . . . . . 59

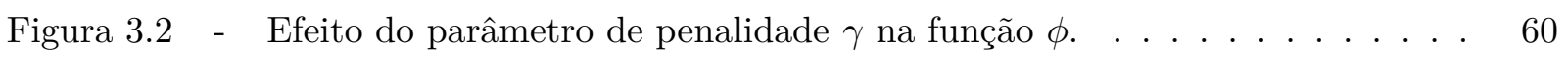

Figura 3.3 - Representação gráfica da função (3.12) com diferentes valores de $\beta_{1}$. . . . 63

Figura 3.4 - Representação gráfica da função $(3.12) \operatorname{com} \beta_{1}=10$. . . . . . . . . . . . 64

Figura 3.5 - Representação gráfica da função (3.12) com $\beta_{1}=10$ na proximidade do valor discreto $\mathrm{d} 2 \ldots \ldots \ldots \ldots$. . . . . . . . . . . . . . . . . 6 64

Figura 3.6 - Efeito do parâmetro de penalidade $\nu$ na função $\psi \ldots \ldots$. . . . . . . 65

Figura 3.7 - Representação gráfica do problema (3.15), onde $x \in \mathcal{D} \ldots \ldots \ldots$. . . . . 66

Figura 3.8 - Representação gráfica do problema (3.16), com $\beta_{1}=1$ e $\nu=0.005 \ldots$. . . 66

Figura 3.9 - Representação gráfica do problema (3.16), com $\beta_{1}=1$ e $\nu=0.05 . \quad \ldots \quad 67$

Figura 3.10 - Representação gráfica do problema (3.16), com $\beta_{1}=1$ e $\nu=0.1$. . . . . 67

Figura 3.11 - Representação gráfica do problema (3.16),com $\mathcal{D}_{1}=$ $\{-1,-0.75,-0.5,0.5\}, \beta_{1}=1$ e $\nu=0.005 \ldots \ldots \ldots 68$

Figura 3.12 - Representação gráfica do problema (3.16), com $\mathcal{D}_{1}=$ $\{-1,-0.75,-0.5,0.5\}, \beta_{1}=1$ e $\nu=0.05 \ldots \ldots \ldots 68 \ldots \ldots \ldots$

Figura 3.13 - Representação gráfica do problema (3.16), com $\mathcal{D}_{1}=$ $\{-1,-0.75,-0.5,0.5\}, \beta_{1}=1$ e $\nu=0.1 \ldots \ldots \ldots \ldots$

Figura 3.14 - Representação gráfica da função (3.25), para diferentes valores de $\tau$. . . . 73

Figura 3.15 - Representação gráfica da restrição (3.32) . . . . . . . . . . . . . . . 76

Figura 4.1 - Os conjuntos S e Z e as soluções ótimas de Pareto. . . . . . . . . . . . . 79

Figura 5.1 - Representação do Algoritmo 7. . . . . . . . . . . . . . . . . . 96

Figura 6.1 - Tap discreto para o modelo (2.5) os Algoritmos 1, 2 e 3 considerando a carga leve. . . . . . . . . . . . . . . . . . 107

Figura 6.2 - Tap discreto para o modelo (2.5) os Algoritmos 1, 2 e 3 considerando a carga média. . . . . . . . . . . . . . . 107

Figura 6.3 - Tap discreto para o modelo (2.5) os Algoritmos 1, 2 e 3 considerando a carga pesada. . . . . . . . . . . . . . 108

Figura 6.4 - Tap contínuo para o sistema IEEE de 118 barras pelo modelo (2.6) considerando a carga pesada. . . . . . . . . . . . . . . 111 
Figura 6.5 - Tap discreto para o sistema IEEE de 118 barras considerando a carga pesada.111

Figura 6.6 - Shunt contínuo para o sistema IEEE de 118 barras pelo modelo (2.6) considerando a carga pesada. . . . . . . . . . . . . . . . . . . 112

Figura 6.7 - Shunt discreto para o sistema IEEE de 118 barras considerando a carga pesada. . . . . . . . . . . . . . . . . . . 112

Figura 6.8 - Tap contínuo para o sistema IEEE de 300 barras pelo modelo (2.6) considerando a carga pesada. . . . . . . . . . . . . 115

Figura 6.9 - Tap discreto para o sistema IEEE de 300 barras considerando a carga pesada.115

Figura 6.10 - Shunt contínuo para o sistema IEEE de 300 barras pelo modelo (2.6) considerando a carga pesada. . . . . . . . . . . . . . . . 116

Figura 6.11 - Shunt discreto para o sistema IEEE de 300 barras considerando a carga pesada. . . . . . . . . . . . . . . . . 116

Figura 6.12 - Valor Inicial, Número de ações de Controle Ilimitados (NCI) e Número de ações de Controle Limitados (NCL) para os taps do sistema de 57 Barras. 121

Figura 6.13 - Valor Inicial, NCI e NCL para os taps do sistema de 118 Barras. . . . . . 121

Figura 6.14 - Valor Inicial, NCI e NCL para os taps do sistema de 300 Barras. . . . . . 122

Figura 6.15 - Perdas ativas pelo número de ações de controle para o sistema IEEE de 14 barras (Método da Soma Ponderada). . . . . . . . . . . . . . . . . 130

Figura 6.16 - Perdas ativas pelo número de ações de controle para o sistema IEEE de 30 barras (Método da Soma Ponderada). . . . . . . . . . . . . . . . . . 131

Figura 6.17 - Perdas ativas pelo número de ações de controle para o sistema IEEE de 57 barras (Método da Soma Ponderada). . . . . . . . . . . . . . . . 131

Figura 6.18 - Perdas ativas pelo número de ações de controle para o sistema IEEE de 118 barras (Método da Soma Ponderada). . . . . . . . . . . . . . . 132

Figura 6.19 - Perdas ativas pelo número de ações de controle pelo solver CONOPT e a função sigmoidal para o tratamento das variáveis binárias para o sistema IEEE de 300 barras (Método da Soma Ponderada). . . . . . . . . . . . . . 132

Figura 6.20 - Ações de Controle $\times \omega$ (Método da Soma Ponderada) . . . . . . . . . . . . 133

Figura 6.21 - Perdas Ativas $\times \omega$ (Método da Soma Ponderada). . . . . . . . . . . . 133

Figura 6.22 - Perdas ativas pelo número de ações de controle da curva combinada do sistema de 57 barras. Soluções não dominadas geradas pela combinação das soluções dos três solvers (Método da Soma Ponderada). . . . . . . . . . 134

Figura 6.23 - Perdas ativas pelo número de ações de controle da curva combinada do sistema de 118 barras. Soluções não dominadas geradas pela combinação das soluções dos três solvers (Método da Soma Ponderada). . . . . . . . . 135

Figura 6.24 - Perdas ativas pelo número de ações de controle da curva combinada do sistema de 300 barras. Soluções não dominadas geradas pela combinação das soluções de KNITRO e CONOPT (Método da Soma Ponderada). . .

Figura 6.25 - Perdas ativas pelo número de ações de controle para o sistema de 14 barras com todos os solvers (Método $\epsilon$-Restrito). . . . . . . . . . . . . 138 
Figura 6.26 - Perdas ativas pelo número de ações de controle para o sistema de 30 barras com todos os solvers (Método $\epsilon$-Restrito). . . . . . . . . . . . . . .

Figura 6.27 - Perdas ativas pelo número de ações de controle para o sistema de 57 barras com todos os solvers (Método $\epsilon$-Restrito). . . . . . . . . . . . . . .

Figura 6.28 - Perdas ativas pelo número de ações de controle para o sistema de 118 barras com todos os solvers (Método $\epsilon$-Restrito). . . . . . . . . . . . .

Figura 6.29 - Perdas ativas pelo número de ações de controle para o sistema de 300 barras com todos os solvers (Método $\epsilon$-Restrito). . . . . . . . . . . . . . 144

Figura 6.30 - Perdas ativas pelo número de ações de controle da curva combinada do sistema de 14 barras. Soluções não dominadas geradas pela combinação das soluções dos três solvers (Método $\epsilon$-Restrito). . . . . . . . . . . . . .

Figura 6.31 - Perdas ativas pelo número de ações de controle da curva combinada do sistema de 57 barras. Soluções não dominadas geradas pela combinação das soluções dos três solvers (Método $\epsilon$-Restrito). . . . . . . . . . . . . .

Figura 6.32 - Perdas ativas pelo número de ações de controle da curva combinada do sistema de 118 barras. Soluções não dominadas geradas pela combinação das soluções dos três solvers (Método $\epsilon$-Restrito). . . . . . . . . . . . . . 146

Figura 6.33 - Perdas ativas pelo número de ações de controle da curva combinada do sistema de 300 barras. Soluções não dominadas geradas pela combinação das soluções dos três solvers (Método $\epsilon$-Restrito). . . . . . . . . . . . . .

Figura 6.34 - Perdas ativas pelo número de ações de controle da curva combinada do sistema de 14 barras entre a Soma Ponderada e o $\epsilon$-Restrito. . . . . . . . .

Figura 6.35 - Perdas ativas pelo número de ações de controle da curva combinada do sistema de 30 barras entre a Soma Ponderada e o $\epsilon$-Restrito. . . . . . . . .

Figura 6.36 - Perdas ativas pelo número de ações de controle da curva combinada do sistema de 57 barras entre a Soma Ponderada e o $\epsilon$-Restrito. . . . . . . .

Figura 6.37 - Perdas ativas pelo número de ações de controle da curva combinada do sistema de 118 barras entre a Soma Ponderada e o $\epsilon$-Restrito. . . . . . . .

Figura 6.38 - Perdas ativas pelo número de ações de controle da curva combinada do sistema de 300 barras entre a Soma Ponderada e o $\epsilon$-Restrito. . . . . . . 150

Figura 6.39 - Trade-off entre as perdas ativas e o número de ações de controle para o sistema IEEE de 14 barras. . . . . . . . . . . . . . . . . . . 155

Figura 6.40 - Trade-off entre as perdas ativas e o número de ações de controle para o sistema IEEE de 30 barras. . . . . . . . . . . . . . . . . . . . 156

Figura 6.41 - Trade-off entre as perdas ativas e o número de ações de controle para o sistema IEEE de 57 barras. . . . . . . . . . . . . . . . . . 156

Figura 6.42 - Trade-off entre as perdas ativas e o número de ações de controle para o sistema IEEE de 118 barras. . . . . . . . . . . . . . . . . 157

Figura 6.43 - Trade-off entre as perdas ativas e o número de ações de controle para o sistema IEEE de 300 barras. . . . . . . . . . . . . . . . . . 157 
Figura 6.44 - Perdas ativas pelo número de ações de controle para o sistema de 118 barras. Soluções não dominadas geradas pela combinação das soluções das funções de discretização. . . . . . . . . . . . . . . . . . . . . . . . 160

Figura 6.45 - Perdas ativas pelo número de ações de controle para o sistema de 300 barras. Soluções não dominadas geradas pela combinação das soluções das funções de discretização. . . . . . . . . . . . . . . . . . . . . . 161

Figura 6.46 - Perdas ativas pelo número de ações de controle para o sistema IEEE de 14 barras. . . . . . . . . . . . . . . . . . . . . . 168

Figura 6.47 - Perdas ativas pelo número de ações de controle para o sistema IEEE de 30 barras. . . . . . . . . . . . . . . . . . . . . 168

Figura 6.48 - Perdas ativas pelo número de ações de controle para o sistema IEEE de 57 barras. . . . . . . . . . . . . . . . . . . . . 169

Figura 6.49 - Perdas ativas pelo número de ações de controle para o sistema IEEE de 118 barras. . . . . . . . . . . . . . . . . . . . . 169

Figura 6.50 - Perdas ativas pelo número de ações de controle para o sistema IEEE de 300 barras. . . . . . . . . . . . . . . . . . . 170

Figura 6.51 - Perdas ativas pelo número de ações de controle para o sistema de 300 barras. Soluções não dominadas geradas pela combinação das soluções das funções de discretização. . . . . . . . . . . . . . . . . . . . . . . . 171 


\section{Lista de Tabelas}

Tabela 6.1 - Características dos Sistemas Elétricos . . . . . . . . . . . . . . . . . 101

Tabela 6.2 - Solução da relaxação contínua para as variáveis de controle do sistema IEEE de 14 barras obtidas utilizando o modelo (2.6) . . . . . . . . . . . 102

Tabela 6.3 - Solução do modelo (2.5) para o sistema IEEE de 14 barras pelo Algoritmo 1 (função senoidal). . . . . . . . . . . . . . . . . . . . . 102

Tabela 6.4 - Solução do modelo (2.5) para o sistema IEEE de 14 barras pelo Algoritmo 2 (função polinomial). . . . . . . . . . . . . . . . . . 103

Tabela 6.5 - Solução do modelo (2.5) para o sistema IEEE de 14 barras pelo Algoritmo 3 (funções senoidal e polinomial) . . . . . . . . . . . . . . . . . . 103

Tabela 6.6 - Solução da relaxação contínua para as variáveis de controle do sistema IEEE de 30 barras obtidos utilizando o modelo (2.6) . . . . . . . . . . . . 104

Tabela 6.7 - Solução do modelo (2.5) para o sistema IEEE de 30 barras pelos Algoritmos 1,2 e $3 \ldots \ldots \ldots \ldots \ldots \ldots \ldots$

Tabela 6.8 - Valores mínimo, máximo e discretos das susceptâncias e reatores shunts. . 106

Tabela 6.9 - Resultados para o sistema IEEE de 57 barras. . . . . . . . . . . . . . . . 106

Tabela 6.10 - Shunt discreto encontrado para o modelo (2.5) com os Algoritmos 1, 2 e 3 considerando as cargas leve, média e pesada. . . . . . . . . . . . . . . 108

Tabela 6.11 - Valores mínimo, máximo e discretos das susceptâncias e reatores shunts. . 109

Tabela 6.12 - Resultados para o sistema IEEE de 118 barras. . . . . . . . . . . . . . . . 110

Tabela 6.13 - Valores mínimo, máximo e discretos das susceptâncias e reatores shunts. . 113

Tabela 6.14 - Resultados para o sistema IEEE de 300 barras. . . . . . . . . . . . . . . . 114

Tabela 6.15 - Características dos Sistemas Elétricos . . . . . . . . . . . . . . . . . . 119

Tabela 6.16 - Resultados numéricos para todos os sistemas IEEE . . . . . . . . . . . . . 120

Tabela 6.17 - Resultados obtidos para os taps de transformadores do sistema IEEE de 14 Barras. . . . . . . . . . . . . . . . . . . . . . . 120

Tabela 6.18 - Resultados obtidos para os taps de transformadores do sistema IEEE de 30 Barras. . . . . . . . . . . . . . . . . . . . 120

Tabela 6.19 - Características dos Sistemas Elétricos . . . . . . . . . . . . . . . 126

Tabela 6.20 - Resultados do sistema IEEE de 14 barras para os diferentes valores de $\omega . \quad 127$

Tabela 6.21 - Resultados do sistema IEEE de 30 barras para os diferentes valores de $\omega . \quad 127$ 
Tabela 6.22 - Resultados do sistema IEEE de 57 barras para os diferentes valores de $\omega$. Soluções com * são dominadas pelas demais. . . . . . . . . . . . . . . 128

Tabela 6.23 - Resultados do sistema IEEE de 118 barras para os diferentes valores de $\omega$. Soluções com * são dominadas pelas demais. . . . . . . . . . . . . . . . 129

Tabela 6.24 - Resultados do sistema IEEE de 300 barras para os diferentes valores de $\omega$. Perdas são dadas em MW e o tempo em segundos. . . . . . . . . . . . . .

Tabela 6.25 - Número de ações de controle e perdas ativas para o sistema de 300 barras usando KNITRO. . . . . . . . . . . . . . . . . . . . . . . 136

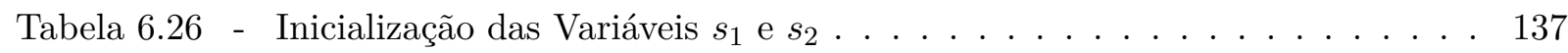

Tabela 6.27 - Resultados para o sistema IEEE de 14 barras com as diferentes estratégias. Soluções com * são dominadas pelas demais. . . . . . . . . . . . . . . 138

Tabela 6.28 - Resultados para o sistema IEEE de 30 barras com as diferentes estratégias. Soluções com * são dominadas pelas demais. . . . . . . . . . . . . . . . . 139

Tabela 6.29 - Resultados para o sistema IEEE de 57 barras. Soluções com * são dominadas pelas demais. . . . . . . . . . . . . . . . . . . . 140

Tabela 6.30 - Resultados para o sistema IEEE de 118 barras, somente com soluções não dominadas. . . . . . . . . . . . . . . . . . . . . 141

Tabela 6.31 - Resultados para o sistema IEEE de 300 barras, somente com soluções não dominadas. . . . . . . . . . . . . . . . . . . . 142

Tabela 6.32 - Características dos Sistemas Elétricos . . . . . . . . . . . . . 152

Tabela 6.33 - Resultados para o sistema IEEE de 14 barras. Soluções com * são dominadas pelas demais. . . . . . . . . . . . . . . 153

Tabela 6.34 - Resultados para o sistema IEEE de 30 barras. Soluções com * são dominadas pelas demais. . . . . . . . . . . . . . . . 153

Tabela 6.35 - Resultados para o sistema IEEE de 57 barras. Soluções com * são dominadas pelas demais. . . . . . . . . . . . . . . . . 154

Tabela 6.36 - Resultados para o sistema IEEE de 118 barras. Somente com soluções não dominadas. . . . . . . . . . . . . . . . . . . . 155

Tabela 6.37 - Resultados para o sistema IEEE de 300 barras. Somente com soluções não dominadas. . . . . . . . . . . . . . . . . . . . 158

Tabela 6.38 - Tensões e taps de transformadores para os $\epsilon$ 's 7 e 8 do sistema IEEE de 14 barras. . . . . . . . . . . . . . . . . . . . 159

Tabela 6.39 - Características dos Sistemas Elétricos . . . . . . . . . . . . . 162

Tabela 6.40 - Resultados do sistema IEEE de 14 barras para os diferentes valores de $\omega$ usando a SIGMOIDAL. . . . . . . . . . . . . . . . . . . . 163

Tabela 6.41 - Resultados do sistema IEEE de 30 barras para os diferentes valores de $\omega . \quad 164$

Tabela 6.42 - Resultados do sistema IEEE de 57 barras para os diferentes valores de $\omega . \quad 165$

Tabela 6.43 - Resultados do sistema IEEE de 118 barras para os diferentes valores de $\omega .166$

Tabela 6.44 - Resultados do sistema IEEE de 300 barras para os diferentes valores de $\omega .167$ 


\section{Lista de Siglas}

DE

FC

FPO

FPOR

GAMS

GRG

IA

KKT

MPI

NCI

NCL

OPF

PI

PIM

PL

PLIM

PLS
Despacho Econômico

Fluxo de Carga

Fluxo de Potência Ótimo

Fluxo de Potência Ótimo Reativo

General Algebraic Modeling System

Gradiente Reduzido Generalizado

Inteligência Artificial

Karush-Kuhn-Tucker

Método de Pontos Interiores

Número de ações de Controle Ilimitados

Número de ações de Controle Limitados

Optimal Power Flow

Programação Inteira

Programação Inteira-Mista

Programação Linear

Programação Linear Inteira-Mista

Programação Linear Sequencial 
PNL

PNLCD

PNLIM

PQ

PQS

PSD

SEE

SEP
Programação Não Linear

Programação Não Linear com Variáveis Contínuas e Discretas

Programação Não Linear Inteira-Mista

Programação Quadrática

Programação Quadrática Sequêncial

Programação Semi-Definida

Sistema de Energia Elétrica

Sistema Elétrico de Potência 


\section{Lista de Símbolos}

$\mathcal{B} \quad$ conjunto das barras do sistema

$\mathcal{G} \quad$ conjunto das barras de geração

$\mathcal{G}^{\prime} \quad$ conjunto das barras de geração, menos a barra slack

$\mathcal{C} \quad$ conjunto das barras de carga

$\mathcal{B}^{\text {sh }} \quad$ conjunto das barras com controle da magnitude de tensão por banco de capacitores

$\mathcal{L} \quad$ conjunto dos ramos $k-m$ que representam linhas de transmissão

$\mathcal{T} \quad$ conjunto dos ramos $k-m$ que representam transformadores em-fase com tap variável

$\mathcal{V}_{k} \quad$ conjunto das barras vizinhas à barra $k$

$V_{k} \quad$ magnitude da tensão na barra $k$

$V_{k}^{0} \quad$ valor inicial da magnitude da tensão na barra $k$

$V_{k}^{\text {min }} \quad$ limite inferior da magnitude da tensão na barra $k$

$V_{k}^{\max } \quad$ limite superior da magnitude da tensão na barra $k$

$\theta_{k m} \quad$ abertura angular entre os fasores das tensões nas barras $k$ e $m$

$P_{k m} \quad$ fluxo de potência ativa no ramo $k-m$

$Q_{k m} \quad$ fluxo de potência reativa no ramo $k-m$

$P_{k} \quad$ injeção líquida de potência ativa na barra $k$

$Q_{k} \quad$ injeção de potência reativa pelo shunt na barra $k$ 
$Q_{G_{k}} \quad$ potência reativa gerada na barra $k$

$Q_{G_{k}}^{\min } \quad$ limite inferior da potência reativa gerada na barra $k$

$Q_{G_{k}}^{\max } \quad$ limite superior da potência reativa gerada na barra $k$

$Q_{k}^{s h} \quad$ injeção líquida de potência reativa na barra $k$

$b_{k}^{s h} \quad$ susceptância equivalente do banco de capacitores e reatores da barra $k$

$b_{k}^{s h^{0}} \quad$ valor inicial do banco de capacitores e reatores da barra $k$

$b_{k}^{s h^{m i n}} \quad$ limite inferior para susceptância equivalente do banco de capacitores e reatores da barra $k$

$b_{k}^{s h^{\max }} \quad$ limite superior para susceptância equivalente do banco de capacitores e reatores da barra $k$

$b_{k}^{\prime} \quad$ valor discreto mais próximo de $b_{k}^{s h}$

$g_{k m} \quad$ condutância série do ramo $k-m$

$t_{k m} \quad$ tap do transformador em-fase do ramo $k-m$

$t_{k m}^{0} \quad$ valor inicial para o tap do transformador em-fase do ramo $k-m$

$t_{k m}^{m i n} \quad$ limite inferior do tap do ramo $k-m$

$t_{k m}^{\max } \quad$ limite superior do tap do ramo $k-m$

$t_{k m}^{\prime} \quad$ valor discreto mais próximo de $t_{k m}$

$\mathcal{D}_{k m}^{t a p} \quad$ conjunto dos valores discretos do tap do transformador em-fase do ramo $k-m$

$\mathcal{D}_{k}^{s h} \quad$ conjunto dos valores discretos da susceptância equivalente do banco de capacitores e reatores da barra $k$

$\operatorname{Bin}^{k} \quad$ é o vetor de variáveis binárias (0 ou 1), na qual cada componente $i$ de $B i n^{k}$ contém o binário mais próximo de cada componente $i$ de $\chi(y)^{k}$

$P_{\text {perdas }} \quad$ perdas ativas na transmissão 
$s_{1_{k}} \quad$ variável binária relacionada a tensão na barra $k$

$s_{2_{k m}} \quad$ variável binária relacionada ao tap de transformador do ramo $k-m$

$s_{3_{k}} \quad$ variável binária relacionada ao banco de capacitores e reatores da barra $k$

$N \quad$ número de variáveis de controles atuantes no sistema

$N^{\text {min }} \quad$ número mínimo de variáveis de controle que podem ser realocados para encontrar uma solução factível

$N^{\max } \quad$ número máximo de variáveis de controle que são realocados para encontrar uma solução ótima 



\section{Sumário}

\section{Capítulo 1}

Introdução $\quad 33$

1.1 Organização do trabalho . . . . . . . . . . . . . . . . . . . 36

\section{Capítulo 2}

O problema de Fluxo de Potência Ótimo $\quad 37$

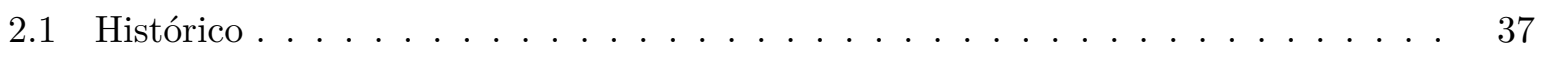

2.1.1 Variáveis de controle discretas . . . . . . . . . . . . . . . . 43

2.1.2 Limite do número de ações de controle . . . . . . . . . . . . . . . . 46

2.2 Modelos . . . . . . . . . . . . . . . . . . . . . . 48

2.2.1 Fluxo de Potencia Ótimo com minimização das perdas ativas na transmissão 49

2.2.2 Fluxo de Potencia Ótimo com limitação do Número de Ações de Controles 51

2.2.3 Fluxo de Potencia Ótimo com minimização do Número de Ações de Controles 53

2.2.4 Fluxo de Potencia Ótimo Multiobjetivo . . . . . . . . . . . . . . . . 55

\section{Capítulo 3}

Estratégias para o tratamento de variáveis discretas e binárias $\quad 57$

3.1 Função de discretização Senoidal . . . . . . . . . . . . . . . . . . . . . . . . . . . . . . . . . . .

$3.1 .1 \quad$ Definição da Constante $\alpha \ldots \ldots \ldots \ldots \ldots$. . . . . . . . . . 58

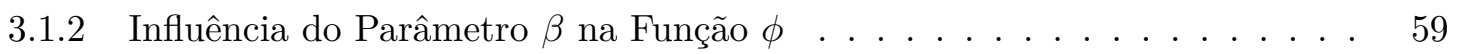

3.1.3 Influência do parâmetro de penalidade $\gamma$ na Função $\phi \ldots . . . .60$

3.1.4 Algoritmo de Discretização Utilizando a Função Senoidal . . . . . . . . . 60

3.2 Função de discretização Polinomial . . . . . . . . . . . . . . . . . . . . . 62

3.2.1 Influência do Parâmetro $\beta_{1}$ na Função $\psi$. . . . . . . . . . . . . . . . . . . 63

3.2.2 Influência do parâmetro de penalidade $\nu$ na Função $\psi$. . . . . . . . . . . 65

$3.2 .3 \quad$ Estimativa inicial para $\nu \ldots \ldots \ldots$. . . . . . . . . . . . . 69

3.2.4 Algoritmo Proposto Utilizando a Função Polinomial . . . . . . . . . . . . 70

3.3 Estratégia de Discretização Mista . . . . . . . . . . . . . . . . . 70

3.3.1 Algoritmo Proposto Utilizando a Estratégia de Discretização Mista . . . . 72

3.4 Função Sigmoidal . . . . . . . . . . . . . . . . . . . . . . . . . . 72

3.4.1 Algoritmo Proposto Utilizando a Função Sigmoidal . . . . . . . . . . . . . 74

3.5 Função Quadrática . . . . . . . . . . . . . . . . . . . . . . 75 


\section{Capítulo 4}

Otimização Multiobjetivo Não Linear $\quad \mathbf{7 7}$

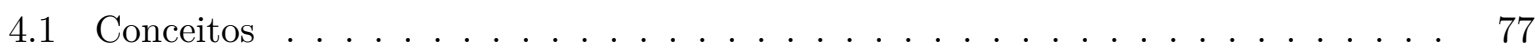

4.1 .1 Otimalidade de Pareto . . . . . . . . . . . . . . 78

4.1 .2 Tomando Decisões ． . . . . . . . . . . . . . . . . . . . . 78

4.2 Métodos de Otimização Multiobjetivo . . . . . . . . . . . . . . . . 79

4.2 .1 Métodos a-priori . . . . . . . . . . . . . . . . . . . . . . 80

4.2 .2 Métodos a-posteriori . . . . . . . . . . . . . . . . . . 80

4.2 .3 Métodos iterativos . . . . . . . . . . . . . . . . . . . . . . 80

4.3 Métodos Clássicos de Otimização Multiobjetivo . . . . . . . . . . . . . . . 80

4.3.1 Método da soma ponderada . . . . . . . . . . . . . . . . 80

4.3.1.1 Normalização . . . . . . . . . . . . . . . . . . . . . . . . . . 81

4.3 .2 Método $\epsilon$-restrito . . . . . . . . . . . . . . . . . . . . . . . . 81

\section{Capítulo 5}

Modelos e Algoritmos $\quad \mathbf{8 3}$

5.1 Limitação do Número de Ações de Controles em um problema de FPO . . . . . . 83

5.2 FPO Multiobjetivo com tap de transformadores contínuos e elementos shunt fixos 84

5.2 .1 Soma Ponderada . . . . . . . . . . . . . . . . . . . . . . 84

5.2 .2 Método $\epsilon$-restrito . . . . . . . . . . . . . . . . . . . . 87

5.3 FPO Multiobjetivo com tap de transformadores discretos e elementos shunt fixos modelado pelo método $\epsilon$-restrito f . . . . . . . . . . . . . . . . 88

5.4 FPO Multiobjetivo com tap de transformadores e elementos shunt discretos . . . 90

5.4.1 Algoritmos Propostos Utilizando a Função Sigmoidal e as Funções de Discretização . . . . . . . . . . . . . . . . . . . . . 9 94

5.4 .2 Considerações Finais . . . . . . . . . . . . . . . . . . . . . . . . . . 98

\section{Capítulo 6}

Resultados Numéricos $\quad 99$

6.1 Funções de discretização . . . . . . . . . . . . . . . . . . . . . . . . . . . . . 101

6.1.1 Sistema Elétrico IEEE de 14 Barras . . . . . . . . . . . . . . . . . . . . . 101

6.1 .2 Sistema Elétrico IEEE de 30 Barras . . . . . . . . . . . . . . . . . . . . 104

6.1.3 Sistema Elétrico IEEE de 57 Barras . . . . . . . . . . . . . . . . . . 106

6.1.4 Sistema Elétrico IEEE de 118 Barras . . . . . . . . . . . . . . . . . . . . . 109

6.1.5 Sistema Elétrico IEEE de 300 Barras . . . . . . . . . . . . . . . . . . . . . 113

6.1.6 Análise dos Resultados . . . . . . . . . . . . . . . . . . . . . 117

6.1 .7 Conclusões . . . . . . . . . . . . . . . . . . . . . . . . . 117

6.2 Limitação das Ações de Controles de um problema de FPO . . . . . . . . . . . . 119

6.2.1 Análise dos Resultados . . . . . . . . . . . . . . . . . . . . . . . . . 122

6.2 .2 Conclusões . . . . . . . . . . . . . . . . . . . . . . 122

6.3 FPO Multiobjetivo . . . . . . . . . . . . . . . . . . . . . . 124 
6.3.1 FPO Multiobjetivo com Tap de Transformadores Contínuos . . . . . . . 126

6.3.1.1 Método da Soma Ponderada . . . . . . . . . . . . . 126

6.3.1.2 Método $\epsilon$-Restrito . . . . . . . . . . . . . . . . . 137

6.3.1.3 Comparação entre Soma Ponderada e $\epsilon$-Restrito . . . . . . . . . 147

6.3.1.4 Conclusões . . . . . . . . . . . . . . . . . . . . 151

6.3.2 FPO Multiobjetivo com Tap de Transformadores Discretos . . . . . . . . 152

6.3.2.1 Análise dos Resultados . . . . . . . . . . . . . . . . . . 159

6.3.2.2 Conclusões . . . . . . . . . . . . . . . . 160

6.3.3 FPO Multiobjetivo com Tap de Transformadores e Susceptâncias Shunts

Discretos . . . . . . . . . . . . . . . . . . 162

6.3.3.1 Análise dos Resultados . . . . . . . . . . . . . . . . 170

6.3.3.2 Conclusões . . . . . . . . . . . . . . . . . . 171

6.3 .4 Conclusões Gerais . . . . . . . . . . . . . . . . . . . . . . . 172

\section{Capítulo 7}

$\begin{array}{ll}\text { Conclusões } & 173\end{array}$

7.1 Trabalhos Futuros . . . . . . . . . . . . . . . . . . 175

$\begin{array}{ll}\text { Referências Bibliográficas } & 177\end{array}$

Apêndice A

Solvers e Métodos $\quad 183$

A.1 CONOPT . . . . . . . . . . . . . . . . . . 183

A.1.1 Gradiente Reduzido Generalizado . . . . . . . . . . . . . . . . . . 184

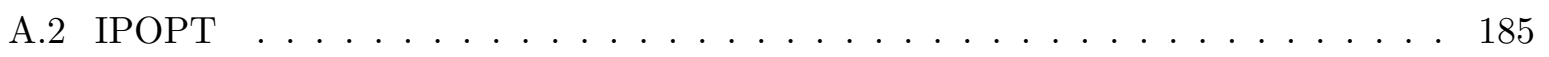

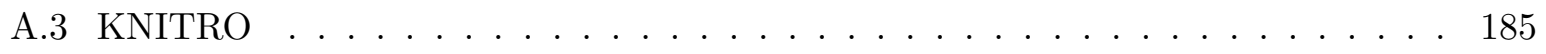

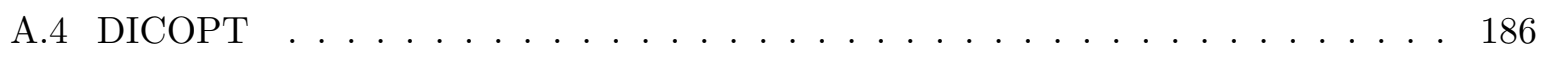

Apêndice B

Fluxo de Potência Ótimo - Expressões Gerais dos Fluxos 187 



\section{Capítulo 1}

\section{Introdução}

O problema de FPO é utilizado para analisar o planejamento e a operação dos Sistemas Elétricos de Potência (SEPs), podendo ser empregado para determinar diversos objetivos, como o despacho ótimo de potência ativa e reativa pelas unidades geradoras, o perfil ótimo de tensão ou mesmo a melhor distribuição de potência reativa. É um problema de otimização restrita, não linear, não convexo, estático, de grande porte, com variáveis contínuas e discretas, de difícil solução e de difícil aplicação real (on line) devido à presença de uma grande quantidade de variáveis discretas.

Desde sua proposição inicial por Carpentier (1962), o FPO tem se mostrado uma ferramenta essencial para o planejamento e a operação dos Sistemas de Energia Elétrica (SEEs). Atualmente, o FPO aparece como uma poderosa ferramenta para análise de SEEs graças ao desenvolvimento de metodologias eficientes (HUNEAULT; GALIANA, 1991), (MOMOH; EL-HAWARY; ADAPA, 1999a), (MOMOH; EL-HAWARY; ADAPA, 1999b), (CAPITANESCU et al., 2011), (FRANK; STEPONAVICE; REBENNACK, 2012a), (FRANK; STEPONAVICE; REBENNACK, 2012b) e à implementação de solvers robustos (QUINTANA; TORRES; MEDINA-PALOMO, 2000).

Um dos objetivos de um FPO é a determinação dos ajustes das variáveis de controle de um SEE responsáveis por otimizar um determinado desempenho operacional do sistema. Um exemplo é a resolução do problema de minimização das perdas ativas na transmissão, determina-se, portanto, o conjunto de ações de controle necessários para a minimização das perdas ativas.

Em 1988, Tinney et al. apresentaram um estudo sobre algumas deficiências encontradas em abordagens para problemas de FPO. Duas das deficiências citadas pelos autores são: 1) os métodos para lidar com variáveis discretas em modelos de FPO produzem soluções que têm custos significativamente elevados comparados com os custos produzidos pelas soluções ótimas; e 2) o número de ações de controle utilizados na resolução de problemas de FPO são demasiadamente grandes para serem executados nos sistemas reais. Os autores apresentaram uma discussão geralmente ignorada pela maioria dos trabalhos encontrados na literatura. Mais de duas décadas se passaram e algumas questões levantadas por (TINNEY et al., 1988) ainda não foram solucionadas. 
Com relação as variáveis discretas do problema de FPO, as variáveis de controle associadas aos taps de transformadores em-fase e chaveamentos de bancos de capacitores e reatores shunt são, na maioria das vezes, consideradas contínuas e, posteriormente, ajustadas, ou "arredondadas", para seu valor discreto mais próximo. Em problemas de FPO, autores sugerem que, essa abordagem pode descaracterizar a solução ótima do problema (LIU; PAPALEXOPOULOS; TINNEY, 1992) ou até resultar em soluções infactíveis (SOLER; ASADA; COSTA, 2013).

Problemas de PNLCD são geralmente resolvidos por métodos de Programação Inteira-Mista (PIM), entretanto, a complexidade computacional dos métodos clássicos para a determinação de uma solução ótima destes problemas tende a aumentar exponencialmente com o número de variáveis discretas (SOLER; ASADA; COSTA, 2013). Deste modo, a modelagem estritamente discreta de variáveis de controle e o uso de métodos de PIM inviabilizam formulações mais realistas de problemas de FPO para análise de SEEs de grande porte (LAGE, 2013). Tendo em vista estas considerações, Soler, Asada e Costa (2013) propuseram tratar as variáveis discretas do problema de Fluxo de Potência Ótimo Reativo (FPOR) como contínuas, descritas por funções senoidais incorporadas na função objetivo do problema original por um parâmetro de penalidade.

Com relação ao número de ações de controle utilizados na resolução de problemas de FPO, Capitanescu e Wehenkel (2011) propuseram abordagens para responder a questões em aberto essenciais no campo de FPO, inclusive a questão da limitação do número de ações de controles requeridas. Sua proposta consiste em introduzir às restrições de Fluxo de Carga (FC) uma restrição com variáveis binárias, onde cada variável binária é associada a um controle: se o controle atua a variável binária assume valor unitário; caso contrário, recebe valor nulo. Uma segunda restrição delimita o valor máximo da soma das variáveis binárias, restringindo a atuação das variáveis de controle.

O problema de FPO pode ser resolvido muitas vezes ao longo de um período de tempo e as soluções obtidas de uma resolução para outra podem implicar em pontos de operação totalmente diferentes. Isso significa que os operadores do sistema de transmissão podem ter dificuldade ou até não conseguir aplicar as mudanças no sistema em tempo real. Os modelos estudados neste trabalho visam representar o problema de FPO levando em consideração estas questões, como o caso das variáveis discretas e o número das ações de controles, de forma que os modelos possam ser utilizados para uma possível aplicação em centros de controle de energia elétrica.

O objetivo do presente trabalho é a investigação e o desenvolvimento de estratégias de otimização contínua e discreta para de problemas de FPO, onde existe a necessidade de se considerar as variáveis de controle associadas aos taps de transformadores em-fase e chaveamentos de bancos de capacitores e reatores shunt como variáveis discretas e existe a necessidade da limitação, e/ou até mesmo a minimização do número de ações de controle. Apesar da importância em se determinar a sequência ótima dos controles atuantes, este não é o foco deste documento e não será levado em consideração.

Neste trabalho, o problema de FPO será abordado por meio de três estratégias. Na primeira proposta, o problema de FPO é modelado como um problema de PNLCD para a 
minimização de perdas ativas na transmissão. As variáveis de controle associadas aos taps de transformadores e aos bancos de capacitores e reatores shunt são modeladas como discretas, onde os taps de transformadores são ajustados por passos iguais e os bancos de capacitores e reatores shunt são ajustados por passos diferentes. Para o tratamento destas variáveis discretas, são propostas três abordagens utilizando funções de discretização. Em todas as abordagens o problema de FPO é modificado de forma que as variáveis discretas são tratadas como contínuas por funções de discretização. Para a resolução do problema de FPO modificado utiliza-se o método do gradiente reduzido generalizado implementado no solver CONOPT (DRUD, 1985). Para verificar a eficiência desta abordagem utilizando as funções de discretização, três cenários de carregamento são considerados.

$\mathrm{Na}$ segunda proposta, considera-se que o problema de FPO, com os taps de transformadores discretos e bancos de capacitores e reatores shunts fixos, possui uma limitação no número de ações de controles. As variáveis binárias associadas ao número de ações de controles são tratadas por uma função quadrática e as variáveis discretas associadas aos taps de transformadores são tratadas por uma função polinomial.

$\mathrm{Na}$ terceira proposta, o problema de FPO é modelado como um problema de Otimização Multiobjetivo. Este problema de FPO multiobjetivo visa minimizar as perdas ativas na transmissão e minimizar as ações de controles, possuindo assim dois objetivos a serem considerados na função objetivo. Numa primeira etapa, as variáveis de controle associadas aos taps de transformadores são modeladas como contínuas enquanto as variáveis de controle associadas aos bancos de capacitores e reatores shunt são modeladas como fixas. Para cada variável de controle (tensões e taps de transformadores), associa-se uma variável binária que indica a atuação da variável de controle durante o processo de otimização. O problema de FPO multiobjetivo é tratado por dois métodos de otimização multiobjetivo, soma ponderada e $\epsilon$-restrito, ambos reescrevem o problema de modo que este passa a ser considerado um problema mono-objetivo. Utiliza-se uma função sigmoidal e uma função quadrática para tratamento das variáveis binárias.

Num segundo momento, as variáveis de controles taps de transformadores possam ser consideradas como discretas. Este novo problema multiobjetivo é modificado pelo método $\epsilon$-restrito e pela função quadrática para o tratamento das variáveis binárias. As funções de discretização polinomial e senoidal são utilizadas para modelar as variáveis discretas associadas aos taps de transformadores, o solver IPOPT e utilizado para resolver este problema. Num terceiro momento, ambos taps de transformadores e bancos de capacitores e reatores shunts são considerados discretos. O método da soma ponderada e a função sigmoidal para o tratamento das variáveis binárias são utilizados para modelar o problema multiobjetivo. As funções de discretização são utilizadas para a modelagem das variáveis discretas. Todos os testes foram implementados na interface para modelagem de problemas de programação matemática GAMS.

Para verificar a eficácia e a robustez das estratégias de discretização na resolução do problema de FPO modificado, da proposta de resolução do problema de FPO com limitação do 
número de ações de controle, e das abordagens de solução do problema de FPO multiobjetivo, serão realizados testes com os sistemas elétricos IEEE de 14, 30, 57, 118 e 300 barras.

Apresentamos neste trabalho a investigação e o desenvolvimento de estratégias para modelagem e resolução de problemas de FPO, onde existe a necessidade de se considerar as variáveis de controle associadas aos taps de transformadores em-fase e chaveamentos de bancos de capacitores e reatores shunt como variáveis discretas e existe a necessidade da limitação, e/ou até mesmo a minimização do número de ações de controle. Neste, utilizamos ferramentas de modelagem matemática a fim de modificar o problema de FPO, que consiste em um problema de PNLCD, e o problema de FPO multiobjetivo, aqui proposto, que contém variáveis binárias, em problemas de Programação Não Linear (PNL), os quais podemos aplicar métodos clássicos como Gradiente Reduzido Generalizado. Funções de discretização foram utilizadas para o tratamento de variáveis discretas e uma nova metodologia utilizando uma função sigmoidal foi proposta para o tratamento de variáveis binárias.

\subsection{Organização do trabalho}

No Capítulo 2 é apresentado o contexto histórico do problema de FPO, as abordagens que utilizam variáveis discretas, o histórico para o problema de FPO com limitação do número de ações de controles, e a modelagem destes problemas e do problema de FPO multiobjetivo com objetivos de minimizar as perdas ativas na transmissão e o número de ações de controle. No Capítulo 3 são propostas três estratégias para a discretização das variáveis de controles discretas utilizando funções de discretização. A primeira estratégia utiliza uma função senoidal para modelar todas as variáveis discretas do problema, a segunda utiliza uma função polinomial, e a terceira utiliza a função senoidal para modelar variáveis discretas que assumem valores em um conjunto periódico, enquanto a polinomial é utilizada para variáveis discretas não igualmente espaçadas. Também neste Capítulo, são apresentadas duas funções para o tratamento de variáveis binárias, uma função quadrática e uma função sigmoidal.

No Capítulo 4 descreve-se alguns conceitos básicos da Otimização Multiobjetivo Não Linear, como por exemplo, o conceito de Otimalidade de Pareto. Descreve-se também dois métodos clássicos para a resolução do problema multiobjetivo, o método da soma ponderada e o método $\epsilon$-restrito. No Capítulo 5 são apresentados os modelos propostos do problema multiobjetivo modificados pelos métodos da soma ponderada e $\epsilon$-restrito e pelas funções de discretização senoidal, polinomial e mista para o tratamento das variáveis discretas tap de transformadores e banco de capacitores e reatores shunt e pelas funções sigmoidal e quadrática de tratamento das variáveis binárias, relacionadas ao número de ações de controle. No Capítulo 6 são apresentados resultados para o problema de FPO com variáveis discretas, com limitação no número de ações de controle, e para o problema de FPO multiobjetivo contínuo e discreto. Por fim, no Capítulo 7 são apresentadas as considerações finais sobre o impacto do trabalho na literatura e as perspectivas futuras. 


\section{Capítulo 2}

\section{O problema de Fluxo de Potência Ótimo}

O objetivo deste capítulo é apresentar a evolução literária do problema de FPO e sua modelagem. A Seção 2.1 contém a revisão bibliográfica do problema de FPO e nas seções seguintes serão apresentadas algumas modelagens para o problema.

\subsection{Histórico}

Em 1962, Carpentier propôs uma formulação para o problema de Despacho Econômico (DE) incorporando equações de fluxo de potência em seu conjunto de restrições, utilizando uma função clássica Lagrangiana para transformar o problema de DE em um problema irrestrito. O método de Gauss-Seidel foi utilizado para resolver o problema sendo que sua solução é determinada quando as condições de Karush-Kuhn-Tucker (KKT) forem satisfeitas.Esta modelagem é considerada como propulsora dos estudos do problema de FPO.

Alguns anos mais tarde, Dommel e Tinney (1968) utilizaram o Método do Gradiente reduzido na resolução do FPO. Nesta abordagem, as restrições de desigualdade são tratadas por penalidades quadráticas incorporadas na função objetivo e as variáveis do problema são divididas em variáveis de controle e variáveis dependentes. Inicialmente, atribui-se valores para as variáveis de controle e as variáveis dependentes são calculadas resolvendo as equações de fluxo de potência pelo método de Newton. Calculam-se os multiplicadores de Lagrange associados às restrições de igualdades do problema e os ajustes das variáveis de controle são determinados pelo gradiente negativo da função objetivo. Utiliza-se o método da projeção para o tratamento das variáveis de controle cuja atualização implica na violação dos limites. Esse procedimento é repetido até que uma certa tolerância seja atendida.

Em 1969, Sasson apresentou uma abordagem baseada na associação dos métodos de (POWELL, 1964) e de (FLETCHER; POWELL, 1963), para a resolução de problemas de FPO. As restrições, igualdade e desigualdade, do problema são incorporadas na função objetivo por dois parâmetros distintos e em seguida o método de Fletcher-Powell minimiza a função de Powell. Nesta abordagem, utiliza-se a matriz Hessiana inversa e o gradiente da função de Powell para a 
atualização das variáveis. Uma deficiência deste método está no fato de que a matriz Hessiana inversa é cheia e pode ocasionar problemas de mau condicionamento na resolução de problemas de grande porte, podendo não convergir para uma solução.

Em Sasson, Viloria e Aboytes (1973) foi proposto o uso de um método de penalidade para a resolução dos problemas de FPO. Nesta abordagem, as restrições do problema são incorporadas à função objetivo por funções penalidades quadráticas, e o problema equivalente irrestrito é resolvido pelo método de Newton. A matriz Hessiana da função objetivo do problema irrestrito é calculada a cada iteração do método juntamente com a atualização de todas as variáveis, posteriormente verifica-se a convergência do método; caso a mesma não ocorra, os fatores de penalidade são aumentados e o processo se repete até que a tolerância exigida seja atingida. A matriz Hessiana da função auxiliar é esparsa, porém é diretamente influenciada pelo parâmetro de penalidade, podendo se tornar mal condicionada conforme este parâmetro cresça.

Rashed e Kelly (1974) propuseram uma abordagem de segunda ordem para a resolução de problemas de FPO utilizando o método do gradiente reduzido, visando superar algumas deficiências encontradas na abordagem proposta por Dommel e Tinney (1968), utiliza apenas uma barra de balanço, pode afetar todo desempenho da solução dependendo de sua localidade, e não utiliza restrições funcionais não lineares. Rashed e Kelly transformaram o problema de FPO em um problema irrestrito pelo método de multiplicadores de Lagrange, o estado da rede é determinado pela resolução do problema de FC, os multiplicadores de Lagrange associados às restrições são calculados e os ajustes das variáveis de controle são determinados pela matriz Hessiana da função Lagrangiana definida para estas variáveis. Se as variáveis de controle saem de seus limitantes quando atualizadas então estas são tratadas pelo método da projeção. Este processo continua até que a condição de convergência seja satisfeita.

Em 1982, Burchett, Happ e Wirgau propuseram uma abordagem de Programação Quadrática Sequêncial (PQS) baseada em modificar o problema original e resolver uma sequência de subproblemas linearmente restritos utilizando uma função objetivo tipo Lagrangiana aumentada. Uma característica fundamental deste algoritmo é que a solução converge quadraticamente nas restrições não lineares de fluxo de potência, em vez de ser obrigado a satisfazer as restrições ao longo do processo iterativo.

Sun et al. (1984) propuseram utilizar o método de Newton com Penalidade. Nesta abordagem o problema de FPO é transformado em um problema equivalente irrestrito, onde as restrições de igualdade e desigualdade são tratadas por multiplicadores de Lagrange incorporados na função objetivo, e os limites das variáveis de controle são tratados por Penalidades. As condições necessárias de primeira ordem são desacopladas em dois subproblemas pelo método de Newton, sendo que um subproblema é de potência ativa e o outro de potência reativa. A cada iteração, o método minimiza a função Lagrangiana aproximada por uma quadrática, e o ajuste das variáveis primais e duais da função Lagrangiana é feito simultaneamente. A solução para o problema é encontrada quando as condições necessárias de KKT são satisfeitas e as equações do FC estão dentro da tolerância exigida. 
Uma técnica de Programação Linear Sequencial (PLS) para o problema de despacho econômico reativo usando uma função de penalidade é proposta por (MOTA-PALOMINO; QUINTANA, 1986). Um conjunto de variáveis e o modelo de potência reativa do algoritmo de fluxo de carga desacoplado rápido são usados para derivar as sensibilidades lineares. Uma nova relaxação é proposta para lidar com o problema de Programação Linear (PL), com base em um gráfico de sensibilidade da potência reativa.

Santos Jr., Deckmann e Soares (1988) utilizaram um método de função Lagrangiana aumentada para a resolução de problemas de FPO. Nesta abordagem, o problema é transformado em um problema irrestrito através de uma função Lagrangiana clássica, tendo as restrições incorporadas a função objetivo por multiplicadores de Lagrange. Em seguida, essa função Lagrangiana é aumentada penalizando novamente as restrições do problema original por penalidades quadráticas. A função Lagrangiana aumentada é minimizada em relação as variáveis primais pelo método de Newton, os multiplicadores de Lagrange são atualizados de forma que a função Lagrangiana aumentada seja maximizada, e atualiza-se os parâmetros de penalidade de forma a garantir a existência de um ponto de sela dessa função Lagrangiana. Os autores afirmam que este método pode ser considerado como um aperfeiçoamento dos métodos propostos por Sasson, Viloria e Aboytes (1973) e Sun et al. (1984), já que evita o mau condicionamento da matriz Hessiana da função Lagrangiana aumentada.

Um estudo sobre abordagens de resolução de problemas de FPO foi apresentado por Huneault e Galiana (1991), contendo uma bibliografia de mais de trezentas publicações. Neste documento, os autores apresentam a evolução dos modelos de FPO e classificam os métodos existentes de acordo com suas técnicas de otimização. Afirmam, que apesar dos avanços existentes, existem problemas de mau condicionamento e difícil solução.

Granville (1994) propôs o algoritmo Primal-Dual barreira logarítmica na resolução do problema de FPOR. Neste método, são incorporadas variáveis de folga nas restrições de desigualdade do problema transformando-as em restrições de igualdade. Uma barreira logarítmica é inserida na função objetivo para garantir a não negatividade das variáveis de folga, tem-se ainda um parâmetro de barreira associado a esta função. Posteriormente as restrições de igualdades originais e transformadas são adicionadas a função objetivo por multiplicadores de Lagrange. O problema de barreira é resolvido pelo método de Newton, a cada iteração o método fornece direções de busca para a atualização das variáveis primais e duais do problema. A solução para o problema de FPOR é obtida quando todas as restrições forem satisfeitas e o parâmetro de barreira tender a zero. A escolha inicial do parâmetro de barreira influência na atuação do método.

Na mesma linha que Granville (1994), Wu, Debs e Marsten (1994) propuseram o algoritmo Primal-Dual barreira logarítmica na resolução do problema de FPO, mas utilizaram um método preditor-corretor variante do método de Newton-Raphson para resolução do problema de barreira. Estas modificações tornaram o desempenho desta estratégia melhor que o desempenho apresentado anteriormente. 
Torres e Quintana (1998) propuseram um Método de Pontos Interiores (MPI) utilizando coordenadas retangulares de tensão, ao invés de coordenadas polares, para resolver o problema de FPO. Com isso, o problema de FPO pode ser formulado por funções quadráticas, tornando a matriz Hessiana da função Lagrangiana constante. A estratégia utilizada para a resolução foi a mesma proposta por Granville (1994), os autores também apresentaram técnicas para a escolha do tamanho do passo na determinação das direções de busca, para a redução do fator de barreira e para o uso de um método preditor-corretor. Por fim, os autores concluíram que o desempenho do método com coordenadas retangulares se iguala com o método utilizando coordenadas polares.

Momoh, El-Hawary e Adapa (1999a, 1999b) apresentaram uma revisão bibliográfica sobre as abordagens de resolução de problemas de FPO, e classificaram essas abordagens em seis categorias:

- A PNL lida com problemas que envolvem funções objetivo e restrições não lineares. As restrições podem consistir em restrições de igualdade e / ou desigualdade. Métodos como minimização irrestrita sequencial e multiplicadores de Lagrange são considerados métodos de PNL.

- A Programação Quadrática (PQ) é uma forma especial de PNL, cuja função objetivo é quadrática e as restrições são lineares, considera-se nesta categoria métodos Quasi-Newton e métodos baseados em sensibilidade.

- Os métodos que consideram a solução das condições de KKT baseadas no método de Newton se referem em geral a equações que necessitam de processos iterativos de solução.

- A PL trata de problemas com restrições e função objetivo formulados como equações lineares onde as variáveis são não negativas. Os métodos mais utilizados neste tipo de problema são os métodos Simplex e Simplex revisado. A função objetivo e as equações do problema de FPO são linearizadas para permitir o uso de métodos de PL.

- Assim como a PNL, a Programação Inteira (PI) e PIM, exigem um grande esforço computacional e o número de variáveis discretas é um importante indicador da dificuldade em se solucionar um problema PIM. Existe na literatura uma mistura de técnicas de PL e PIM para resolver problemas típicos de FPO como o planejamento VAR. Os objetivos são lineares enquanto as restrições são lineares e não lineares com variáveis discretas ou inteiras.

- Por fim, métodos de pontos interiores inicialmente utilizados na resolução de problemas de PL.

Costa, Costa e Souza (2000) apresentaram um estudo com três abordagens baseadas no método de Newton, para a resolução de problemas de FPO. Os autores realizaram um estudo comparativo das principais característica de cada abordagem e suas metodologias. A primeira abordagem se refere a um método de penalidade juntamente com o método do conjunto ativo, a segunda abordagem se refere a um método primal-dual e a terceira a um método primal-dual 
com barreira logarítmica. Os critérios utilizados para comparação foram: perdas ativas na transmissão, geração de potência reativa na solução, número de iterações necessárias para a convergência e tempo de processamento. Ao final, os autores concluíram que cada método tem suas vantagens e desvantagens.

Em 2002, uma abordagem utilizando uma função Lagrangiana aumentada foi proposta por Costa para a resolução de problemas de FPOR. Neste caso, as condições necessárias de KKT são resolvidas pelo método de Newton modificado, as informações de segunda ordem do sistema original de equações são aproximadas e as informações de primeira ordem são mantidas intactas. De acordo com o autor, o método proposto requer menos memória computacional que os demais algoritmos disponíveis.

Sousa et al. (2003) propuseram uma nova abordagem para resolução do problema de FPO que melhora o desempenho do método de Newton. As restrições de desigualdades, referentes aos limites das magnitudes das tensões e aos taps dos transformadores, são associadas à função objetivo por meio de uma função penalidade; as restrições de desigualdade referentes às injeções de potência reativa são tratadas pelo método primal-dual barreira logarítmica; as restrições de igualdade do problema são incorporadas a função objetivo pela função Lagrangiana. O sistema gerado pelas condições necessárias de primeira ordem é resolvido pelo método de Newton e pela atualização dos termos de penalidade e de barreira.

Em 2004, Sousa, Baptista e Costa apresentaram uma abordagem para solução de problemas de FPO onde a função barreira clássica é substituída pela função barreira modificada na abordagem proposta por Granville (1994). As condições necessárias de primeira ordem são aplicadas ao problema Lagrangiano e este é resolvido pelo método de Newton, onde as melhores características dos métodos Lagrangianos e de barreira são combinadas.

Também em 2004, Rider et al. propuseram combinar MPIs para a resolução do problema de FPO, os métodos combinados foram: preditor-corretor, preditor com múltiplas correções e múltiplas correções centralizadas, pertencentes a família de MPI de alta ordem. Nesta abordagem, aproveitam-se as melhores propriedades de cada método para desenvolver um MPI mais robusto e com convergência mais rápida. De acordo com os autores, a ideia é diminuir o número de fatorações da matriz Hessiana da função Lagrangiana.

Baptista et al. (2006) apresentaram uma abordagem baseada na associação dos métodos de barreira e da função Lagrangiana aumentada. As restrições de caixa associadas aos limites das magnitudes das tensões e aos taps dos transformadores em-fase do problema de FPOR são desmembradas e transformadas em restrições de igualdade acrescentando variáveis de folga, a não negatividade das variáveis de folga é tratada pela função barreira logarítmica e as demais restrições do problema são tratadas pelo método da função Lagrangiana aumentada proposto por Santos Jr., Deckmann e Soares (1988). O problema de barreira-Lagrangiana aumentada é resolvido pelo método de Newton e sua solução é encontrada quando as condições de KKT forem satisfeitas.

Com objetivo de apresentar um estudo abrangente de vários métodos de otimização utilizados para resolver problemas de FPO, Pandya e Joshi (2008) discutiram a evolução de 
seis metodologias de resolução do problema de FPO. São elas: 1) Métodos de Programação Linear; 2) Métodos de Newton-Raphson; 3) Métodos de Programação Quadrática; 4) Métodos de Programação Não Linear; 5) Métodos de Pontos Interiores; e 6) Métodos de Inteligência Artificial (IA). Um enforque maior foi dados aos métodos de Inteligência Artificial abordando as técnicas: a) Redes Neurais Artificiais; b) Métodos de Lógica Fuzzy; c) Métodos de Algoritmo Genético; d) Sistema Inteligente e Busca Tabu Paralela; e) Programação Evolucionária; f) Colônia de formigas; e g) Enxame de Partículas.

Uma nova técnica usando Programação Semi-Definida (PSD) para o problema de FPO foi apresentada em Bai et al. (2008). O problema de FPO é reformulado como um problema de PSD e resolvido por um algoritmo de MPI. Essa reformulação do problema o transforma em um problema convexo, que pode ser resolvido por métodos de Pontos Interiores Primais-Duais que possuem convergência super-linear.

Uma abordagem que associa os métodos primal-dual barreira logarítmica e barreira modificada foi apresentada por Sousa, Baptista e Costa (2009). As restrições de caixa do problema de FPOR associadas aos limites das variáveis de controle do SEE são desmembradas em desigualdades, as desigualdades são transformadas em igualdades pelo acréscimo de variáveis de folga não negativas, e esta não negatividade é tratada pela função barreira logarítmica modificada. Uma função Lagrangiana é associada ao problema modificado e o problema Lagrangiano é resolvido pelo método de Newton.

Sousa, Baptista e Costa (2012) propuseram uma nova abordagem utilizando uma função Lagrangiana barreira modificada para resolver o problema de FPO. Nesta abordagem, as restrições de desigualdade são tratadas pelo método da função barreira modificada. De acordo com os autores, o método da função barreira modificada tem uma propriedade de convergência finita, isto é, a solução ótima pode realmente estar no limite do conjunto viável, e com isto as restrições de desigualdade podem ser nulas. É realizada uma análise comparativa do condicionamento numérico da matriz Hessiana, da abordagem utilizando uma função Lagrangiana barreira modificada, pela decomposição em valores singulares. A viabilidade da abordagem proposta também é demonstrada com testes comparativos para um MPI.

No mesmo ano, Frank, Steponavice e Rebennack (2012a, 2012b) apresentaram um levantamento bibliográfico em que pesquisaram tanto a literatura clássica quanto recente do problema de FPO. A pesquisa contribui com uma discussão abrangente de técnicas de otimização específicas que foram aplicadas para problema de FPO, com destaque para as vantagens, desvantagens e características computacionais de cada método. Este levantamento foi dividido em duas parte: Parte I) fornece uma introdução e examina os métodos de otimização determinísticos que foram aplicadas para problemas de FPO, entre eles métodos que utilizam gradiente, métodos de Newton, método Simplex, métodos de programação linear/quadrática sequencial, métodos de pontos interiores; e Parte II) examina a tendência recente de estocástica, ou métodos não determinísticos, técnicas de busca e métodos híbridos para problemas de FPO.

Molzahn et al. (2013) propuseram avanços de modelagem e de computação na aplicação da PSD ao problema de FPO de grande porte. Especificamente, uma relaxação da PSD do 
problema de FPO é apresentado incorporando múltiplos geradores à mesma barra e linhas paralelas. Três avanços de técnicas de decomposição existentes são apresentadas: um algoritmo de combinação que diminui ainda mais o tempo computacional, uma modificação de uma técnica de decomposição existente que se estende a sua aplicabilidade a redes gerais do sistema de potência, e um método para a obtenção do perfil ideal de tensão a partir da solução a uma decomposição da PSD.

\subsubsection{Variáveis de controle discretas}

As abordagens apresentadas a seguir, para a resolução de problemas de FPO, consideram as variáveis de controle do problema divididas em dois grupos, variáveis contínuas e variáveis discretas.

Liu, Papalexopoulos e Tinney (1992) apresentaram um algoritmo de discretização baseado no método de penalidade para determinar o ajuste ótimo das variáveis associadas aos bancos de capacitores e reatores shunt em problemas de FPO. Nesta abordagem, o problema de FPO é transformado em um problema irrestrito pelo método de multiplicadores de Lagrange, as variáveis discretas são tratadas por uma função penalidade incorporada a função objetivo por um parâmetro de penalidade. O algoritmo resolve uma sequência de problemas Lagrangianos pelo método de Newton, onde todas as variáveis são consideradas contínuas. A função penalidade usada para o tratamento das variáveis discretas é uma aproximação linear da função penalidade quadrática, por este motivo a matriz Hessiana da função Lagrangiana se torna fixa durante as iterações do método de Newton. O algoritmo converge quando as restrições forem satisfeitas e todas as variáveis de controle discretas assumirem valores discretos. Esta estratégia consiste em um método de arredondamento uma vez que no decorrer das iterações do método de Newton a penalidade obriga as variáveis se aproximarem de seus valores discretos.

Uma abordagem baseada em algoritmos genéticos para a resolução de problemas com variáveis de controle discretas é proposta por Bakirtzis et al. (2002). O algoritmo consiste em gerar uma população aleatória de soluções candidatas onde as variáveis de controle contínuas satisfazem seus limites e as discretas assumem valores discretos. Como essas soluções podem ser infactíveis ao considerar as demais restrições do problema, estas restrições são incluídas como penalidades na função aptidão que irá medir o potencial de cada solução candidata. Este algoritmo realiza as operações básicas de um algoritmo genético e posteriormente realiza operações avançadas específicas para o problema de FPO. Esta abordagem não garante que a solução encontrada é a solução ótima do problema original e o algoritmo requer um alto tempo computacional inviabilizando a utilização deste para problemas de grande porte.

No mesmo ano, Liu, Tso e Cheng apresentaram uma abordagem para o problema de FPOR de grande porte com variáveis de controle contínuas e discretas. Esta abordagem é baseada na incorporação de uma função penalidade em um algoritmo de pontos interiores primal-dual. A função penalidade utilizada é quadrática e não diferenciável nos pontos definidos como centros de vizinhança. Uma sequência de problemas de barreira-Lagrangiana é resolvida pelo método de Newton. Inicialmente assume-se que os parâmetros de penalidade de todas as variáveis são nulos, 
após a resolução pelo método de Newton, as variáveis de controle discretas que assumem valores discretos continuam com os parâmetros de penalidade nulos, e as demais tem estes parâmetros atualizados. Introduzir a penalidade no decorrer das iterações deste algoritmo afeta diretamente o sucesso desta abordagem, sendo que se a penalidade for introduzida antecipadamente as soluções podem ser não ótimas. A penalidade introduzida quando o algoritmo já convergiu para uma solução contínua, requer mais iterações do algoritmo para obter uma solução discreta. Os resultados obtidos na aplicação deste método mostram que as soluções obtidas são de boa qualidade em um tempo computacional aceitável.

Abidi et al. (2003) apresentaram a aplicação de um método de função Lagrangiana Aumentada barreira modificada para a solução de problemas de FPO com as variáveis taps de transformadores consideradas discretas. Inicialmente, os taps são considerados contínuos e o problema de FPO é resolvido pelo método da função Lagrangiana Aumentada barreira modificada. As restrições de desigualdade do problema são incorporadas a função Lagrangiana Aumentada barreira modificada pela função barreira modificada e as restrições de igualdade são incorporadas por uma função penalidade. Multiplicadores de Lagrange são associados a todas as restrições do problema. Deste modo, obtêm-se um problema de otimização irrestrito. Uma sequência destes problemas são resolvidos pelo método de Newton. Com este processo encontra-se uma solução contínua para o problema de FPO. Os taps são arredondados para seus valores discretos mais próximos e um novo problema de FPO é resolvido com os taps fixos em valores discretos. Este tipo de abordagem não garante que a solução encontrada é a solução ótima do problema e nem que uma solução será encontrada.

Uma abordagem utilizando um método de planos de corte para o tratamento das variáveis discretas taps de transformadores do problema de FPO foi proposta por Ding, Wang e Song (2004). Esta abordagem resolve uma sequência de linearizações do problema de FPO por um MPI. Inicialmente, o problema de FPO é resolvido com as variáveis discretas fixas, obtendo uma solução inicial factível. A partir desta etapa, o problema é linearizado na vizinhança da solução inicial. Se o problema linearizado for infactível, o processo é finalizado, caso contrário o problema de FPO linearizado é resolvido pelo MPI. Se na solução do problema linearizado algum tap assumir valor discreto, essa variável se torna fixa. Para os taps que não assumiram valores discretos na solução do problema linearizado é gerado um plano de corte, definindo assim um novo problema linearizado. Esse novo problema linearizado é novamente resolvido pelo MPI e o processo se repete até que todas as variáveis dos taps assumam valores discretos. Os resultados para esta abordagem foram encontrados em tempo menor que os resultados para abordagens de planos de corte que utilizam o método Simplex na resolução dos problemas linearizados.

Lin, Ho e Lin (2004) propuseram um algoritmo baseado em um procedimento de busca para o problema de FPO com variáveis de controle contínuas e discretas. Este algoritmo tem como objetivo fornecer uma solução discreta, não necessariamente ótima, em baixo tempo computacional. Inicialmente, resolve-se a relaxação contínua do problema de FPO e com esta solução cria-se um grande conjunto de soluções candidatas a partir de todas as possíveis combinações de arredondamento de cada variável. Uma heurística baseada no Teorema 
de Sensibilidade é utilizada para selecionar as potenciais soluções no conjunto de soluções candidatas, reduzindo assim o conjunto de busca. Em seguida, utiliza-se um modelo simplificado do problema de FPO para selecionar um subconjunto dessas potenciais soluções. Por fim, as soluções mais promissoras são avaliadas pelo modelo exato do problema de FPO, e segundo os autores, a solução encontrada é suficientemente boa para o problema de FPO com variáveis de controle contínuas e discretas.

Em 2007, AlRashidi e El-Hawary propuseram um algoritmo de otimização por Enxame de Partículas Híbrido para a resolução do problema de FPO com variáveis contínuas e discretas. Este algoritmo é uma combinação da otimização por Enxame de Partículas com o método de Newton-Raphson para minimizar o erro das equações de fluxo de potência. As equações de desigualdade do problema são tratadas por uma estratégia que preserva a factibilidade. É apresentado um estudo sobre os parâmetros utilizados neste algoritmo e segundo os autores o desempenho deste algoritmo depende do ajuste dos parâmetros utilizados e do critério a ser otimizado.

Um método de planos de corte melhorado foi apresentado por Liu, Cañizares e Huang (2009) para a resolução do problema de FPO com variáveis contínuas e discretas. Inicialmente, o método resolve o problema de FPO considerando todas as variáveis contínuas. Em seguida, o problema é linearizado na solução obtida e é resolvido por um MPI. Se na solução obtida as variáveis originalmente discretas assumem valores discretos, então a solução é considerada uma solução ótima, caso contrário, a base ótima é utilizada para gerar planos de corte que são adicionados ao problema linearizado. O novo problema linearizado é resolvido e o processo se repete até que uma solução discreta seja encontrada. Quando a solução ótima do problema linearizado é uma solução degenerada ou uma combinação convexa de soluções, falhas ocorrem ao gerar os planos de corte e devido a este motivo os autores propõem um método para resolver este caso. Apesar das melhoras o método de planos de corte proposto ainda necessita de um alto tempo computacional.

Capitanescu e Wehenkel (2010) propuseram três abordagem para o problema de FPO com variáveis de controle contínuas e discretas. A primeira consiste em resolver a relaxação do problema de FPO somente com variáveis contínuas por um MPI, em seguida, no ponto obtido é determinada a matriz sensibilidade da função lagrangiana em relação às variáveis discretas. Tais informações são incorporadas a um problema de Programação Linear Inteira-Mista (PLIM), que é resolvido pelo algoritmo de branch-and-cut, e cuja solução fornece os ajustes das variáveis de controle discretas do problema de FPO. Posteriormente, resolve-se o problema de FPO com as variáveis discretas fixas, obtendo assim o ajuste ótimo das variáveis de controle contínuas. A única diferença com relação à primeira abordagem, está na substituição do problema de PLIM para encontrar os ajustes das variáveis de controle discretas do problema de FPO por um procedimento baseado em uma função de mérito. A terceira abordagem explora os multiplicadores de Lagrange associados aos limites das variáveis de controle discretas. Os três procedimentos consistem de métodos iterativos que fixam valores discretos para as variáveis de controle discretas, afim de determinar a solução do problema de FPO e encontrar os valores das variáveis contínuas. As 
heurísticas para determinar os valores discretos exploram apenas as vizinhanças dos valores discretos da solução atual, dificilmente encontrando o ótimo global. Os resultados obtidos mostram que a segunda abordagem é melhor que as demais.

Soler, Asada e Costa (2013) propuseram uma função de discretização para o tratamento das variáveis de controle discretas do problema de FPO. Este método consiste em resolver uma sequência de problemas de PNL somente com variáveis contínuas. A função de discretização senoidal proposta é incorporada a função objetivo e penaliza as variáveis de controle quando estas não assumem valores discretos. A cada iteração o parâmetro de penalidade é atualizado e o processo termina quando as variáveis de controle inicialmente consideradas discretas assumem valores discretos. Os problemas de PNL somente com variáveis contínuas são resolvidos pelo método de Barreira-logaritmica primal-dual modificado. Os resultados obtidos foram comparados com os obtidos pelo solver comercial DICOPT e com uma estratégia de arredondamento. Os resultados mostram que esta abordagem é eficiente para o tratamento de variáveis de controle discretas e tem melhor resultado que as demais abordagens comparadas.

\subsubsection{Limite do número de ações de controle}

Algumas questões essenciais para os problemas de FPO são pouco abordadas na literatura devido a complexidade que elas empregam. Uma delas é a limitação do número de ações de controles permitidas em um FPO. Apresentamos nesta seção alguns estudos que levam em consideração esta questão.

Em 1988, Tinney et al. apresentaram o problema da limitação do número de ações de controles como uma das deficiências em um problema de FPO. Segundo os autores, a dificuldade de limitar o número de controles movidos num cálculo de FPO acontece devido ao fato de que a maioria das variáveis de controle participam tanto para melhorar o objetivo quanto para satisfazer as restrições, e as ações de controle não são fáceis de se classificar em termos de utilidade, pois a eficácia de uma ação não está necessariamente relacionada à sua quantidade de variação. Uma abordagem muito utilizada para limitar o número de ações de controle de um problema de FPO consiste na especificação dos controles autorizados a participar do cálculo do problema, tipicamente com base na experiência do operador e uma primeira execução do FPO onde todos os controles possíveis podem ser ajustados.

Soman, Parthasarathy e ThuKaram (1994) propuseram dois algoritmos para identificar o subconjunto mais eficaz das ações de controle e para minimizar essas ações. Um algoritmo visa minimizar o número de ações de controle enquanto, o outro algoritmo apenas limita o número de ações de controle por meio de uma restrição. Também é proposta uma função objetivo algorítmica para o tratamento da segurança e da economia, que elimina os ajustes de tentativa e erro de fatores ponderados de peso para um objetivo combinado. Um único parâmetro decide a prioridade entre o movimento dos controles e ganhos de segurança, bem como de redução de perdas. Os resultados demonstraram que o custo de movimentos do operador é um sub-ótimo da solução, e que a medida que os sistemas aumentam de tamanho, é impossível para o operador 
identificar o subconjunto mais eficaz de controles que resultam em ganhos máximos respeitando as restrições do mundo real.

Em 1996, Liu e Guan apresentam um método de conjuntos Fuzzy para a limitação do número de ações de controle de um FPO. É apresentada uma formulação Fuzzy do problema de FPO e aplicado a uma minimização dos custos de FPO. Como um primeiro passo, as restrições Fuzzy consideradas incluem o número total de ações de controle, bem como as restrições de fluxo nas linhas de transmissão. As restrições de limitação de ações de controle são relaxadas nas primeiras iterações. O objetivo é permitir que todas as variáveis de controle movam-se livremente, a fim de remover as violações causadas pelas restrições para chegar a um ponto viável sem considerar qualquer corte. Uma vez que a restrição de corte é introduzida, as variáveis de controle serão ajustada de acordo com a formulação Fuzzy em relação a posição inicial. Assim, as ações de controle local não ocorrem até que as inadequações de fluxo de potência sejam inferiores a uma certa tolerância. Os resultados dos testes em tempo real, mostram que os custos obtidos podem ser significativamente menores quando as violações dos limites operacionais normais são permitidas. Além disso, o número total de ações de controle pode ser razoavelmente reduzido com o aumento de custo ou pequenas violações de restrições suaves.

Capitanescu, Rosehart e Wehenkel (2009) apresentaram duas abordagens para o problema de FPO que não permitem mais do que um pré-determinado número de controles atuantes. A primeira abordagem consiste na reformulação do problema de Programação Não Linear Inteira-Mista (PNLIM) como um problema de programação matemática com restrições de equilíbrio. A segunda abordagem compreende, em adicionar ao problema clássico de FPO uma restrição não linear a qual se aproxima a restrição integrante da limitação do número de variáveis de controle que podem atuar. Ambas as abordagens são resolvidas por um MPI, adaptado às características particulares de cada abordagem. Para a primeira abordagem o algoritmo de pontos interiores utilizado encontrou muitos problemas numéricos quando o problema de FPO é altamente não linear.

Em 2011, Capitanescu e Wehenkel propuseram abordagens para responder a questões em aberto essenciais no campo de FPO: a limitação do número de ações de controles autorizadas; o trade-off entre a função objetivo e o número de ações de controles autorizadas; o cálculo do número mínimo de ações de controle necessários para satisfazer as restrições; e a determinação da sequência de ações de controle a serem tomadas pelo operador do sistema a fim de alcançar o seu objetivo de operação. As abordagens propostas dependem do cálculo de sensibilidades da função objetivo e das restrições de desigualdade no que diz respeito às ações de controle. Para o problema de limitar o número de ações de controle, é proposta uma restrição com variáveis binárias, onde cada variável binária é associada a um controle, se o controle atua a variável binária assume valor unitário, caso contrário recebe valor nulo. Uma segunda restrição restringe o valor máximo da soma das variáveis binárias, limitando assim a atuação das variáveis de controle. Na resolução do problema com variáveis binárias foi utilizado um algoritmo branch-and-cut. São propostas ainda duas variantes de um algoritmo que engloba todos as questões citadas acima, e os autores concluem que estas abordagens são compatíveis para operação em tempo real. 
Os avanços observados na literatura técnica proporcionaram um melhor entendimento do problema de limitação/minimização do número de ações de controles em problemas de FPO. Entretanto, apesar destes avanços as técnicas apresentadas não são eficientes no tratamento de problemas reais de grande porte. A seguir apresenta-se algumas modelagens para o problema de FPO, inclusive os casos onde considera-se o número de ações de controles. A modelagem das ações de controles é feita como proposta por (CAPITANESCU; WEHENKEL, 2011) utilizando as restrições de sensibilidade.

\subsection{Modelos}

Um problema de FPO é aquele que otimiza um determinado desempenho do sistema, sujeito ao balanço de potência ativa e reativa nas barras da rede, que são condições derivadas das leis de Tensão e Correntes de Kirchhoff, sujeito também aos limites de geração de potência reativa, e aos limites das variáveis de controle (LAGE, 2013).

O problema de FPO é utilizado para analisar a operação dos SEPs, podendo ser empregado para determinar diversos objetivos, como a distribuição ótima de potência pelas unidades geradoras, o perfil ótimo de tensão ou mesmo a melhor distribuição de potência reativa. É um problema de otimização restrita, não linear, não convexo, estático, com variáveis contínuas e discretas, e se torna de difícil solução e de difícil aplicação real (on line) a medida que o sistema aumenta de tamanho, devido à presença de uma grande quantidade de variáveis discretas. Outra dificuldade em termos de aplicações reais, é que o FPO deve ser particularizado para atender operações que são específicas de cada área, operador, país, etc.

Pode ser representado através de um problema PNLCD com restrições de igualdade e desigualdade como:

$$
\begin{array}{lll}
\min & f(x) & \\
\text { s.a: } & g_{i}(x)=0 & i=1, \cdots, p \\
& h_{i}(x) \leq 0 & i=1, \cdots, q \\
& x_{1_{i}}^{\text {min }} \leq x_{1_{i}} \leq x_{1_{i}}^{\max } & i=1, \cdots, m_{1} \\
& x_{2_{i}} \in \mathcal{D}_{x_{i}} & i=1, \cdots, m_{2}
\end{array}
$$

onde $x_{1} \in \mathbb{R}^{m_{1}}$ é o vetor das variáveis (de controles e dependentes) contínuas do sistema, $x_{2} \in \mathcal{D}_{x} \subset \mathbb{R}^{m_{2}}$ é o vetor das variáveis de controles discretas do sistema, sendo $x=\left(x_{1}, x_{2}\right) ; \mathcal{D}_{x_{i}}$ é o conjunto dos valores discretos que a variável $x_{2_{i}}$ pode assumir; $f: \mathbb{R}^{m_{1}} \times \mathbb{R}^{m_{2}} \rightarrow \mathbb{R}$ é uma função escalar que representa um determinado desempenho do sistema; $g: \mathbb{R}^{m_{1}} \times \mathbb{R}^{m_{2}} \rightarrow \mathbb{R}^{p}$, $\operatorname{com} p<m_{1}+m_{2}$ é o conjunto das restrições de igualdade formado pelas equações do problema de FC, pelas equações do modelo de atuação dos dispositivos de controle, etc; $h: \mathbb{R}^{m_{1}} \times \mathbb{R}^{m_{2}} \rightarrow \mathbb{R}^{q}$ é o conjunto das restrições de desigualdade formado pelos limites de geração de potência reativa, 
limites de fluxos de potência nas linhas de transmissão, etc; e $x_{1_{i}}^{\min }$ e $x_{1_{i}}^{\max }$ são os limites inferiores e superiores das variáveis $x_{1_{i}}$, respectivamente.

A maioria dos modelos encontrados na literatura desconsideram as variáveis $x_{2}$ como variáveis discretas, sendo estas tratadas como contínuas, devido a complexidade em se tratar as exigências impostas pelas variáveis discretas. Com isso, a restrição (2.1e) é reformulada da seguinte forma:

$$
x_{2_{i}}^{\min } \leq x_{2_{i}} \leq x_{2_{i}}^{\max }
$$

onde $x_{2_{i}}^{\min }$ e $x_{2_{i}}^{\max }$ são os limites inferiores e superiores da variável $x_{2_{i}}$, respectivamente, sendo $x_{2_{i}}^{\min }=\min \left\{\mathcal{D}_{x_{i}}\right\}$ e $x_{2_{i}}^{\max }=\max \left\{\mathcal{D}_{x_{i}}\right\}$.

$\mathrm{Na}$ sequência serão apresentados os modelos de FPO que nos propomos a estudar e desenvolver estratégias de resolução.

\subsubsection{Fluxo de Potencia Ótimo com minimização das perdas ativas na transmissão}

A seguir apresentam-se três modelagens para o problema de FPO: modelo contínuo; modelagem considerando variáveis contínuas e discretas; modelo relaxado.

O modelo contínuo do problema de FPO, visando minimizar as perdas ativas na transmissão, é muito comum na literatura. As variáveis de controle discretas associadas aos taps de transformadores são consideradas contínuas e as variáveis de controle discretas associadas aos shunt de barra são consideradas fixas. Assim, o modelo contínuo do problema de FPO com objetivo de minimizar as perdas ativas na transmissão é dado por:

$$
\begin{aligned}
& \text { min } \quad P_{\text {perdas }} \\
& \text { s.a: } \quad P_{k}-\sum_{m \in \mathcal{V}_{k}} P_{k m}(V, \theta, t)=0 \quad \forall k \in \mathcal{G}^{\prime} \cup \mathcal{C} \\
& Q_{k}+Q_{k}^{s h}\left(V_{k}\right)-\sum_{m \in \mathcal{V}_{k}} Q_{k m}(V, \theta, t)=0 \quad \forall k \in \mathcal{C} \\
& Q_{G_{k}}^{\min } \leq Q_{G_{k}}(V, \theta, t) \leq Q_{G_{k}}^{\max } \quad \forall k \in \mathcal{G} \\
& V_{k}^{\text {min }} \leq V_{k} \leq V_{k}^{\max } \quad \forall k \in \mathcal{B} \\
& t_{k m}^{\min } \leq t_{k m} \leq t_{k m}^{\max } \quad \forall k, m \in \mathcal{T}
\end{aligned}
$$

onde $P_{\text {perdas }}$ representa as perdas ativas na transmissão e é dado por:

$$
P_{\text {perdas }}=\sum_{k, m \in \mathcal{L} \cup \mathcal{T}} g_{k m}\left(\frac{1}{t_{k m}^{2}} V_{k}^{2}+V_{m}^{2}-2 \frac{1}{t_{k m}} V_{k} V_{m} \cos \theta_{k m}\right) ;
$$


$V$ e $\theta$ são, respectivamente, os vetores das magnitudes e ângulos de fase da tensão nas barras do sistema; $t$ é o vetor dos taps variáveis de transformadores em-fase; $P_{k}$ e $Q_{k}$ representam, respectivamente, as injeções líquidas de potência ativa e reativa na barra $k ; Q_{G_{k}}$ é a potência reativa gerada na barra $k ; Q_{G_{k}}^{\min }$ e $Q_{G_{k}}^{\max }$ representam a potência reativa mínima e máxima gerada na barra $k$, respectivamente; $Q_{k}^{s h}$ é a injeção de potência reativa pelo shunt da barra $k ; P_{k m}$ e $Q_{k m}$ são os fluxos de potência ativa e reativa no ramo $k-m ; \theta_{k m}$ é a diferença entre os ângulos de fase da tensão nas barras $k$ e $m ; g_{k m}$ é a condutância série associada ao elemento de transmissão do ramo $k-m ; V_{k}^{m i n}$ e $V_{k}^{\max }$ são os limites inferiores e superiores das tensões $V_{k}$, respectivamente; $t_{k m}^{\text {min }} \mathrm{e}$ $t_{k m}^{\max }$ são os limites inferiores e superiores das variáveis taps dos transformadores, respectivamente.

Ainda, tem-se que $\mathcal{B}$ é o conjunto de todas as barras do sistema; $\mathcal{G}$ é conjunto das barras de geração; $\mathcal{G}^{\prime}$ é conjunto das barras de geração menos a barra slack; $\mathcal{C}$ é conjunto das barras de carga; $\mathcal{L}$ é o conjunto dos ramos $k-m$ que representam linhas de transmissão; $\mathcal{T}$ é conjunto dos ramos $k$ - $m$ que representam transformadores em-fase com tap variável; $\mathcal{V}_{k}$ é o conjunto das barras vizinhas à barra $k$.

$\mathrm{Na}$ formulação do problema de FPO com variáveis contínuas e discretas, os taps dos transformadores e as susceptâncias equivalentes aos bancos de capacitores e reatores shunts são modelados como variáveis de controle discretas do sistema. Assim o modelo de FPO para minimização das perdas ativas na transmissão com variáveis contínuas e discretas é definido como:

$$
\begin{array}{lll}
\text { min } & P_{\text {perdas }} & \\
\text { s.a: } & P_{k}-\sum_{m \in \mathcal{V}_{k}} P_{k m}(V, \theta, t)=0 & \forall k \in \mathcal{G}^{\prime} \cup \mathcal{C} \\
& Q_{k}+Q_{k}^{s h}\left(V_{k}, b_{k}^{s h}\right)-\sum_{m \in \mathcal{V}_{k}} Q_{k m}(V, \theta, t)=0 & \forall k \in \mathcal{C} \\
& & \\
Q_{G_{k}}^{\text {min }} \leq Q_{G_{k}}\left(V, \theta, t, b_{k}^{s h}\right) \leq Q_{G_{k}}^{\max } & \forall k \in \mathcal{G} \\
V_{k}^{\text {min }} \leq V_{k} \leq V_{k}^{\text {max }} & \forall k \in \mathcal{B} \\
& t_{k m} \in \mathcal{D}_{k m}^{\text {tap }} & \forall k, m \in \mathcal{T} \\
& b_{k}^{s h} \in \mathcal{D}_{k}^{s h} & \forall k \in \mathcal{B}^{s h}
\end{array}
$$

onde $b^{\text {sh }}$ é o vetor das susceptâncias equivalentes dos bancos de capacitores e reatores shunt; $\mathcal{D}_{k m}^{t a p}$ é o conjunto dos valores discretos que os taps dos transformadores podem assumir; $\mathcal{D}_{k}^{s h}$ é o conjunto dos valores discretos que as susceptâncias equivalentes aos bancos de capacitores e reatores shunts podem assumir; e $\mathcal{B}^{\text {sh }}$ é conjunto das barras com controle da magnitude de tensão por bancos de capacitores e reatores shunt;

Dado o modelo (2.5) podemos definir um novo modelo contínuo o qual chamaremos de Relaxação Contínua para o modelo discreto, onde as variáveis taps dos transformadores e as susceptâncias equivalentes dos bancos de capacitores e reatores shunt são consideradas contínuas. A Relaxação Contínua (2.6) encontra um limitante inferior para a função objetivo do modelo 
(2.5). O propósito em definir este modelo é totalmente matemático e para análise dos resultados da Seção 6, já que desconsideramos que as susceptâncias equivalentes aos bancos de capacitores e reatores shunts nunca serão contínuas no modelo real.

$$
\begin{array}{lll}
\min & P_{\text {perdas }} & \\
\text { s.a: } & P_{k}-\sum_{m \in \mathcal{V}_{k}} P_{k m}(V, \theta, t)=0 & \forall k \in \mathcal{G}^{\prime} \cup \mathcal{C} \\
& Q_{k}+Q_{k}^{s h}\left(V_{k}, b_{k}^{\text {sh }}\right)-\sum_{m \in \mathcal{V}_{k}} Q_{k m}(V, \theta, t)=0 & \forall k \in \mathcal{C} \\
& Q_{G_{k}}^{\text {min }} \leq Q_{G_{k}}\left(V, \theta, t, b_{k}^{\text {sh }}\right) \leq Q_{G_{k}}^{\text {max }} & \forall k \in \mathcal{G} \\
V_{k}^{\text {min }} \leq V_{k} \leq V_{k}^{\text {max }} & \forall k \in \mathcal{B} \\
& t_{k m}^{\text {min }} \leq t_{k m} \leq t_{k m}^{\text {max }} & \forall k, m \in \mathcal{T} \\
& b_{k}^{\text {shin }} \leq b_{k}^{\text {sh }} \leq b_{k}^{\text {shax }} & \forall k \in \mathcal{B}^{\text {sh }}
\end{array}
$$

onde $b_{k}^{s h^{\text {min }}}$ e $b_{k}^{s h^{\max }}$ são os limitantes inferiores e superiores da susceptância equivalente dos bancos de capacitores e reatores shunt, respectivamente.

\subsubsection{Fluxo de Potencia Ótimo com limitação do Número de Ações de Controles}

Nesta seção, apresentamos os modelos contínuo e discreto para o problema de FPO, considerando a limitação do número de ações de controles. Restrições são acrescentadas em (2.2) e (2.6) para limitar o número de ações de controles como proposto em (CAPITANESCU; WEHENKEL, 2011). Os modelos resultantes são apresentados a seguir.

O modelo do problema de FPO com variáveis de controles contínuas tendo por objetivo minimizar as perdas ativas na transmissão com um número de ações de controles limitado é dado por:

$$
\begin{aligned}
& \min \quad P_{\text {perdas }} \\
& \text { s.a: } \quad P_{k}-\sum_{m \in \mathcal{V}_{k}} P_{k m}(V, \theta, t)=0 \quad \forall k \in \mathcal{G}^{\prime} \cup \mathcal{C} \\
& Q_{k}+Q_{k}^{s h}\left(V_{k}\right)-\sum_{m \in \mathcal{V}_{k}} Q_{k m}(V, \theta, t)=0 \quad \forall k \in \mathcal{C} \\
& Q_{G_{k}}^{\min } \leq Q_{G_{k}}(V, \theta, t) \leq Q_{G_{k}}^{\max } \quad \forall k \in \mathcal{G} \\
& V_{k}^{\min } \leq V_{k} \leq V_{k}^{\max } \quad \forall k \in \mathcal{B} \\
& t_{k m}^{\min } \leq t_{k m} \leq t_{k m}^{\max } \quad \forall k, m \in \mathcal{T} \\
& s_{1_{k}}\left(V_{k}^{\text {min }}-V_{k}^{0}\right) \leq\left(V_{k}-V_{k}^{0}\right) \leq s_{1_{k}}\left(V_{k}^{\max }-V_{k}^{0}\right) \quad \forall k \in \mathcal{G} \\
& s_{2_{k m}}\left(t_{k m}^{m i n}-t_{k m}^{0}\right) \leq\left(t_{k m}-t_{k m}^{0}\right) \leq s_{2_{k m}}\left(t_{k m}^{\max }-t_{k m}^{0}\right) \quad \forall k, m \in \mathcal{T} \\
& N^{\text {min }} \leq \sum_{k \in \mathcal{G}} s_{1_{k}}+\sum_{k, m \in \mathcal{T}} s_{2_{k m}} \leq N^{\max } \\
& s_{1_{k}} \in\{0,1\} \\
& s_{2_{k m}} \in\{0,1\}
\end{aligned}
$$


onde $s_{1_{k}}$ é a variável binária relacionada as tensões, e será 1 quando o controle da tensão da barra $k$ é ajustada com relação ao estado inicial, e 0 quando a tensão da barra $k$ não é ajustada; $s_{2_{k m}}$ é a variável binária relacionada aos taps de transformadores, e será 1 quando o tap do ramo $k-m$ atua, e 0 quando o tap do ramo $k-m$ não atua; $V_{k}^{0}$ é o valor inicial da tensão na barra $k ; t_{k m}^{0}$ é o valor inicial do tap do transformador no ramo $k-m ; N^{m i n}$ é o número mínimo de variáveis de controle que podem ser realocados para encontrar uma solução factível; $N^{\text {max }}$ é o número máximo de variáveis de controle que são realocados para encontrar uma solução ótima.

O modelo do problema de FPO com variáveis de controles contínuas e discretas, considerando o elemento shunt fixo, tendo por objetivo minimizar as perdas ativas na transmissão com um número de ações de controles limitado é dado por:

$$
\begin{aligned}
& \text { min } \quad P_{\text {perdas }} \\
& \text { s.a: } \quad P_{k}-\sum_{m \in \mathcal{V}_{k}} P_{k m}(V, \theta, t)=0 \quad \forall k \in \mathcal{G}^{\prime} \cup \mathcal{C} \\
& Q_{k}+Q_{k}^{s h}\left(V_{k}\right)-\sum_{m \in \mathcal{V}_{k}} Q_{k m}(V, \theta, t)=0 \quad \forall k \in \mathcal{C} \\
& Q_{G_{k}}^{\min } \leq Q_{G_{k}}(V, \theta, t) \leq Q_{G_{k}}^{\max } \quad \forall k \in \mathcal{G} \\
& V_{k}^{\min } \leq V_{k} \leq V_{k}^{\max } \quad \forall k \in \mathcal{B} \\
& s_{1_{k}}\left(V_{k}^{\text {min }}-V_{k}^{0}\right) \leq\left(V_{k}-V_{k}^{0}\right) \leq s_{1_{k}}\left(V_{k}^{\max }-V_{k}^{0}\right) \quad \forall k \in \mathcal{G} \\
& s_{2_{k m}}\left(t_{k m}^{\min }-t_{k m}^{0}\right) \leq\left(t_{k m}-t_{k m}^{0}\right) \leq s_{2_{k m}}\left(t_{k m}^{\max }-t_{k m}^{0}\right) \quad \forall k, m \in \mathcal{T} \\
& N^{\text {min }} \leq \sum_{k \in \mathcal{G}} s_{1_{k}}+\sum_{k, m \in \mathcal{T}} s_{2_{k m}} \leq N^{\max } \\
& t_{k m} \in \mathcal{D}_{k m}^{t a p} \\
& \forall k, m \in \mathcal{T} \\
& s_{1_{k}} \in\{0,1\} \\
& k \in \mathcal{G} \\
& s_{2_{k m}} \in\{0,1\}
\end{aligned}
$$

onde $\mathcal{D}_{k m}^{t a p}$ é o conjunto dos valores discretos no qual os taps dos transformadores podem assumir valores.

O modelo com variáveis de controles contínuas e discretas do problema de FPO considerando shunt tendo por objetivo de minimizar perdas levando em conta um limitado número de ações de controle é definido como: 


$$
\begin{aligned}
& \text { min } P_{\text {perdas }} \\
& \text { s.a: } \quad P_{k}-\sum_{m \in \mathcal{V}_{k}} P_{k m}(V, \theta, t)=0 \\
& Q_{k}+Q_{k}^{s h}\left(V_{k}, b_{k}^{s h}\right)-\sum_{m \in \mathcal{V}_{k}} Q_{k m}(V, \theta, t)=0 \quad \forall k \in \mathcal{C} \\
& Q_{G_{k}}^{\min } \leq Q_{G_{k}}\left(V, \theta, t, b_{k}^{s h}\right) \leq Q_{G_{k}}^{\max } \quad \forall k \in \mathcal{G} \\
& V_{k}^{\text {min }} \leq V_{k} \leq V_{k}^{\max } \quad \forall k \in \mathcal{B} \\
& s_{1_{k}}\left(V_{k}^{\text {min }}-V_{k}^{0}\right) \leq\left(V_{k}-V_{k}^{0}\right) \leq s_{1_{k}}\left(V_{k}^{\max }-V_{k}^{0}\right) \quad \forall k \in \mathcal{G} \\
& s_{2_{k m}}\left(t_{k m}^{\min }-t_{k m}^{0}\right) \leq\left(t_{k m}-t_{k m}^{0}\right) \leq s_{2_{k m}}\left(t_{k m}^{\max }-t_{k m}^{0}\right) \quad \forall k, m \in \mathcal{T} \\
& s_{3_{k}}\left(b_{k}^{s h^{m i n}}-b_{k}^{s h^{0}}\right) \leq\left(b_{k}^{s h}-b_{k}^{s h^{0}}\right) \leq s_{3_{k}}\left(b_{k}^{s h^{\max }}-b_{k}^{s h^{0}}\right) \quad \forall k \in \mathcal{B}^{s h} \\
& N^{\text {min }} \leq \sum_{k \in \mathcal{G}} s_{1_{k}}+\sum_{k, m \in \mathcal{T}} s_{2_{k m}}+\sum_{k \in \mathcal{B}^{s h}} s_{3_{k}} \leq N^{\max } \\
& t_{k m} \in \mathcal{D}_{k m}^{t a p} \\
& \forall k, m \in \mathcal{T} \\
& b_{k}^{s h} \in \mathcal{D}_{k}^{s h} \\
& \forall k \in \mathcal{B}^{\text {sh }} \\
& s_{1_{k}} \in\{0,1\} \\
& k \in \mathcal{G} \\
& s_{2_{k m}} \in\{0,1\} \\
& k, m \in \mathcal{T} \\
& s_{3_{k}} \in\{0,1\}
\end{aligned}
$$

onde $s_{3_{k}}$ é a variável binária relacionada aos elementos shunts, e será 1 quando o shunt da barra $k$ é ajustada com relação ao estado inicial, e 0 quando o shunt da barra $k$ não é ajustada.

\subsubsection{Fluxo de Potencia Ótimo com minimização do Número de Ações de Controles}

Nesta seção, apresentamos dois modelos para o problema de FPO onde o objetivo não é mais minimizar as perdas ativas na transmissão, e sim, minimizar o número de ações de controles realizados para encontrar uma solução factível. Os modelos apresentados a seguir serão apenas utilizados no desenvolvimento da formulação multiobjetivo do problema de FPO e não apresentamos resultados numéricos para eles.

O modelo de FPO com variáveis contínuas tendo por objetivo minimizar o número de ações de controles é dado por: 


$$
\begin{aligned}
& \min \quad N \\
& \text { s.a: } \quad P_{k}-\sum_{m \in \mathcal{V}_{k}} P_{k m}(V, \theta, t)=0 \\
& Q_{k}+Q_{k}^{s h}\left(V_{k}\right)-\sum_{m \in \mathcal{V}_{k}} Q_{k m}(V, \theta, t)=0 \quad \forall k \in \mathcal{C} \\
& Q_{G_{k}}^{\min } \leq Q_{G_{k}}(V, \theta, t) \leq Q_{G_{k}}^{\max } \quad \forall k \in \mathcal{G} \\
& V_{k}^{\text {min }} \leq V_{k} \leq V_{k}^{\max } \quad \forall k \in \mathcal{B} \\
& t_{k m}^{\min } \leq t_{k m} \leq t_{k m}^{\max } \quad \forall k, m \in \mathcal{T} \\
& s_{1_{k}}\left(V_{k}^{\min }-V_{k}^{0}\right) \leq\left(V_{k}-V_{k}^{0}\right) \leq s_{1_{k}}\left(V_{k}^{\max }-V_{k}^{0}\right) \quad \forall k \in \mathcal{G} \\
& s_{2_{k m}}\left(t_{k m}^{\min }-t_{k m}^{0}\right) \leq\left(t_{k m}-t_{k m}^{0}\right) \leq s_{2_{k m}}\left(t_{k m}^{\max }-t_{k m}^{0}\right) \quad \forall k, m \in \mathcal{T} \\
& s_{1_{k}} \in\{0,1\} \quad k \in \mathcal{G} \\
& s_{2_{k m}} \in\{0,1\} \quad k, m \in \mathcal{T}
\end{aligned}
$$

onde $N$ é o número de variáveis de controles atuantes no sistema, ou seja,

$$
N=\sum_{k \in \mathcal{G}} s_{1_{k}}+\sum_{k, m \in \mathcal{T}} s_{2_{k m}} .
$$

O modelo com variáveis de controles contínuas e discretas do problema de FPO tendo por objetivo minimizar o número de ações de controles é dado por:

$$
\begin{aligned}
& \min \quad N \\
& \text { s.a: } \quad P_{k}-\sum_{m \in \mathcal{V}_{k}} P_{k m}(V, \theta, t)=0 \\
& \forall k \in \mathcal{G}^{\prime} \cup \mathcal{C} \\
& Q_{k}+Q_{k}^{s h}\left(V_{k}, b_{k}^{s h}\right)-\sum_{m \in \mathcal{V}_{k}} Q_{k m}(V, \theta, t)=0 \quad \forall k \in \mathcal{C} \\
& Q_{G_{k}}^{\min } \leq Q_{G_{k}}\left(V, \theta, t, b_{k}^{s h}\right) \leq Q_{G_{k}}^{\max } \quad \forall k \in \mathcal{G} \\
& V_{k}^{\min } \leq V_{k} \leq V_{k}^{\max } \quad \forall k \in \mathcal{B} \\
& s_{1_{k}}\left(V_{k}^{\min }-V_{k}^{0}\right) \leq\left(V_{k}-V_{k}^{0}\right) \leq s_{1_{k}}\left(V_{k}^{\max }-V_{k}^{0}\right) \quad \forall k \in \mathcal{G} \\
& s_{2_{k m}}\left(t_{k m}^{\min }-t_{k m}^{0}\right) \leq\left(t_{k m}-t_{k m}^{0}\right) \leq s_{2_{k m}}\left(t_{k m}^{\max }-t_{k m}^{0}\right) \quad \forall k, m \in \mathcal{T} \\
& s_{3_{k}}\left(b_{k}^{s h^{\min }}-b_{k}^{s h^{0}}\right) \leq\left(b_{k}^{s h}-b_{k}^{s h^{0}}\right) \leq s_{3_{k}}\left(b_{k}^{s h^{\max }}-b_{k}^{s h^{0}}\right) \quad \forall k \in \mathcal{B}^{s h} \\
& t_{k m} \in \mathcal{D}_{k m}^{t a p} \quad \forall k, m \in \mathcal{T} \\
& b_{k}^{s h} \in \mathcal{D}_{k}^{s h} \quad \forall k \in \mathcal{B}^{s h} \\
& s_{1_{k}} \in\{0,1\} \quad k \in \mathcal{G} \\
& s_{2_{k m}} \in\{0,1\} \quad k, m \in \mathcal{T} \\
& s_{3_{k}} \in\{0,1\} \quad k \in \mathcal{B}^{s h}
\end{aligned}
$$


onde $N$ é o número de variáveis de controle atuantes no sistema e pode ser representado por:

$$
N=\sum_{k \in \mathcal{G}} s_{1_{k}}+\sum_{k, m \in \mathcal{T}} s_{2_{k m}}+\sum_{k \in \mathcal{B}^{s h}} s_{3_{k}}
$$

\subsubsection{Fluxo de Potencia Ótimo Multiobjetivo}

Nesta seção, apresentamos os modelos propostos para o problema de FPO com objetivos de minimizar as perdas ativas na transmissão e o número de ações de controles permitidos atuar, desta maneira, o problema de FPO passa a ter dois objetivos, deixando de ser um problema mono-objetivo.

O modelo multiobjetivo do problema de FPO com variáveis de controles contínuas e objetivos de minimizar tanto as perdas ativas na transmissão quanto o número de ações de controles é dado por:

$$
\begin{aligned}
& \min \quad\left[P_{\text {perdas }}, N\right]^{T} \\
& \text { s.a: } \quad P_{k}-\sum_{m \in \mathcal{V}_{k}} P_{k m}(V, \theta, t)=0 \quad \forall k \in \mathcal{G}^{\prime} \cup \mathcal{C} \\
& Q_{k}+Q_{k}^{s h}\left(V_{k}\right)-\sum_{m \in \mathcal{V}_{k}} Q_{k m}(V, \theta, t)=0 \quad \forall k \in \mathcal{C} \\
& Q_{G_{k}}^{\min } \leq Q_{G_{k}}(V, \theta, t) \leq Q_{G_{k}}^{\max } \quad \forall k \in \mathcal{G} \\
& V_{k}^{\text {min }} \leq V_{k} \leq V_{k}^{\text {max }} \quad \forall k \in \mathcal{B} \\
& t_{k m}^{\min } \leq t_{k m} \leq t_{k m}^{\max } \quad \forall k, m \in \mathcal{T} \\
& s_{1_{k}}\left(V_{k}^{\text {min }}-V_{k}^{0}\right) \leq\left(V_{k}-V_{k}^{0}\right) \leq s_{1_{k}}\left(V_{k}^{\text {max }}-V_{k}^{0}\right) \quad \forall k \in \mathcal{G} \\
& s_{2_{k m}}\left(t_{k m}^{m i n}-t_{k m}^{0}\right) \leq\left(t_{k m}-t_{k m}^{0}\right) \leq s_{2_{k m}}\left(t_{k m}^{\max }-t_{k m}^{0}\right) \quad \forall k, m \in \mathcal{T} \\
& s_{1_{k}} \in\{0,1\} \quad k \in \mathcal{G} \\
& s_{2_{k m}} \in\{0,1\} \quad k, m \in \mathcal{T}
\end{aligned}
$$

onde $P_{\text {perdas }}$ representa as perdas ativas na transmissão (2.4) e $N$ representa o número de variáveis de controles atuantes no sistema (2.11).

O modelo multiobjetivo com variáveis de controles contínuas e discretas do problema de FPO é definido como: 
$\min \quad\left[P_{\text {perdas }}, N\right]^{T}$

s.a: $\quad P_{k}-\sum_{m \in \mathcal{V}_{k}} P_{k m}(V, \theta, t)=0$

$\forall k \in \mathcal{G}^{\prime} \cup \mathcal{C}$

$Q_{k}+Q_{k}^{s h}\left(V_{k}, b_{k}^{s h}\right)-\sum_{m \in \mathcal{V}_{k}} Q_{k m}(V, \theta, t)=0 \quad \forall k \in \mathcal{C}$

$Q_{G_{k}}^{\min } \leq Q_{G_{k}}\left(V, \theta, t, b_{k}^{s h}\right) \leq Q_{G_{k}}^{\max }$

$\forall k \in \mathcal{G}$

$V_{k}^{\min } \leq V_{k} \leq V_{k}^{\max }$

$\forall k \in \mathcal{B}$

$s_{1_{k}}\left(V_{k}^{\text {min }}-V_{k}^{0}\right) \leq\left(V_{k}-V_{k}^{0}\right) \leq s_{1_{k}}\left(V_{k}^{\max }-V_{k}^{0}\right)$

$\forall k \in \mathcal{G}$

$s_{2_{k m}}\left(t_{k m}^{m i n}-t_{k m}^{0}\right) \leq\left(t_{k m}-t_{k m}^{0}\right) \leq s_{2_{k m}}\left(t_{k m}^{\max }-t_{k m}^{0}\right)$

$\forall k, m \in \mathcal{T}$

$s_{3_{k}}\left(b_{k}^{s h^{\text {min }}}-b_{k}^{s h^{0}}\right) \leq\left(b_{k}^{s h}-b_{k}^{s h^{0}}\right) \leq s_{3_{k}}\left(b_{k}^{s h^{\max }}-b_{k}^{s h^{0}}\right) \quad \forall k \in \mathcal{B}^{s h}$

$t_{k m} \in \mathcal{D}_{k m}^{t a p}$

$\forall k, m \in \mathcal{T}$

$b_{k}^{s h} \in \mathcal{D}_{k}^{s h}$

$\forall k \in \mathcal{B}^{s h}$

$s_{1_{k}} \in\{0,1\}$

$k \in \mathcal{G}$

$s_{2_{k m}} \in\{0,1\}$

$k, m \in \mathcal{T}$

$s_{3_{k}} \in\{0,1\}$

$k \in \mathcal{B}^{s h}$

onde $P_{\text {perdas }}$ representa as perdas ativas na transmissão (2.4) e $N$ representa o número de variáveis de controles atuantes no sistema (2.13).

Observe que ambos modelos (2.14) e (2.15), possuem variáveis contínuas e binárias, porém somente o modelo (2.15) contém variáveis discretas correspondente aos taps de transformadores e aos shunts de barras.

No próximo capítulo apresentam-se as estratégias para o tratamento de variáveis discretas e binárias necessárias para a resolução do problema de FPO com variáveis discretas e para o problema de FPO multiobjetivo com variáveis binárias. 


\section{Capítulo 3}

\section{Estratégias para o tratamento de variáveis discretas e binárias}

Neste capítulo são apresentadas as estratégias para a resolução de problemas de PNLCD e PNLIM. Três estratégias são utilizadas para o tratamento das variáveis discretas; a função de discretização senoidal; a função de discretização polinomial; e a combinação das funções de discretização senoidal e polinomial. Para o tratamento de variáveis binárias, duas abordagens são utilizadas; a função sigmoidal; e a função quadrática.

\subsection{Função de discretização Senoidal}

Nesta seção é apresentada a abordagem desenvolvida por Soler, Sousa e Costa (2012) e Soler, Asada e Costa (2013) para resolução de problemas de PNLCD. As variáveis discretas serão tratadas como contínuas por funções senoidais incorporadas a função objetivo do problema, transformando o problema de PNLCD em um problema de PNL.

Considere o seguinte problema de PNLCD:

$$
\begin{array}{lll}
\min & f(x) & \\
\text { s.a: } & g_{i}(x)=0 & i=1, \cdots, p \\
& h_{i}(x) \leq 0 & i=1, \cdots, q \\
& x_{1_{i}}^{\text {min }} \leq x_{1_{i}} \leq x_{1_{i}}^{\max } & i=1, \cdots, m_{1} \\
& x_{2_{i}} \in \mathcal{D}_{x_{2_{i}}} & i=1, \cdots, m_{2}
\end{array}
$$

onde $x_{1} \in \mathbb{R}^{m_{1}}$ é o vetor das variáveis contínuas e $x_{2} \in \mathcal{D}_{x_{2}} \subset \mathbb{R}^{m_{2}}$ é o vetor das variáveis discretas, com $x=\left(x_{1}, x_{2}\right)$ e $x \in \mathbb{R}^{n} ; x_{1}^{\min }, x_{1}^{\max } \in \mathbb{R}^{m_{1}}$ são os vetores dos limites inferior $\mathrm{e}$ superior das variáveis contínuas $x_{1} ; \mathcal{D}_{x_{2_{i}}}$ é o conjunto dos valores discretos de cada variável $x_{2_{i}}$; $f: \mathbb{R}^{n} \rightarrow \mathbb{R} ; g: \mathbb{R}^{n} \rightarrow \mathbb{R}^{p}$, com $p<n ;$ e $h: \mathbb{R}^{n} \rightarrow \mathbb{R}^{q}$. 
De acordo com Soler, Asada e Costa (2013), a função $\phi: \mathbb{R} \rightarrow \mathbb{R}$ para o tratamento de variáveis discretas $x_{2}$ na resolução de problemas como (3.1) é definida como:

$$
\phi\left(y_{i}\right)=\left[\operatorname{sen}\left(\frac{y_{i}}{y_{i}^{\text {sup }}-y_{i}^{\text {inf }}} \pi+\alpha_{i}\right)\right]^{2 \beta},
$$

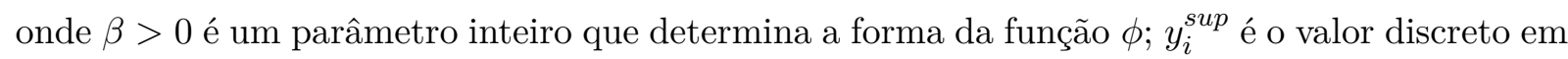
$\mathcal{D}_{x_{2_{i}}}$ imediatamente superior a $y_{i} ; y_{i}^{\text {inf }}$ é o valor discreto em $\mathcal{D}_{x_{2_{i}}}$ imediatamente inferior a $y_{i}$; e $\alpha_{i}$ é uma constante definida no intervalo $[0, \pi]$ tal que a função $\phi$ se anule quando $y_{i}=x_{2_{i}} \in \mathcal{D}_{x_{2_{i}}}$.

A função $\phi$ assume valores da seguinte forma:

$$
\phi\left(y_{i}\right)= \begin{cases}0 & \text { se } y_{i} \in \mathcal{D}_{x_{i}} \\ \rho>0 & \text { c.c. }\end{cases}
$$

ou seja, $\phi$ é nula se, e somente se, $y_{i}$ assumir valores discretos; e $\rho$ é um número real maior que zero.

Através da incorporação de funções $\phi$, para cada variável discreta, na função objetivo do problema (3.1), obtém-se o seguinte problema modificado:

$$
\begin{array}{lll}
\min & f(x)+\gamma \sum_{i=1}^{m_{2}} \phi\left(x_{2_{i}}\right) & \\
\text { s.a: } & g_{i}(x)=0 & i=1, \cdots, p \\
& h_{i}(x) \leq 0 & i=1, \cdots, q \\
x_{1_{i}}^{\text {min }} \leq x_{1_{i}} \leq x_{1_{i}}^{\max } & i=1, \cdots, m_{1} \\
x_{2_{i}}^{\text {min }} \leq x_{2_{i}} \leq x_{2_{i}}^{\max } & i=1, \cdots, m_{2}
\end{array}
$$

onde $\gamma>0$ é um parâmetro de penalidade que controla da amplitude da função $\phi ; x_{1} \in \mathbb{R}^{m_{1}}$ e $x_{2} \in \mathbb{R}^{m_{2}}$ são vetores de variáveis contínuas, com $x=\left(x_{1}, x_{2}\right)$ e $x \in \mathbb{R}^{n} ; f: \mathbb{R}^{n} \rightarrow \mathbb{R}$; $g: \mathbb{R}^{n} \rightarrow \mathbb{R}^{p}$, com $p<n$; e $h: \mathbb{R}^{n} \rightarrow \mathbb{R}^{q}$. No problema modificado (3.4), as funções $f, g$ e $h$ são de classe $C^{2}$ e $x_{2}^{\min }, x_{2}^{\max } \in \mathbb{R}^{m_{2}}$ são os vetores dos limites inferior e superior das variáveis contínuas $x_{2}, \operatorname{com} x_{2_{i}}^{\min }=\min \left\{\mathcal{D}_{x_{2_{i}}}\right\}$ e $x_{2_{i}}^{\max }=\max \left\{\mathcal{D}_{x_{2_{i}}}\right\}$ para $i=1, \cdots, m_{2}$.

A equação (3.4a) é denominada função objetivo aumentada, pois consiste na função objetivo do problema original acrescida das funções $\phi$.

\subsubsection{Definição da Constante $\alpha$}

Como, a função senoidal sen(y) é nula, somente quando $y=n \pi$, sendo $n \in \mathbb{Z}$, a determinação da constante $\alpha$ na função $\phi$ definida em função de $x_{2} \in \mathcal{D}_{x_{2}}$ é da seguinte forma:

$$
\frac{x_{2}}{x_{2}^{\text {sup }}-x_{2}^{\text {inf }}} \pi+\alpha=n \pi \quad \Rightarrow \quad \alpha=\left(n-\frac{x_{2}}{x_{2}^{\text {sup }}-x_{2}^{\text {inf }}}\right) \pi .
$$


Portanto, como $\alpha \in[0, \pi], n$ deve ser igual ao inteiro superior mais próximo de:

$$
\frac{x_{2}}{x_{2}^{\text {sup }}-x_{2}^{\text {inf }}} .
$$

Quando o conjunto de valores discretos $\mathcal{D}_{x_{2}}$ é formado por elementos igualmente espaçados, então $\alpha$ é igual para todos os intervalos de $\mathcal{D}_{x_{2}}$. No caso em que o conjunto de valores discretos $\mathcal{D}_{x_{2}}$ é formado por elementos não igualmente espaçados, então $\alpha$ deve ser especificado em função de cada intervalo de $\mathcal{D}_{x_{2}}$. Nestes casos, a função $\phi$ passa a ser definida por partes.

\subsubsection{Influência do Parâmetro $\beta$ na Função $\phi$}

Considere a seguinte variável $x \in \mathcal{D}_{x}=\{1,2,3,4\}$ para análise da influência do parâmetro $\beta$ na função $\phi$. Como os elementos do conjunto $\mathcal{D}_{x}$ são números inteiros, com espaçamento constante e igual a 1 , então $\alpha=0$ pois, dessa forma, a função $\phi$ se anula para todo $x \in \mathcal{D}_{x}$. A função $\phi$ definida em função de $\mathcal{D}_{x}$ é dada por (3.7) e está representada na Figura 3.1.

$$
\phi(x)=[\operatorname{sen}(x \pi)]^{2 \beta}
$$

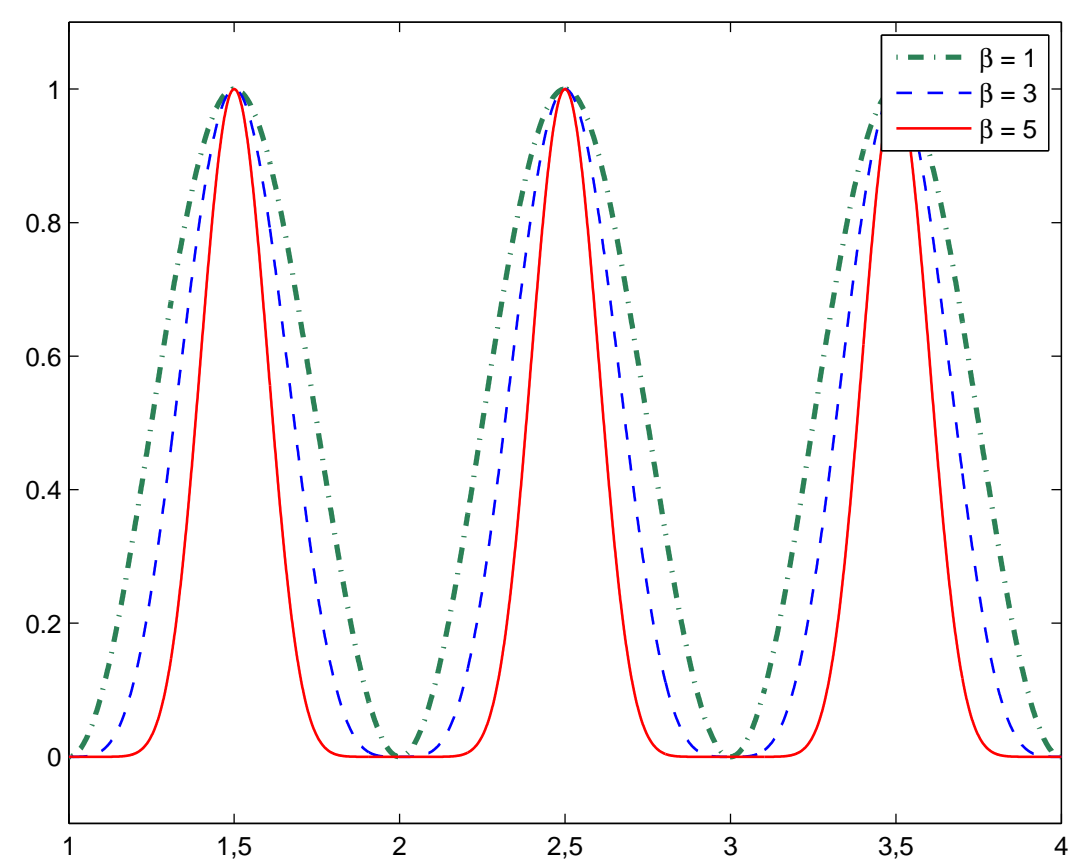

Figura 3.1 - Representação gráfica da função (3.7).

Note que o valor do parâmetro $\beta$ influência na forma da função $\phi$ : quanto maior for o valor dessa constante, mais "achatadas" e, portanto, menos penalizadas serão as regiões vizinhas aos valores definidos em $\mathcal{D}_{x}$. 


\subsubsection{Influência do parâmetro de penalidade $\gamma$ na Função $\phi$}

O parâmetro de penalidade $\gamma$ interfere na amplitude da função $\phi$, a Figura 3.2 apresenta esse efeito, considerando o exemplo (3.7) $\operatorname{com} \beta=1$.

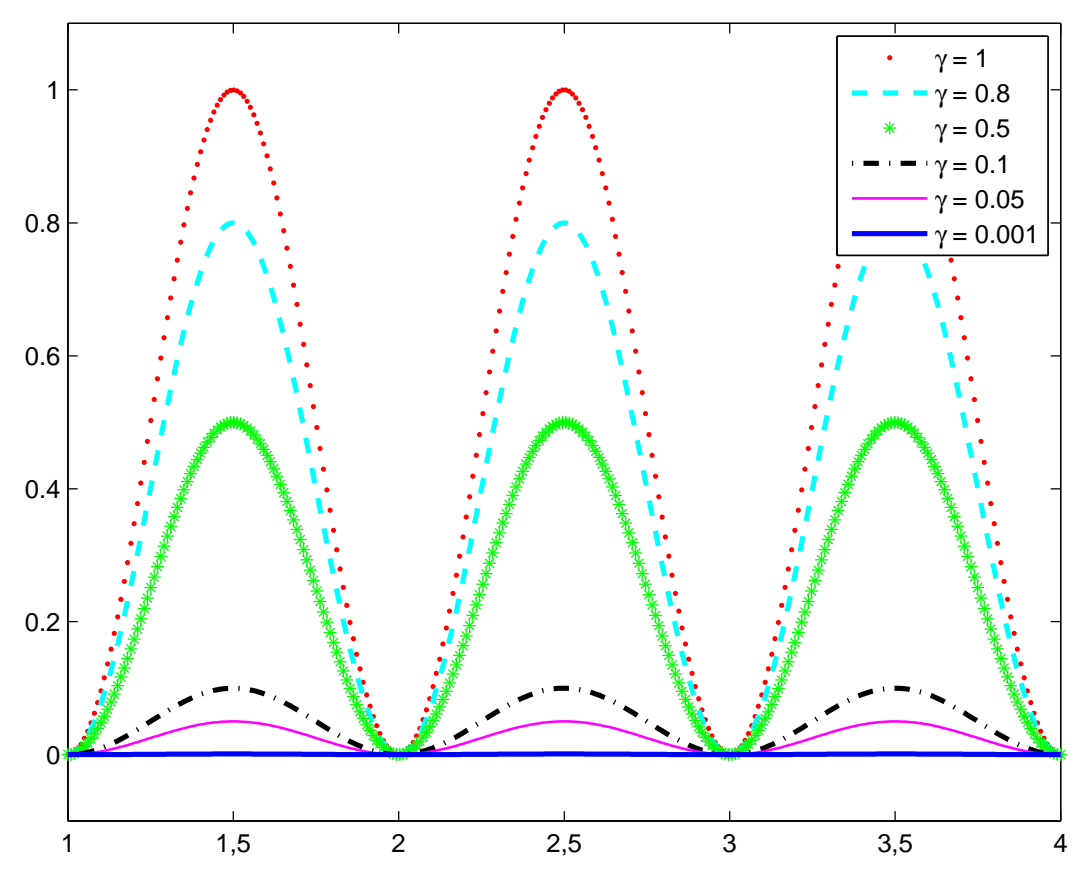

Figura 3.2 - Efeito do parâmetro de penalidade $\gamma$ na função $\phi$.

\subsubsection{Algoritmo de Discretização Utilizando a Função Senoidal}

O algoritmo consiste na resolução de uma sequência de problemas modificados (3.4) até que todas as variáveis associadas às variáveis discretas do problema original assumam valores discretos.

A determinação de um valor inicial para o parâmetro $\gamma$ é fundamental para que a solução do problema (3.4) seja encontrada. A fim de que o valor de $\gamma$ seja determinado corretamente, propõe-se a resolução de uma série de problemas como (3.4) para diferentes valores de $\gamma$, com $\gamma$ aumentando gradualmente. O ajuste do crescimento de $\gamma$ é dado por:

$$
\gamma^{k+1}=c \gamma^{k}
$$

onde $c$ é o fator de ajuste do crescimento de $\gamma$, definido no intervalo $(1,10]$, e $k$ representa a $k$-ésima iteração do algoritmo.

O processo de resoluções sucessivas do problema (3.4) e de ajuste do parâmetro $\gamma$ continua até que um dos seguintes critérios sejam satisfeitos. 
1. Critério de convergência: avalia a discretização das variáveis $x_{2}$ na solução corrente. Se a maior diferença entre uma variável $x_{2}^{k}$ e seu valor discreto mais próximo for menor que uma tolerância predeterminada, isto significa que a discretização definida por $x_{2}^{k}$ é suficientemente boa e o algoritmo é interrompido. Matematicamente, este critério pode ser representado por:

$$
\left\|x_{2_{i}}^{k}-x_{2_{i}}^{\prime}\right\|_{\infty} \leq \xi_{1}
$$

onde $x_{2_{i}}^{\prime} \in \mathcal{D}_{x_{2_{i}}}$ é o valor discreto mais próximo de $x_{2_{i}}^{k}$, para $i=1, \cdots, m_{2}$, e $\xi_{1}$ é a tolerância de discretização do algoritmo.

2. Critério de parada: avalia os ajustes nas variáveis $x_{2}$ entre duas iterações consecutivas. Se o maior ajuste em uma variável $x_{2}$ for menor que uma tolerância predeterminada, o algoritmo é interrompido. Nesse caso, a solução do problema (3.4) pode não corresponder a uma solução do problema (3.1), e este critério é utilizado para encerrar o algoritmo em caso de falha. Matematicamente, esse critério pode ser representado por:

$$
\left\|x_{2_{i}}^{k}-x_{2_{i}}^{k-1}\right\|_{\infty}<\xi_{2}
$$

onde $\xi_{2}$ é a tolerância de ajuste das variáveis discretas entre duas iterações consecutivas.

Portanto, a resolução de um problema de PNLCD como (3.1) é dada pelo Algoritmo 1.

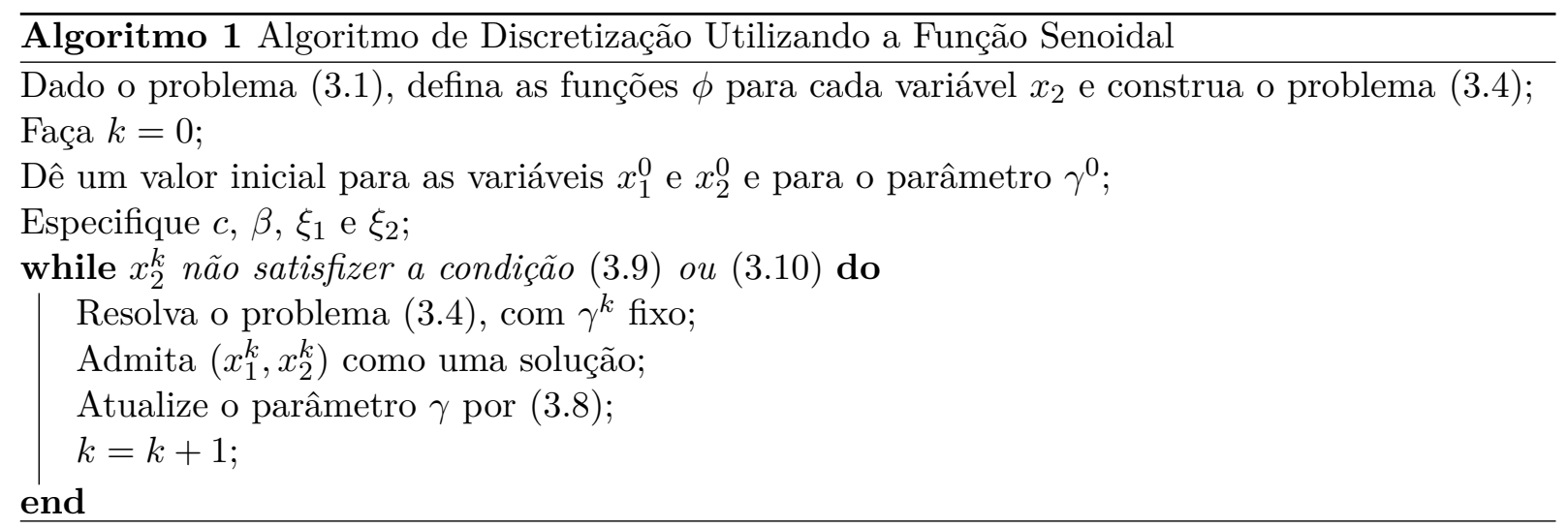




\subsection{Função de discretização Polinomial}

Baseado em Gisvold e Moe (1972), a função de discretização polinomial $\psi: \mathbb{R} \rightarrow \mathbb{R}$ para o tratamento das variáveis discretas $x_{2}$ na resolução de problemas como (3.1), assume valores da seguinte forma:

$$
\psi\left(y_{i}\right)= \begin{cases}0 & \text { se } y_{i} \in \mathcal{D}_{x_{2_{i}}} \\ \mu>0 & \text { c.c. }\end{cases}
$$

onde $\psi\left(y_{i}\right)$ é dada por:

$$
\psi\left(y_{i}\right)=\left(4 q_{i}\left(1-q_{i}\right)\right)^{2 \beta_{1}}
$$

e

$$
q_{i}=\frac{\left(y_{i}-y_{i}^{i n f}\right)}{\left(y_{i}^{\text {sup }}-y_{i}^{i n f}\right)}
$$

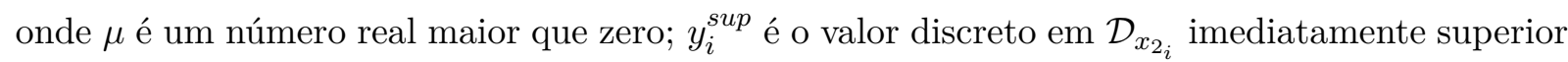
a $y_{i}$ e $y_{i}^{\text {inf }}$ é o valor discreto em $\mathcal{D}_{x_{2_{i}}}$ imediatamente inferior a $y_{i}$. Esta função é normalizada e simétrica. O valor máximo de cada termo da soma (3.12) é de 1.0, e para qualquer $\beta_{1}>1$, a função é contínua na sua primeira derivada em relação ao ponto de diferenciação.

Incorporando as funções $\psi$ na função objetivo do problema (3.1), obtém-se o seguinte problema modificado:

$$
\begin{aligned}
& \min \quad f(x)+\nu \sum_{i=1}^{m_{2}} \psi\left(x_{2_{i}}\right) \\
& \text { s.a: } \quad g_{i}(x)=0 \quad i=1, \cdots, p \\
& h_{i}(x) \leq 0 \quad i=1, \cdots, q \\
& x_{1_{i}}^{\text {min }} \leq x_{1_{i}} \leq x_{1_{i}}^{\max } \quad i=1, \cdots, m_{1} \\
& x_{2_{i}}^{\min } \leq x_{2_{i}} \leq x_{2_{i}}^{\max } \quad i=1, \cdots, m_{2}
\end{aligned}
$$

onde $\nu$ é o parâmetro de penalidade que controla a amplitude da função $\psi$.

Note que a única diferença entre o problema modificado (3.14) e o problema modificado (3.4) é a função de discretização adotada. 


\subsubsection{Influência do Parâmetro $\beta_{1}$ na Função $\psi$}

A Figura 3.3 apresenta a função $\psi(y)$ para diferentes valores de $\beta_{1}$. O parâmetro $\beta_{1}$ influencia a forma da função $\psi$ : quanto maior for o valor desse parâmetro, maior será a quantidade de valores próximos de zero na vizinhança dos valores discretos. A Figura 3.4 mostra somente a curva da função $\psi(y)$ quando $\beta_{1}=10$, ficando bem claro o efeito de $\beta_{1}$ no formato da função $\psi$.

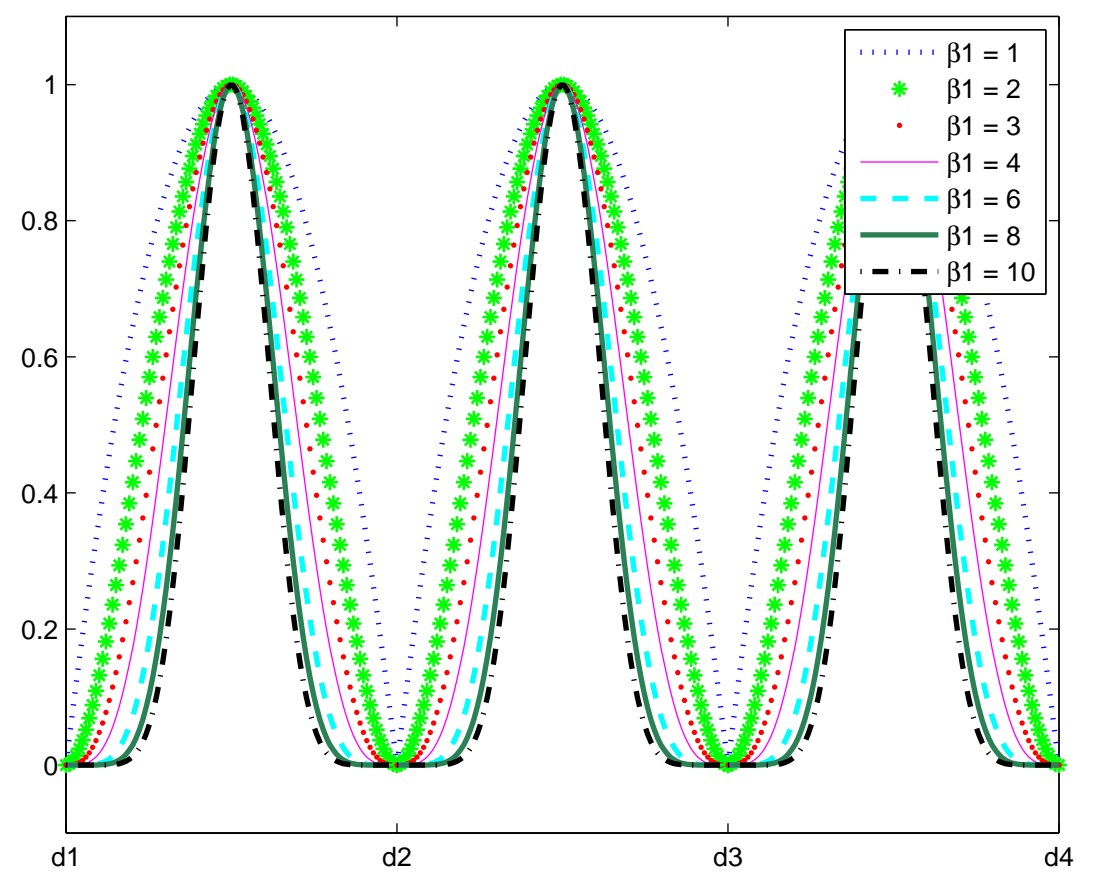

Figura 3.3 - Representação gráfica da função (3.12) com diferentes valores de $\beta_{1}$.

Vale ressaltar que a vizinhança de um valor discreto não assume valores nulos na função $\psi(y)$, entretanto, assume valores suficientemente pequenos para que, dependendo da tolerância de discretização assumida, possam ser considerados nulos. A Figura 3.5 é um "zoom" da Figura 3.4 na proximidade de um valor discreto, note que na região da circunferência traçada, a vizinhança de d2 aparenta ter a mesma imagem em zero, isso acontece pois os valores da vizinhança são quase nulos. 


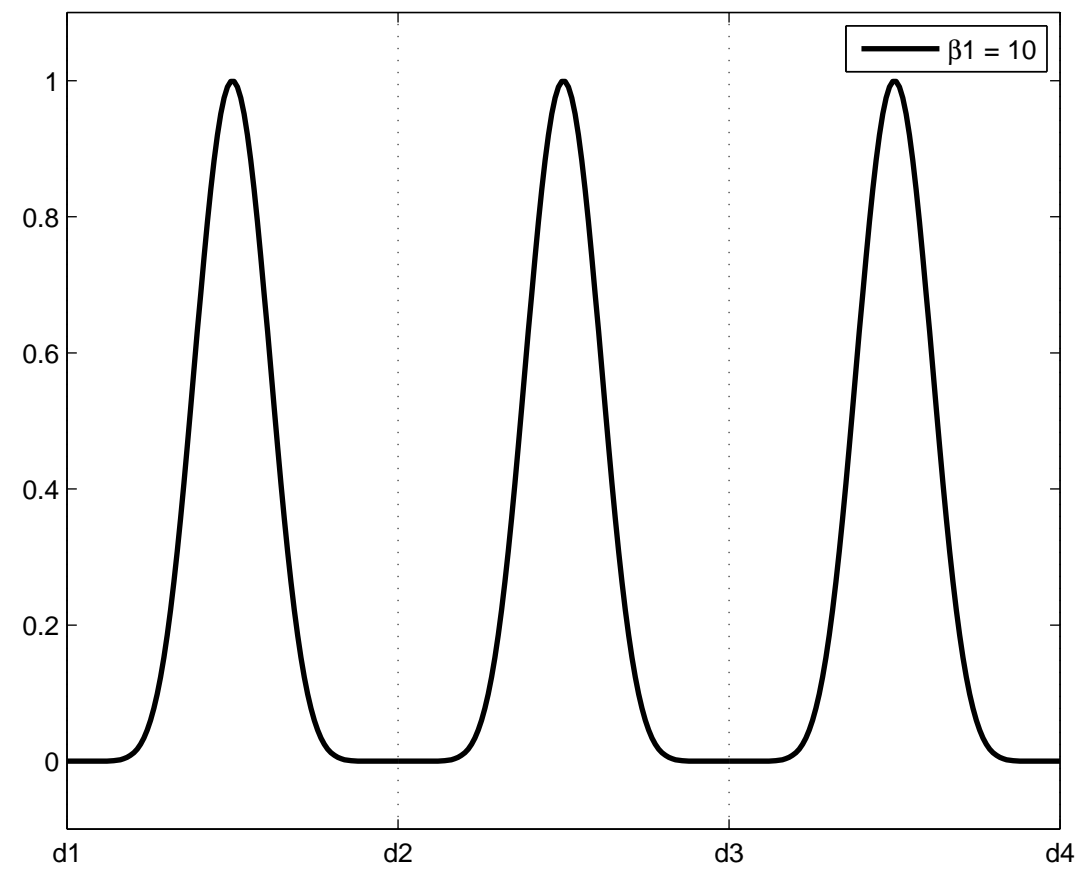

Figura 3.4 - Representação gráfica da função (3.12) com $\beta_{1}=10$.

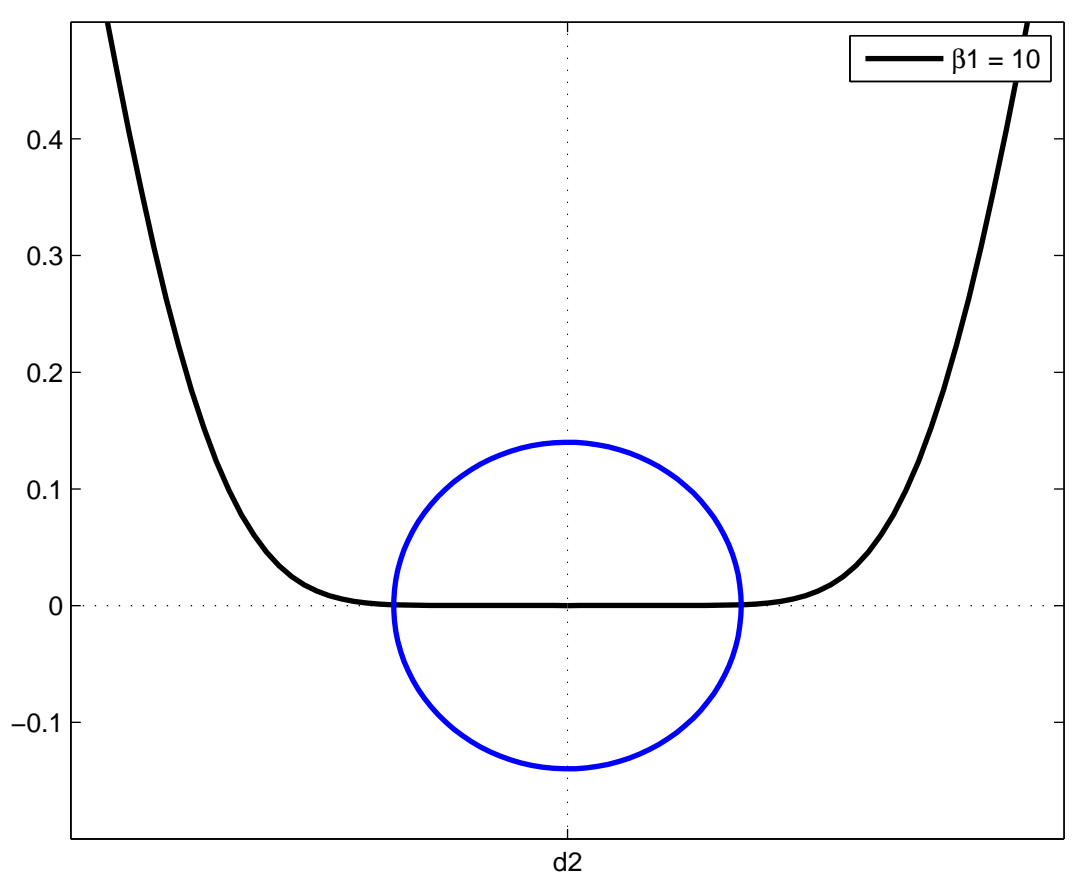

Figura 3.5 - Representação gráfica da função (3.12) com $\beta_{1}=10$ na proximidade do valor discreto d2. 


\subsubsection{Influência do parâmetro de penalidade $\nu$ na Função $\psi$}

O parâmetro de penalidade $\nu$ interfere na amplitude da função $\psi$, a Figura 3.6 apresenta esse efeito.

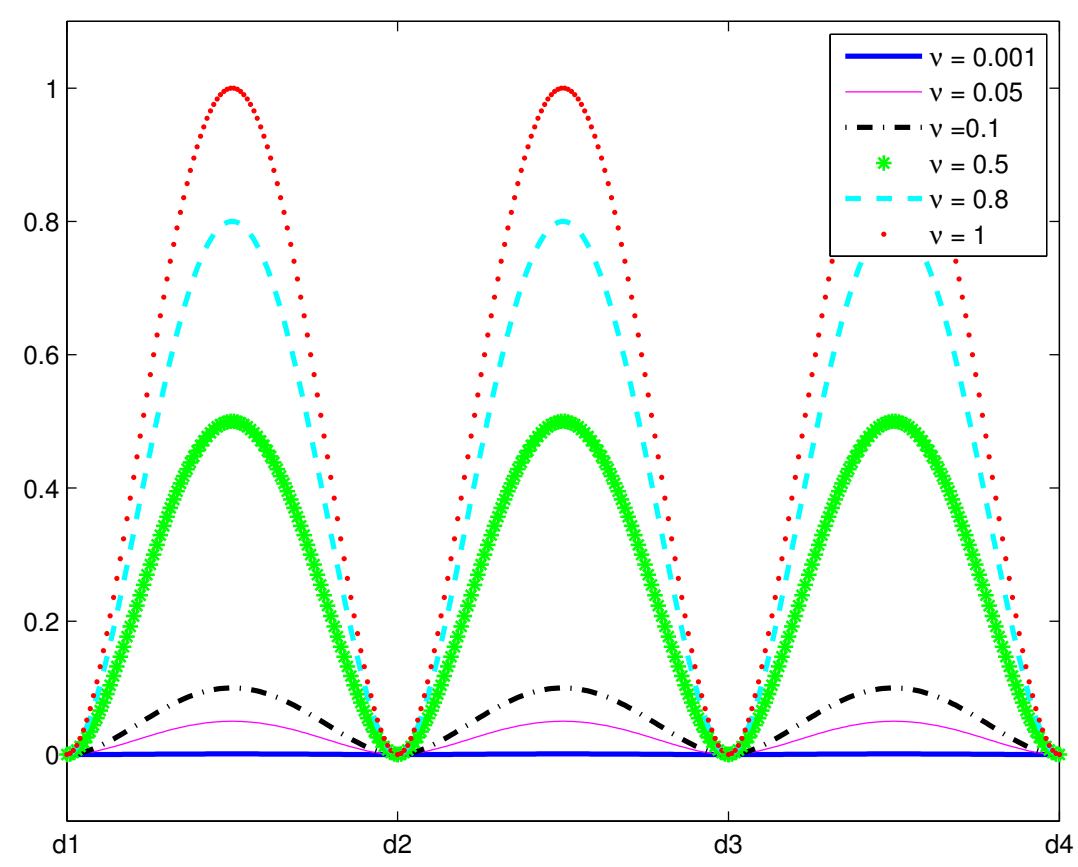

Figura 3.6 - Efeito do parâmetro de penalidade $\nu$ na função $\psi$.

Considere o seguinte exemplo:

$$
\begin{array}{ll}
\min & f(x)=x^{4}+x^{3}-0.1 x \\
\text { s.a: } & x \in \mathcal{D}
\end{array}
$$

onde $\mathcal{D}=\{-1,-0.75,-0.5,0,0.25,0.5\}$ é o conjunto dos valores discretos no qual $x$ pertence.

A Figura 3.7 exibe a representação gráfica do problema (3.15), onde as soluções factíveis são as intersecções entre a curva $f(x)$ e a restrição do problema.

O problema (3.15) é modificado pela função $\psi$ da seguinte forma:

$$
\begin{aligned}
& \min \quad x^{4}+x^{3}-0.1 x+\nu\left(4\left(\frac{\left(x-x^{\text {inf }}\right)}{\left(x^{\text {sup }}-x^{\text {inf }}\right)}\right) \cdot\left(1-\frac{\left(x-x^{\text {inf }}\right)}{\left(x^{\text {sup }}-x^{\text {inf }}\right)}\right)\right)^{\beta_{1}} \\
& \text { s.a: } \quad-1.0 \leq x \leq 0.5
\end{aligned}
$$




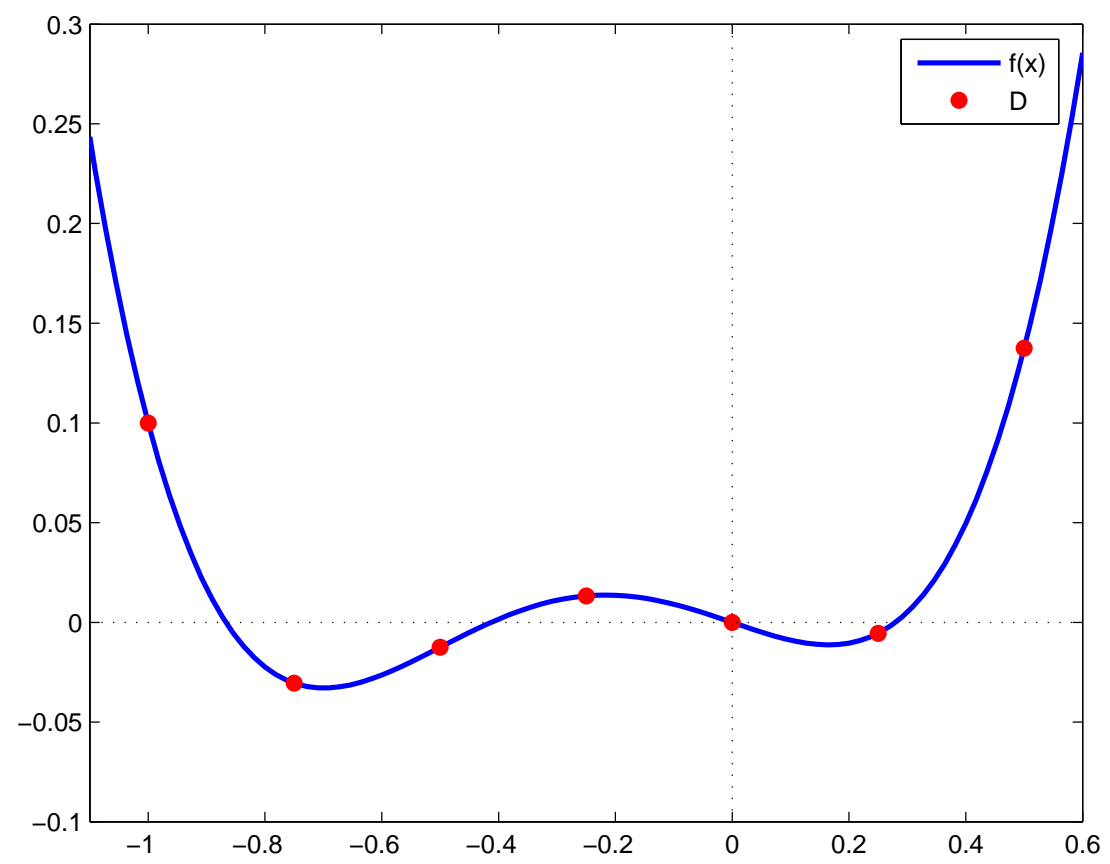

Figura 3.7 - Representação gráfica do problema (3.15), onde $x \in \mathcal{D}$.

As Figuras 3.8, 3.9 e 3.10 exibem a representação gráfica do problema (3.16) com diferentes valores de $\nu$. Como observado, $\nu$ influencia na amplitude da função $\psi$, o que pode ser interpretado como a semelhança entre a função objetivo do problema original e a função objetiva do problema aumentado: quanto menor o valor de $\nu$, maior a semelhança entre elas.

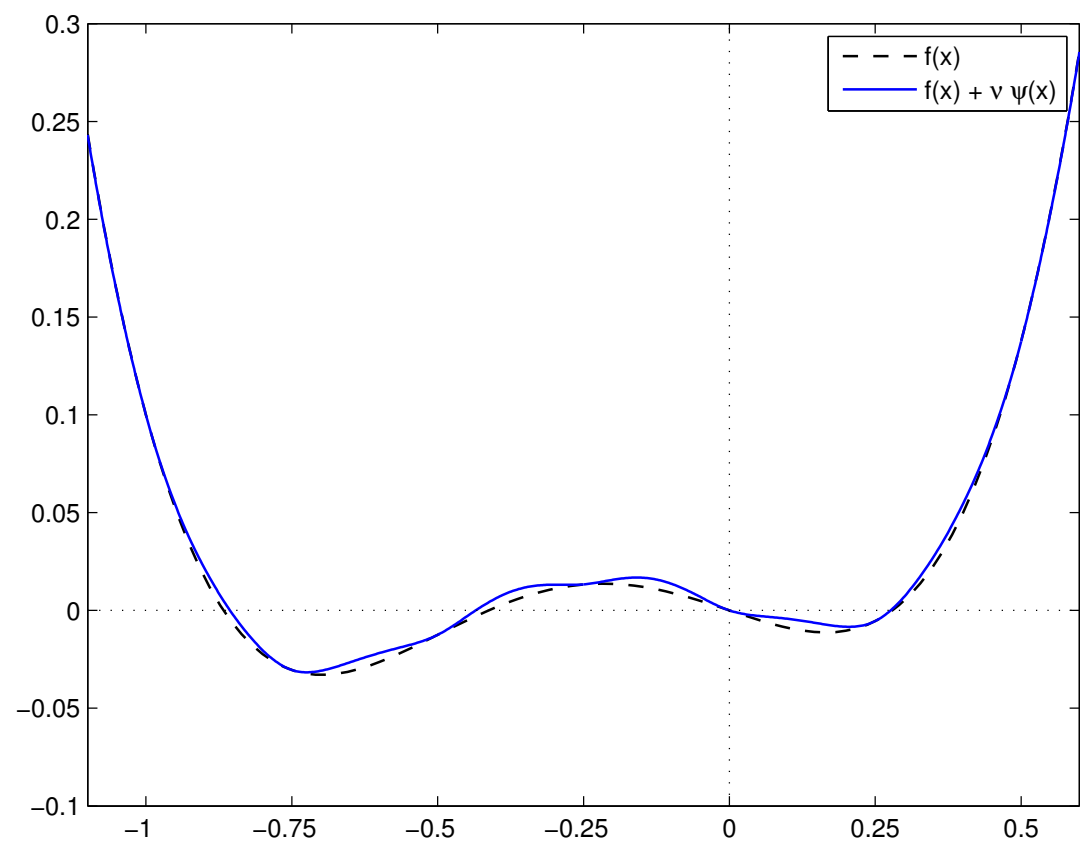

Figura 3.8 - Representação gráfica do problema (3.16), com $\beta_{1}=1$ e $\nu=0.005$. 


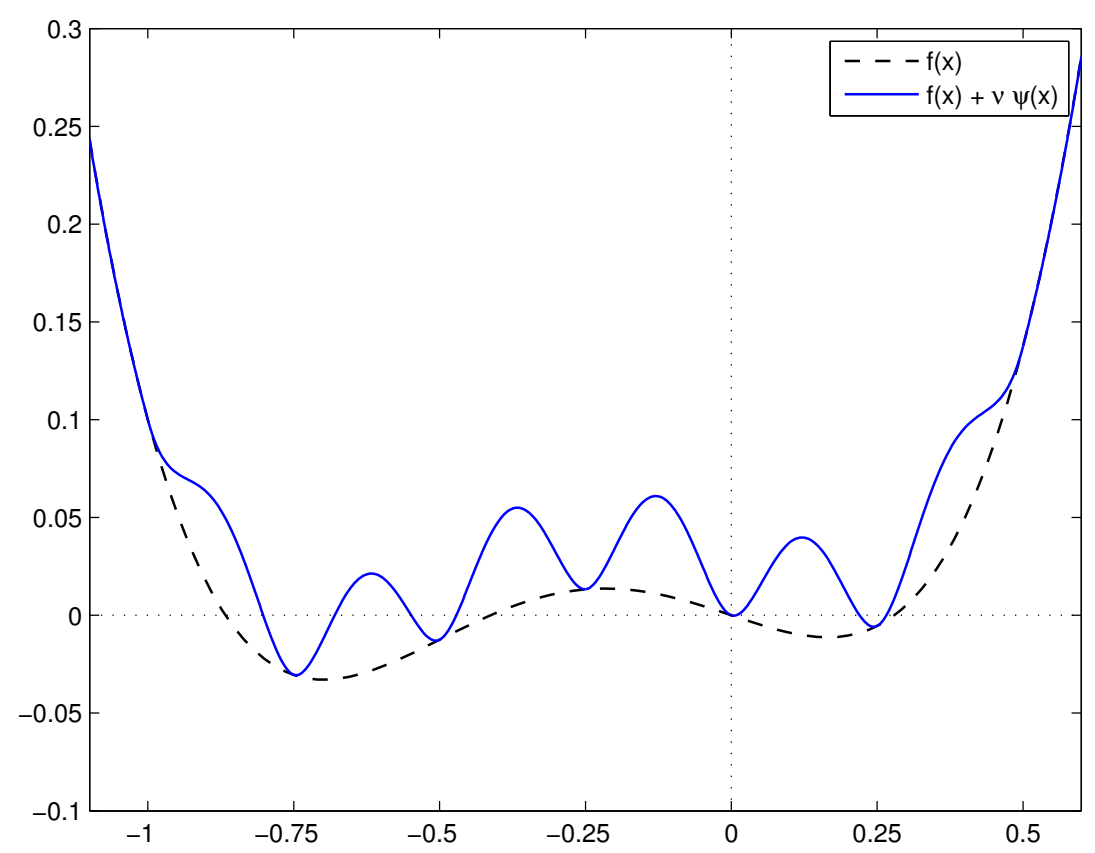

Figura 3.9 - Representação gráfica do problema (3.16), com $\beta_{1}=1$ e $\nu=0.05$.

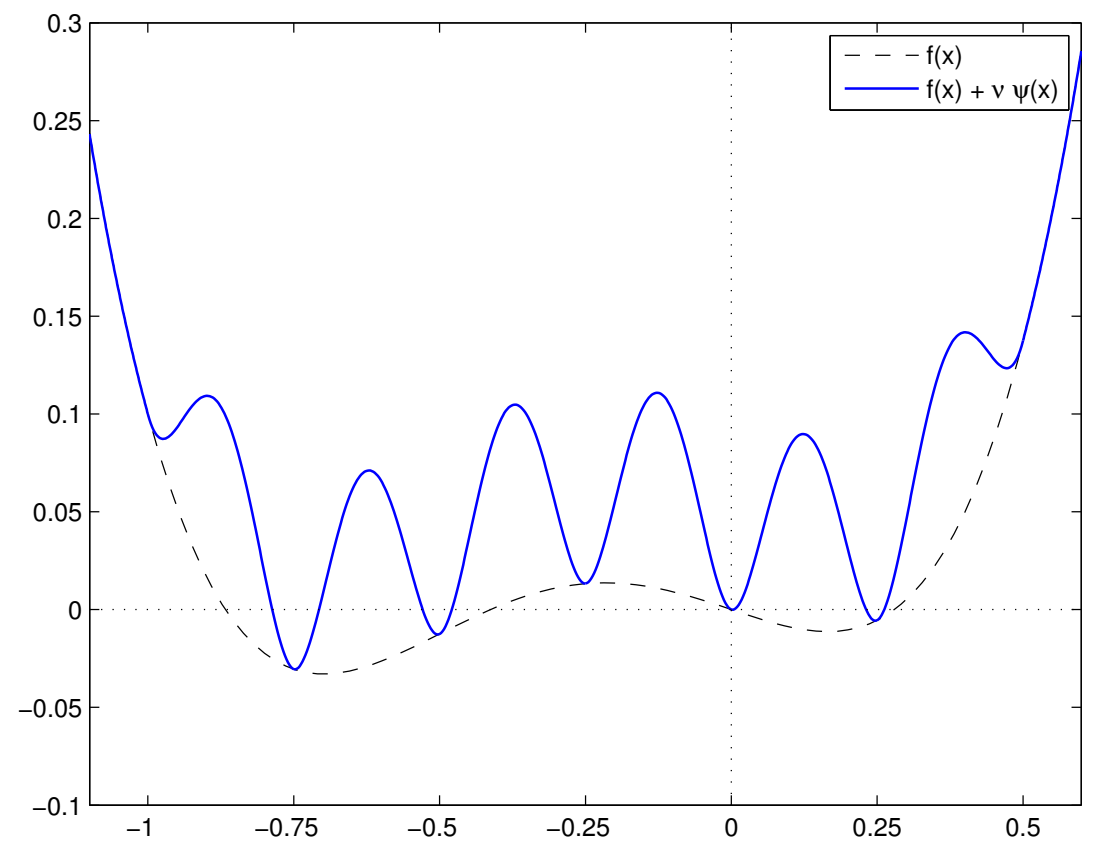

Figura 3.10 - Representação gráfica do problema (3.16), com $\beta_{1}=1$ e $\nu=0.1$.

É importante notar que a função $\psi$ pode tratar tanto variáveis discretas atualizadas por passos iguais, ou seja, o conjunto no qual variáveis discretas podem assumir valores é igualmente espaçado, como as do problema (3.15), quanto variáveis discretas atualizadas por passos diferentes. Ou seja, o conjunto no qual variáveis discretas podem assumir valores não é igualmente espaçado. Para ilustrar este segundo caso, considere o problema (3.15) agora com 
$\mathcal{D}_{1}=\{-1,-0.75,-0.5,0.5\}$ sendo o conjunto dos valores discretos que $x$ pode assumir valores. As Figuras 3.11, 3.12 e 3.13 apresentam este novo problema.

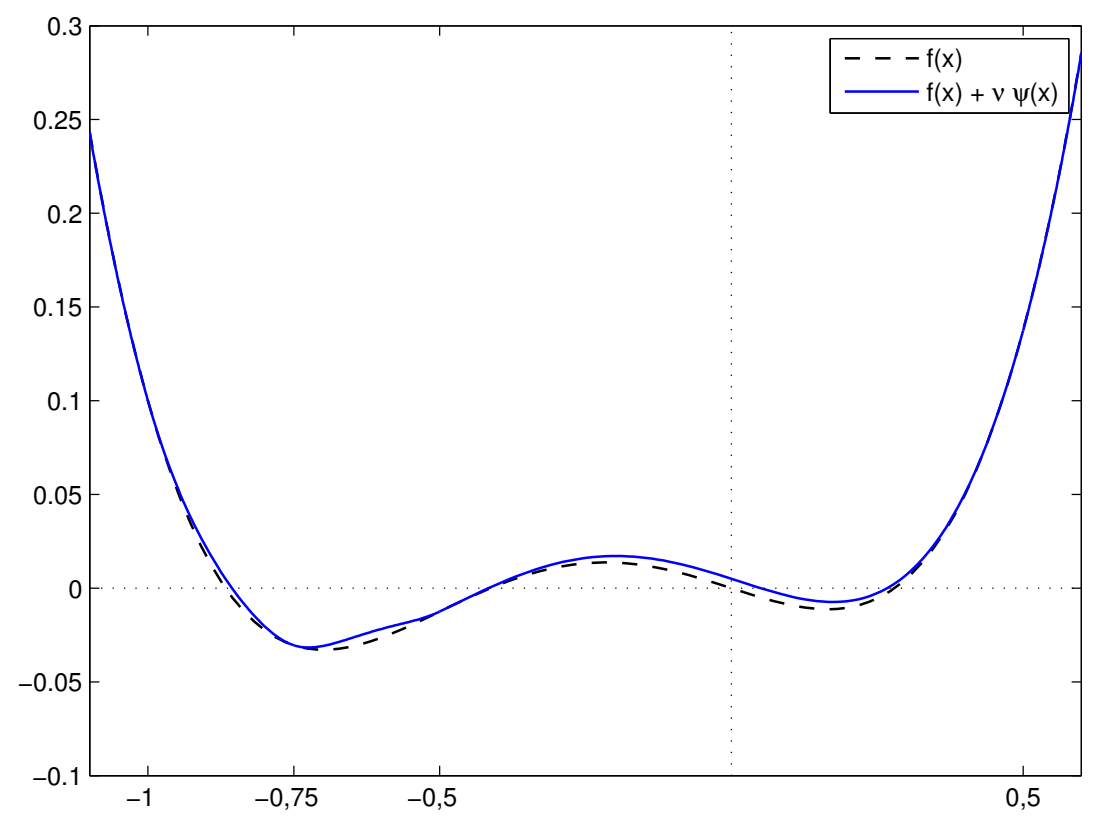

Figura 3.11 - Representação gráfica do problema (3.16),com $\mathcal{D}_{1}=\{-1,-0.75,-0.5,0.5\}, \beta_{1}=1$ e $\nu=0.005$.

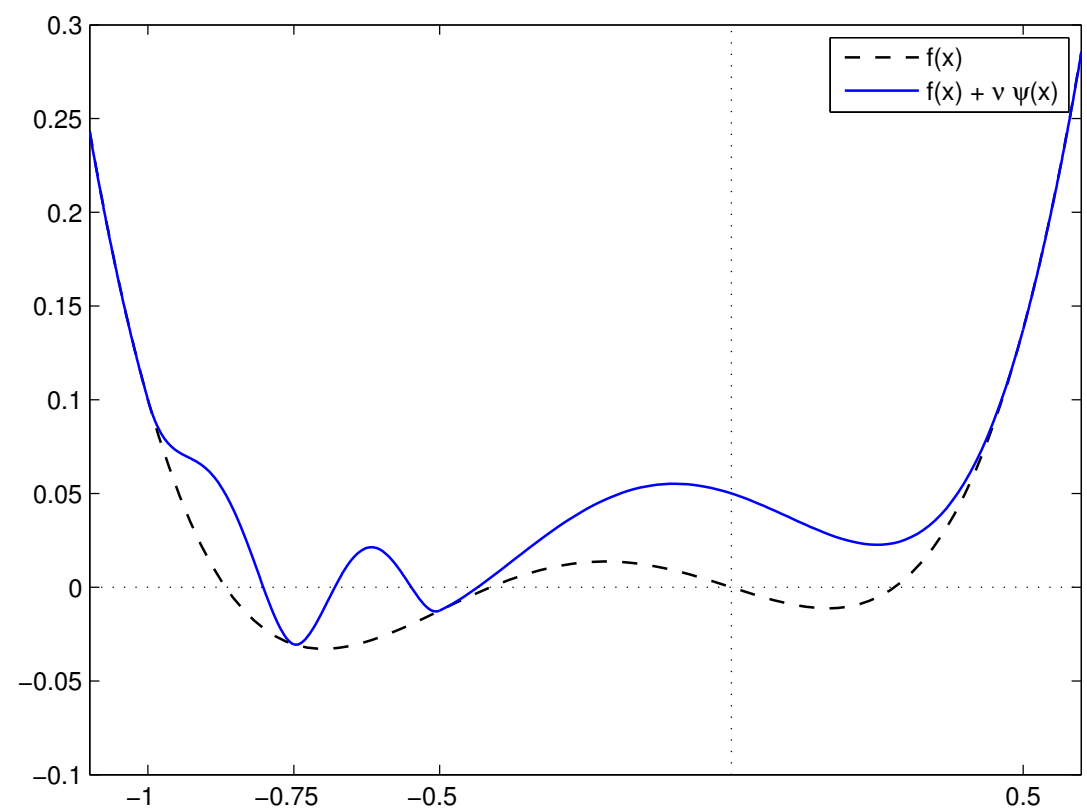

Figura 3.12 - Representação gráfica do problema (3.16), com $\mathcal{D}_{1}=\{-1,-0.75,-0.5,0.5\}, \beta_{1}=1$ e $\nu=0.05$. 


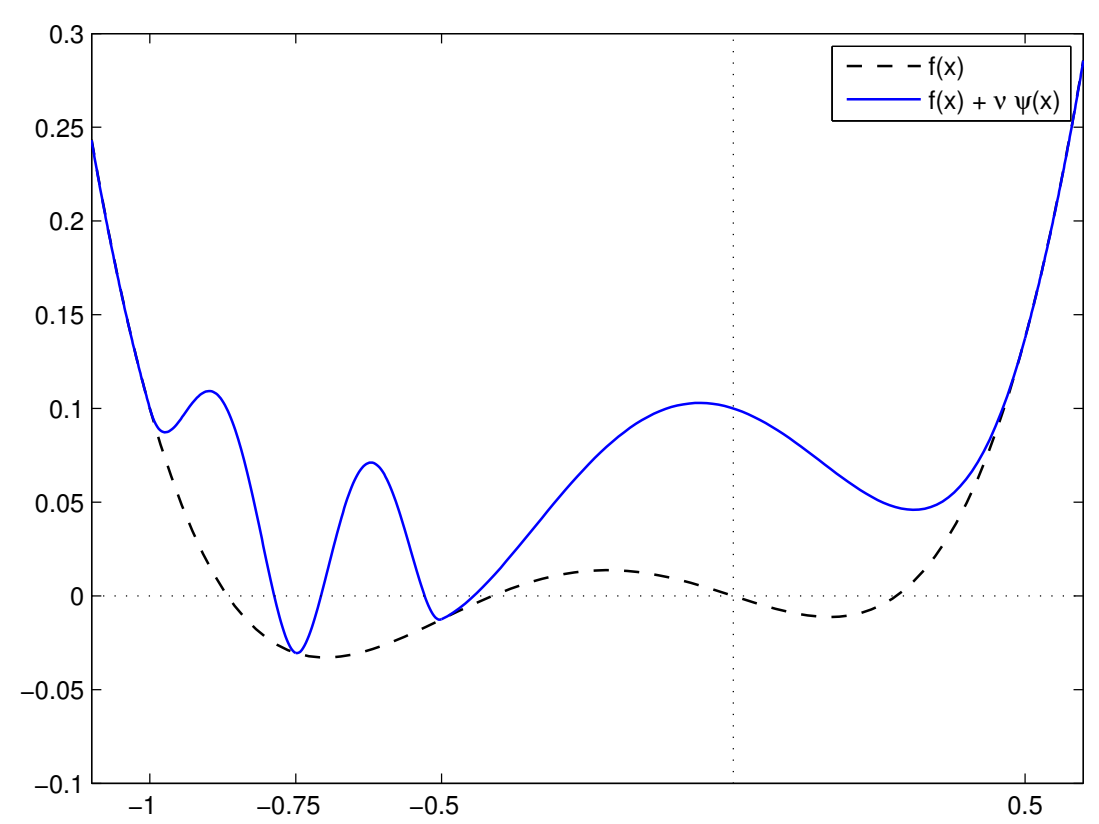

Figura 3.13 - Representação gráfica do problema (3.16), com $\mathcal{D}_{1}=\{-1,-0.75,-0.5,0.5\}, \beta_{1}=1$ e $\nu=0.1$.

\subsubsection{Estimativa inicial para $\nu$}

Considere o problema modificado (3.14). Inicialmente, o parâmetro de penalidade (fator de ponderação), $\nu_{k}$, deve ser suficientemente pequeno para se obter uma superfície de resposta unimodal. Isto pode ser alcançado definindo:

$$
\nu \psi^{\prime} \ll F^{\prime}
$$

onde

$$
\psi^{\prime}=4^{\bar{\beta}} \bar{\beta} \frac{(\bar{\beta}-1)^{(\bar{\beta}-1)}}{2}(2 \bar{\beta}-1)^{\frac{1}{2}-\bar{\beta}}
$$

representa uma estimativa do gradiente máximo para a superfície $\psi, \bar{\beta}=2 \beta_{1}$ e $F^{\prime}$ é a medida do grau de inclinação da superfície de resposta $f$, onde

$$
F^{\prime}=\sqrt{\frac{\nabla f^{T} \nabla f}{n}}
$$

$\nabla f$ é o gradiente da função objetivo $f$ com relação as variáveis discretas e $n$ é o tamanho de $\nabla f$.

Com base na equação (3.17), verifica-se razoável determinar o valor inicial, $\nu_{0}$, pela seguinte fórmula: 


$$
\nu_{0}=\delta \cdot \frac{F^{\prime}\left(x^{0}\right)}{\psi^{\prime}\left(\beta_{1}\right)}
$$

com

$$
0.001 \leq \delta \leq 0.1
$$

Temos que, $x^{0}$ representa os valores iniciais das variáveis contínuas e discretas para o início da busca. Mais informações sobre a determinação de $\nu_{0}$ podem ser encontradas em Gisvold e Moe (1972).

\subsubsection{Algoritmo Proposto Utilizando a Função Polinomial}

Dado $\nu_{0}$, propõe-se a resolução de uma série de problemas como (3.14) com $\nu$ crescendo gradualmente. O ajuste do crescimento de $\nu$ é dado por:

$$
\nu^{k+1}=\ell \nu^{k}
$$

onde $\ell$ é o fator de ajuste do crescimento de $\nu$, definido no intervalo $(1,10]$, e $k$ representa a $k$-ésima iteração do algoritmo.

O processo de resoluções sucessivas do problema (3.14) e de ajuste do parâmetro $\nu$ continuam até que um dos critérios de parada, (3.9) ou (3.10), sejam satisfeitos.

Portanto, a resolução de um problema de PNLCD como (3.1) é dada pelo Algoritmo 2.

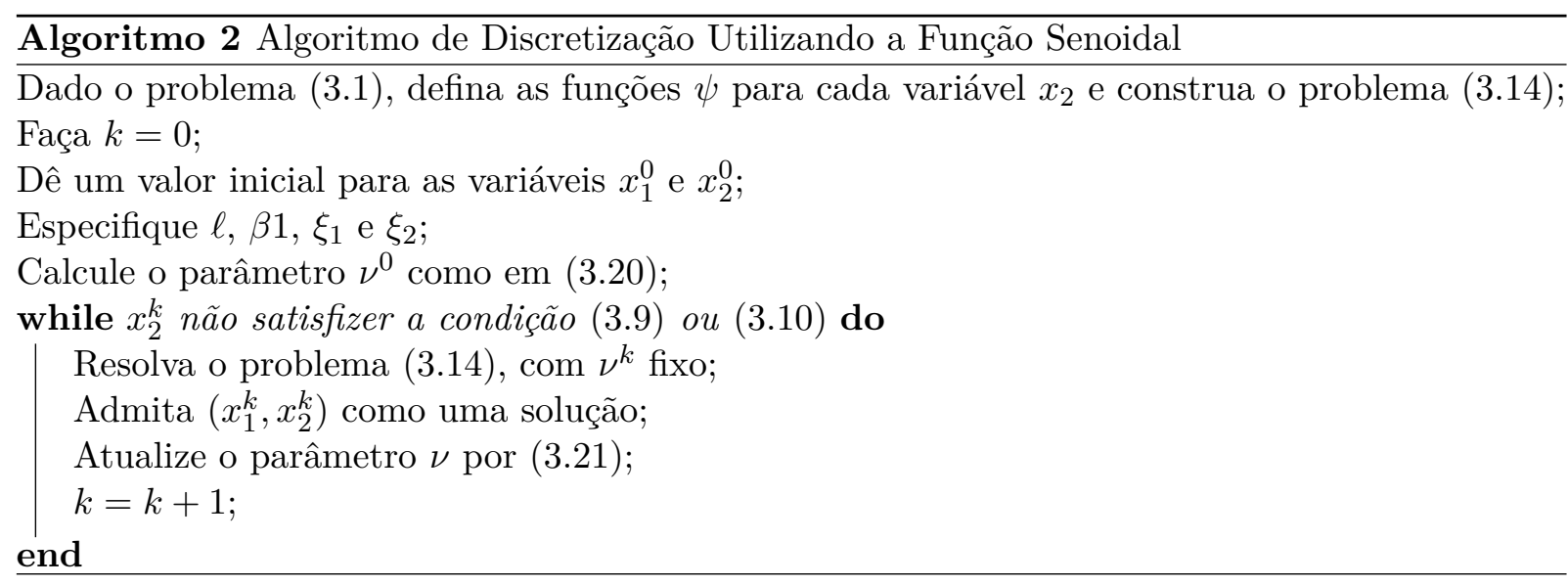

Encontrar uma solução ótima para este problema modificado (3.14) equivale encontrar uma solução factível para o problema original (3.1).

\subsection{Estratégia de Discretização Mista}

Esta estratégia visa abranger as melhores qualidades das funções de discretização $\phi$ e $\psi$ descritas nas seções 3.1 e 3.2, respectivamente. As variáveis discretas são tratadas pela combinação das funções de discretização senoidal e polinomial, que são contínuas, suaves e 
diferenciáveis em seus valores discretos. A função discretização senoidal é utilizada para lidar com variáveis discretas espaçadas regularmente devido à sua periodicidade. Por outro lado, a função polinomial de discretização é usada para lidar com as variáveis discretas espaçadas irregularmente.

Considere o seguinte problema de PNLCD:

$$
\begin{array}{lll}
\min & f(x) & \\
\text { s.a: } & g_{i}(x)=0 & i=1, \cdots, p \\
& h_{i}(x) \leq 0 & i=1, \cdots, q \\
& x_{1_{i}}^{\text {min }} \leq x_{1_{i}} \leq x_{1_{i}}^{\max } & i=1, \cdots, m_{1} \\
& x_{2_{i}} \in \mathcal{D}_{x_{2_{i}}} & i=1, \cdots, m_{2} \\
& x_{3_{i}} \in \mathcal{D}_{x_{3_{i}}} & i=1, \cdots, m_{3}
\end{array}
$$

onde $x_{1} \in \mathbb{R}^{m_{1}}$ representa as variáveis contínuas do problema; $x_{2} \in \mathcal{D}_{x_{2}} \subset \mathbb{R}^{m_{2}}$ representa as variáveis discretas ajustadas por passos discretos constantes; $x_{3} \in \mathcal{D}_{x_{3}} \subset \mathbb{R}^{m_{3}}$ representa as variáveis discretas ajustadas por passos discretos distintos; com $x=\left(x_{1}, x_{2}, x_{3}\right)$ e $x \in \mathbb{R}^{n}$; $f: \mathbb{R}^{n} \rightarrow \mathbb{R} ; g: \mathbb{R}^{n} \rightarrow \mathbb{R}^{p}$, com $p<n ;$ e $h: \mathbb{R}^{n} \rightarrow \mathbb{R}^{q} ; \mathcal{D}_{x_{2}}$ é o conjunto de variáveis discretas com espaçamento constante; $\mathcal{D}_{x_{3}}$ é o conjunto de variáveis discretas com espaçamento distintos; $x_{1}^{\text {min }}, x_{1}^{\max } \in \mathbb{R}^{m_{1}}$ são os vetores dos limites inferior e superior das variáveis contínuas $x_{1}$.

Incorporando a função $\phi(3.2)$ e a função $\psi(3.12)$ no problema (3.22) temos o seguinte problema modificado:

$$
\begin{aligned}
& \min f(x)+\gamma \sum_{i=1}^{m_{2}} \phi\left(x_{2_{i}}\right)+\nu \sum_{i=1}^{m_{3}} \psi\left(x_{3_{i}}\right) \\
& \text { s.a: } \quad g_{i}(x)=0 \\
& h_{i}(x) \leq 0 \\
& x_{1_{i}}^{\text {min }} \leq x_{1_{i}} \leq x_{1_{i}}^{\max } \\
& x_{2_{i}}^{\min } \leq x_{2_{i}} \leq x_{2_{i}}^{\max } \\
& x_{3_{i}}^{\min } \leq x_{3_{i}} \leq x_{3_{i}}^{\max } \\
& i=1, \cdots, p \\
& i=1, \cdots, q \\
& i=1, \cdots, m_{1} \\
& i=1, \cdots, m_{2} \\
& i=1, \cdots, m_{3}
\end{aligned}
$$

onde $\gamma>0$ é um parâmetro de penalidade que controla a amplitude da função $\phi ; \nu>0$ é um parâmetro de penalidade que controla a amplitude da função $\psi ; x_{1} \in \mathbb{R}^{m_{1}}, x_{2} \in \mathbb{R}^{m_{2}}$ e $x_{3} \in \mathbb{R}^{m_{3}}$ são vetores de variáveis contínuas, com $x=\left(x_{1}, x_{2}, x_{3}\right)$ e $x \in \mathbb{R}^{n} ; f: \mathbb{R}^{n} \rightarrow \mathbb{R} ; g: \mathbb{R}^{n} \rightarrow \mathbb{R}^{p}$, $\operatorname{com} p<n$; e $h: \mathbb{R}^{n} \rightarrow \mathbb{R}^{q}$. No problema modificado (3.23), as funções $f, g$ e $h$ são de classe $C^{2}$; $x_{2}^{\min }, x_{2}^{\max } \in \mathbb{R}^{m_{2}}$ são os vetores dos limites inferior e superior das variáveis contínuas $x_{2}$, com $x_{2_{i}}^{\min }=\min \left\{D_{x_{2_{i}}}\right\}$ e $x_{2_{i}}^{\max }=\max \left\{D_{x_{2_{i}}}\right\}$ para $i=1, \cdots, m_{2} ; x_{3}^{\min }, x_{3}^{\max } \in \mathbb{R}^{m_{3}}$ são os vetores dos limites inferior e superior das variáveis contínuas $x_{3}, \operatorname{com} x_{3_{i}}^{\min }=\min \left\{D_{x_{3_{i}}}\right\}$ e $x_{3_{i}}^{\max }=\max \left\{D_{x_{3_{i}}}\right\}$ para $i=1, \cdots, m_{3}$. 


\subsubsection{Algoritmo Proposto Utilizando a Estratégia de Discretização Mista}

As funções $\phi(3.2)$ e $\psi(3.12)$ e seus respectivos parâmetros são tratadas como descritas nas seções 3.1 e 3.2. A resolução de um problema de PNLCD como (3.23) é dada pelo Algoritmo 3. Novamente, quando a resolução sucessiva de problemas (3.23) pelo Algoritmo 3 encontra uma solução discreta, esta é uma solução factível para o problema (3.22).

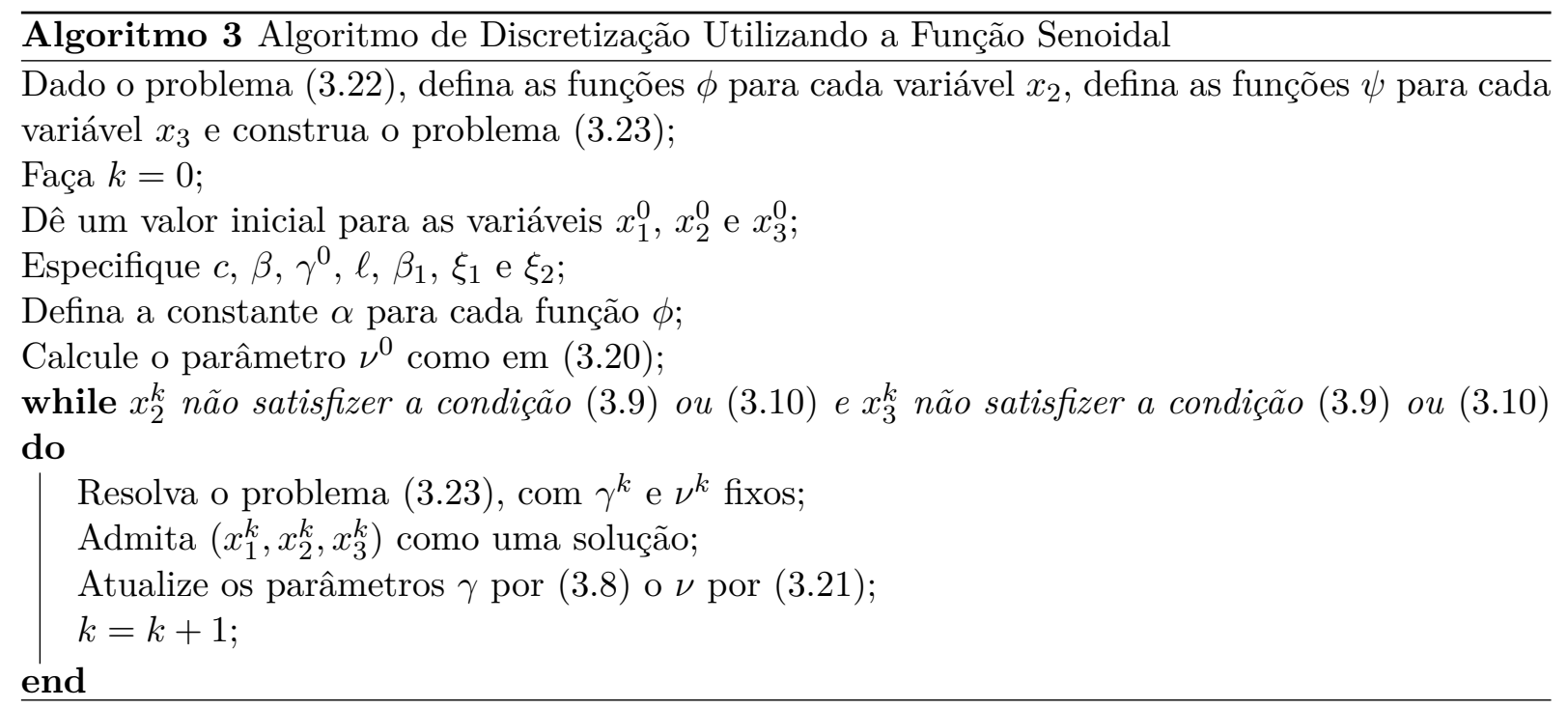

\subsection{Função Sigmoidal}

Problemas de otimização não linear com variáveis binárias existem em grande quantidade no mundo real, no entanto, existem poucos métodos de solução de sucesso. Isto acontece devido a complexidade de um problema deste tipo, por exemplo, até mesmo um dos casos mais simples com função objetivo quadrática e restrições lineares é NP-hard (MURRAY; NG, 2008). Esta seção descreve uma estratégia heurística, utilizando uma função sigmoidal, para o tratamento de variáveis binárias de um problema de otimização não linear.

Considere o seguinte problema PNL com variáveis binárias:

$$
\begin{array}{lll}
\min & f(x) & \\
\text { s.a: } & g_{i}(x)=0 & i=1, \cdots, p \\
& h_{i}(x) \leq 0 & i=1, \cdots, q \\
& x_{i} \in\{0,1\} & i=1, \cdots, n
\end{array}
$$

onde $x \in \mathbb{R}^{n}$ é um vetor de variáveis binárias; $f: \mathbb{R}^{n} \mapsto \mathbb{R} ; g: \mathbb{R}^{n} \mapsto \mathbb{R}^{p}$, com $p<n$; e $h: \mathbb{R}^{n} \mapsto \mathbb{R}^{q}$ O tratamento das variáveis binárias de um problema como (3.24) pode ser feito utilizando uma função sigmoidal definida como: 


$$
\chi(y)=\frac{e^{\tau y}-1}{e^{\tau y}+1},
$$

onde $\tau$ é a inclinação da função sigmoidal. Quanto maior o valor atribuído a $\tau$ maior a inclinação da sigmoidal tendendo a um ângulo reto. Este efeito é apresentado na Figura 3.14.

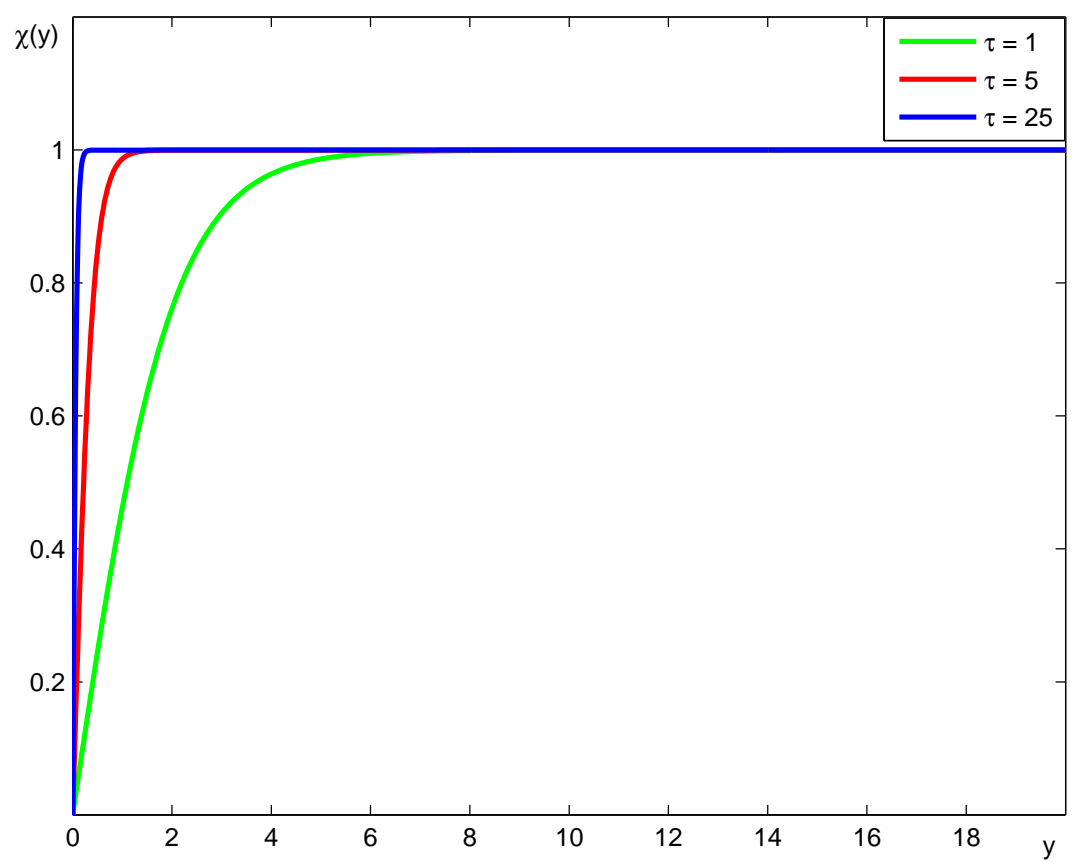

Figura 3.14 - Representação gráfica da função (3.25), para diferentes valores de $\tau$.

A função $\chi(y)$, dependo do valor de $\tau$, resulta em valores muito próximos de 0 ou 1 , representando assim as variáveis binárias. O argumento $y$ da função $\chi$ pertence ao intervalo $[0,20]$, este intervalo foi adotado heuristicamente. Maiores informações sobre esta função sigmoidal podem ser encontradas na referência (OLIVEIRA et al., 2005).

Incorporando a função $\chi$ mostrada em (3.25) na função objetivo do problema (3.24) temos o seguinte problema modificado:

$$
\begin{array}{lll}
\min & f(\chi(y)) & \\
\text { s.a: } & g_{i}(\chi(y))=0 & i=1, \cdots, p \\
& h_{i}(\chi(y)) \leq 0 & i=1, \cdots, q \\
& 0 \leq y_{i} \leq 20 & i=1, \cdots, n
\end{array}
$$

onde $\chi\left(y_{i}\right)$ é a função que representa a variável binária, tal que $\chi\left(y_{i}\right)=x_{i}$, e que nesta modelagem passa a ser contínua. 


\subsubsection{Algoritmo Proposto Utilizando a Função Sigmoidal}

Este algoritmo consiste na resolução de uma sequência de problemas modificados como (3.26) até que todas as variáveis associadas às variáveis binárias do problema original assumam valores binários.

A determinação de um valor inicial para o parâmetro $\tau$ é fundamental para que a solução do problema (3.26) seja encontrada. Para que o valor de $\tau$ seja determinado corretamente, propõe-se a resolução de uma série de problemas como (3.26) para diferentes valores de $\tau$, com $\tau$ crescendo gradualmente. O ajuste do crescimento de $\tau$ é dado por:

$$
\tau^{k+1}=\bar{c} \tau^{k}
$$

onde $\bar{c}$ é o fator de ajuste do crescimento de $\tau$, definido no intervalo $(1,10]$, e $k$ representa a $k$-ésima iteração do algoritmo. O processo de resoluções sucessivas do problema (3.26) e de ajuste do parâmetro $\tau$ continuam até que o critério de convergência do algoritmo seja satisfeito.

O critério de convergência do algoritmo consiste em avaliar se o valor da função $\chi\left(y_{i}\right)^{k}$ é binário na solução corrente. Se a maior diferença entre a função $\chi(y)^{k}$ e o binário mais próximo de $\chi(y)^{k}$ for menor que uma tolerância predeterminada, isto significa que o valor definido por $\chi\left(y_{i}\right)^{k}$ é suficientemente bom e o algoritmo é interrompido. Matematicamente, esse critério pode ser representado por:

$$
\left\|\chi(y)^{k}-\operatorname{Bin}^{k}\right\|_{\infty} \leq \xi_{3}
$$

onde $\operatorname{Bin}^{k}$ é o vetor de variáveis binárias (0 ou 1), na qual cada componente $i$ de $\operatorname{Bin}^{k}$ contém o binário mais próximo de cada componente $i$ de $\chi(y)^{k}$.

Outro critério aplicado consiste em avaliar os ajustes nas variáveis $\chi\left(y_{i}\right)$ entre duas iterações consecutivas. Se o maior ajuste em uma variável $\chi\left(y_{i}\right)$ for menor que uma tolerância predeterminada, o algoritmo é forçado a "arredondar" esta variável. Matematicamente, este critério pode ser representado por:

$$
\left\|\chi\left(y_{i}\right)^{k}-\chi\left(y_{i}\right)^{k-1}\right\|_{\infty}<\xi_{4}
$$

onde $\xi_{4}$ é a tolerância de ajuste das variáveis binárias entre duas iterações consecutivas. Caso o critério 3.29 seja verdadeiro, então é necessário verificar se a variável de controle associada a $\chi\left(y_{i}\right)$ mudou de valor com relação ao valor inicial atribuído a ela. Matematicamente, $\chi\left(y_{i}\right)$ assume valores da seguinte forma:

$$
\chi\left(y_{i}\right)^{k}= \begin{cases}0 & \text { se }\left\|x_{i}^{k}-x_{i}^{0}\right\|_{\infty} \leq \xi_{5} \\ 1 & \text { c.c. }\end{cases}
$$


O arredondamento (3.30) é necessário pois pode ocorrer de uma variável ser nula e a função $\chi(y)$ força-la a ser 1 , já que o problema modificado mascara esta restrição.

A resolução de um problema de PNLIM como (3.24) é realizada pelo Algoritmo 4.

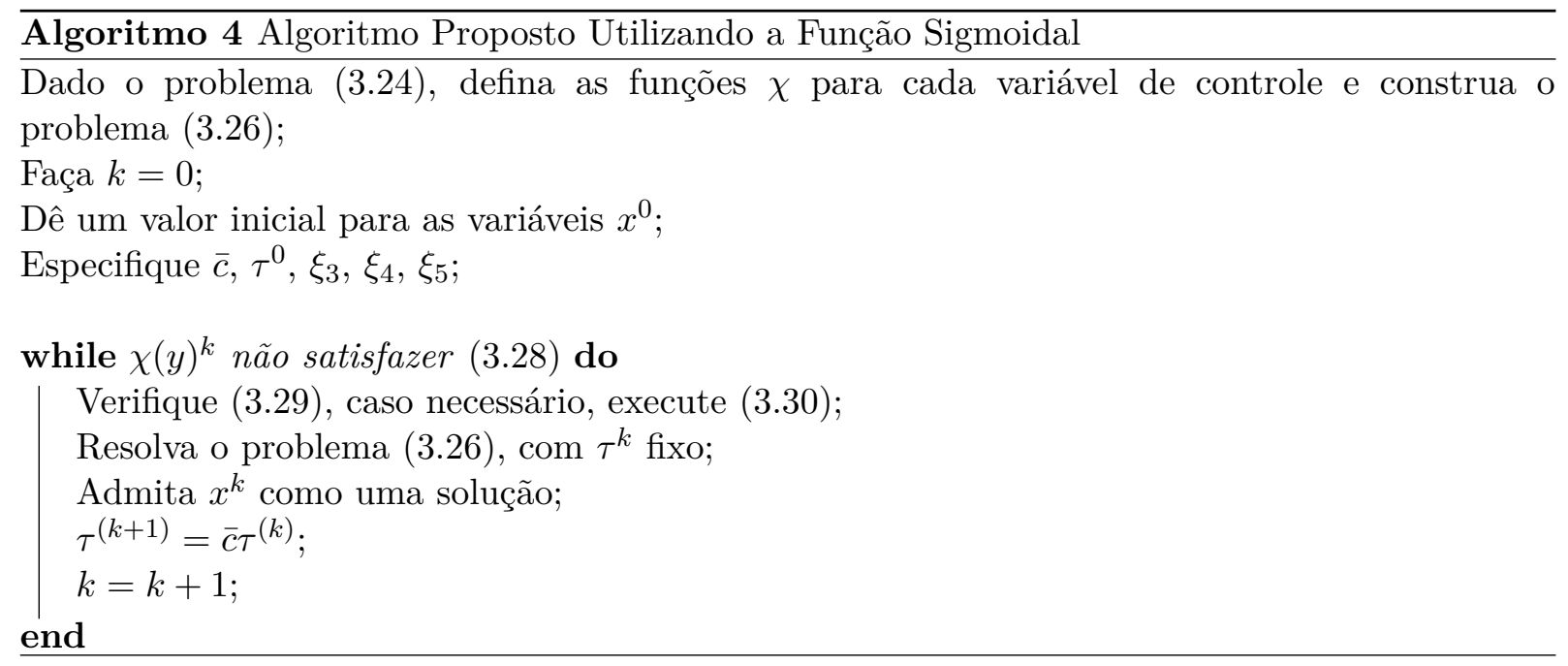

\subsection{Função Quadrática}

Para evitar a carga computacional imposta pelo problema PNL com muitas variáveis binárias, a estratégia, usando uma função quadrática, baseada em restrições de equilíbrio para lidar com as variáveis binárias é proposta. Está estratégia é considerada uma estratégia simples e trivial na modelagem das variáveis binárias e maiores informações podem ser encontradas em (MURRAY; NG, 2008).

Considere o problema PNL com variáveis binárias (3.24). Uma simples maneira de forçar $x_{i} \in\{0,1\}$ é transformando $x_{i}$ em uma variável contínua, adicionando ao problema (3.24) as restrições

$$
0 \leq x_{i} \leq 1 \quad i=1, \cdots, n
$$

e

$$
x_{i}\left(1-x_{i}\right)=0 \quad i=1, \cdots, n .
$$

É evidente que a restrição (3.32) é satisfeita apenas quando $x_{i}$ toma valores 0 ou 1 , sendo descrita pela Figura 3.15. 


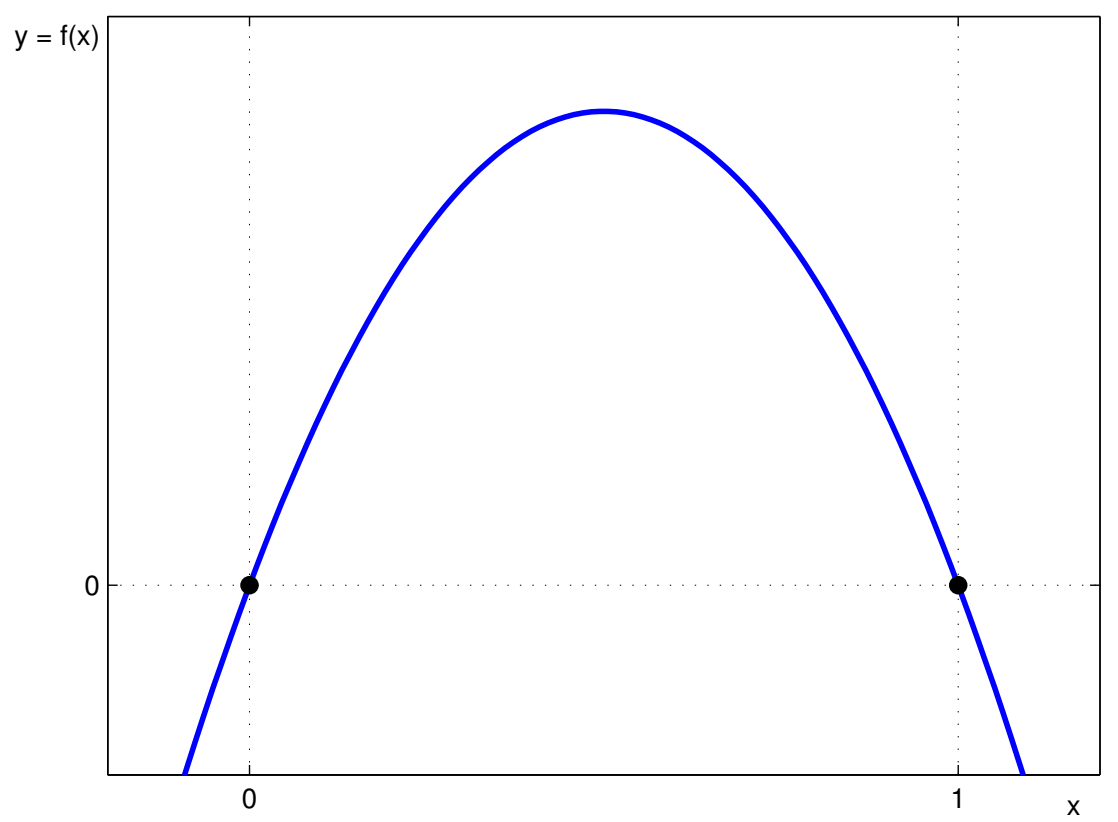

Figura 3.15 - Representação gráfica da restrição (3.32).

O problema (3.24) modificado pelas restrições (3.31) e (3.32) é dado por:

$$
\begin{array}{lll}
\min & f(x) & \\
\text { s.a: } & g_{i}(x)=0 & i=1, \cdots, p \\
& h_{i}(x) \leq 0 & i=1, \cdots, q \\
& x_{i}\left(1-x_{i}\right)=0 & i=1, \cdots, n \\
& 0 \leq x_{i} \leq 1 & i=1, \cdots, n
\end{array}
$$

Ao utilizar este tipo de abordagem deve-se considerar que encontrar o minimizador global neste caso é o oposto de encontrar um minimizador local, sendo muitas vezes impossível de se obter o ótimo global para um grande número de variáveis binárias.

No próximo capítulo, serão apresentados alguns conceitos básicos da Otimização Multiobjetivo Não Linear e os métodos da soma ponderada e $\epsilon$-restrito que serão utilizados na resolução do problema multiobjetivo. 


\section{Capítulo 4}

\section{Otimização Multiobjetivo Não Linear}

Neste Capítulo, apresentamos alguns conceitos básicos da Otimização Multiobjetivo Não Linear, como por exemplo o conceito de Otimalidade de Pareto. Apresentamos também dois métodos clássicos para a resolução do problema multiobjetivo, o método da soma ponderada e o método $\epsilon$-restrito. Os conceitos encontrados neste Capítulo foram baseados principalmente em (MIETTINEN, 1999).

\subsection{Conceitos}

O problema de otimização multiobjetivo pode ser escrito da seguinte forma:

$$
\begin{aligned}
\min & F(x)=\left[f_{1}(x), f_{2}(x), \cdots, f_{k}(x)\right]^{T} \\
\text { s.a: } & x \in S
\end{aligned}
$$

onde temos $k(\geq 2)$ funções objetivos $f_{i}: \mathbb{R}^{n} \rightarrow \mathbb{R}$. $\mathrm{O}$ vetor de variáveis de decisão $x=\left[x_{1}, x_{2}, \cdots, x_{n}\right]^{T}$ pertence ao conjunto da região factível (não vazio) $S$, que é um subconjunto do espaço de decisão $\mathbb{R}^{n}$. $S$ é composto pelas restrições impostas ao problema.

Se não houver um conflito entre as funções objetivo elas são separáveis, então a solução pode ser encontrada quando todas as funções objetivos alcançam seus ótimos. Aqui abordaremos o caso onde as funções objetivo são conflitantes. Na sequência, denotamos a imagem da região factível $S$ por $Z=(F(S))$ e vamos chamar de região objetivo viável, que nada mais é do que um subconjunto do espaço dos objetivos $\mathbb{R}^{k}$. Os elementos de $Z$ são chamados vetores objetivos ou vetores critérios e são denotados por $F(x)$ ou $z=\left[z_{1}, z_{2}, \cdots, z_{k}\right]^{T}$, onde $z_{i}=f_{i}(x)$ para todo $i=1,2, \cdots, k$ valores objetivos. Para maior clareza e simplicidade do tratamento assumimos que todas as funções objetivos devem ser minimizadas. Caso alguma função objetivo $f_{i}$ deva ser maximizada, podemos de forma equivalente minimizar a função $-f_{i}$. 


\subsubsection{Otimalidade de Pareto}

Nesta seção, nós lidamos com um conceito fundamental na otimização chamado otimalidade. Em problemas de otimização com um único objetivo, o foco principal está no espaço de variáveis de decisão. No contexto multiobjetivo estamos, muitas vezes, mais interessados no espaço de objetivos. Para começar, o espaço de objetivos é geralmente de uma dimensão menor do que o espaço de variáveis de decisão. Além disso, os valores objetivos são usados abaixo na definição de otimalidade.

Por causa da possível contradição e incomensurabilidade das funções objetivos, não é possível encontrar uma única solução que seria ideal para todos os objetivos simultaneamente. Problemas de otimização multiobjetivo estão de certo modo mal definidos. Não há qualquer ordenamento natural no espaço de objetivos, existe apenas um ordenamento parcial.

De qualquer forma, alguns dos vetores objetivos podem ser extraídos para serem examinados. Tais vetores são aqueles em que nenhum dos componentes podem ser melhorados sem que haja uma deterioração de pelo menos um dos outros componentes. Edgeworth apresentou esta definição em 1881, no entanto, esta definição é chamada Pareto-Ótima (Ótimo de Pareto) depois que o economista e sociólogo Franco-Italiano Vilfredo Pareto generalizou esta definição em 1896. Uma definição mais formal da Otimalidade de Pareto é apresentada a seguir.

Definição 4.1.1. Um vetor de decisão $x^{*} \in S$ é um Ótimo de Pareto se não existe nenhum outro vetor de decisão $x \in S$ tal que $f_{i}(x) \leq f_{i}\left(x^{*}\right)$ para todo $i=1, \cdots, k$ e $f_{j}(x)<f_{j}\left(x^{*}\right)$ para pelo menos um $\mathrm{j}$.

Um vetor objetivo (solução) $z^{*} \in Z$ é um Ótimo de Pareto se não existe nenhum outro vetor objetivo $z \in Z$ tal que $z_{i} \leq z_{i}^{*}$ para todo $i=1, \cdots, k$ e $z_{j}<z_{j}^{*}$ para pelo menos um j; ou equivalentemente, $z^{*}$ é um Ótimo de Pareto se o vetor de decisão correspondente é um Ótimo de Pareto.

Há geralmente uma infinidade de soluções ótimas de Pareto. Podemos mencionar tanto um conjunto de soluções ótimas de Pareto quanto um conjunto ótimo de Pareto. Este conjunto pode ser não convexo e não conectado. A Figura 4.1, mostra um exemplo de mapeamento entre os espaços S e Z, e as soluções ótimas de Pareto .

\subsubsection{Tomando Decisões}

Matematicamente, cada ótimo de Pareto é uma solução igualmente aceitável para o problema de otimização multiobjetivo. No entanto, é geralmente desejável obter um único ponto como solução. Ao selecionar uma solução do conjunto de soluções ótimas de Pareto necessita-se de informações que não estão contidas nas funções objetivo. Isto ocorre porque, em comparação com a otimização mono-objetivo, um novo elemento é adicionado em otimização multiobjetivo. Necessita-se que alguém tome a decisão sobre qual solução escolher. O decisor é uma pessoa (ou um grupo de pessoas), que é suposto ter uma melhor visão sobre o problema e que pode expressar relações de preferências entre as diferentes soluções. Normalmente, o decisor é responsável pela solução final. 


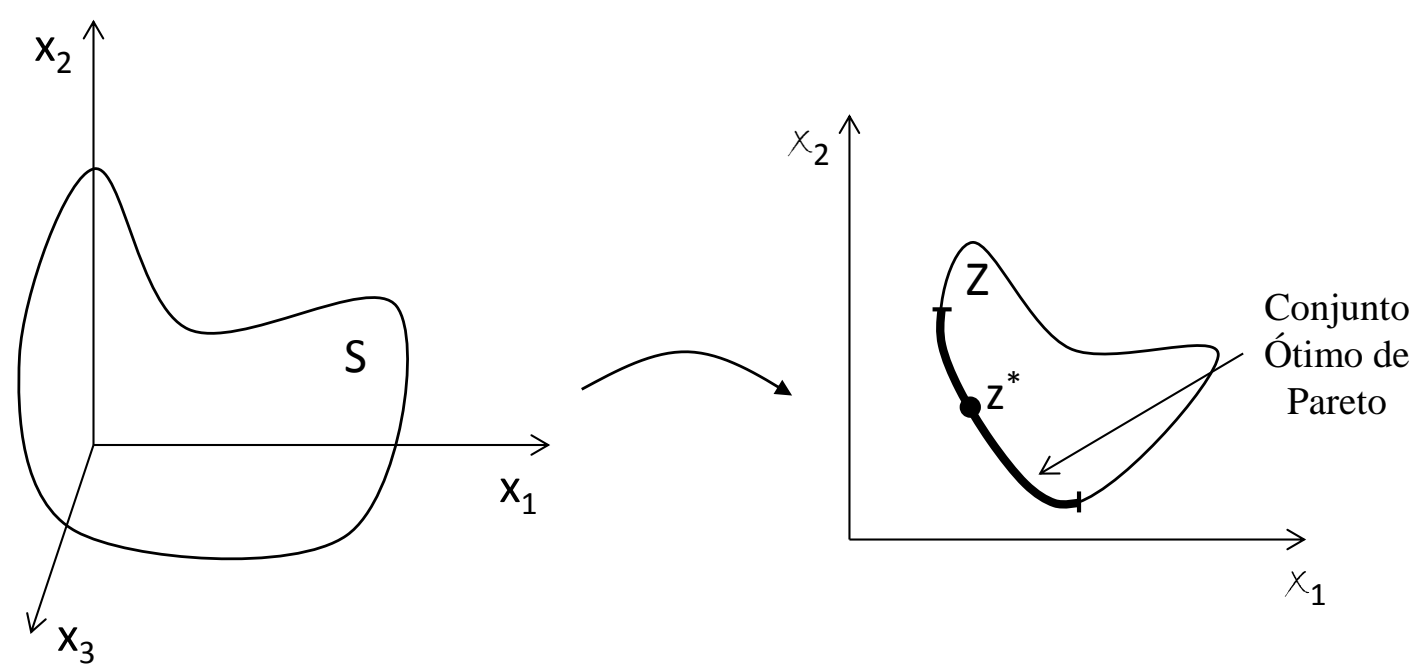

Figura 4.1 - Os conjuntos S e Z e as soluções ótimas de Pareto.

Para resolver um problema multiobjetivo de otimização é necessário a cooperação do decisor e de um analista. O analista seria uma pessoa ou um programa de computador responsável pelo lado matemático do processo de solução, gerando informações para o decisor levar em consideração e escolher a melhor solução de acordo com as suas preferências.

\subsection{Métodos de Otimização Multiobjetivo}

Gerar soluções ótimas de Pareto desempenha um papel importante na otimização multiobjetivo, e matematicamente, um problema é solucionado quando o conjunto ótimo de Pareto é encontrado. O termo vetor de otimização é por vezes utilizado para designar o problema de identificar o melhor conjunto de Pareto, entretanto, isto não é sempre suficiente pois deseja-se obter apenas uma solução. Isto significa que temos de encontrar uma maneira de colocar as soluções ótimas de Pareto em uma ordem completa. É por isto que precisamos de um decisor e de sua estrutura de preferências.

A existência de objetivos conflitantes torna o processo de encontrar soluções viáveis que otimizem simultaneamente todos os objetivos um grande desafio da Otimização Multiobjetivo. Com isto, os métodos de Otimização Multiobjetivo são compostos de duas etapas básicas: determinação de soluções e tomada de decisão (por parte do decisor).

Para muitos autores, os métodos de Otimização Multiobjetivo podem ser classificados em três categorias: 1) Método a-priori ou tomada de decisão antes da busca; 2) Métodos a-posteriori ou tomada de decisão depois da busca; e 3) Método iterativo ou tomada de decisão durante a busca. A seguir, apresenta-se uma breve explicação sobre cada uma das três categorias. 


\subsubsection{Métodos a-priori}

Os métodos considerados a-priori, são aqueles em que o decisor participa da busca de soluções antes da resolução do problema. São duas as maneiras de atribuir elementos de preferências para os objetivos: 1) Pode-se combinar os objetivos em um único objetivo, explicitando a preferência de cada objetivo através de pesos, podendo desta forma aplicar os métodos tradicionais de otimização mono-objetivo; e 2) Classificar os objetivos em ordem de preferências, assim o problema é resolvido considerando apenas o primeiro objetivo em ordem de preferência, depois o problema é resolvido para o segundo objetivo, e o processo se repete até que todos os objetivos sejam utilizados.

\subsubsection{Métodos a-posteriori}

Neste caso, realiza-se uma busca pelas soluções Pareto-ótimas e somente depois é realizada a tomada de decisão. A busca pelas soluções Pareto-ótimas é realizada considerando que todos os objetivos tem a mesma relevância para o problema. Após a realização da busca, obtém-se o conjunto de soluções Pareto-ótimas no qual o decisor deve selecionar a solução mais adequada. A desvantagem deste tipo de método é o alto custo computacional para encontrar o conjunto de soluções Pareto-ótimas.

\subsubsection{Métodos iterativos}

Os métodos iterativos sofrem uma interferência durante o processo de busca de soluções. O decisor intervém durante o processo de busca do conjunto Pareto-ótimo, atribuindo suas preferências e orientado a busca para regiões de maior interesse a partir das soluções parciais obtidas. Este tipo de método se torna inviável, a partir do momento que ocorrem muitas intervenções do decisor (fator humano).

\subsection{Métodos Clássicos de Otimização Multiobjetivo}

Apresenta-se nesta seção dois métodos clássicos para a resolução de problemas de Otimização Multiobjetivo que escalarizam os objetivos formando um único objetivo. Neste caso, o método transforma o problema multiobjetivo em um problema modificado mono-objetivo, o qual pode utilizar métodos da otimização mono-objetivo para encontrar uma solução adequada.

\subsubsection{Método da soma ponderada}

No método da soma ponderada, a ideia consiste em associar cada função objetivo com um coeficiente de ponderação (pesos) e minimizar a soma ponderada dos objetivos. Deste modo, as múltiplas funções objetivos são transformadas em uma única função objetivo, ou seja, o problema de otimização multiobjetivo é modificado pelo método da soma ponderada tornando-se um problema de otimização mono-objetivo. Supõe-se que os coeficientes de ponderação $\omega_{i}$ são 
números reais tais que $\omega_{i} \geq 0$ para todo $\forall i=1, \cdots, k$. Considera-se também, que os pesos são normalizados, isto é $\sum_{i=1}^{k} \omega_{i}=1$.

Considere o problema (4.1) de otimização multiobjetivo, a modificação deste problema pelo método da soma ponderada, é dada pelo problema ponderado (4.2):

$$
\begin{array}{ll}
\min & F(x)=\sum_{i=1}^{k} \omega_{i} f_{i}(x) \\
\text { s.a: } & x \in S
\end{array}
$$

onde $\omega_{i} \geq 0$ para todo $\forall i=1, \cdots, k$ e $\sum_{i=1}^{k} \omega_{i}=1$

\subsubsection{Normalização}

No caso da soma ponderada é necessário que as funções objetivos do problema sejam normalizadas para que não ocorra nenhuma preferência de uma sobre outra que tenha menor peso na função objetivo ponderada. Com isso a normalização das funções objetivos de um problema de Otimização Multiobjetivo é feita da seguinte maneira:

$$
f_{i}(x)^{n o r}=\frac{\left(f_{i}(x)-f_{i}(x)^{\min }\right)}{\left(f_{i}(x)^{\text {max }}-f_{i}(x)^{\min }\right)}
$$

onde $f_{i}(x)$ é uma função objetivo, $f_{i}(x)^{\text {min }}$ é o valor mínimo encontrado para esta função objetivo e $f_{i}(x)^{\text {max }}$ é o valor máximo encontrado para esta função objetivo. A normalização deve ser feita para todos os objetivos i.

\subsubsection{Método $\epsilon$-restrito}

No método $\epsilon$-restrito uma das funções objetivos é selecionada para ser otimizada e todas as outras funções objetivos são convertidas em restrições de desigualdade definindo um limite superior $\epsilon$ para cada uma delas.

O problema (4.1) é modificado pelo método $\epsilon$-restrito da seguinte forma:

$$
\begin{array}{lll}
\min & f_{l}(x) & l \in 1, \cdots, k \\
\text { s.a: } & f_{j}(x) \leq \epsilon_{j} & j=1, \cdots, k \text { e } j \neq l ; \\
& x \in S &
\end{array}
$$

onde cada $\epsilon_{j}$ representa um valor máximo para o valor de $f_{j}(x)$.

Essa abordagem pode ser usada em problemas com espaço de objetivos convexo, não convexo ou discreto. A construção do conjunto Pareto-ótimo por esta abordagem depende de variar o valor de $\epsilon_{j}$, porém não é garantido que o subconjunto encontrado tenha soluções, podendo este ser vazio, dependo dos valores escolhidos de $\epsilon_{j}$. 
No próximo capítulo apresentam-se a modelagem, algoritmos e estratégias para o tratamento os problemas de FPO, principalmente o multiobjetivo, com os quais serão realizados os testes. 


\section{Capítulo 5}

\section{Modelos e Algoritmos}

Neste capítulo apresentamos o modelo para o problema de Limitação do Número de Ações de Controles (2.8) modificado pela função quadrática para o tratamento das variáveis binárias e pela função de discretização polinomial. Apresentamos o modelo de FPO Multiobjetivo com tap de transformadores contínuos e elementos shunt fixos (2.14) modificado pelo método da soma ponderada, apresentado no Capítulo 4, e pela função sigmoidal, descrita no Capítulo 3, para o tratamento das variáveis binárias associadas as ações de controle, e o algoritmo para resolução desta estratégia. Apresentamos o modelo de FPO Multiobjetivo com tap de transformadores contínuos e elementos shunt fixos (2.14) modificado pelo método $\epsilon$-restrito, apresentado no Capítulo 4, e pela função quadrática, descrita no Capítulo 3, para o tratamento das variáveis discretas. Apresentamos o modelo de FPO Multiobjetivo com tap de transformadores discretos e elementos shunt fixos (5.7) modificado pelo método $\epsilon$-restrito, pela função quadrática para o tratamento das variáveis discretas, e pela função polinomial, descrita no Capítulo 3, para o tratamento das variáveis discretas. Por fim, o problema de FPO Multiobjetivo com tap de transformadores e elementos shunt discretos como em (2.15) é modificado pelo método da soma ponderada, pela função sigmoidal para o tratamento das variáveis binárias e pelas funções de discretização, descrita no Capítulo 3, para o tratamento das variáveis discretas.

\subsection{Limitação do Número de Ações de Controles em um problema de FPO}

O modelo (2.8) é modificado pela função quadrática para o tratamento das variáveis binárias e pela função de discretização polinomial gerando o modelo (5.1). 


$$
\begin{aligned}
& \min \quad P_{\text {perdas }}+\nu \sum_{k, m \in \mathcal{T}} \psi\left(t_{k m}\right) \\
& \text { s.a: } \quad P_{k}-\sum_{m \in \mathcal{V}_{k}} P_{k m}(V, \theta, t)=0 \quad \forall k \in \mathcal{G}^{\prime} \cup \mathcal{C} \\
& Q_{k}+Q_{k}^{s h}\left(V_{k}\right)-\sum_{m \in \mathcal{V}_{k}} Q_{k m}(V, \theta, t)=0 \quad \forall k \in \mathcal{C} \\
& Q_{G_{k}}^{\min } \leq Q_{G_{k}}(V, \theta, t) \leq Q_{G_{k}}^{\max } \quad \forall k \in \mathcal{G} \\
& V_{k}^{\text {min }} \leq V_{k} \leq V_{k}^{\text {max }} \quad \forall k \in \mathcal{B} \\
& t_{k m}^{\min } \leq t_{k m} \leq t_{k m}^{\max } \quad \forall k, m \in \mathcal{T} \\
& s_{1_{k}}\left(V_{k}^{\text {min }}-V_{k}^{0}\right) \leq\left(V_{k}-V_{k}^{0}\right) \leq s_{1_{k}}\left(V_{k}^{\max }-V_{k}^{0}\right) \quad \forall k \in \mathcal{G} \\
& s_{2_{k m}}\left(t_{k m}^{m i n}-t_{k m}^{0}\right) \leq\left(t_{k m}-t_{k m}^{0}\right) \leq s_{2_{k m}}\left(t_{k m}^{\max }-t_{k m}^{0}\right) \quad \forall k, m \in \mathcal{T} \\
& N^{\text {min }} \leq \sum_{k \in \mathcal{G}} s_{1_{k}}+\sum_{k, m \in \mathcal{T}} s_{2_{k m}} \leq N^{\max } \\
& s_{1_{k}}\left(1-s_{1_{k}}\right)=0 \quad k \in \mathcal{G} \\
& s_{2_{k m}}\left(1-s_{2_{k m}}\right)=0 \quad k, m \in \mathcal{T} \\
& 0 \leq s_{1_{k}} \leq 1 \quad k \in \mathcal{G} \\
& 0 \leq s_{2_{k m}} \leq 1 \quad k, m \in \mathcal{T}
\end{aligned}
$$

onde as especificações do problema(5.1) são iguais as do problema (2.8).

\subsection{FPO Multiobjetivo com tap de transformadores contínuos e elementos shunt fixos}

Considere o modelo de FPO Multiobjetivo com tap de transformadores contínuos e elementos shunt fixos (2.14) com objetivos de minimizar as perdas ativas na transmissão e minimizar o número de ações de controles. Este problema pode ser modificado em um problema mono-objetivo pelos métodos da soma ponderada (Seção 4.3.1) e do $\epsilon$-restrito (Seção 4.3.2). Desta forma, o problema multiobjetivo modificado pode ser resolvido por métodos de PNL. Na sequência apresentamos o FPO Multiobjetivo com tap de transformadores contínuos e elementos shunt fixos modificado pelo método da soma ponderada e posteriormente pelo método $\epsilon$-restrito.

\subsubsection{Soma Ponderada}

O problema de FPO Multiobjetivo com tap de transformadores contínuos e elementos shunt fixos (2.14) modificado pelo método da soma ponderada é representado pelo modelo (5.2). 


$$
\begin{aligned}
& \min \omega P_{\text {perdas }}+(1-\omega) N \\
& \text { s.a: } \quad P_{k}-\sum P_{k m}(V, \theta, t)=0 \quad \forall k \in \mathcal{G}^{\prime} \cup \mathcal{C} \\
& Q_{k}+Q_{k}^{s h}\left(V_{k}\right)-\sum_{m \in \mathcal{V}_{k}} Q_{k m}(V, \theta, t)=0 \quad \forall k \in \mathcal{C} \\
& Q_{G_{k}}^{\min } \leq Q_{G_{k}}(V, \theta, t) \leq Q_{G_{k}}^{\max } \quad \forall k \in \mathcal{G} \\
& V_{k}^{\text {min }} \leq V_{k} \leq V_{k}^{\max } \quad \forall k \in \mathcal{B} \\
& t_{k m}^{\min } \leq t_{k m} \leq t_{k m}^{\max } \quad \forall k, m \in \mathcal{T} \\
& s_{1_{k}}\left(V_{k}^{\text {min }}-V_{k}^{0}\right) \leq\left(V_{k}-V_{k}^{0}\right) \leq s_{1_{k}}\left(V_{k}^{\text {max }}-V_{k}^{0}\right) \quad \forall k \in \mathcal{G} \\
& s_{2_{k m}}\left(t_{k m}^{\min }-t_{k m}^{0}\right) \leq\left(t_{k m}-t_{k m}^{0}\right) \leq s_{2_{k m}}\left(t_{k m}^{\max }-t_{k m}^{0}\right) \quad \forall k, m \in \mathcal{T} \\
& s_{1_{k}} \in\{0,1\} \quad k \in \mathcal{G} \\
& s_{2_{k m}} \in\{0,1\} \quad k, m \in \mathcal{T}
\end{aligned}
$$

onde $\omega$ é o coeficiente de ponderação ou peso atribuído a cada uma das duas funções objetivos, $0<\omega<1$. As demais especificações do problema(5.2) são iguais as do problema (2.14).

O tratamento das variáveis binárias do problema (5.2) será feito pela função Sigmoidal descrita na Seção (3.4). Desta forma, o problema (5.2) é modificado de maneira a conter somente variáveis contínuas, da seguinte forma:

$$
\begin{aligned}
& \min \omega P_{\text {perdas }}+(1-\omega) \bar{N} \\
& \text { s.a: } \quad P_{k}-\sum_{m \in \mathcal{V}_{k}} P_{k m}(V, \theta, t)=0 \\
& Q_{k}+Q_{k}^{s h}\left(V_{k}\right)-\sum_{m \in \mathcal{V}_{k}} Q_{k m}(V, \theta, t)=0 \quad \forall k \in \mathcal{C} \\
& Q_{G_{k}}^{\min } \leq Q_{G_{k}}(V, \theta, t) \leq Q_{G_{k}}^{\max } \quad \forall k \in \mathcal{G} \\
& V_{k}^{\text {min }} \leq V_{k} \leq V_{k}^{\max } \quad \forall k \in \mathcal{B} \\
& t_{k m}^{\min } \leq t_{k m} \leq t_{k m}^{\max } \quad \forall k, m \in \mathcal{T} \\
& \chi\left(y_{1_{k}}\right)\left(V_{k}^{\min }-V_{k}^{0}\right) \leq\left(V_{k}-V_{k}^{0}\right) \leq \chi\left(y_{1_{k}}\right)\left(V_{k}^{\max }-V_{k}^{0}\right) \quad \forall k \in \mathcal{G} \\
& \chi\left(y_{2_{k m}}\right)\left(t_{k m}^{\min }-t_{k m}^{0}\right) \leq\left(t_{k m}-t_{k m}^{0}\right) \leq \chi\left(y_{2_{k m}}\right)\left(t_{k m}^{\max }-t_{k m}^{0}\right) \quad \forall k, m \in \mathcal{T} \\
& 0 \leq \chi\left(y_{1_{k}}\right) \leq 20 \quad k \in \mathcal{G} \\
& 0 \leq \chi\left(y_{2_{k m}}\right) \leq 20 \quad k, m \in \mathcal{T}
\end{aligned}
$$


onde

$$
\bar{N}=\left(\sum_{k \in \mathcal{G}} \chi\left(y_{1_{k}}\right)+\sum_{k, m \in \mathcal{T}} \chi\left(y_{2_{k m}}\right)\right)
$$

$\chi\left(y_{1_{k}}\right)$ é a função sigmoidal que representa a variável binária $s_{1_{k}}$, tal que $\chi\left(y_{1_{k}}\right)=s_{1_{k}} ; \chi\left(y_{2_{k m}}\right)$ é a função sigmoidal que representa a variável binária $s_{2_{k m}}$, tal que $\chi\left(y_{2_{k m}}\right)=s_{2_{k m}}$; nesta modelagem $s_{1_{k}}$ e $s_{2_{k m}}$ passam a ser contínuas através de $\chi\left(y_{1_{k}}\right)$ e $\chi\left(y_{2_{k m}}\right)$.

Apresentamos a seguir uma variação do Algoritmo 4, o qual consiste na resolução de uma sequência de problemas modificados como (5.3) até que todas as variáveis associadas às variáveis binárias do problema original assumam valores binários. As especificações do Algoritmo 5 são similares as do Algoritmo 4.

A resolução de um problema de PNL que determina o compromisso entre as perdas ativas na transmissão e o número mínimo de ações de controles como (5.3) é dada pelo Algoritmo 5.

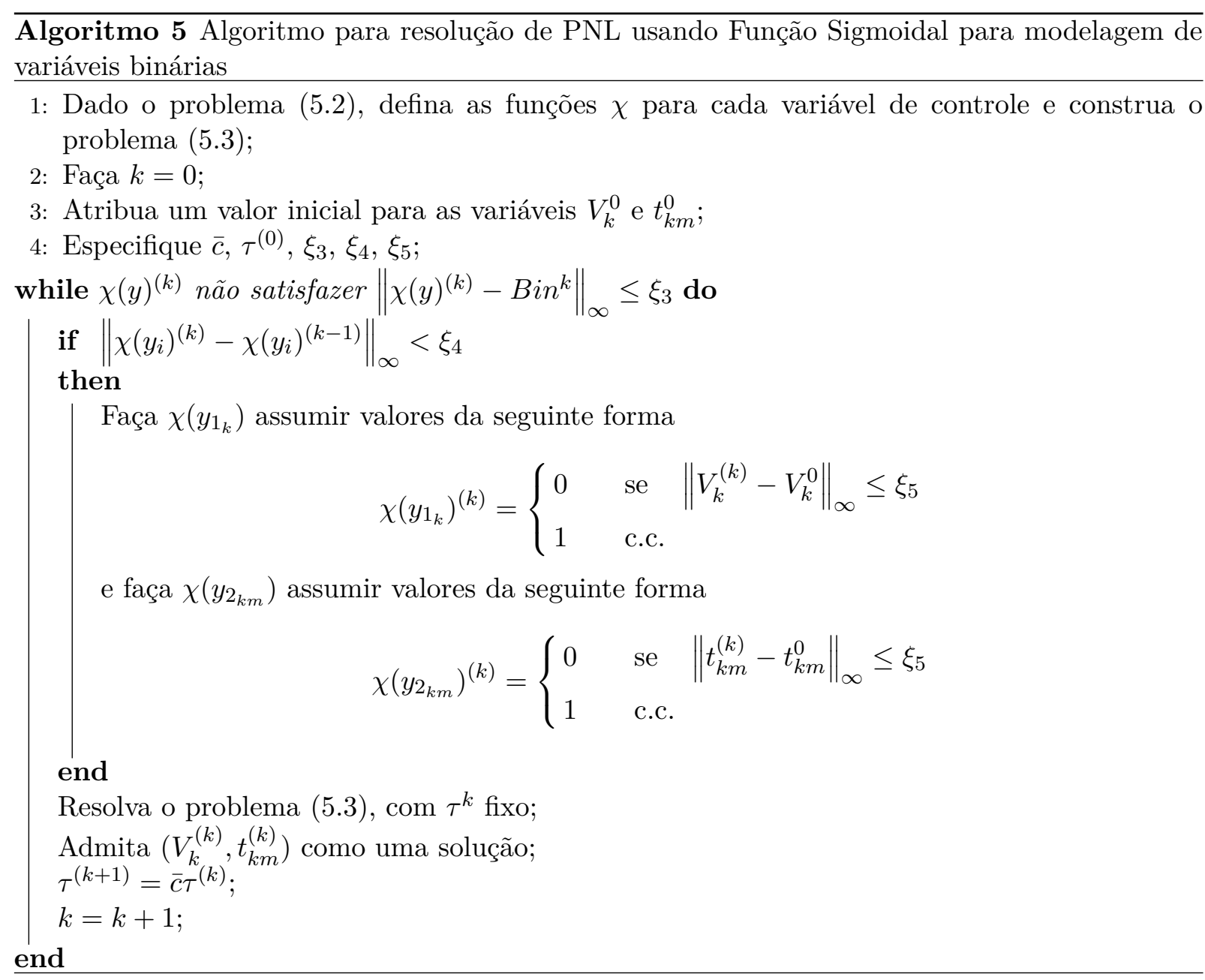




\subsubsection{Método $\epsilon$-restrito}

O modelo (5.5) apresenta modelo de FPO Multiobjetivo com tap de transformadores contínuos e elementos shunt fixos (2.14) modificado pelo método $\epsilon$-restrito.

$$
\begin{aligned}
& \text { min } \quad P_{\text {perdas }} \\
& \text { s.a: } \quad P_{k}-\sum_{m \in \mathcal{V}_{k}} P_{k m}(V, \theta, t)=0 \\
& Q_{k}+Q_{k}^{s h}\left(V_{k}\right)-\sum_{m \in \mathcal{V}_{k}} Q_{k m}(V, \theta, t)=0 \quad \forall k \in \mathcal{C} \\
& Q_{G_{k}}^{\min } \leq Q_{G_{k}}(V, \theta, t) \leq Q_{G_{k}}^{\max } \quad \forall k \in \mathcal{G} \\
& V_{k}^{\text {min }} \leq V_{k} \leq V_{k}^{\text {max }} \quad \forall k \in \mathcal{B} \\
& t_{k m}^{\min } \leq t_{k m} \leq t_{k m}^{\max } \quad \forall k, m \in \mathcal{T} \\
& s_{1_{k}}\left(V_{k}^{\text {min }}-V_{k}^{0}\right) \leq\left(V_{k}-V_{k}^{0}\right) \leq s_{1_{k}}\left(V_{k}^{\text {max }}-V_{k}^{0}\right) \quad \forall k \in \mathcal{G} \\
& s_{2_{k m}}\left(t_{k m}^{m i n}-t_{k m}^{0}\right) \leq\left(t_{k m}-t_{k m}^{0}\right) \leq s_{2_{k m}}\left(t_{k m}^{\max }-t_{k m}^{0}\right) \quad \forall k, m \in \mathcal{T} \\
& \sum_{k \in \mathcal{G}} s_{1_{k}}+\sum_{k, m \in \mathcal{T}} s_{2_{k m}}=\epsilon \\
& s_{1_{k}} \in\{0,1\} \\
& k \in \mathcal{G} \\
& s_{2_{k m}} \in\{0,1\}
\end{aligned}
$$

onde $\epsilon$ é valor que determina a quantidade de variáveis que podem atuar. Antes da resolução deste modelo determina-se o valor de $\epsilon$. Variando $\epsilon$ encontramos um trade-off entre as funções objetivos perdas ativas na transmissão e o número de ações de controle. As demais especificações deste sistema é igual as do problema (2.14).

As variáveis binárias do problema (5.5) podem ser tratadas pela função quadrática (Seção 3.5), e desta forma estas variáveis passam a ser consideradas contínuas como no problema modificado (5.6). 


$$
\begin{aligned}
& \min \quad P_{\text {perdas }} \\
& \text { s.a: } \quad P_{k}-\sum_{m \in \mathcal{V}_{k}} P_{k m}(V, \theta, t)=0 \\
& Q_{k}+Q_{k}^{s h}\left(V_{k}\right)-\sum_{m \in \mathcal{V}_{k}} Q_{k m}(V, \theta, t)=0 \\
& Q_{G_{k}}^{\min } \leq Q_{G_{k}}(V, \theta, t) \leq Q_{G_{k}}^{\max } \\
& V_{k}^{\text {min }} \leq V_{k} \leq V_{k}^{\text {max }} \\
& t_{k m}^{\min } \leq t_{k m} \leq t_{k m}^{\max } \\
& s_{1_{k}}\left(V_{k}^{\text {min }}-V_{k}^{0}\right) \leq\left(V_{k}-V_{k}^{0}\right) \leq s_{1_{k}}\left(V_{k}^{\max }-V_{k}^{0}\right) \\
& s_{2_{k m}}\left(t_{k m}^{m i n}-t_{k m}^{0}\right) \leq\left(t_{k m}-t_{k m}^{0}\right) \leq s_{2_{k m}}\left(t_{k m}^{\max }-t_{k m}^{0}\right) \quad \forall k, m \in \mathcal{T} \\
& \sum_{k \in \mathcal{G}} s_{1_{k}}+\sum_{k, m \in \mathcal{T}} s_{2_{k m}}=\epsilon \\
& s_{1_{k}}\left(1-s_{1_{k}}\right)=0 \\
& k \in \mathcal{G} \\
& s_{2_{k m}}\left(1-s_{2_{k m}}\right)=0 \\
& k, m \in \mathcal{T} \\
& 0 \leq s_{1_{k}} \leq 1 \\
& k \in \mathcal{G} \\
& 0 \leq s_{2_{k m}} \leq 1 \\
& \forall k \in \mathcal{G}^{\prime} \cup \mathcal{C} \\
& \forall k \in \mathcal{C} \\
& \forall k \in \mathcal{G} \\
& \forall k \in \mathcal{B} \\
& \forall k, m \in \mathcal{T} \\
& \forall k \in \mathcal{G} \\
& k, m \in \mathcal{T}
\end{aligned}
$$

\subsection{FPO Multiobjetivo com tap de transformadores discretos e elementos shunt fixos modelado pelo método $\epsilon$-restrito}

Considere o modelo de FPO multiobjetivo com tap de transformadores contínuos e as susceptâncias equivalentes aos bancos de capacitores e reatores shunts fixos (2.14), este modelo pode ser reescrito considerando os tap de transformadores discretos, da seguinte forma: 


$$
\begin{aligned}
& \min \quad\left[P_{\text {perdas }}, N\right]^{T} \\
& \text { s.a: } \quad P_{k}-\sum_{m \in \mathcal{V}_{k}} P_{k m}(V, \theta, t)=0 \\
& \forall k \in \mathcal{G}^{\prime} \cup \mathcal{C} \\
& Q_{k}+Q_{k}^{s h}\left(V_{k}\right)-\sum_{m \in \mathcal{V}_{k}} Q_{k m}(V, \theta, t)=0 \quad \forall k \in \mathcal{C} \\
& Q_{G_{k}}^{\min } \leq Q_{G_{k}}(V, \theta, t) \leq Q_{G_{k}}^{\max } \quad \forall k \in \mathcal{G} \\
& V_{k}^{\min } \leq V_{k} \leq V_{k}^{\max } \quad \forall k \in \mathcal{B} \\
& s_{1_{k}}\left(V_{k}^{\text {min }}-V_{k}^{0}\right) \leq\left(V_{k}-V_{k}^{0}\right) \leq s_{1_{k}}\left(V_{k}^{\max }-V_{k}^{0}\right) \quad \forall k \in \mathcal{G} \\
& s_{2_{k m}}\left(t_{k m}^{m i n}-t_{k m}^{0}\right) \leq\left(t_{k m}-t_{k m}^{0}\right) \leq s_{2_{k m}}\left(t_{k m}^{\max }-t_{k m}^{0}\right) \quad \forall k, m \in \mathcal{T} \\
& t_{k m} \in \mathcal{D}_{k m}^{t a p} \quad \forall k, m \in \mathcal{T} \\
& s_{1_{k}} \in\{0,1\} \\
& k \in \mathcal{G} \\
& s_{2_{k m}} \in\{0,1\}
\end{aligned}
$$

onde $\mathcal{D}_{k m}^{t a p}$ é o conjunto dos valores que a variável $t_{k m}$ pode assumir. As demais especificações são iguais as do problema (2.14).

O problema FPO Multiobjetivo, com tap de transformadores discretos e elementos shunt fixos (5.7), pode ser reescrito como um problema mono-objetivo pelo método $\epsilon$-restrito da seguinte forma.

$$
\begin{aligned}
& \min \quad P_{\text {perdas }} \\
& \text { s.a: } \quad P_{k}-\sum_{m \in \mathcal{V}_{k}} P_{k m}(V, \theta, t)=0 \quad \forall k \in \mathcal{G}^{\prime} \cup \mathcal{C} \\
& Q_{k}+Q_{k}^{s h}\left(V_{k}\right)-\sum_{m \in \mathcal{V}_{k}} Q_{k m}(V, \theta, t)=0 \quad \forall k \in \mathcal{C} \\
& Q_{G_{k}}^{\min } \leq Q_{G_{k}}(V, \theta, t) \leq Q_{G_{k}}^{\max } \quad \forall k \in \mathcal{G} \\
& V_{k}^{\text {min }} \leq V_{k} \leq V_{k}^{\max } \quad \forall k \in \mathcal{B} \\
& s_{1_{k}}\left(V_{k}^{\min }-V_{k}^{0}\right) \leq\left(V_{k}-V_{k}^{0}\right) \leq s_{1_{k}}\left(V_{k}^{\max }-V_{k}^{0}\right) \quad \forall k \in \mathcal{G} \\
& s_{2_{k m}}\left(t_{k m}^{\min }-t_{k m}^{0}\right) \leq\left(t_{k m}-t_{k m}^{0}\right) \leq s_{2_{k m}}\left(t_{k m}^{\max }-t_{k m}^{0}\right) \quad \forall k, m \in \mathcal{T} \\
& \sum_{k \in \mathcal{G}} s_{1_{k}}+\sum_{k, m \in \mathcal{T}} s_{2_{k m}}=\epsilon \\
& t_{k m} \in \mathcal{D}_{k m}^{t a p} \\
& \forall k, m \in \mathcal{T} \\
& s_{1_{k}} \in\{0,1\} \\
& k \in \mathcal{G} \\
& s_{2_{k m}} \in\{0,1\} \\
& k, m \in \mathcal{T}
\end{aligned}
$$


As variáveis binárias do problema (5.7) modificado pelo método $\epsilon$-restrito (5.8) podem ser modeladas pela função quadrática (Seção 3.5), como segue.

$$
\begin{aligned}
& \min \quad P_{\text {perdas }} \\
& \text { s.a: } \quad P_{k}-\sum_{m \in \mathcal{V}_{k}} P_{k m}(V, \theta, t)=0 \quad \forall k \in \mathcal{G}^{\prime} \cup \mathcal{C} \\
& Q_{k}+Q_{k}^{s h}\left(V_{k}\right)-\sum_{m \in \mathcal{V}_{k}} Q_{k m}(V, \theta, t)=0 \quad \forall k \in \mathcal{C} \\
& Q_{G_{k}}^{\min } \leq Q_{G_{k}}(V, \theta, t) \leq Q_{G_{k}}^{\max } \quad \forall k \in \mathcal{G} \\
& V_{k}^{\text {min }} \leq V_{k} \leq V_{k}^{\max } \quad \forall k \in \mathcal{B} \\
& s_{1_{k}}\left(V_{k}^{\min }-V_{k}^{0}\right) \leq\left(V_{k}-V_{k}^{0}\right) \leq s_{1_{k}}\left(V_{k}^{\max }-V_{k}^{0}\right) \quad \forall k \in \mathcal{G} \\
& s_{2_{k m}}\left(t_{k m}^{\min }-t_{k m}^{0}\right) \leq\left(t_{k m}-t_{k m}^{0}\right) \leq s_{2_{k m}}\left(t_{k m}^{\max }-t_{k m}^{0}\right) \quad \forall k, m \in \mathcal{T} \\
& \sum_{k \in \mathcal{G}} s_{1_{k}}+\sum_{k, m \in \mathcal{T}} s_{2_{k m}}=\epsilon \\
& s_{1_{k}}\left(1-s_{1_{k}}\right)=0 \quad k \in \mathcal{G} \\
& s_{2_{k m}}\left(1-s_{2_{k m}}\right)=0 \quad k, m \in \mathcal{T} \\
& t_{k m} \in \mathcal{D}_{k m}^{t a p} \quad \forall k, m \in \mathcal{T} \\
& 0 \leq s_{1_{k}} \leq 1 \quad k \in \mathcal{G} \\
& 0 \leq s_{2_{k m}} \leq 1 \quad k, m \in \mathcal{T}
\end{aligned}
$$

Já as variáveis discretas do problema de FPO (5.9) podem ser modeladas pelas funções de discretização Senoidal, Polinomial e Mista apresentadas no Capítulo 3.

\subsection{FPO Multiobjetivo com tap de transformadores e elementos shunt discretos}

Considere o problema de FPO Multiobjetivo com tap de transformadores e elementos shunt discretos como em (2.15). Podemos tratar o modelo (2.15) pelo método da soma ponderada, desta maneira o problema FPO Multiobjetivo com tap de transformadores e elementos shunt discretos é modificado em um problema mono-objetivo como em (5.10). 


$$
\begin{aligned}
& \min \omega P_{\text {perdas }}+(1-\omega) N \\
& \text { s.a: } \quad P_{k}-\sum_{m \in \mathcal{V}_{k}} P_{k m}(V, \theta, t)=0 \\
& Q_{k}+Q_{k}^{s h}\left(V_{k}, b_{k}^{s h}\right)-\sum_{m \in \mathcal{V}_{k}} Q_{k m}(V, \theta, t)=0 \quad \forall k \in \mathcal{C} \\
& Q_{G_{k}}^{\min } \leq Q_{G_{k}}\left(V, \theta, t, b_{k}^{s h}\right) \leq Q_{G_{k}}^{\max } \quad \forall k \in \mathcal{G} \\
& V_{k}^{\min } \leq V_{k} \leq V_{k}^{\max } \quad \forall k \in \mathcal{B} \\
& s_{1_{k}}\left(V_{k}^{\text {min }}-V_{k}^{0}\right) \leq\left(V_{k}-V_{k}^{0}\right) \leq s_{1_{k}}\left(V_{k}^{\max }-V_{k}^{0}\right) \quad \forall k \in \mathcal{G} \\
& s_{2_{k m}}\left(t_{k m}^{\min }-t_{k m}^{0}\right) \leq\left(t_{k m}-t_{k m}^{0}\right) \leq s_{2_{k m}}\left(t_{k m}^{\max }-t_{k m}^{0}\right) \quad \forall k, m \in \mathcal{T} \\
& s_{3_{k}}\left(b_{k}^{s h^{m i n}}-b_{k}^{s h^{0}}\right) \leq\left(b_{k}^{s h}-b_{k}^{s h^{0}}\right) \leq s_{3_{k}}\left(b_{k}^{s h^{\max }}-b_{k}^{s h^{0}}\right) \quad \forall k \in \mathcal{B}^{s h} \\
& t_{k m} \in \mathcal{D}_{k m}^{t a p} \quad \forall k, m \in \mathcal{T} \\
& b_{k}^{s h} \in \mathcal{D}_{k}^{s h} \\
& \forall k \in \mathcal{B}^{s h} \\
& s_{1_{k}} \in\{0,1\} \\
& k \in \mathcal{G} \\
& s_{2_{k m}} \in\{0,1\} \\
& k, m \in \mathcal{T} \\
& s_{3_{k}} \in\{0,1\}
\end{aligned}
$$

onde $\omega$ é o coeficiente de ponderação ou peso atribuído a cada uma das duas funções objetivos, $0<\omega<1$. As demais especificações do problema(5.10) são iguais as do problema (2.15).

Observe que o modelo (5.10) possui variáveis contínuas, discretas e binárias. As discretas são equivalentes aos taps de transformadores e aos bancos de capacitores e reatores shunts, enquanto as binárias estão associadas ao número de ações de controles. Deve ficar claro ainda que as funções objetivos do problema (2.15) são normalizadas no problema (5.10) como na Seção (4.3.1.1). Vamos utilizar a função Sigmoidal (3.4) para o tratamento das variáveis binárias do problema (5.10) como no modelo (5.11). 


$$
\begin{aligned}
& \min \omega P_{\text {perdas }}+(1-\omega) \bar{N} \\
& \text { s.a: } \quad P_{k}-\sum_{m \in \mathcal{V}_{k}} P_{k m}(V, \theta, t)=0 \\
& Q_{k}+Q_{k}^{s h}\left(V_{k}, b_{k}^{s h}\right)-\sum_{m \in \mathcal{V}_{k}} Q_{k m}(V, \theta, t)=0 \\
& Q_{G_{k}}^{\min } \leq Q_{G_{k}}\left(V, \theta, t, b_{k}^{s h}\right) \leq Q_{G_{k}}^{\max } \\
& V_{k}^{\min } \leq V_{k} \leq V_{k}^{\max } \\
& \chi\left(y_{1_{k}}\right)\left(V_{k}^{\min }-V_{k}^{0}\right) \leq\left(V_{k}-V_{k}^{0}\right) \leq \chi\left(y_{1_{k}}\right)\left(V_{k}^{\max }-V_{k}^{0}\right) \\
& \chi\left(y_{2_{k m}}\right)\left(t_{k m}^{\min }-t_{k m}^{0}\right) \leq\left(t_{k m}-t_{k m}^{0}\right) \leq \chi\left(y_{2_{k m}}\right)\left(t_{k m}^{\max }-t_{k m}^{0}\right) \\
& \chi\left(y_{3_{k}}\right)\left(b_{k}^{s h^{\min }}-b_{k}^{s h^{0}}\right) \leq\left(b_{k}^{s h}-b_{k}^{s h^{0}}\right) \leq \chi\left(y_{3_{k}}\right)\left(b_{k}^{s h^{\max }}-b_{k}^{s h^{0}}\right) \\
& 0 \leq \chi\left(y_{1_{k}}\right) \leq 20 \\
& 0 \leq \chi\left(y_{2_{k m}}\right) \leq 20 \\
& 0 \leq \chi\left(y_{3_{k}}\right) \leq 20 \\
& t_{k m} \in \mathcal{D}_{k m}^{t a p} \\
& b_{k}^{s h} \in \mathcal{D}_{k}^{s h} \\
& \forall k \in \mathcal{G}^{\prime} \cup \mathcal{C} \\
& \forall k \in \mathcal{C} \\
& \forall k \in \mathcal{G} \\
& \forall k \in \mathcal{B} \\
& \forall k \in \mathcal{G} \\
& \forall k, m \in \mathcal{T} \\
& \forall k \in \mathcal{B}^{\text {sh }} \\
& k \in \mathcal{G} \\
& k, m \in \mathcal{T} \\
& k \in \mathcal{B}^{s h} \\
& \forall k, m \in \mathcal{T} \\
& \forall k \in \mathcal{B}^{s h}
\end{aligned}
$$

sendo

$$
\bar{N}=\left(\sum_{k \in \mathcal{G}} \chi\left(y_{1_{k}}\right)+\sum_{k, m \in \mathcal{T}} \chi\left(y_{2_{k m}}\right)+\sum_{k \in \mathcal{B}^{s h}} \chi\left(y_{3_{k}}\right)\right)
$$

onde $\chi\left(y_{1_{k}}\right)$ é a função que representa a variável binária $s_{1_{k}}$, tal que $\chi\left(y_{1_{k}}\right)=s_{1_{k}} ; \chi\left(y_{2_{k m}}\right)$ é a função que representa a variável binária $s_{2_{k m}}$, tal que $\chi\left(y_{2_{k m}}\right)=s_{2_{k m}} ; \chi\left(y_{3_{k}}\right)$ é a função que representa a variável binária $s_{3_{k}}$, tal que $\chi\left(y_{3_{k}}\right)=s_{3_{k}}$; nesta modelagem $s_{1_{k}}, s_{2_{k m}}$ e $s_{3_{k}}$ passam a ser contínuas através de $\chi\left(y_{1_{k}}\right), \chi\left(y_{2_{k m}}\right)$ e $\chi\left(y_{3_{k}}\right)$.

Inicialmente podemos considerar as variáveis discretas do problema (5.11) como contínuas como em (5.13). 


$$
\begin{aligned}
& \min \omega P_{\text {perdas }}+(1-\omega) \bar{N} \\
& \text { s.a: } \quad P_{k}-\sum_{m \in \mathcal{V}_{k}} P_{k m}(V, \theta, t)=0 \\
& Q_{k}+Q_{k}^{s h}\left(V_{k}, b_{k}^{s h}\right)-\sum_{m \in \mathcal{V}_{k}} Q_{k m}(V, \theta, t)=0 \quad \forall k \in \mathcal{C} \\
& Q_{G_{k}}^{\min } \leq Q_{G_{k}}\left(V, \theta, t, b_{k}^{s h}\right) \leq Q_{G_{k}}^{\max } \quad \forall k \in \mathcal{G} \\
& V_{k}^{\text {min }} \leq V_{k} \leq V_{k}^{\max } \quad \forall k \in \mathcal{B} \\
& t_{k m}^{\min } \leq t_{k m} \leq t_{k m}^{\max } \quad \forall k, m \in \mathcal{T} \\
& b_{k}^{s h^{\min }} \leq b_{k}^{s h} \leq b_{k}^{s h^{\max }} \\
& \chi\left(y_{1_{k}}\right)\left(V_{k}^{\text {min }}-V_{k}^{0}\right) \leq\left(V_{k}-V_{k}^{0}\right) \leq \chi\left(y_{1_{k}}\right)\left(V_{k}^{\max }-V_{k}^{0}\right) \quad \forall k \in \mathcal{G} \\
& \chi\left(y_{2_{k m}}\right)\left(t_{k m}^{m i n}-t_{k m}^{0}\right) \leq\left(t_{k m}-t_{k m}^{0}\right) \leq \chi\left(y_{2_{k m}}\right)\left(t_{k m}^{\max }-t_{k m}^{0}\right) \quad \forall k, m \in \mathcal{T} \\
& \chi\left(y_{3_{k}}\right)\left(b_{k}^{s h^{m i n}}-b_{k}^{s h^{0}}\right) \leq\left(b_{k}^{s h}-b_{k}^{s h^{0}}\right) \leq \chi\left(y_{3_{k}}\right)\left(b_{k}^{s h^{\max }}-b_{k}^{s h^{0}}\right) \quad \forall k \in \mathcal{B}^{s h} \\
& 0 \leq \chi\left(y_{1_{k}}\right) \leq 20 \\
& k \in \mathcal{G} \\
& 0 \leq \chi\left(y_{2_{k m}}\right) \leq 20 \\
& k, m \in \mathcal{T} \\
& 0 \leq \chi\left(y_{3_{k}}\right) \leq 20 \\
& k \in \mathcal{B}^{\text {sh }}
\end{aligned}
$$

Vamos utilizar o Algoritmo 5 para resolver o problema (5.13). A única diferença do problema (5.3) para o problema (5.13) são as variáveis equivalentes aos elementos shunts, antes considerados fixos e agora considerados contínuos.

As variáveis discretas do problema (5.11) serão tratadas pelas funções de discretização Senoidal, Polinomial e a estratégia mista apresentadas no Capítulo 3. A seguir vamos descrever o modelo (5.11) modificado pelas funções de discretização de forma mais concisa: considere $\varphi$ podendo assumir tanto a função Senoidal quanto a Polinomial, então $\varphi\left(y_{i}\right)=\phi\left(y_{i}\right)$ como em (3.2) ou $\varphi\left(y_{i}\right)=\psi\left(y_{i}\right)$ como em (3.12). Assim, em função de $\varphi\left(y_{i}\right)$, podemos escrever o modelo (5.11) como um problema contínuo em (5.14). 


$$
\begin{aligned}
& \min \omega P_{\text {perdas }}+(1-\omega) \bar{N}+\varpi \bar{\varphi} \\
& \text { s.a: } \quad P_{k}-\sum_{m \in \mathcal{V}_{k}} P_{k m}(V, \theta, t)=0 \\
& Q_{k}+Q_{k}^{s h}\left(V_{k}, b_{k}^{s h}\right)-\sum_{m \in \mathcal{V}_{k}} Q_{k m}(V, \theta, t)=0 \quad \forall k \in \mathcal{C} \\
& Q_{G_{k}}^{\min } \leq Q_{G_{k}}\left(V, \theta, t, b_{k}^{s h}\right) \leq Q_{G_{k}}^{\max } \quad \forall k \in \mathcal{G} \\
& V_{k}^{\min } \leq V_{k} \leq V_{k}^{\max } \quad \forall k \in \mathcal{B} \\
& t_{k m}^{\min } \leq t_{k m} \leq t_{k m}^{\max } \quad \forall k, m \in \mathcal{T} \\
& b_{k}^{s h^{\text {min }}} \leq b_{k}^{s h} \leq b_{k}^{s h^{\max }} \quad \forall k \in \mathcal{B}^{s h} \\
& \chi\left(y_{1_{k}}\right)\left(V_{k}^{\text {min }}-V_{k}^{0}\right) \leq\left(V_{k}-V_{k}^{0}\right) \leq \chi\left(y_{1_{k}}\right)\left(V_{k}^{\max }-V_{k}^{0}\right) \quad \forall k \in \mathcal{G} \\
& \chi\left(y_{2_{k m}}\right)\left(t_{k m}^{\min }-t_{k m}^{0}\right) \leq\left(t_{k m}-t_{k m}^{0}\right) \leq \chi\left(y_{2_{k m}}\right)\left(t_{k m}^{\max }-t_{k m}^{0}\right) \quad \forall k, m \in \mathcal{T} \\
& \chi\left(y_{3_{k}}\right)\left(b_{k}^{s h^{\text {min }}}-b_{k}^{s h^{0}}\right) \leq\left(b_{k}^{s h}-b_{k}^{s h^{0}}\right) \leq \chi\left(y_{3_{k}}\right)\left(b_{k}^{s h^{\max }}-b_{k}^{s h^{0}}\right) \quad \forall k \in \mathcal{B}^{s h} \\
& 0 \leq \chi\left(y_{1_{k}}\right) \leq 20 \quad k \in \mathcal{G} \\
& 0 \leq \chi\left(y_{2_{k m}}\right) \leq 20 \quad k, m \in \mathcal{T} \\
& 0 \leq \chi\left(y_{3_{k}}\right) \leq 20 \quad k \in \mathcal{B}^{\text {sh }}
\end{aligned}
$$

onde $\varpi$ é o parâmetro de penalidade que controla a amplitude da função $\varphi$ e

$$
\bar{\varphi}=\left(\sum_{k, m \in \mathcal{T}} \varphi\left(t_{k m}\right)+\sum_{k \in \mathcal{B}^{s h}} \varphi\left(b_{k}^{s h}\right)\right) .
$$

\subsubsection{Algoritmos Propostos Utilizando a Função Sigmoidal e as Funções de Discretização}

O algoritmo proposto consiste em dois estágios: 1) decisão do controle e 2) discretização. Desta forma, no primeiro estágio ocorre a resolução de uma sequência de problemas modificados (5.13) até que todas as variáveis associadas às variáveis binárias do problema original assumam valores binários. No segundo estágio, uma sequência de problemas modificados (5.14) são resolvidos até discretizar todas as variáveis associadas às variáveis discretas do problema original e posteriormente redefinem-se as ações de controles resultantes.

Inicialmente, descreve-se o algoritmo correspondente ao primeiro estágio, ou seja, o algoritmo correspondente a decisão do controle. E em seguida apresenta-se o algoritmo que engloba a decisão do controle e a discretização.

O Algoritmo 6 apresentado a seguir é uma leve modificação do Algoritmo 5. A diferença entre os Algoritmos 5 e 6 consiste em que o primeiro a ser definido para um problema de FPO onde os elementos shunts de barra são considerados fixos e o segundo a ser definido para um problema de FPO onde os elementos shunts de barra são considerados contínuos. 


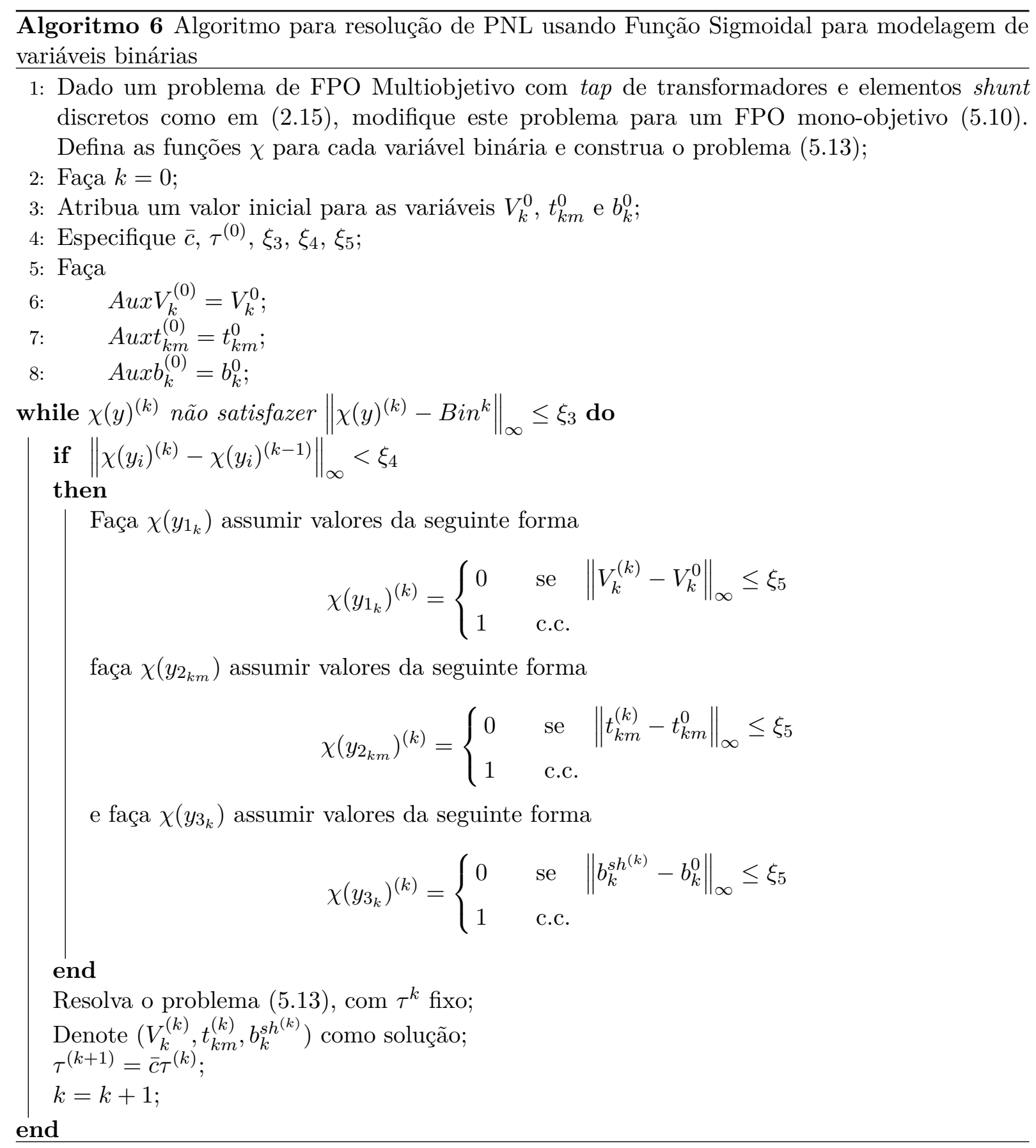

O ajuste do crescimento de $\varpi$, da função de discretização, adotada no Algoritmo 7, é dado por:

$$
\varpi^{k+1}=\hbar \varpi^{k}
$$

onde $\hbar$ é o fator de ajuste do crescimento de $\varpi$. Quando o modelo (5.14) é modificado pela função senoidal então $\varphi=\phi, \hbar=c$ e $\varpi=\gamma$. Quando o modelo (5.14) é modificado pela função polinomial então $\varphi=\psi, \hbar=\ell$ e $\varpi=\nu$. Quando o modelo (5.14) é modificado pela estratégia 
mista então os taps de transformadores são modelados pela função senoidal e os elementos shunts de barra são modelados pela função polinomial.

A resolução de um problema de PNL que determina o trade-off entre as perdas ativas na transmissão e o número mínimo de ações de controles como (5.13) é dada pelo Algoritmo 6 . A discretização é englobada como uma segunda tarefa, após a execução do Algoritmo 6. Esse processo é descrito como no diagrama da Figura 5.1. Na sequência definimos o Algoritmo 7 para a resolução de um problema PNL que determina o trade-off entre as perdas ativas na transmissão e o número mínimo de ações de controles.

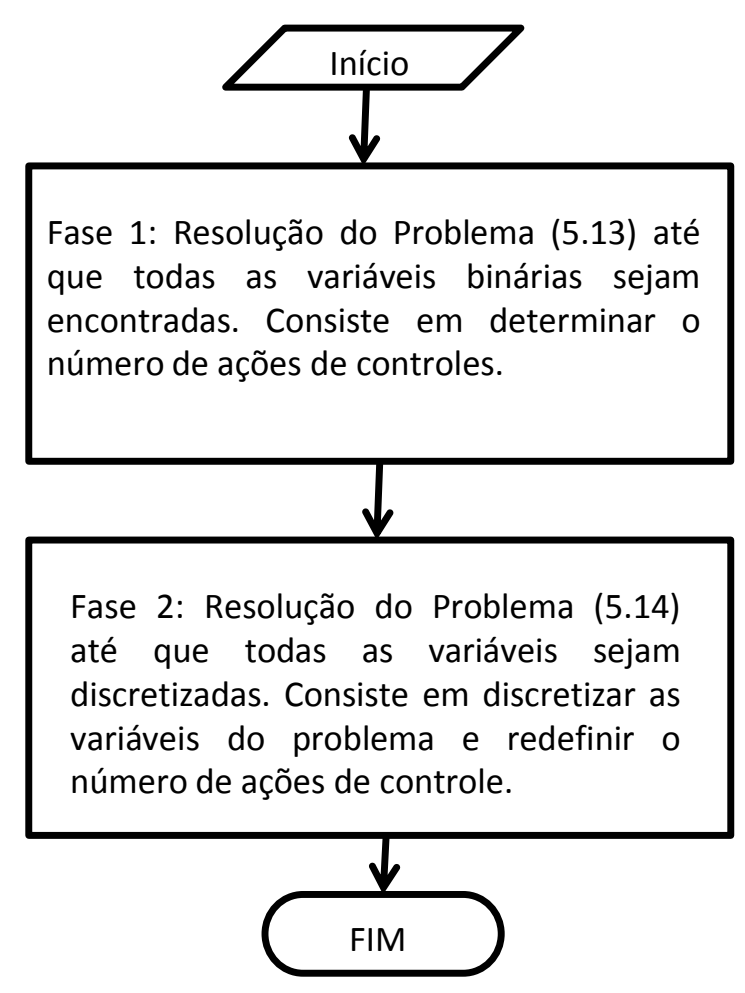

Figura 5.1 - Representação do Algoritmo 7.

O Algoritmo 7 é um algoritmo multiestágio proposto para a resolução do problema de FPO multiobjetivo com taps de transformadores e bancos de capacitores e reatores shunts discretos. Inicialmente o problema multiobjetivo é modificado pelo método da soma ponderada se tornando um problema mono-objetivo. Na primeira fase do algoritmo, as variáveis discretas são consideradas contínuas e as variáveis binárias são tratadas por uma função sigmoidal, determinando-se o número de ações de controles. Na segunda fase, as variáveis discretas são discretizadas pelas funções Senoidal, Polinomial ou Mista. A segunda fase, inicia-se com os valores encontrados pela primeira fase, e ao final de cada iteração da discretização verificam-se de fato quais controles atuaram. Desta forma, os controles encontrados na primeira fase podem não corresponder com os da segunda fase. Na prática, poucos controles mudam da primeira para a segunda fase, e geralmente está relacionado com as mudanças impostas pela discretização dos elementos shunts. Deve-se atentar ainda que o Algoritmo 7 encontra uma solução factível para o 
Algoritmo 7 Algoritmo Proposto para resolução de PNL usando Função Sigmoidal para modelagem de variáveis binárias e as funções de discretização

1: Dado um problema de FPO Multiobjetivo com tap de transformadores e elementos shunt discretos como em (2.15), modifique este problema para um FPO mono-objetivo (5.10). Defina as funções $\chi$ para cada variável binária e as funções $\varphi$ para cada variável discreta do problema (5.11). Construa os problemas (5.13) e (5.14).

Fase 1: Resolução do problema (5.13) para determinar o número de ações de controle do problema.

2: Resolva o problema (5.13) pelo Algoritmo 6;

3: Faça $\bar{k}=k$.

Fase 2: Resolução do problema (5.14) para discretizar as variáveis e redefinir o número de ações de controle do problema.

4: Faça $k=0$;

5: Assuma as variáveis calculadas pelo Algoritmo 5 como as variáveis iniciais $V_{k}^{0}, t_{k m}^{0}$ e $b_{k}^{0}$ do problema (5.14);

6: Dê um valor inicial para o parâmetro $\varpi^{0}$;

7: Especifique $\hbar, \xi_{1}$ e $\xi_{2}$;

while $t_{k m}^{k}$ não satisfizer as condições $\left\|t_{k m}^{(k)}-t_{k m}^{\prime}\right\|_{\infty} \leq \xi_{1}$ ou $\left\|t_{k m}^{(k)}-t_{k m}^{(k-1)}\right\|_{\infty}<\xi_{2} \quad e$ $b_{k}^{k}$ não satisfizer as condições $\left\|b_{k}^{s h^{(k)}}-b_{k}^{\prime}\right\|_{\infty} \leq \xi_{1}$ ou $\left\|b_{k}^{s h^{(k)}}-b_{k}^{s h^{(k-1)}}\right\|_{\infty}<\xi_{2}$, onde $t_{k m}^{\prime}$ e $b_{k}^{\prime}$ são os valores discretos mais próximos de $t_{k m}^{(k)}$ e $b_{k}^{s h^{(k)}}$, respectivamente. do

Resolva o problema (5.14), com $\varpi^{k}$ fixo;

Admita $\left(V_{k}^{(k)}, t_{k m}^{(k)}, b_{k}^{s h^{(k)}}\right)$ como uma solução;

Atualize o parâmetro $\varpi$ por (5.16);

$k=k+1$;

Faça $\chi\left(y_{1_{k}}\right)$ assumir valores da seguinte forma

$$
\chi\left(y_{1_{k}}\right)^{k}= \begin{cases}0 & \text { se }\left\|V_{k}^{(k)}-A u x V_{k}^{(0)}\right\|_{\infty} \leq \xi_{5} \\ 1 & \text { c.c. }\end{cases}
$$

faça $\chi\left(y_{2_{k m}}\right)$ assumir valores da seguinte forma

$$
\chi\left(y_{2_{k m}}\right)^{k}= \begin{cases}0 & \text { se }\left\|t_{k m}^{(k)}-A u x t_{k m}^{(0)}\right\|_{\infty} \leq \xi_{5} \\ 1 & \text { c.c. }\end{cases}
$$

e faça $\chi\left(y_{3_{k}}\right)$ assumir valores da seguinte forma

$$
\chi\left(y_{3_{k}}\right)^{k}= \begin{cases}0 & \text { se }\left\|b_{k}^{s h^{(k)}}-A u x b_{k}^{(0)}\right\|_{\infty} \leq \xi_{5} \\ 1 & \text { c.c. }\end{cases}
$$


problema em questão, porém não se pode-se afirmar que esta solução é uma solução ótima local, devido à segunda fase de resolução, pois resolve-se um problema modificado.

\subsubsection{Considerações Finais}

Apresentamos neste capítulo, modelos e algoritmos para a resolução de problemas de FPO com limitação do número de ações de controles e para a resolução de problemas de FPO multiobjetivo. Este capítulo foi subdividido em quatro seções, as quais apresentam:

- Na Seção 5.1 apresenta-se o modelo para o problema de FPO com Limitação do Número de Ações de Controles (2.8) modificado pela função quadrática para o tratamento das variáveis binárias (associadas as ações de controles) e pela função de discretização polinomial (para o tratamento dos taps de transformadores);

- A Seção 5.2 é subdividida em duas partes. Na primeira parte, o problema de FPO Multiobjetivo com taps de transformadores contínuos e elementos shunts fixos é modificado pelo método da soma ponderada que transforma este problema em um problema mono-objetivo, e a função sigmoidal é utilizada para o tratamento das variáveis binárias. Apresentamos também nesta primeira parte um algoritmo para a resolução deste problema modificado. Na segunda parte, o problema de FPO Multiobjetivo com taps de transformadores contínuos e elementos shunts fixos é modificado pelo método $\epsilon$-restrito e as variáveis binárias são tratadas pela função quadrática.

- Na Seção 5.3 o problema de FPO Multiobjetivo com taps de transformadores discretos e elementos shunts fixos é modificado pelo método $\epsilon$-restrito. As variáveis binárias do problema são tratadas por uma função quadrática e as variáveis discretas pela função de discretização polinomial.

- Por fim, na última seção, o problema de FPO Multiobjetivo com taps de transformadores e elementos shunts discretos é modificado pelo método da soma ponderada. Um algoritmo é proposto para determinar as ações de controles utilizando a função sigmoidal para o tratamento das variáveis binárias. Um segundo algoritmo multiestágio é proposto englobando a discretização das variáveis taps e shunts, utilizando as funções de discretização Senoidal, Polinomial ou Mista.

O próximo Capítulo apresenta os resultados encontrados com as estratégias descritas ao longo deste documento e está divida em três subseções. Na primeira subseção, apresentaremos os resultados encontrados utilizando as funções de discretização descritas no Capítulo 3 para o tratamento dos taps e elementos shunts do problema de FPO. Na segunda subseção, apresentaremos os resultados encontrados para o problema de FPO com Limitação do Número de Ações de Controles. Finalmente, na ultima subseção apresentaremos os resultados obtidos para o problema de FPO Multiobjetivo. A segunda e a terceira subseção dos resultados apresentam os resultados numéricos para os modelos e algoritmos apresentados neste Capítulo. 


\section{Capítulo 6}

\section{Resultados Numéricos}

Este capítulo contém os resultados obtidos utilizando as estratégias desenvolvidas para o tratamento das variáveis discretas do problema de FPO, e resultados com as estratégias propostas para a resolução do problema de FPO multiobjetivo com variáveis binárias associadas às variáveis de controles.

Para avaliar a eficiência das estratégias foram realizados testes numéricos com os sistemas elétricos IEEE de 14, 30, 57, 118 e 300 barras. Os dados destes sistemas foram obtidos na página http://www.ee.washington.edu/research/pstca (acessado em dezembro de 2012). Os modelos foram implementados em GAMS (http://www.gams.com). Foram utilizados quatro pacotes de otimização na resolução dos problemas apresentados. São eles (Anexo A):

- CONOPT - é um solver comercial para problemas de otimização não linear de grande escala, baseado no método Gradiente Reduzido Generalizado (GRG) que foi proposto por (ABADIE; CARPENTIER, 1969);

- IPOPT - Interior Point Optimizer é um solver de código aberto para problemas PNL de grande escala e é uma implementação de um Algoritmo de Pontos Interiores com Filtro para modelos de programação não linear, onde as funções podem ser não convexas, entretanto, duplamente diferenciáveis;

- KNITRO - é um solver comercial para otimização não linear possuindo três algoritmos implementados: Algoritmo de Pontos Interiores Direto; Algoritmo de Pontos Interiores com Gradientes Conjugados; e Algoritmo de Conjuntos Ativos;

- DICOPT - DIscrete and Continuous OPTimizer é um solver para resolver problemas de PNLIM que baseia-se nas extensões do algoritmo outer-approximation para a estratégia de relaxamento das restrições de igualdade.

Para todos os testes realizados, consideramos que os taps de transformadores possuem limites mínimo e máximo em, 0.88 p.u. e 1.12 p.u., respectivamente. Quando estes forem 
considerados discretos, devem pertencer ao conjunto discreto igualmente espaçado por passos de 0.0075 p.u. entre 0.88 p.u. e 1.12 p.u. (33 posições). Consideramos para todos os algoritmos que a tensão tem limites inferior e superior em 0.9 p.u. e 1.1 p.u.; e a precisão adotada para as tolerâncias $\xi_{1}, \xi_{2}$ e $\xi_{3}$ foi de $10^{-4}$. As tabelas contendo os resultados foram geradas a partir do melhor resultado encontrado em dez tentativas. O número de iterações exposto está relacionado a um contador interno dos algoritmos propostos e não ao número de interação dos solvers utilizados.

Todos os testes da Seção 6.1 foram realizados em um microcomputador Intel Core 2 Quad 2.83 GHz com 4.00 GB de memória RAM, enquanto que os testes das demais seções foram realizados em um microcomputador i7-4770@3, 4 GHz com 8.00 GB de memória RAM, ambos do Laboratório de Análise de Sistemas de Energia Elétrica (LASEE) no departamento de Engenharia Elétrica da Escola de Engenharia de São Carlos (EESC) da Universidade de São Paulo (USP).

A próxima seção contém os resultados numéricos obtidos utilizando as funções de discretização Senoidal, Polinomial e Mista aplicadas aos sistemas elétricos do IEEE com três senários de carregamento. Análise dos resultados e conclusões. 


\subsection{Funções de discretização}

Nesta seção, apresentamos os resultados obtidos utilizando os Algoritmos 1, 2 e 3, abordados no Capítulo 3, na resolução dos sistemas elétricos IEEE de 14, 30, 57, 118 e 300 barras. Lembrando que o Algoritmo 1 utiliza uma função senoidal para o tratamento das variáveis discretas, o Algoritmo 2 utiliza uma função polinomial, e o Algoritmo 3 utiliza ambas as funções senoidal e polinomial. A relaxação contínua (2.6) é apresentada como um limitante inferior para as perdas ativas na transmissão do problema discreto (2.5). Todos os testes realizados nesta seção foram realizados utilizando o solver CONOPT.

Três cenários de carregamento foram considerados para cada sistema: cargas leves, médias e pesadas. A fim de gerar os diferentes níveis de carregamento, em primeiro lugar, o caso base do banco de dados especificado em sistema teste foi considerado como sendo carga leve. Em seguida, a carga do sistema foi aumentada até que a magnitude da tensão em uma barra de carga atingiu seu limite inferior, determinando assim a carga pesada. Com a carga pesada, o carregamento médio foi considerado como a carga correspondente entre as cargas leves e pesadas. Também é importante ressaltar que barras de folga foram distribuídas considerando o aumento de geração, e todas as cargas foram aumentadas com um fator de potência constante.

A Tabela 6.1 mostra as características de cada sistema para o modelo de FPO com variáveis contínuas e discretas (2.5).

Tabela 6.1 - Características dos Sistemas Elétricos

\begin{tabular}{c|ccc}
\hline $\begin{array}{c}\text { Sistema } \\
\text { Elétrico }\end{array}$ & $\begin{array}{c}\text { Restrições de } \\
\text { Igualdade }\end{array}$ & $\begin{array}{c}\text { Variáveis } \\
\text { Contínuas }\end{array}$ & $\begin{array}{c}\text { Variáveis } \\
\text { Discretas }\end{array}$ \\
\hline 14 & 22 & 27 & 4 \\
30 & 53 & 59 & 6 \\
57 & 106 & 113 & 18 \\
118 & 181 & 235 & 23 \\
300 & 530 & 599 & 121 \\
\hline
\end{tabular}

O número de iterações apresentado nas tabelas com os resultados representa um contador interno dos algoritmos de discretização propostos, não estando relacionados com o número de iterações do solver escolhido.

\subsubsection{Sistema Elétrico IEEE de 14 Barras}

O sistema elétrico IEEE de 14 barras possui as seguintes características: 1 barra de geração (slack); 4 barras com controle de geração de reativos; 9 barras de carga; 1 banco de capacitores; 17 linhas de transmissão; e 3 transformadores com tap variável.

Adota-se que a variável shunt, associada ao banco de capacitores, possui respectivamente limites mínimo e máximo em, 0.0 p.u. e 0.39 p.u. para a relaxação contínua, e deve assumir valores discretos no conjunto $\{0,0.19,0.34,0.39\}$ para o caso em que a variável shunt é considerada discreta. 
Determina-se a Relaxação Contínua do problema de FPO para o sistema IEEE de 14 barras utilizando o modelo (2.6), a Tabela 6.2 apresenta estes resultados obtidos para diferentes níveis de carga.

Tabela 6.2 - Solução da relaxação contínua para as variáveis de controle do sistema IEEE de 14 barras obtidas utilizando o modelo (2.6).

\begin{tabular}{|c|c|c|c|c|c|c|c|}
\hline \multirow{2}{*}{\multicolumn{2}{|c|}{ Barra (k) }} & \multicolumn{2}{|c|}{ Carga Leve } & \multicolumn{2}{|c|}{ Carga Média } & \multicolumn{2}{|c|}{ Carga Pesada } \\
\hline & & $\begin{array}{c}V_{k} \\
\text { (p.u.) }\end{array}$ & $\begin{array}{c}b_{k}^{s h} \\
\text { (p.u.) }\end{array}$ & $\begin{array}{c}V_{k} \\
\text { (p.u.) }\end{array}$ & $\begin{array}{c}b_{k}^{s h} \\
\text { (p.u.) }\end{array}$ & $\begin{array}{c}V_{k} \\
\text { (p.u.) }\end{array}$ & $\begin{array}{c}b_{k}^{s h} \\
\text { (p.u.) }\end{array}$ \\
\hline \multicolumn{2}{|c|}{1} & 1.1000 & - & 1.1000 & - & 1.1000 & - \\
\hline \multicolumn{2}{|c|}{2} & 1.0858 & - & 1.0847 & - & 1.0580 & - \\
\hline \multicolumn{2}{|c|}{3} & 1.0566 & 一 & 1.0515 & - & 1.0002 & 一 \\
\hline \multicolumn{2}{|c|}{6} & 1.1000 & - & 1.1000 & - & 1.0890 & - \\
\hline \multicolumn{2}{|c|}{8} & 1.0903 & - & 1.1000 & - & 1.1000 & 一 \\
\hline \multicolumn{2}{|c|}{9} & - & 0.3882 & - & 0.3900 & - & 0.3900 \\
\hline $\begin{array}{c}\text { Da barra } \\
(\mathrm{k})\end{array}$ & $\begin{array}{c}\text { Para barra } \\
(\mathrm{m})\end{array}$ & \multicolumn{2}{|c|}{$\begin{array}{c}t_{k m} \\
\text { (p.u.) }\end{array}$} & \multicolumn{2}{|c|}{$\begin{array}{c}t_{k m} \\
\text { (p.u.) }\end{array}$} & \multicolumn{2}{|c|}{$\begin{array}{c}t_{k m} \\
\text { (p.u.) }\end{array}$} \\
\hline 4 & 7 & \multicolumn{2}{|c|}{0.9504} & \multicolumn{2}{|c|}{1.0940} & \multicolumn{2}{|c|}{1.0174} \\
\hline 4 & 9 & \multicolumn{2}{|c|}{1.0804} & \multicolumn{2}{|c|}{0.8800} & \multicolumn{2}{|c|}{0.9078} \\
\hline 5 & 6 & \multicolumn{2}{|c|}{0.9821} & \multicolumn{2}{|c|}{0.9636} & \multicolumn{2}{|c|}{0.9155} \\
\hline \multicolumn{2}{|c|}{ Perdas (MW) } & \multicolumn{2}{|c|}{12.27} & \multicolumn{2}{|c|}{19.85} & \multicolumn{2}{|c|}{30.78} \\
\hline
\end{tabular}

As Tabelas 6.3, 6.4 e 6.5 apresentam os valores das variáveis de controle determinados pela resolução do modelo (2.5) para o sistema IEEE de 14 barras através dos Algoritmos 1, 2 e 3.

Tabela 6.3 - Solução do modelo (2.5) para o sistema IEEE de 14 barras pelo Algoritmo 1 (função senoidal).

\begin{tabular}{|c|c|c|c|c|c|c|c|}
\hline \multirow{2}{*}{\multicolumn{2}{|c|}{ Barra (k) }} & \multicolumn{2}{|c|}{ Carga Leve } & \multicolumn{2}{|c|}{ Carga Média } & \multicolumn{2}{|c|}{ Carga Pesada } \\
\hline & & \multicolumn{2}{|c|}{ 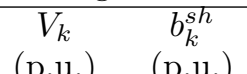 } & \multicolumn{2}{|c|}{$\begin{array}{cc}V_{k} & b_{k}^{s h} \\
( & \end{array}$} & \multicolumn{2}{|c|}{$\begin{array}{cc}V_{k} & b_{k}^{s h} \\
\text { (p.u.) } & \text { (p.u.) } \\
\end{array}$} \\
\hline \multicolumn{2}{|c|}{1} & 1.1000 & - & 1.1000 & - & 1.1000 & $=$ \\
\hline \multicolumn{2}{|c|}{2} & 1.0862 & - & 1.0843 & - & 1.0579 & - \\
\hline \multicolumn{2}{|c|}{3} & 1.0573 & - & 1.0500 & - & 1.0001 & - \\
\hline \multicolumn{2}{|c|}{6} & 1.0962 & - & 1.1000 & - & 1.0873 & - \\
\hline \multicolumn{2}{|c|}{8} & 1.0950 & - & 1.0999 & - & 1.1000 & - \\
\hline \multicolumn{2}{|c|}{9} & - & 0.3900 & - & 0.3900 & - & 0.3900 \\
\hline $\begin{array}{c}\text { Da barra } \\
(\mathrm{k})\end{array}$ & $\begin{array}{c}\text { Para barra } \\
(\mathrm{m})\end{array}$ & \multicolumn{2}{|c|}{$\begin{array}{c}t_{k m} \\
\text { (p.u.) }\end{array}$} & \multicolumn{2}{|c|}{$\begin{array}{l}t_{k m} \\
\text { (p.u.) }\end{array}$} & \multicolumn{2}{|c|}{$\begin{array}{l}t_{k m} \\
\text { (p.u.) }\end{array}$} \\
\hline 4 & 7 & \multicolumn{2}{|c|}{0.9475} & \multicolumn{2}{|c|}{1.0975} & \multicolumn{2}{|c|}{1.0151} \\
\hline 4 & 9 & \multicolumn{2}{|c|}{1.1200} & \multicolumn{2}{|c|}{0.8800} & \multicolumn{2}{|c|}{0.9100} \\
\hline 5 & 6 & \multicolumn{2}{|c|}{0.9850} & \multicolumn{2}{|c|}{0.9625} & \multicolumn{2}{|c|}{0.9175} \\
\hline \multirow{2}{*}{\multicolumn{2}{|c|}{$\gamma_{0}$}} & \multicolumn{2}{|c|}{0.0001} & \multicolumn{2}{|c|}{0.0001} & \multicolumn{2}{|c|}{0.0001} \\
\hline & & \multirow{2}{*}{\multicolumn{2}{|c|}{$\begin{array}{c}10 \\
0.625\end{array}$}} & \multirow{2}{*}{\multicolumn{2}{|c|}{$\begin{array}{c}10 \\
0.469\end{array}$}} & \multirow{2}{*}{\multicolumn{2}{|c|}{$\begin{array}{c}10 \\
0.625\end{array}$}} \\
\hline \multicolumn{2}{|c|}{$\begin{array}{c}c \\
\text { Tempo }(\mathrm{s})\end{array}$} & & & & & & \\
\hline \multirow{2}{*}{\multicolumn{2}{|c|}{$\begin{array}{l}\text { Número de iterações } \\
\text { Perdas (MW) }\end{array}$}} & \multicolumn{2}{|c|}{2} & \multicolumn{2}{|c|}{2} & \multicolumn{2}{|c|}{3} \\
\hline & & & & & & & \\
\hline
\end{tabular}


Tabela 6.4 - Solução do modelo (2.5) para o sistema IEEE de 14 barras pelo Algoritmo 2 (função polinomial).

\begin{tabular}{|c|c|c|c|c|c|c|c|}
\hline \multirow{2}{*}{\multicolumn{2}{|c|}{ Barra (k) }} & \multicolumn{2}{|c|}{ Carga Leve } & \multicolumn{2}{|c|}{ Carga Média } & \multicolumn{2}{|c|}{ Carga Pesada } \\
\hline & & $\begin{array}{c}V_{k} \\
\text { (p.u.) }\end{array}$ & $\begin{array}{c}b_{k}^{s h} \\
\text { (p.u.) }\end{array}$ & $\begin{array}{c}V_{k} \\
\text { (p.u.) }\end{array}$ & $\begin{array}{c}b_{k}^{s h} \\
\text { (p.u.) }\end{array}$ & $\begin{array}{c}V_{k} \\
\text { (p.u.) }\end{array}$ & $\begin{array}{c}b_{k}^{s h} \\
\text { (p.u.) }\end{array}$ \\
\hline \multicolumn{2}{|c|}{1} & 1.1000 & - & 1.1000 & - & 1.1000 & - \\
\hline \multicolumn{2}{|c|}{2} & 1.0865 & - & 1.0843 & - & 1.0579 & - \\
\hline \multicolumn{2}{|c|}{3} & 1.0578 & - & 1.0500 & - & 1.0001 & - \\
\hline \multicolumn{2}{|c|}{6} & 1.0990 & - & 1.1000 & - & 1.0873 & - \\
\hline \multicolumn{2}{|c|}{8} & 1.0936 & - & 1.0998 & - & 1.1000 & - \\
\hline \multicolumn{2}{|c|}{9} & - & 0.3900 & - & 0.3900 & - & 0.3900 \\
\hline $\begin{array}{c}\text { Da barra } \\
(\mathrm{k})\end{array}$ & $\begin{array}{c}\text { Para barra } \\
(\mathrm{m})\end{array}$ & \multicolumn{2}{|c|}{$\begin{array}{c}t_{k m} \\
\text { (p.u.) }\end{array}$} & \multicolumn{2}{|c|}{$\begin{array}{l}t_{k m} \\
\text { (p.u.) }\end{array}$} & \multicolumn{2}{|c|}{$\begin{array}{c}t_{k m} \\
\text { (p.u.) }\end{array}$} \\
\hline 4 & 7 & \multicolumn{2}{|c|}{0.9550} & \multicolumn{2}{|c|}{1.0975} & \multicolumn{2}{|c|}{1.0150} \\
\hline 4 & 9 & \multirow{2}{*}{\multicolumn{2}{|c|}{$\begin{array}{l}1.0825 \\
0.9850\end{array}$}} & \multirow{2}{*}{\multicolumn{2}{|c|}{$\begin{array}{l}0.8800 \\
0.9625\end{array}$}} & \multirow{2}{*}{\multicolumn{2}{|c|}{$\begin{array}{l}0.9100 \\
0.9175\end{array}$}} \\
\hline 5 & 6 & & & & & & \\
\hline \multicolumn{2}{|c|}{$\overline{\nu_{0}}$} & \multicolumn{2}{|c|}{0.000953} & \multicolumn{2}{|c|}{0.000953} & \multicolumn{2}{|c|}{0.000953} \\
\hline \multirow{2}{*}{\multicolumn{2}{|c|}{$\begin{array}{c}c_{1} \\
\text { Tempo (s) }\end{array}$}} & \multirow{2}{*}{\multicolumn{2}{|c|}{$\begin{array}{c}4.5 \\
0.703\end{array}$}} & \multicolumn{2}{|c|}{$\begin{array}{c}4.5 \\
1047\end{array}$} & \multirow{2}{*}{\multicolumn{2}{|c|}{$\begin{array}{c}4.5 \\
0.469\end{array}$}} \\
\hline & & & & \multirow{2}{*}{\multicolumn{2}{|c|}{2}} & & \\
\hline \multicolumn{2}{|c|}{ Número de iterações } & \multicolumn{2}{|c|}{2} & & & \multicolumn{2}{|c|}{2} \\
\hline \multicolumn{2}{|c|}{ Perdas (MW) } & \multicolumn{2}{|c|}{12.27} & \multicolumn{2}{|c|}{19.85} & \multicolumn{2}{|c|}{30.78} \\
\hline
\end{tabular}

Tabela 6.5 - Solução do modelo (2.5) para o sistema IEEE de 14 barras pelo Algoritmo 3 (funções senoidal e polinomial).

\begin{tabular}{|c|c|c|c|c|c|c|c|}
\hline \multirow{2}{*}{\multicolumn{2}{|c|}{ Barra (k) }} & \multicolumn{2}{|c|}{ Carga Leve } & \multicolumn{2}{|c|}{ Carga Média } & \multicolumn{2}{|c|}{ Carga Pesada } \\
\hline & & $\begin{array}{c}V_{k} \\
\text { (p.u.) }\end{array}$ & $\begin{array}{c}b_{k}^{s h} \\
\text { (p.u.) }\end{array}$ & $\begin{array}{c}V_{k} \\
\text { (p.u.) }\end{array}$ & $\begin{array}{c}b_{k}^{s h} \\
\text { (p.u.) }\end{array}$ & $\begin{array}{c}V_{k} \\
\text { (p.u.) }\end{array}$ & $\begin{array}{c}b_{k}^{s h} \\
\text { (p.u.) }\end{array}$ \\
\hline \multicolumn{2}{|c|}{1} & 1.1000 & - & 1.1000 & - & 1.1000 & - \\
\hline \multicolumn{2}{|c|}{2} & 1.0847 & - & 1.0843 & - & 1.0579 & - \\
\hline \multicolumn{2}{|c|}{3} & 1.0545 & - & 1.0500 & - & 1.0001 & - \\
\hline \multicolumn{2}{|c|}{6} & 1.0972 & - & 1.1000 & - & 1.0873 & - \\
\hline \multicolumn{2}{|c|}{8} & 1.0903 & - & 1.0999 & - & 1.1000 & - \\
\hline \multicolumn{2}{|c|}{9} & - & 0.3900 & - & 0.3900 & - & 0.3900 \\
\hline $\begin{array}{c}\text { Da barra } \\
(\mathrm{k})\end{array}$ & $\begin{array}{l}\text { Para barra } \\
(\mathrm{m})\end{array}$ & \multicolumn{2}{|c|}{$\begin{array}{c}t_{k m} \\
\text { (p.u.) }\end{array}$} & \multicolumn{2}{|c|}{$\begin{array}{c}t_{k m} \\
\text { (p.u.) }\end{array}$} & \multicolumn{2}{|c|}{$\begin{array}{c}t_{k m} \\
\text { (p.u.) }\end{array}$} \\
\hline 4 & 7 & \multicolumn{2}{|c|}{0.9476} & \multicolumn{2}{|c|}{1.0975} & \multicolumn{2}{|c|}{1.0151} \\
\hline 4 & 9 & \multicolumn{2}{|c|}{1.0825} & \multicolumn{2}{|c|}{0.8800} & \multicolumn{2}{|c|}{0.9100} \\
\hline 5 & 6 & \multicolumn{2}{|c|}{0.9850} & \multicolumn{2}{|c|}{0.9625} & \multicolumn{2}{|c|}{0.9175} \\
\hline \multicolumn{2}{|c|}{$\gamma_{0}$} & \multicolumn{2}{|c|}{0.0001} & \multicolumn{2}{|c|}{0.0001} & \multicolumn{2}{|c|}{0.0001} \\
\hline \multicolumn{2}{|c|}{$\begin{array}{c}c \\
\nu_{0}\end{array}$} & \multicolumn{2}{|c|}{10} & \multirow{2}{*}{\multicolumn{2}{|c|}{$\begin{array}{c}10 \\
0.000953\end{array}$}} & \multicolumn{2}{|c|}{10} \\
\hline \multirow{2}{*}{\multicolumn{2}{|c|}{$\begin{array}{l}\nu_{0} \\
c_{1}\end{array}$}} & \multicolumn{2}{|c|}{0.000953} & & & 0.0 & \\
\hline & & \multirow{2}{*}{\multicolumn{2}{|c|}{$\begin{array}{c}4.5 \\
0.437\end{array}$}} & \multicolumn{2}{|c|}{4.5} & \multicolumn{2}{|c|}{4.5} \\
\hline \multicolumn{2}{|c|}{$\begin{array}{c}c_{1} \\
\text { Tempo (s) }\end{array}$} & & & & & & \\
\hline Número & e iterações & & & & & & \\
\hline Perd & (MW) & & & & & & \\
\hline
\end{tabular}




\subsubsection{Sistema Elétrico IEEE de 30 Barras}

O sistema elétrico IEEE 30 de barras possui as seguintes características: 1 barra de geração (slack); 5 barras com controle de geração de reativos; 24 barras de carga; 2 bancos de capacitores; 37 linhas de transmissão; e 4 transformadores com tap variável.

Na relaxação contínua a variável associada ao banco de capacitores da barra 10 possui limites mínimo e máximo em, 0.0 p.u. e 0.39 p.u., respectivamente; quando considerada discreta deve assumir valores discretos no conjunto $\{0,0.19,0.34,0.39\}$. A variável associada ao banco de capacitores da barra 24, possui limites mínimo e máximo em, 0.0 p.u. e 0.09 p.u., respectivamente, para a relaxação contínua; no caso discreto deve assumir valores discretos no conjunto $\{0,0.05,0.09\}$.

Determina-se a Relaxação Contínua do problema de FPO para o sistema IEEE de 30 barras utilizando o modelo (2.6), a Tabela 6.6 apresenta esses resultados obtidos para diferentes níveis de carga.

Tabela 6.6 - Solução da relaxação contínua para as variáveis de controle do sistema IEEE de 30 barras obtidos utilizando o modelo (2.6).

\begin{tabular}{c|c|c|c|c}
\hline \multicolumn{2}{l|}{} & Carga Leve & Carga Média & Carga Pesada \\
\hline Shunt & 10 & 0.3280 & 0.3900 & 0.3900 \\
Barra (k) & 24 & 0.0900 & 0.0900 & 0.0900 \\
\hline \multirow{3}{*}{ Tap } & $6-9$ & 1.0621 & 1.0902 & 1.0421 \\
Ramo (k-m) & $6-10$ & 0.9640 & 0.9572 & 0.9650 \\
& $4-12$ & 0.9796 & 0.9890 & 0.9723 \\
& $28-27$ & 0.9580 & 0.9545 & 0.9306 \\
\hline \multicolumn{2}{c|}{ Perdas (MW) } & 15.98 & 21.12 & 27.55 \\
\hline
\end{tabular}

A Tabela 6.7 apresenta os valores das variáveis de controle discretas determinadas na resolução do modelo (2.5) pelos Algoritmo 1, 2 e 3. 
Tabela 6.7 - Solução do modelo (2.5) para o sistema IEEE de 30 barras pelos Algoritmos 1, 2 e 3 .

\begin{tabular}{|c|c|c|c|c|}
\hline \multicolumn{5}{|c|}{ Parâmetros Iniciais } \\
\hline & & Carga Leve & Carga Média & Carga Pesada \\
\hline \multicolumn{2}{|l|}{$\gamma_{0}$} & 0.000001 & 0.000001 & 0.00001 \\
\hline \multicolumn{2}{|l|}{$c$} & 10 & 10 & 10 \\
\hline \multicolumn{2}{|l|}{$\nu_{0}$} & 0.000022 & 0.000030 & 0.0000045 \\
\hline \multicolumn{2}{|l|}{$c_{1}$} & 4.5 & 4.5 & 4.5 \\
\hline \multicolumn{5}{|c|}{ Algoritmo 1 (Senoidal) } \\
\hline \multicolumn{3}{|r|}{ Carga Leve } & \multicolumn{2}{|l|}{ Carga M } \\
\hline Shunt & 10 & 0.3399 & 0.3900 & 0.3900 \\
\hline Barra (k) & 24 & 0.0900 & 0.0900 & 0.0900 \\
\hline \multirow{4}{*}{$\begin{array}{c}\text { Tap } \\
\text { Ramo (k-m) }\end{array}$} & $6-9$ & 1.0600 & 1.0900 & 1.0450 \\
\hline & $6-10$ & 0.9625 & 0.9550 & 0.9625 \\
\hline & $4-12$ & 0.9775 & 0.9925 & 0.9701 \\
\hline & $28-27$ & 0.9550 & 0.9550 & 0.9325 \\
\hline \multicolumn{2}{|c|}{ Tempo (s) } & 0.656 & 0.625 & 0.984 \\
\hline \multirow{2}{*}{\multicolumn{2}{|c|}{$\begin{array}{l}\text { Número de Iterações } \\
\text { Perdas }(\mathrm{MW})\end{array}$}} & 4 & 3 & 3 \\
\hline & & 15.98 & 21.12 & 27.56 \\
\hline \multicolumn{5}{|c|}{ Algoritmo 2 (Polinomial) } \\
\hline & & Carga Leve & Carga Média & Carga Pesada \\
\hline Shunt & 10 & 0.3399 & 0.3900 & 0.3900 \\
\hline Barra (k) & 24 & 0.0900 & 0.0900 & 0.0900 \\
\hline \multirow{4}{*}{$\begin{array}{c}\text { Tap } \\
\text { Ramo (k-m) }\end{array}$} & $6-9$ & 1.0600 & 1.0900 & 1.0450 \\
\hline & $6-10$ & 0.9625 & 0.9550 & 0.9625 \\
\hline & $4-12$ & 0.9775 & 0.9925 & 0.9701 \\
\hline & $28-27$ & 0.9550 & 0.9550 & 0.9325 \\
\hline \multirow{3}{*}{\multicolumn{2}{|c|}{$\begin{array}{c}\text { Tempo }(\mathrm{s}) \\
\text { Número de Iterações } \\
\text { Perdas }(\mathrm{MW})\end{array}$}} & 0.969 & 0.766 & 0.875 \\
\hline & & 3 & 2 & 2 \\
\hline & & 15.98 & 21.12 & 27.56 \\
\hline \multicolumn{5}{|c|}{ Algoritmo 3 (Senoidal e Polinomial) } \\
\hline \multicolumn{2}{|c|}{ 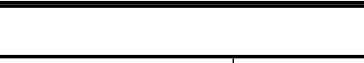 } & Carga Leve & Carga Média & Carga Pesada \\
\hline Shunt & 10 & 0.3400 & 0.3900 & 0.3900 \\
\hline Barra $(\mathrm{k})$ & 24 & 0.0900 & 0.0900 & 0.0900 \\
\hline \multirow{4}{*}{$\begin{array}{c}\text { Tap } \\
\text { Ramo (k-m) }\end{array}$} & $6-9$ & 1.0675 & 1.0900 & 1.0450 \\
\hline & $6-10$ & 0.9625 & 0.9550 & 0.9625 \\
\hline & $4-12$ & 0.9701 & 0.9925 & 0.9701 \\
\hline & $28-27$ & 0.9550 & 0.9550 & 0.9325 \\
\hline \multirow{3}{*}{\multicolumn{2}{|c|}{$\begin{array}{c}\text { Tempo (s) } \\
\text { Número de Iterações } \\
\text { Perdas }(\mathrm{MW})\end{array}$}} & 0.656 & 0.609 & 0.609 \\
\hline & & 3 & 3 & 3 \\
\hline & & 15.98 & 21.12 & 27.56 \\
\hline
\end{tabular}




\subsubsection{Sistema Elétrico IEEE de 57 Barras}

O sistema elétrico IEEE de 57 barras possui as seguintes características: 1 barra de geração (slack); 6 barras com controle de geração de reativos; 50 barras de carga; 3 bancos de capacitores; 80 linhas de transmissão; e 20 transformadores com tap variável.

A Tabela 6.8 apresenta os valores discretos que cada variável shunt pode assumir quando considerada discreta, e os limitantes assumidos para a relaxação contínua.

Tabela 6.8 - Valores mínimo, máximo e discretos das susceptâncias e reatores shunts.

\begin{tabular}{ccccc}
\hline $\begin{array}{c}\text { Variáveis } \\
b_{k}^{s h}\end{array}$ & $\begin{array}{c}\text { Barra } \\
\text { Equivalente }\end{array}$ & $\begin{array}{c}\text { Limite Min } \\
\text { (p.u.) }\end{array}$ & $\begin{array}{c}\text { Limite Max } \\
\text { (p.u.) }\end{array}$ & $\begin{array}{c}\text { Conjunto de Valores } \\
\text { Discretos de } b_{k}^{s h}\end{array}$ \\
\hline 1 & 18 & 0.000 & 0.270 & $\{0,0.12,0.22,0.2\}$ \\
2 & 25 & 0.000 & 0.090 & $\{0,0.04,0.07,0.09\}$ \\
3 & 53 & 0.000 & 0.165 & $\{0,0.10,0.165\}$ \\
\hline
\end{tabular}

A Tabela 6.9 contêm os resultados do sistema IEEE de 57 barras considerando a Relaxação Contínua (2.6) e o modelo discreto (2.5) pelos os Algoritmos 1, 2 e 3.

Tabela 6.9 - Resultados para o sistema IEEE de 57 barras.

\begin{tabular}{|c|c|c|c|}
\hline \multicolumn{4}{|c|}{ Relaxação Contínua } \\
\hline & Carga Leve & Carga Média & Carga Pesada \\
\hline Tempo (s) & 0.359 & 0.375 & 0.313 \\
\hline Perdas (MW) & 20.92 & 22.78 & 25.09 \\
\hline \multicolumn{4}{|c|}{ Parâmetros Iniciais } \\
\hline & Carga Leve & Carga Média & Carga Pesada \\
\hline$\gamma_{0}$ & 0.000001 & 0.000001 & 0.000001 \\
\hline$c$ & 10 & 10 & 10 \\
\hline$\nu_{0}$ & 0.00042386 & 0.00442222 & 0.00046468 \\
\hline$c_{1}$ & 3.5 & 3.5 & 3.5 \\
\hline \multicolumn{4}{|c|}{ Algoritmo 1 (Senoidal) } \\
\hline & Carga Leve & Carga Média & Carga Pesada \\
\hline Tempo (s) & 2.966 & 3.063 & 2.640 \\
\hline Número de Iterações & 7 & 7 & 6 \\
\hline Perdas (MW) & 22.48 & 24.33 & 26.70 \\
\hline \multicolumn{4}{|c|}{ Algoritmo 2 (Polinomial) } \\
\hline & Carga Leve & Carga Média & Carga Pesada \\
\hline Tempo (s) & 3.047 & 3.297 & 2.703 \\
\hline Número de Iterações & 6 & 5 & 5 \\
\hline Perdas (MW) & 22.48 & 24.33 & 26.70 \\
\hline \multicolumn{4}{|c|}{ Algoritmo 3 (Senoidal e Polinomial) } \\
\hline & Carga Leve & Carga Média & Carga Pesada \\
\hline Tempo (s) & 1.766 & 2.469 & 2.187 \\
\hline Número de Iterações & 5 & 5 & 5 \\
\hline Perdas (MW) & 22.37 & 24.28 & 26.67 \\
\hline
\end{tabular}


As Figuras 6.1, 6.2 e 6.3 apresentam os valores discretos dos taps dos transformadores encontrados para o modelo (2.5) os Algoritmos 1, 2 e 3 considerando a carga pesada, respectivamente.

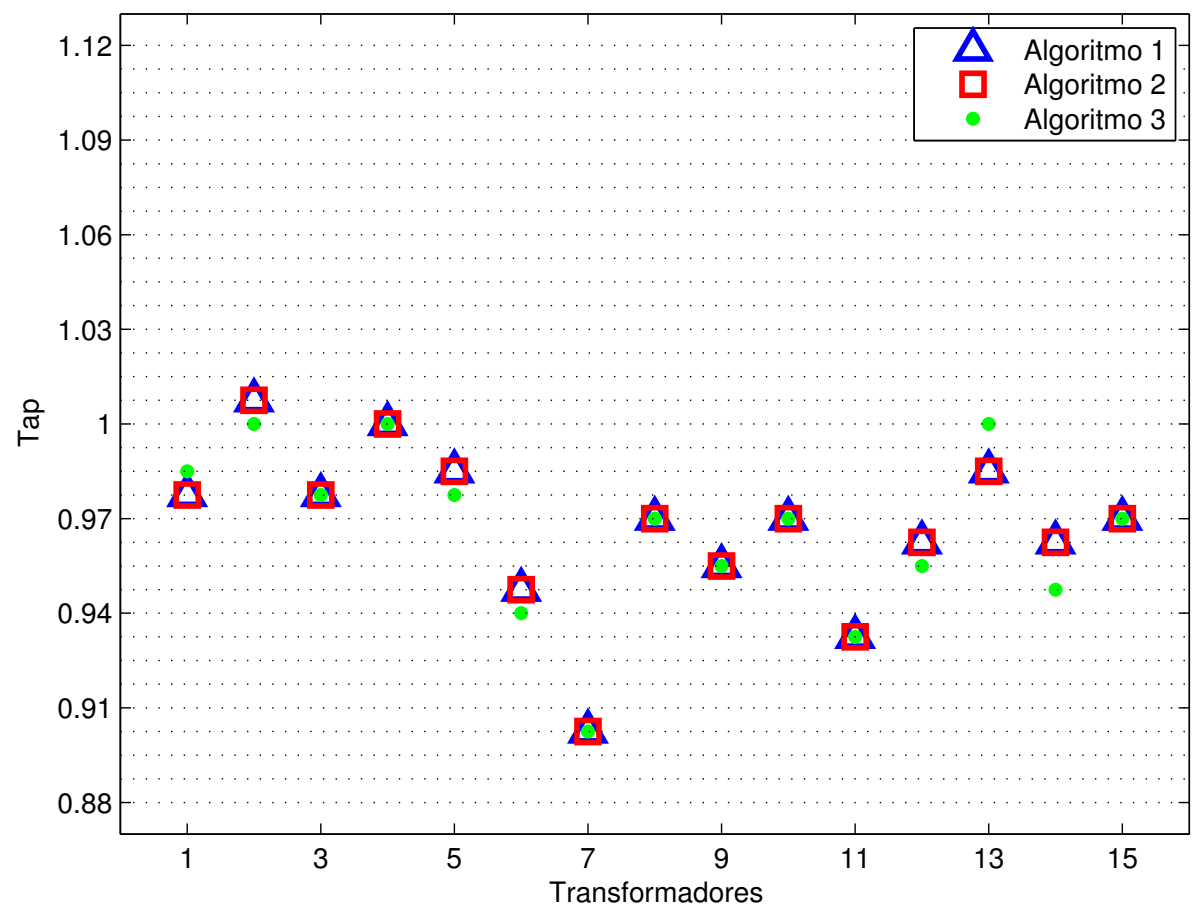

Figura 6.1 - Tap discreto para o modelo (2.5) os Algoritmos 1, 2 e 3 considerando a carga leve.

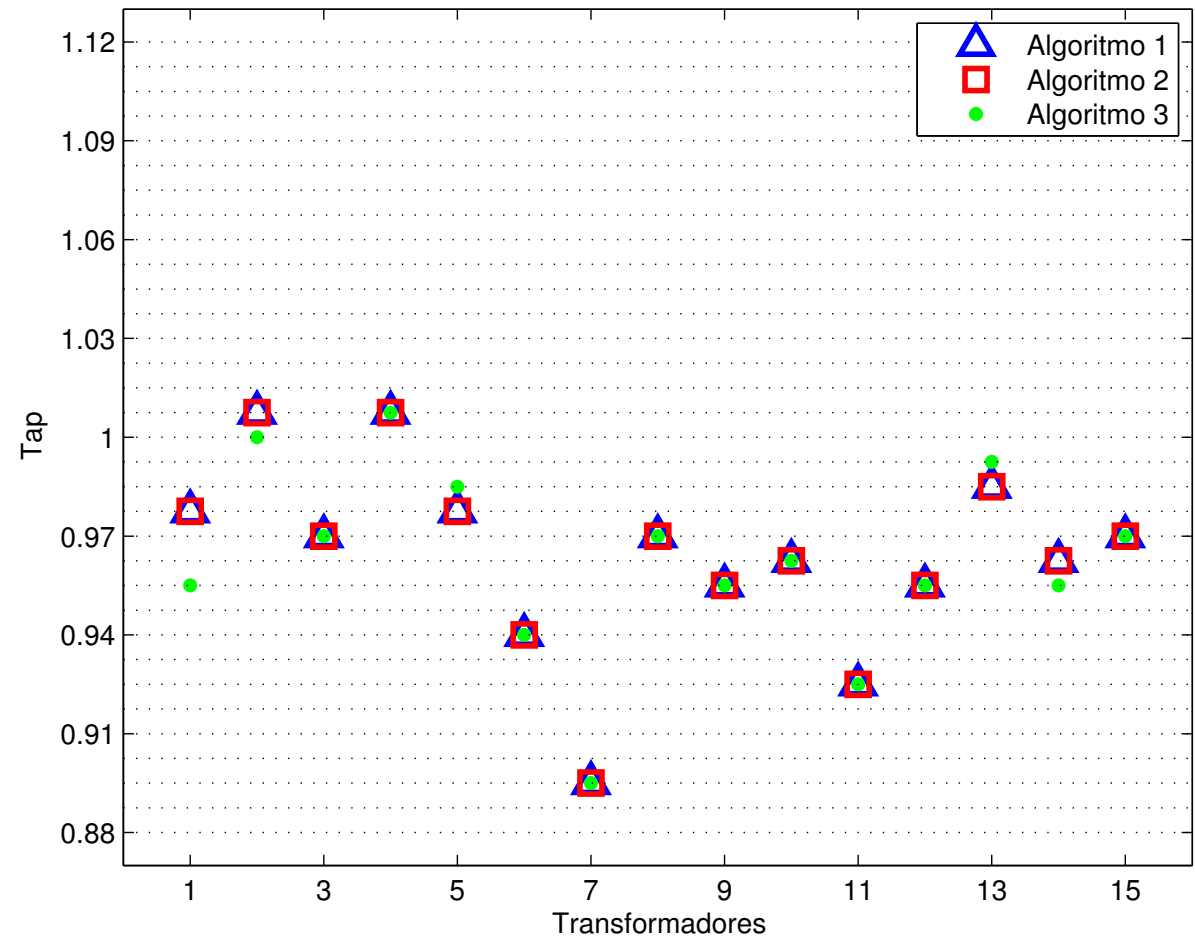

Figura 6.2 - Tap discreto para o modelo (2.5) os Algoritmos 1, 2 e 3 considerando a carga média. 


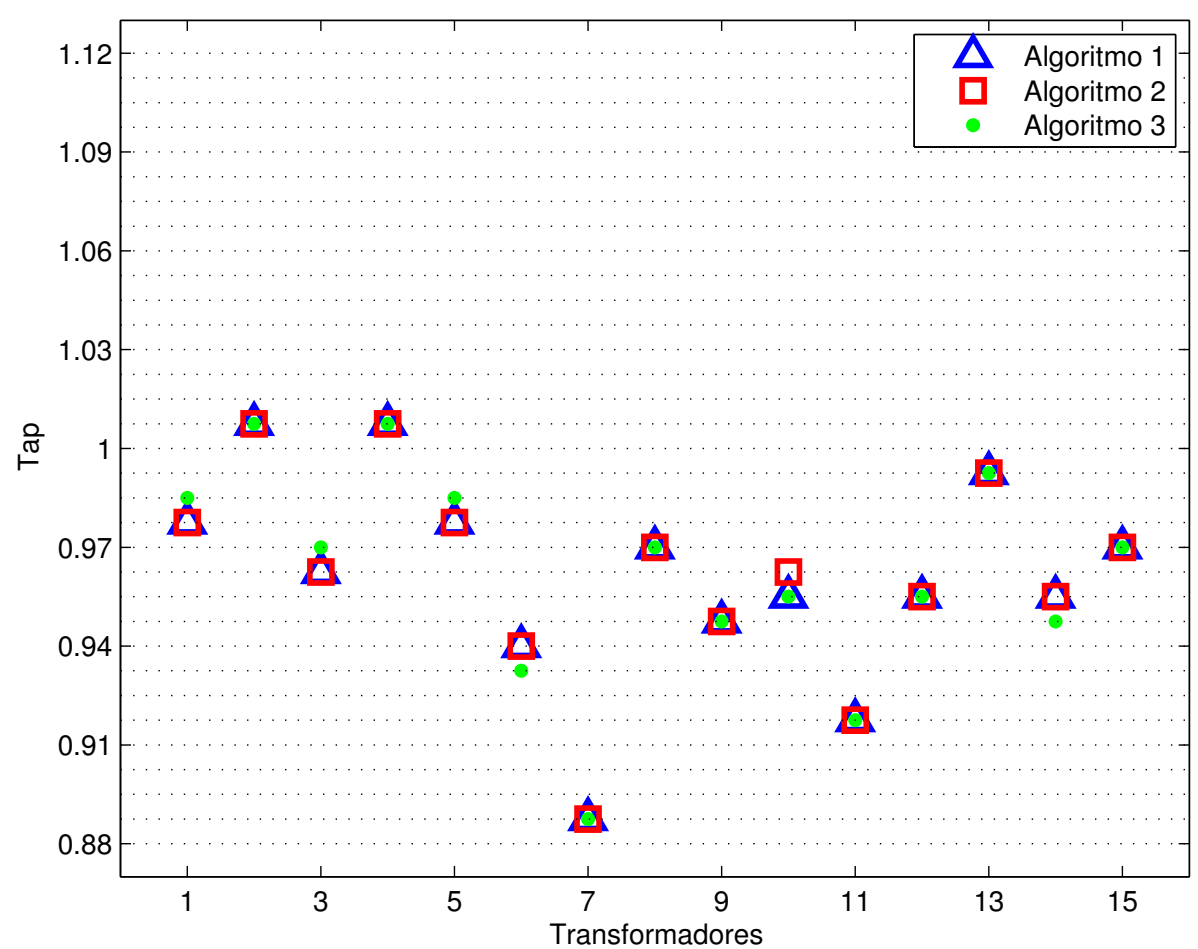

Figura 6.3 - Tap discreto para o modelo (2.5) os Algoritmos 1, 2 e 3 considerando a carga pesada.

A Tabela 6.10 apresenta os valores discretos para as susceptâncias shunts encontrados para o modelo (2.5) os Algoritmos 1, 2 e 3 considerando a carga pesada.

Tabela 6.10 - Shunt discreto encontrado para o modelo (2.5) com os Algoritmos 1, 2 e 3 considerando as cargas leve, média e pesada.

\begin{tabular}{|c|c|c|c|c|}
\hline \multicolumn{5}{|c|}{ Carga Leve } \\
\hline & & Algoritmo 1 & Algoritmo 2 & Algoritmo 3 \\
\hline Shunt & 18 & 0.1200 & 0.1200 & 0.1200 \\
\hline Barra (k) & 25 & 0.0900 & 0.0900 & 0.0900 \\
\hline & 53 & 0.1000 & 0.1000 & 0.1000 \\
\hline \multicolumn{5}{|c|}{ Carga Média } \\
\hline & & Algoritmo 1 & Algoritmo 2 & Algoritmo 3 \\
\hline Shunt & 18 & 0.1200 & 0.1200 & 0.0000 \\
\hline \multirow[t]{2}{*}{ Barra (k) } & 25 & 0.0900 & 0.0900 & 0.0900 \\
\hline & 53 & 0.1000 & 0.1000 & 0.1650 \\
\hline \multicolumn{5}{|c|}{ Carga Pesada } \\
\hline & & Algoritmo 1 & Algoritmo 2 & Algoritmo 3 \\
\hline Shunt & 18 & 0.1200 & 0.1200 & 0.1200 \\
\hline \multirow[t]{2}{*}{ Barra (k) } & 25 & 0.0900 & 0.0900 & 0.0900 \\
\hline & 53 & 0.1650 & 0.1650 & 0.1650 \\
\hline
\end{tabular}




\subsubsection{Sistema Elétrico IEEE de 118 Barras}

O sistema elétrico IEEE de 118 barras possui as seguintes características: 1 barra de geração (slack); 53 barras com controle de geração de reativos; 64 barras de carga; 12 bancos de capacitores; 2 reatores; 172 linhas de transmissão; e 9 transformadores com tap variável.

A Tabela 6.11 apresenta os valores discretos que cada variável shunt pode assumir quando considerada discreta, e os limitantes assumidos para a relaxação contínua.

Tabela 6.11 - Valores mínimo, máximo e discretos das susceptâncias e reatores shunts.

\begin{tabular}{ccccc}
\hline $\begin{array}{c}\text { Variáveis } \\
b_{k}^{s h}\end{array}$ & $\begin{array}{c}\text { Barra } \\
\text { Equivalente }\end{array}$ & $\begin{array}{c}\text { Limite Min } \\
\text { (p.u.) }\end{array}$ & $\begin{array}{c}\text { Limite Max } \\
\text { (p.u.) }\end{array}$ & $\begin{array}{c}\text { Conjunto de Valores } \\
\text { Discretos de } b_{k}^{s h}\end{array}$ \\
\hline 1 & 5 & -0.400 & 0.000 & $\{-0.4,0.0\}$ \\
2 & 34 & 0.000 & 0.210 & $\{0.0,0.14,0.20,0.21\}$ \\
3 & 37 & -0.250 & 0.000 & $\{-0.25,0.0\}$ \\
4 & 44 & 0.000 & 0.100 & $\{0.0,0.10\}$ \\
5 & 45 & 0.000 & 0.100 & $\{0.0,0.10\}$ \\
6 & 46 & 0.000 & 0.100 & $\{0.0,0.10\}$ \\
7 & 48 & 0.000 & 0.150 & $\{0.0,0.15\}$ \\
8 & 74 & 0.000 & 0.200 & $\{0.0,0.12,0.20\}$ \\
9 & 79 & 0.000 & 0.200 & $\{0.0,0.10,0.20\}$ \\
10 & 82 & 0.000 & 0.200 & $\{0.0,0.10,0.20\}$ \\
11 & 83 & 0.000 & 0.200 & $\{0.0,0.10,0.20\}$ \\
12 & 105 & 0.000 & 0.200 & $\{0.0,0.10,0.20\}$ \\
13 & 107 & 0.000 & 0.210 & $\{0.0,0.14,0.20,0.21\}$ \\
14 & 110 & 0.000 & 0.210 & $\{0.0,0.14,0.20,0.21\}$ \\
\hline
\end{tabular}

A Tabela 6.12 contêm os resultados do sistema IEEE de 118 barras considerando a Relaxação Contínua (2.6) e o modelo discreto (2.5) pelos os Algoritmos 1, 2 e 3. 
Tabela 6.12 - Resultados para o sistema IEEE de 118 barras.

\begin{tabular}{|c|c|c|c|}
\hline \multicolumn{4}{|c|}{ Relaxação Contínua } \\
\hline & Carga Leve & Carga Média & Carga Pesada \\
\hline Tempo (s) & 1.500 & 0.750 & 0.891 \\
\hline Perdas (MW) & 106.05 & 175.84 & 261.98 \\
\hline \multicolumn{4}{|c|}{ Parâmetros Iniciais } \\
\hline & Carga Leve & Carga Média & Carga Pesada \\
\hline$\gamma_{0}$ & 0.000001 & 0.000001 & 0.000001 \\
\hline$c$ & 10 & 10 & 10 \\
\hline$\nu_{0}$ & 0.000039 & 0.0000039 & 0.000005 \\
\hline$c_{1}$ & 4.5 & 4.5 & 4.5 \\
\hline \multicolumn{4}{|c|}{ Algoritmo 1 (Senoidal) } \\
\hline & Carga Leve & Carga Média & Carga Pesada \\
\hline Tempo (s) & 5.031 & 5.625 & 5.078 \\
\hline Número de Iterações & 5 & 6 & 5 \\
\hline Perdas (MW) & 106.11 & 175.93 & 262.01 \\
\hline \multicolumn{4}{|c|}{ Algoritmo 2 (Polinomial) } \\
\hline & Carga Leve & Carga Média & Carga Pesada \\
\hline Tempo (s) & 5.797 & 8.641 & 5.578 \\
\hline Número de Iterações & 6 & 7 & 6 \\
\hline Perdas (MW) & 106.10 & 175.93 & 262.01 \\
\hline \multicolumn{4}{|c|}{ Algoritmo 3 (Senoidal e Polinomial) } \\
\hline & Carga Leve & Carga Média & Carga Pesada \\
\hline Tempo (s) & 4.078 & 4.937 & 5.343 \\
\hline Número de Iterações & 5 & 5 & 5 \\
\hline Perdas (MW) & 106.11 & 175.92 & 262.01 \\
\hline
\end{tabular}

As Figuras 6.4 e 6.6 apresentam os resultados da Relaxação Contínua (2.6), para as variáveis de controle tap dos transformadores e susceptância e reatores shunt considerando a carga pesada.

As Figuras 6.5 e 6.7 apresentam os resultados encontrados para as variáveis de controle tap dos transformadores e susceptância e reatores shunt para o modelo (2.5) com os Algoritmos 1, 2 e 3 considerando a carga pesada. 


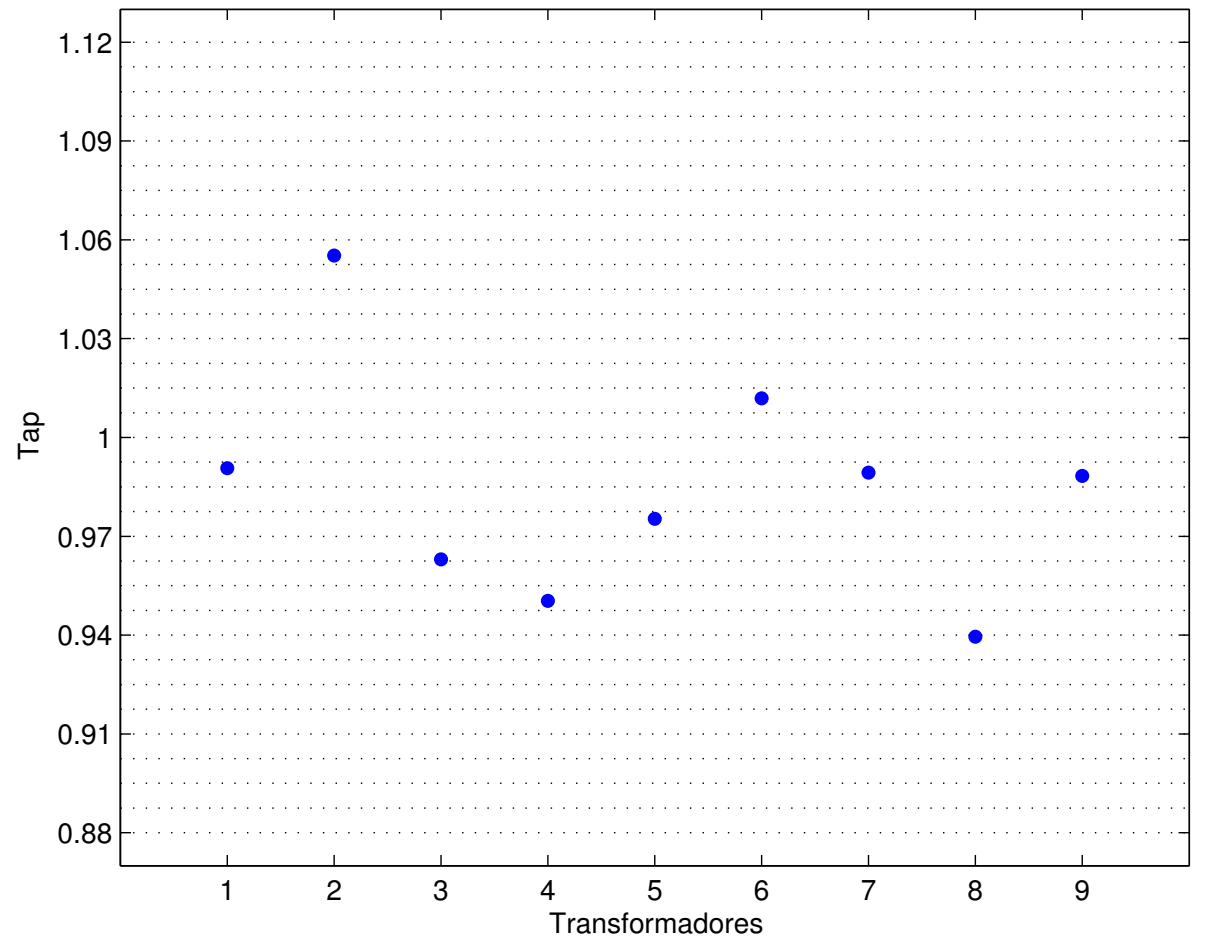

Figura 6.4 - Tap contínuo para o sistema IEEE de 118 barras pelo modelo (2.6) considerando a carga pesada.

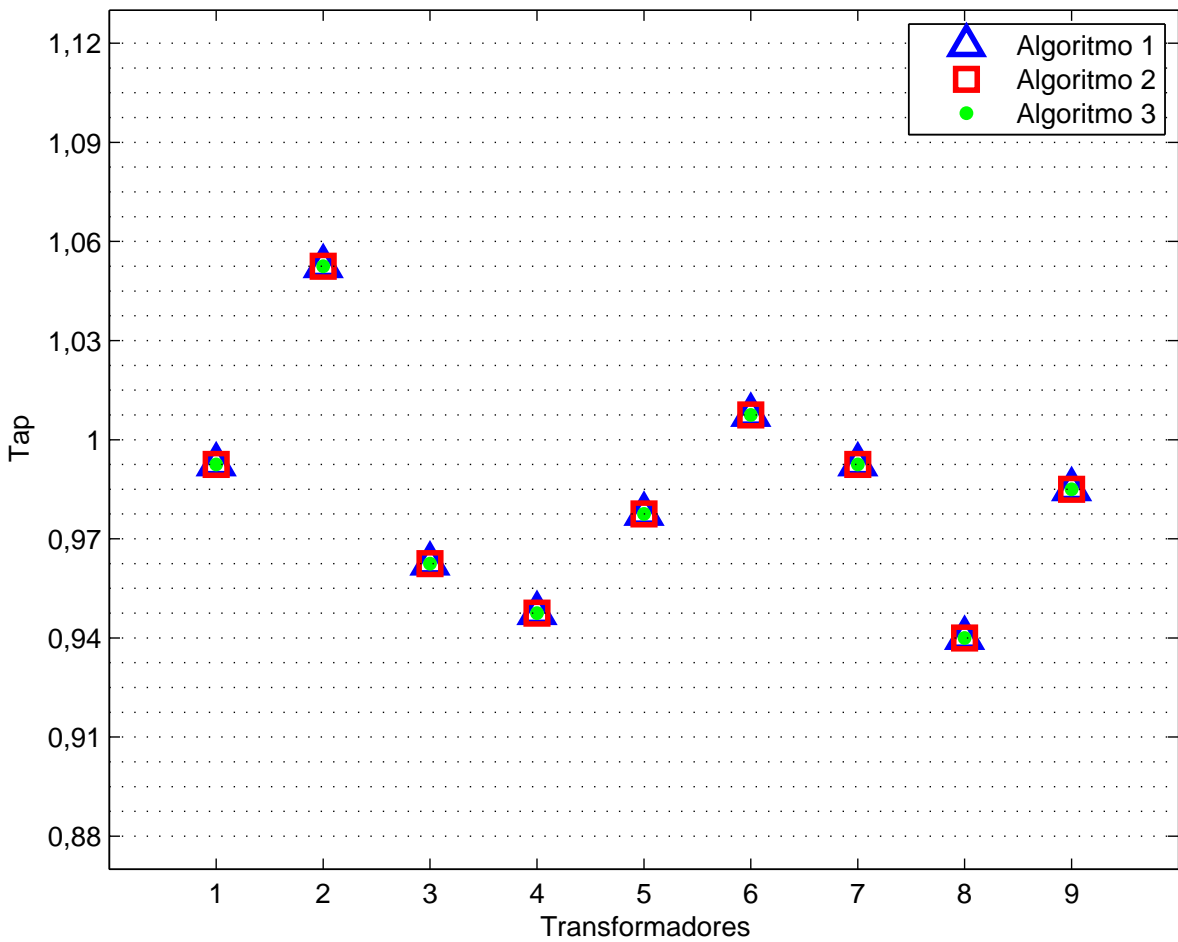

Figura 6.5 - Tap discreto para o sistema IEEE de 118 barras considerando a carga pesada. 


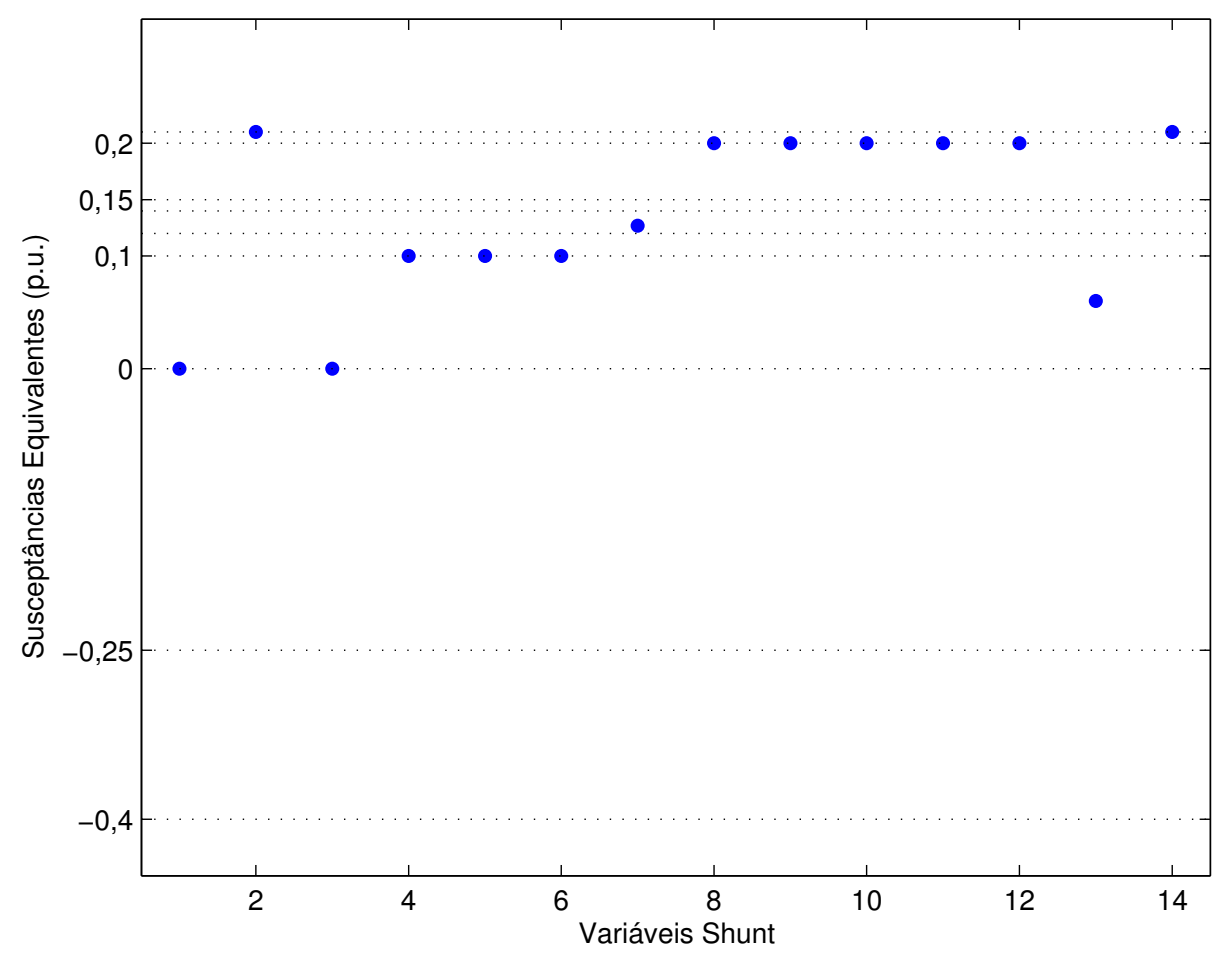

Figura 6.6 - Shunt contínuo para o sistema IEEE de 118 barras pelo modelo (2.6) considerando a carga pesada.

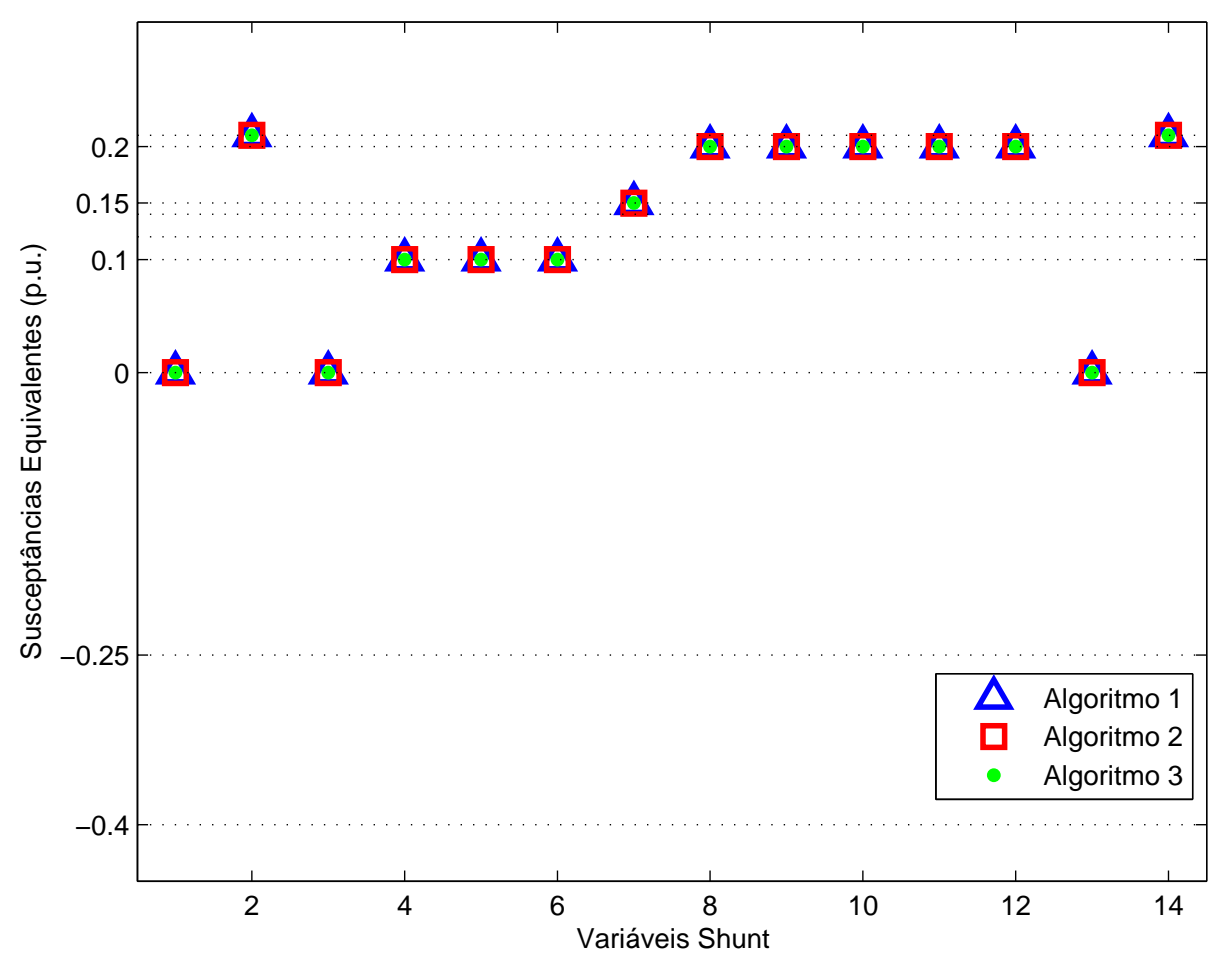

Figura 6.7 - Shunt discreto para o sistema IEEE de 118 barras considerando a carga pesada. 


\subsubsection{Sistema Elétrico IEEE de 300 Barras}

O sistema elétrico IEEE de 300 barras possui as seguintes características: 1 barra de geração (slack); 68 barras com controle de geração de reativos; 231 barras de carga; 8 bancos de capacitores; 6 reatores; 302 linhas de transmissão; e 107 transformadores com tap variável.

A Tabela 6.13, apresenta os valores discretos que cada variável shunt pode assumir quando considerada discreta, e os limitantes assumidos para a relaxação contínua.

Tabela 6.13 - Valores mínimo, máximo e discretos das susceptâncias e reatores shunts.

\begin{tabular}{ccccc}
\hline $\begin{array}{c}\text { Variáveis } \\
b_{k}^{s h}\end{array}$ & $\begin{array}{c}\text { Barra } \\
\text { Equivalente }\end{array}$ & $\begin{array}{c}\text { Limite Min } \\
\text { (p.u.) }\end{array}$ & $\begin{array}{c}\text { Limite Max } \\
\text { (p.u.) }\end{array}$ & $\begin{array}{c}\text { Conjunto de Valores } \\
\text { Discretos de } b_{k}^{s h}\end{array}$ \\
\hline 1 & 117 & 0.000 & 4.500 & $\{0.0,2,3.5,4.5\}$ \\
2 & 120 & 0.000 & 0.590 & $\{0.0,0.25,0.44,0.59\}$ \\
3 & 154 & 0.000 & 0.590 & $\{0.0,0.19,0.34,0.39\}$ \\
4 & 164 & -4.500 & 0.000 & $\{-4.5,0.0\}$ \\
5 & 166 & -4.500 & 0.000 & $\{-4.5,0.0\}$ \\
6 & 173 & 0.000 & 0.590 & $\{0.0,0.25,0.44,0.59\}$ \\
7 & 179 & 0.000 & 0.590 & $\{0.0,0.25,0.44,0.59\}$ \\
8 & 190 & -2.500 & 0.000 & $\{-2.5,0.0\}$ \\
9 & 231 & -4.500 & 0.000 & $\{-4.5,0.0\}$ \\
10 & 238 & -4.500 & 0.000 & $\{-4.5,0.0\}$ \\
11 & 240 & -1.500 & 0.000 & $\{-1.5,0.0\}$ \\
12 & 248 & 0.000 & 0.590 & $\{0.0,0.25,0.44,0.59\}$ \\
13 & 9003 & 0.000 & 0.150 & $\{0.0,0.15\}$ \\
14 & 9034 & 0.000 & 0.150 & $\{0.0,0.15\}$ \\
\hline
\end{tabular}

A Tabela 6.14 contêm os resultados do sistema IEEE de 300 barras considerando a Relaxação Contínua (2.6) e o modelo discreto (2.5) pelos os Algoritmos 1, 2 e 3. 
Tabela 6.14 - Resultados para o sistema IEEE de 300 barras.

\begin{tabular}{|c|c|c|c|}
\hline \multicolumn{4}{|c|}{ Relaxação Contínua } \\
\hline & Carga Leve & Carga Média & Carga Pesada \\
\hline Tempo (s) & 2.656 & 4.250 & 3.297 \\
\hline Perdas (MW) & 340.78 & 355.65 & 371.65 \\
\hline \multicolumn{4}{|c|}{ Parâmetros Iniciais } \\
\hline & Carga Leve & Carga Média & Carga Pesada \\
\hline$\gamma_{0}$ & 0.000001 & 0.0000001 & 0.000001 \\
\hline$c$ & 10 & 10 & 10 \\
\hline$\nu_{0}$ & 0.00058510 & 0.000060 & 0.00063016 \\
\hline$c_{1}$ & 4.5 & 3.5 & 4.5 \\
\hline \multicolumn{4}{|c|}{ Algoritmo 1 (Senoidal) } \\
\hline & Carga Leve & Carga Média & Carga Pesada \\
\hline Tempo (s) & 33.219 & 36.016 & 32.109 \\
\hline Número de Iterações & 10 & 11 & 10 \\
\hline Perdas (MW) & 344.95 & 359.13 & 374.60 \\
\hline \multicolumn{4}{|c|}{ Algoritmo 2 (Polinomial) } \\
\hline & Carga Leve & Carga Média & Carga Pesada \\
\hline Tempo (s) & 43.937 & 51.937 & 45.734 \\
\hline Número de Iterações & 10 & 13 & 10 \\
\hline Perdas (MW) & 345.91 & 359.87 & 377.25 \\
\hline \multicolumn{4}{|c|}{ Algoritmo 3 (Senoidal e Polinomial) } \\
\hline & Carga Leve & Carga Média & Carga Pesada \\
\hline Tempo (s) & 18.468 & 24.828 & 33.500 \\
\hline Número de Iterações & 6 & 8 & 9 \\
\hline Perdas (MW) & 341.81 & 356.61 & 373.00 \\
\hline
\end{tabular}

As Figuras 6.8 e 6.10 apresentam os resultados da relaxação contínua encontrados para as variáveis de controle tap dos transformadores e susceptância e reatores shunt considerando a carga pesada.

As Figuras 6.9 e 6.11 apresentam os resultados encontrados para as variáveis discretas de controle tapdos transformadores e susceptância e reatores shunt considerando a carga pesada para o modelo discreto (2.5). 


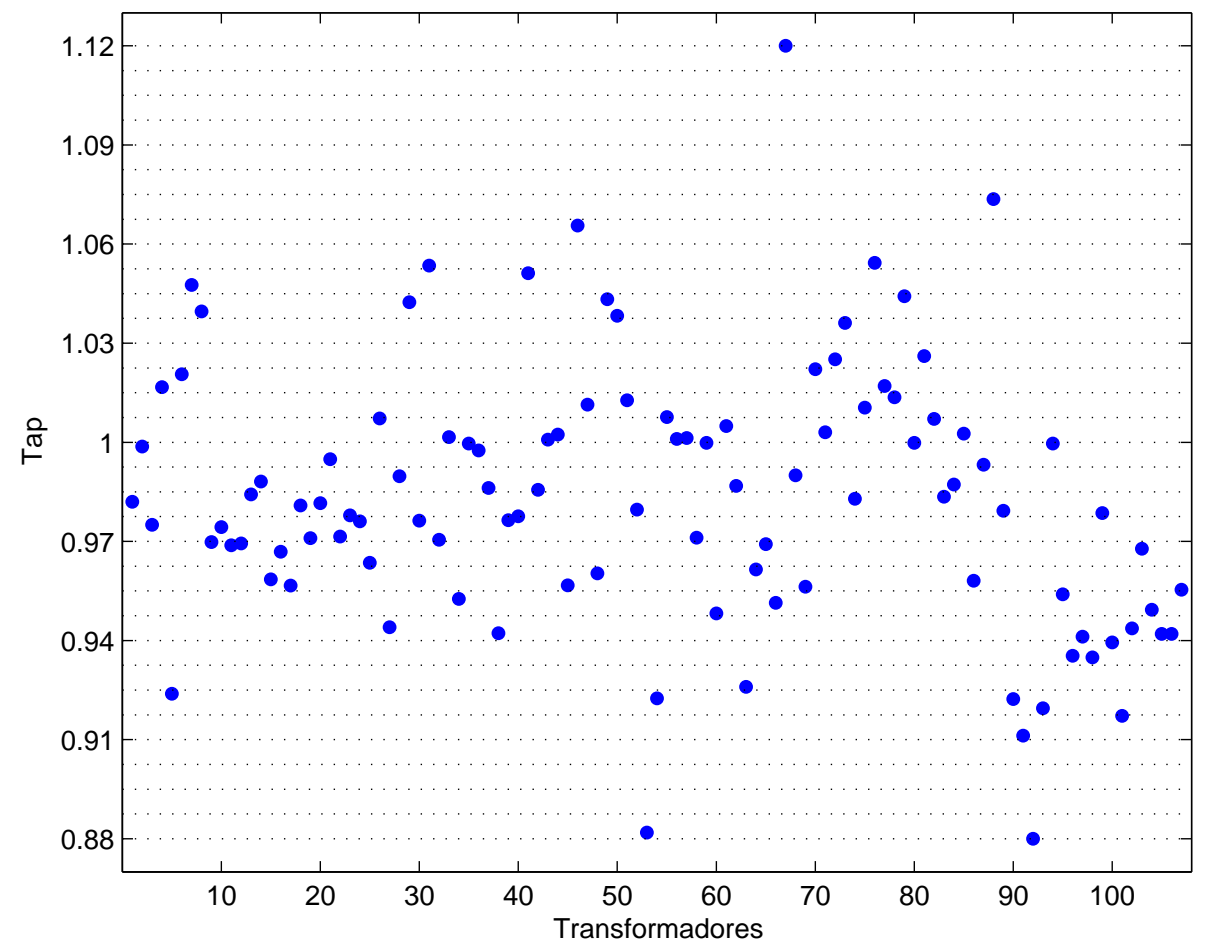

Figura 6.8 - Tap contínuo para o sistema IEEE de 300 barras pelo modelo (2.6) considerando a carga pesada.

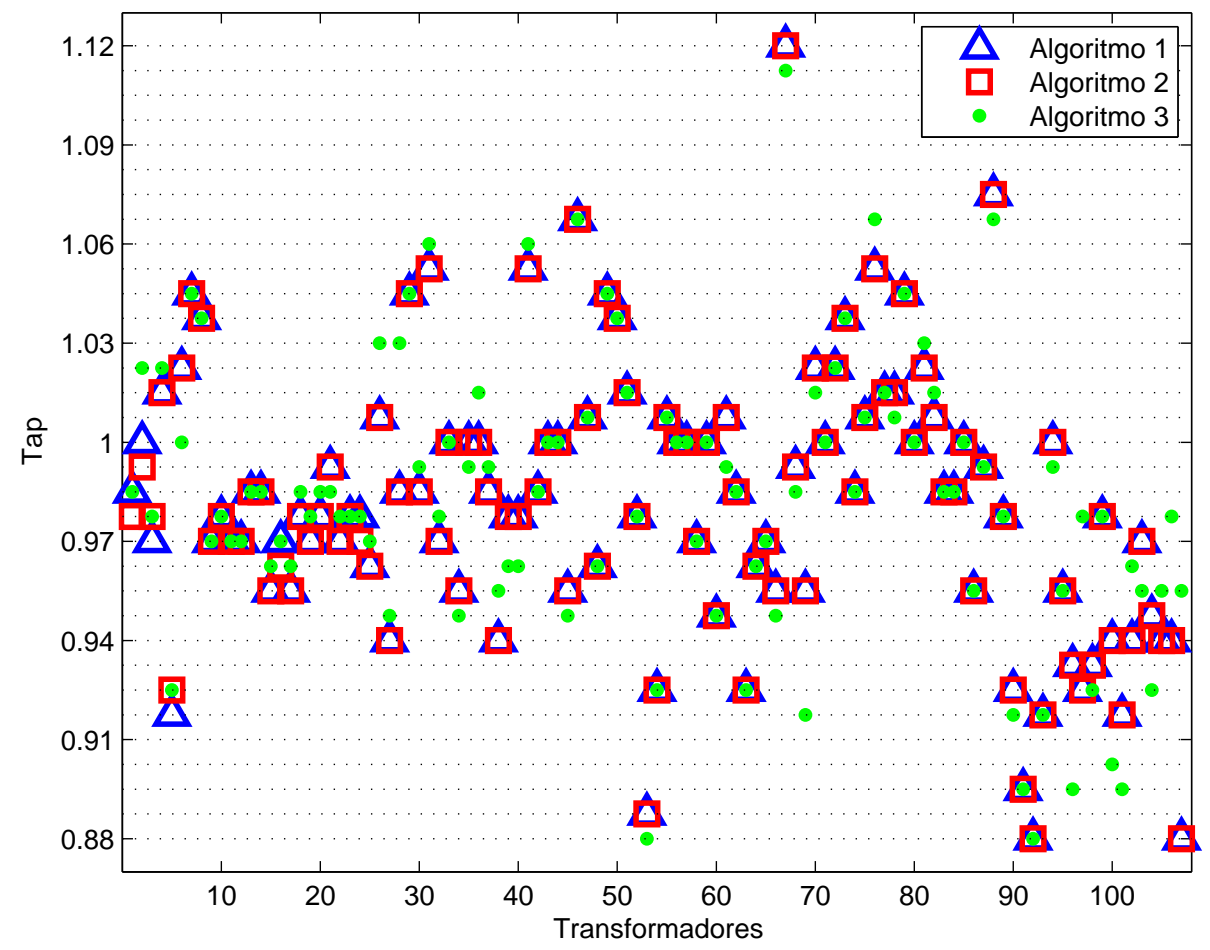

Figura 6.9 - Tap discreto para o sistema IEEE de 300 barras considerando a carga pesada. 


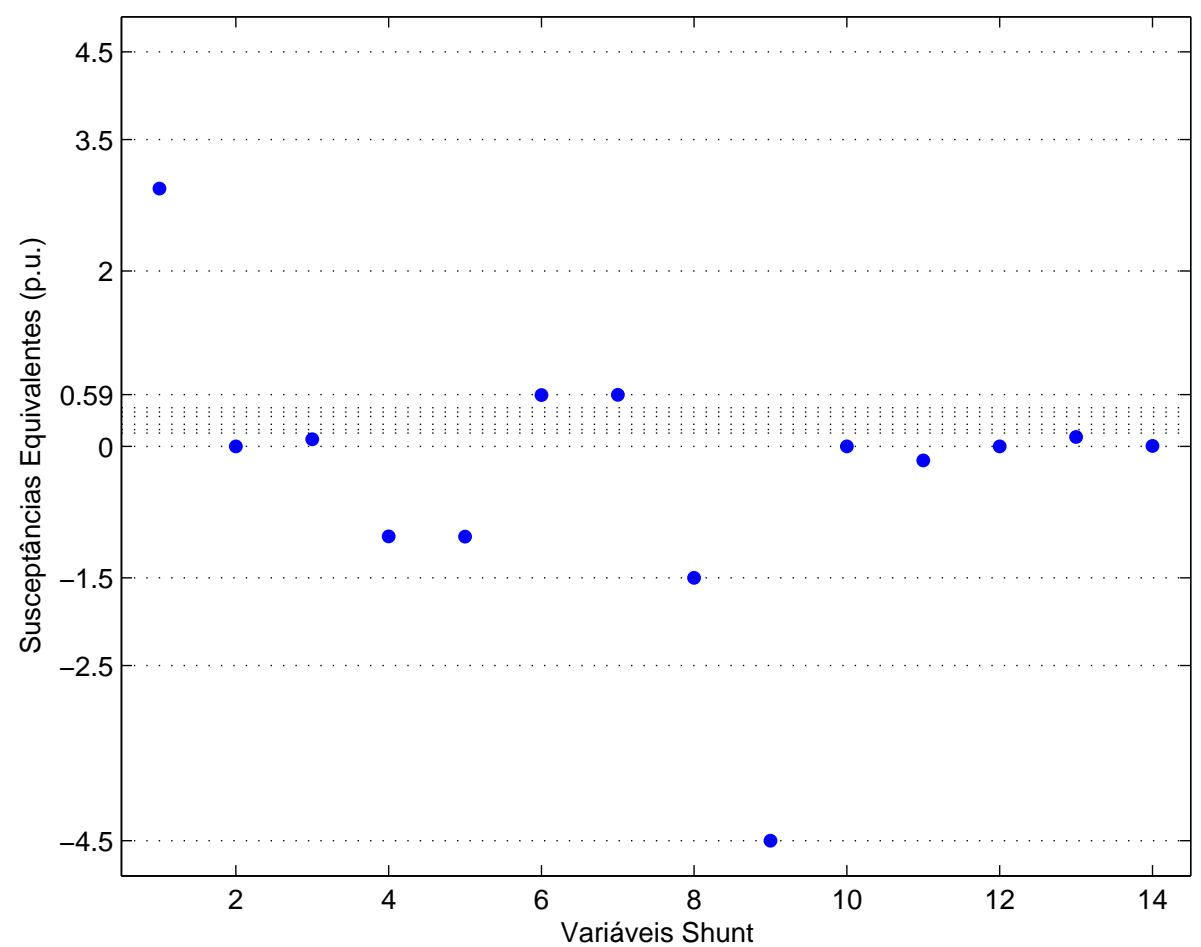

Figura 6.10 - Shunt contínuo para o sistema IEEE de 300 barras pelo modelo (2.6) considerando a carga pesada.

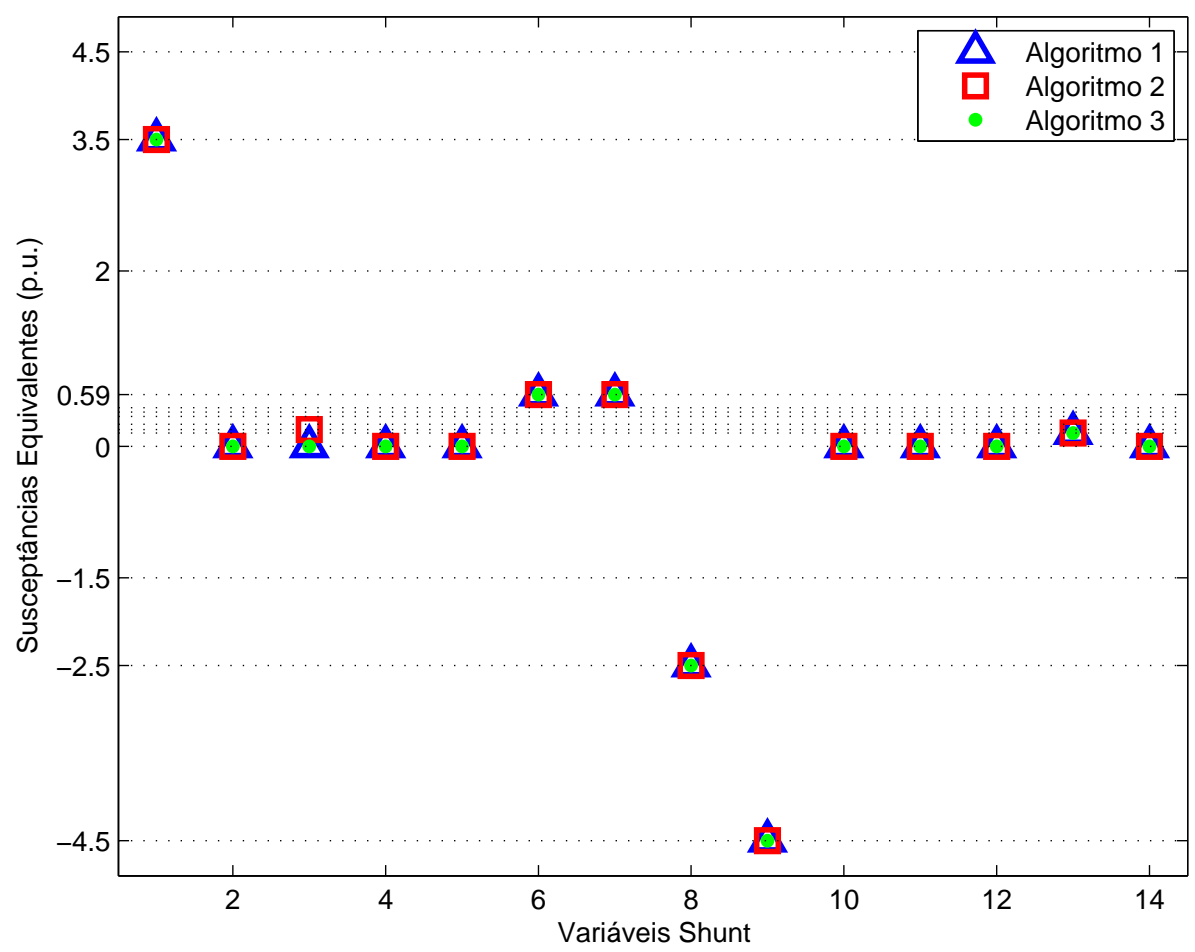

Figura 6.11 - Shunt discreto para o sistema IEEE de 300 barras considerando a carga pesada. 


\subsubsection{Análise dos Resultados}

Considerando cada sistema testado em todos os cenários de carregamento, é possível observar que, para os sistemas de 14, 30 e 118 barras, a aplicação de diferentes estratégias de discretização leva a soluções semelhantes. As perdas são quase as mesmas para todas as abordagens, com pequenas diferenças no tempo de execução e/ou o número de iterações. Além disso, em alguns casos, a mesma perda ativa é encontrada para diferentes ótimos locais. Uma possível razão é a baixa complexidade para encontrar uma solução discreta ideal para esses sistemas; pouco pode ser mencionado sobre esses resultados, além do fato de que as três abordagens de discretização são eficientes em encontrar uma solução ótima local de boa qualidade.

No entanto, para os sistemas de 57 e 300 barras, a estratégia mista, considerando ambas as funções de discretização senoidal e polinomial, é superior às outras em relação ao tempo computacional e em relação às perdas ativas, no caso das cargas leve e média. Por exemplo, para o sistema de 57 barras o tempo computacional da estratégia mista foi $16 \%$ menor que a senoidal e $29 \%$ menor que a polinomial para a carga leve, enquanto que para a carga média observou-se uma diferença de $8 \%$ e $42 \%$ respectivamente. Para o caso da carga pesada, os dois sistemas obtiveram um tempo levemente maior para a estratégia mista em relação ao encontrado pela senoidal e levemente menor em relação ao tempo encontrado pela polinomial, as perdas ativas foram melhores ou iguais para a estratégia mista.

A partir dos resultados obtidos, é possível observar, para todos os algoritmos, a eficácia do processo de solução independentemente da presença de variáveis discretas espaçadas regularmente ou da presença de variáveis discretas espaçadas por passos irregulares. Os algoritmos propostos são eficientes e adequados para a resolução de problemas de FPO com controles discretos e em geral a estratégia mista apresenta desempenho melhor ou igual as demais.

\subsubsection{Conclusões}

A estratégia proposta no Capítulo 3 explora o conceito de implantar funções de discretização eficientes para as variáveis discretas correspondentes, que poderiam, então, ser representadas por variáveis contínuas. Propõe-se a abordagem desenvolvida na Seção 3.3 usado a função senoidal para lidar com os taps de transformadores, que são ajustados por passos discretos regulares, e a função polinomial para lidar com os bancos de capacitores e reatores shunts, que são ajustados por passos discretos não regulares.

Os testes realizados com os sistemas-testes do IEEE mostram bom desempenho e eficiência para lidar com as variáveis discretas. As comparações entre as funções de discretização mostram que o desempenho da estratégia mista que pode fornecer boas soluções e tem potencial para superar as outras estratégias. Finalmente, a estratégia proposta é geral e apresenta potencial para resolver problemas com PNL com variáveis discretas em vários campos da 
engenharia.

A próxima seção contém os resultados numéricos obtidos utilizando o modelo de FPO com limitação do número de ações de controle. Os taps de transformadores considerados discretos são tratados pela função de discretização Polinomial e as variáveis binárias associadas as ações de controle são tratadas pela função Quadrática. 


\subsection{Limitação das Ações de Controles de um problema de FPO}

Nesta seção, apresentamos os resultados obtidos na resolução do modelo (2.8), no qual o problema de FPO com variáveis discretas, associadas aos taps de transformadores, possui uma limitação no número de ações de controle. Para o tratamento das variáveis binárias, associadas as ações de controle, utiliza-se a função quadrática apresentada na Seção 3.5. Já no tratamento das variáveis discretas utiliza-se a função de discretização polinomial descrita na Seção 3.2.

A Tabela 6.15 mostra as características de cada sistema para o modelo de FPO com variáveis contínuas e discretas considerando o número de ações de controle limitados (2.8).

Tabela 6.15 - Características dos Sistemas Elétricos

\begin{tabular}{c|cccc}
\hline $\begin{array}{c}\text { Sistema } \\
\text { Elétrico }\end{array}$ & $\begin{array}{c}\text { Restrições de } \\
\text { Igualdade }\end{array}$ & $\begin{array}{c}\text { Variáveis } \\
\text { Contínuas }\end{array}$ & $\begin{array}{c}\text { Variáveis } \\
\text { Discretas }\end{array}$ & $\begin{array}{c}\text { Variáveis } \\
\text { de Controle }\end{array}$ \\
\hline 14 & 22 & 27 & 3 & 8 \\
30 & 53 & 59 & 4 & 10 \\
57 & 106 & 113 & 15 & 22 \\
118 & 181 & 235 & 9 & 63 \\
300 & 530 & 599 & 107 & 176 \\
\hline
\end{tabular}

O número de iterações apresentado nas tabelas com os resultados representa um contador interno do algoritmo de discretização proposto utilizando a função polinomial, não estando relacionados com o número de iterações do solver escolhido.

O modelo modificado (5.1) é resolvido pelo solver CONOPT. Para estes testes, todos os valores inciais das variáveis inicialmente discretas (taps), já se iniciam discretos, e todos os sistemas testados são inicializados em pontos factíveis. $N^{\text {max }}$ é o número máximo de controles permitidos atuar e é determinado ao resolver o problema de FPO e calculando o número de controles necessários para minimizar as perdas. $N^{\text {min }}$ é calculado como o inteiro superior mais próximos de dois terços de $N^{\max }$.

Por motivo de comparação vamos realizar testes com o modelo (5.1) sem considerar a limitação no número de ações de controle (Número de Ações de Controle Ilimitados (NCI)).

A Tabela 6.16 contem os resultados obtidos ao se limitar o número de ações de controle e discretizar os taps como proposto inicialmente ${ }^{1}$.

\footnotetext{
${ }^{1}$ Estes resultados, foram apresentados no 2015 IEEE PES General Meeting realizado em Denver, Colorado, EUA, na seção Best Conference Papers (MAZZINI; ASADA; LAGE, 2015).
} 
Tabela 6.16 - Resultados numéricos para todos os sistemas IEEE

\begin{tabular}{c|ccccc}
\hline Sistemas: & 14 Barras & 30 Barras & 57 Barras & 118 Barras & 300 Barras \\
\hline$\nu^{(0)}$ & 0.000953 & 0.000022 & 0.000042 & 0.000037 & 0.000599 \\
$\ell$ & 4.5 & 4.5 & 3.5 & 4.5 & 4.5 \\
$N^{\text {min }}$ & 6 & 7 & 15 & 42 & 110 \\
$N^{\text {max }}$ & 8 & 10 & 22 & 63 & 165 \\
\hline \multicolumn{7}{c}{ Número de Ações de Controle Ilimitados (NCI) } \\
\hline Perdas $[\mathrm{MW}]$ & 12.28 & 16.02 & 22.38 & 106.19 & 342.94 \\
$\mathrm{~N}$ & 8 & 10 & 22 & 63 & 165 \\
Iterações & 2 & 2 & 2 & 2 & 2 \\
\hline \multicolumn{7}{c}{ Número de Ações de Controle Limitados (NCL) } \\
\hline Perdas $[\mathrm{MW}]$ & 12.31 & 17.10 & 24.55 & 124.96 & 350.45 \\
$\mathrm{~N}$ & 6 & 7 & 15 & 42 & 122 \\
Iterações & 3 & 3 & 8 & 7 & 6 \\
\hline
\end{tabular}

As Tabelas 6.17 e 6.18 apresentam a discretização obtida para os taps de transformadores dos sistemas IEEE de 14 e 30 Barras, respectivamente.

Tabela 6.17 - Resultados obtidos para os taps de transformadores do sistema IEEE de 14 Barras.

\begin{tabular}{c|ccc}
\hline Transformadores & Valores Iniciais & NCI & NCL \\
\hline 1 & 0.9775 & 1.0525 & 0.9775 \\
2 & 0.9700 & 0.8800 & 0.9700 \\
3 & 0.9325 & 0.9775 & 0.9625 \\
\hline
\end{tabular}

Tabela 6.18 - Resultados obtidos para os taps de transformadores do sistema IEEE de 30 Barras.

\begin{tabular}{c|ccc}
\hline Transformadores & Valores Iniciais & NCI & NCL \\
\hline 1 & 0.9775 & 1.0750 & 1.0600 \\
2 & 0.9700 & 0.9100 & 0.8800 \\
3 & 0.9325 & 1.0075 & 0.9325 \\
4 & 0.9700 & 0.9625 & 0.9625 \\
\hline
\end{tabular}


As Figuras 6.12, 6.13 e 6.14 contêm os taps de transformadores resultantes para os sistemas IEEE de 57, 118 e 300 Barras, respectivamente.

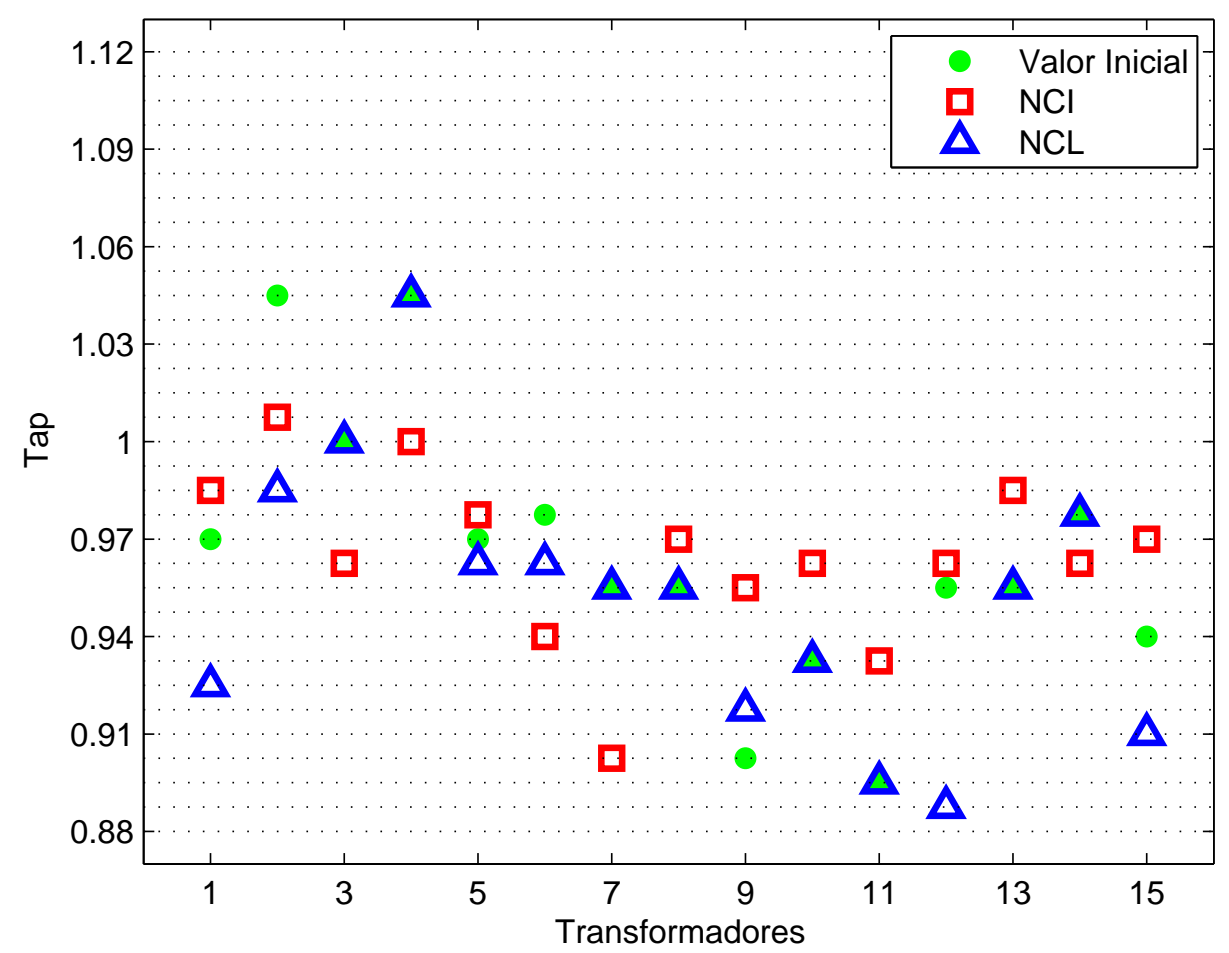

Figura 6.12 - Valor Inicial, NCI e NCL para os taps do sistema de 57 Barras.

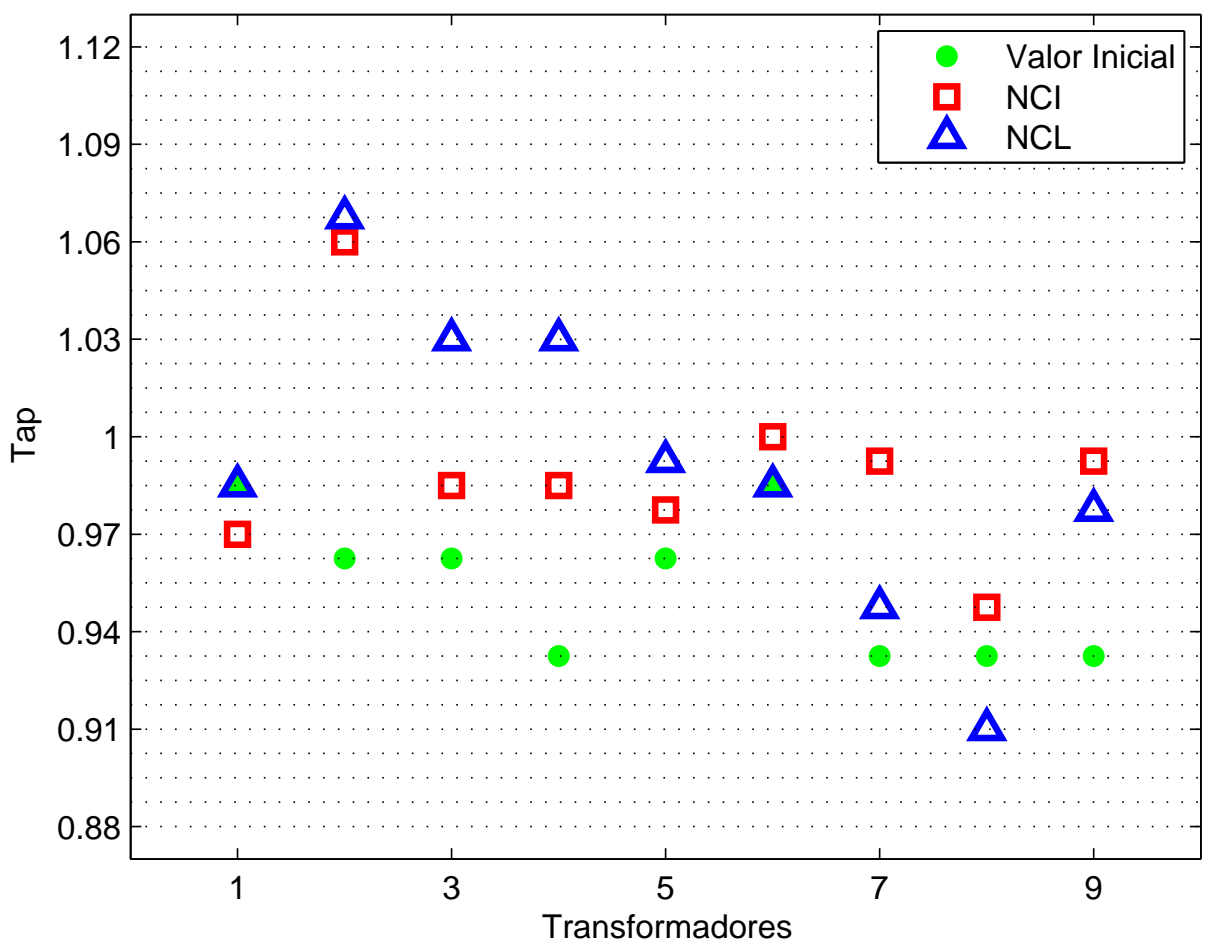

Figura 6.13 - Valor Inicial, NCI e NCL para os taps do sistema de 118 Barras. 


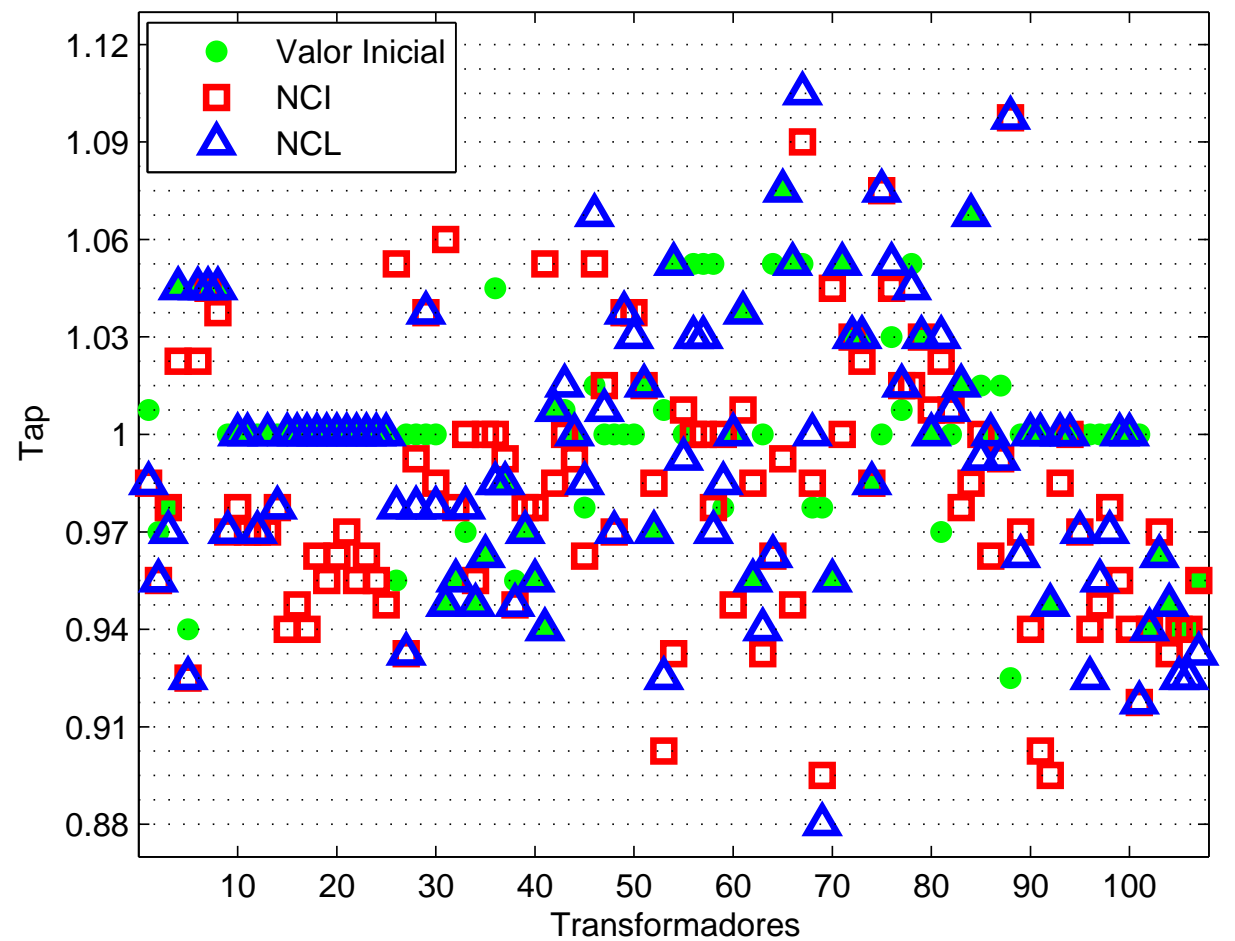

Figura 6.14 - Valor Inicial, NCI e NCL para os taps do sistema de 300 Barras.

\subsubsection{Análise dos Resultados}

As perdas de potência ativa encontradas com Número de Ações de Controle Ilimitados (NCI) são consideradas um limite inferior para as perdas de potência ativa do caso em que ocorre um Número de Ações de Controle Limitados (NCL). O número de ações de controle encontrado para os testes com NCI corresponde ao limite superior $N^{\max }$ para as ações de controle do caso NCL.

Considerando o sistema IEEE de 14 barras, todos os taps foram modificados no teste com NCI, enquanto que para o caso com NCL apenas um tap atuou. Contudo, as perdas de potência ativa cresceram apenas 0.03 MW de NCI para NCL, e o número de ações de controle diminuiu em 2. Para o sistema IEEE de 57 barras tais diferenças são mais acentuadas e para o sistema IEEE de 300 barras, de 107 possíveis controles para os taps de transformadores, 96 efetivamente mudaram para NCI e 53 mudaram para o caso NCL considerando o valor inicial; o número de ações de controle diminuiu em 43 de NCI para NCL, enquanto as perdas aumentaram em 7.51 MW.

Para todos os sistemas, a magnitude da tensão dos geradores e compensadores síncronos sempre mudou independentemente do estado inicial do sistema.

\subsubsection{Conclusões}

A abordagem para resolver os problemas de FPO apresentados nesta seção permite que as variáveis de controles discretas sejam consideradas como contínuas em um problema 
modificado, proporcionando um menor número de ajustes aplicáveis durante um período de tempo.

Os testes numéricos realizados com os sistemas teste do IEEE têm mostrado bons resultados e eficácia em lidar com variáveis discretas e binárias. A fim de analisar a eficácia das abordagens, em primeiro lugar a função polinomial de discretização foi analisada sem considerar um limitado número de ações de controle. Depois os mesmos testes foram realizados considerando a limitação do número de ações de controles. O trade-off entre a realização ideal da função de objetivo, satisfazendo as restrições operacionais e reduzindo o número de ações de controles resultaram em boas soluções.

A próxima seção é subdividida em três subseções contendo os resultados numéricos para o problema multiobjetivo.

- Subseção 6.3.2 - Resultados consideramos o problema de FPO multiobjetivo com taps de transformadores e banco de capacitores e reatores shunts contínuos. Os resultados são divididos em duas subseções:

- Resultados para o modelo modificado pelo método da Soma Ponderada, onde as variáveis binárias são modeladas pela função Sigmoidal.

- Resultados para o modelo modificado pelo método do $\epsilon$-Restrito, onde as variáveis binárias são modeladas pela função Quadrática.

- Subseção 6.3.2 - Resultados para o problema de FPO multiobjetivo com taps de transformadores discretos e banco de capacitores e reatores shunts contínuos modificado pelo método $\epsilon$-Restrito. As variáveis discretas são modeladas pela função quadrática e os taps de transformadores são modelados pela função de discretização polinomial.

- Subseção 6.3.3 - Resultados para o problema de FPO multiobjetivo com taps de transformadores discretos e banco de capacitores e reatores shunts discretos modificado pelo método da soma ponderada. As variáveis discretas são tratadas pelas funções de discretização e as variáveis binárias pela função Sigmoidal. 


\subsection{FPO Multiobjetivo}

Os resultados apresentados nesta seção estão dispostos em três partes: FPO Multiobjetivo com taps de transformadores contínuos e os bancos de capacitores e reatores shunts fixos (2.14); FPO Multiobjetivo com taps de transformadores discretos e os bancos de capacitores e reatores shunts fixos (5.7); e FPO Multiobjetivo com taps de transformadores e os bancos de capacitores e reatores shunts discretos (2.15).

Usaremos dois métodos para o tratamento do modelo de FPO multiobjetivo, em ambos os métodos ocorre a modificação do problema original em um problema mono-objetivo, permitindo o uso de técnicas clássicas de otimização não linear na resolução do problema. Os métodos utilizados são: Método da Soma Ponderada e Método $\epsilon$-Restrito.

No caso da Soma Ponderada, devemos inicialmente estabelecer pesos para os objetivos, que normalizados são agrupados em um único objetivo. Como já mencionado, o problema de FPO multiobjetivo em questão possui apenas duas funções objetivo, desta forma o método da soma ponderada é modelado com apenas dois pesos, o que pode ser feito com apenas um fator de ponderação $\omega(\omega+(1-\omega)=1)$. A desvantagem em utilizar este método consiste na impossibilidade de testar todos os pesos reais de 0 a 1 . Devido a isto, consideramos 11 casos de testes com diferentes pesos $\omega$ variando de 0 a 1 por passos de 0.1 . Para o sistema IEEE de 118 barras foi necessário testes com mais valores de $\omega$, estes valores são especificados nos resultados. Quando consideramos $\omega=0$ ou $\omega=1$, não obtemos uma solução para o problema multiobjetivo, já que para $\omega=0$ apenas o número de ações de controles é minimizado, e quando $\omega=0$ apenas as perdas ativas na transmissão é minimizada. Esses resultados considerando $\omega=0$ e $\omega=1$ são utilizados apenas como limitantes para os demais testes.

Ao utilizar o método $\epsilon$-Restrito, consideramos como função objetivo apenas as Perdas Ativas na transmissão, o número de ações de controle torna-se uma restrição do problema modificado, e os valores para $\epsilon$ são atribuídos de acordo com o número de ações de controle de cada sistema. Para os sistemas IEEE de 14, 30 e 57 barras foram testados todos os valores para $\epsilon$, já os sistemas IEEE de 118 e 300 barras foram selecionados alguns valores de $\epsilon$. A maior desvantagem em utilizar este método consiste justamente no fato de que um determinado $\epsilon$ pode tornar o problema infactível, ou seja, não há garantias de que vamos encontrar soluções factíveis para todos os valores de $\epsilon$.

Para a modelagem das variáveis binárias, utilizamos a função Sigmoidal apresentada na Seção 3.4 e a função Quadrática apresentada na Seção 3.5. A função Sigmoidal é utilizada junto com o método da Soma Ponderada enquanto a função Quadrática é utilizada junto do Método $\epsilon-$ Restrito.

Devemos deixar claro que para o caso contínuo os taps de transformadores são inicializados conforme o banco de dados base IEEE. Já para o caso discreto, como consideramos minimizar o número de ações de controle, os valores iniciais das variáveis discretas do problema original (taps e shunts), já se iniciam em valores discretos, e todos os sistemas testados são inicializados em pontos factíveis. 
A próxima seção contém os resultados considerando o problema de FPO multiobjetivo com taps de transformadores e banco de capacitores e reatores shunts contínuos. Este resultados são divididos em duas subseções:

- Resultados para o modelo modificado pelo método da Soma Ponderada, onde as variáveis binárias são modeladas pela função Sigmoidal.

- Resultados para o modelo modificado pelo método do $\epsilon$-Restrito, onde as variáveis binárias são modeladas pela função Quadrática. 


\subsubsection{FPO Multiobjetivo com Tap de Transformadores Contínuos}

Nesta seção apresentamos os resultados encontrados para o modelo de FPO Multiobjetivo com taps de transformadores contínuos e os bancos de capacitores e reatores shunts fixos (2.14). As variáveis de controle são formadas pelas tensões e pelos taps de transformadores, e para cada variável de controle está associada uma variável binária.

A Tabela 6.19 mostra as características de cada sistema para o modelo (2.14).

Tabela 6.19 - Características dos Sistemas Elétricos

\begin{tabular}{c|cccc}
\hline $\begin{array}{c}\text { Sistema } \\
\text { Elétrico }\end{array}$ & $\begin{array}{c}\text { Restrições de } \\
\text { Igualdade }\end{array}$ & $\begin{array}{c}\text { Variáveis } \\
\text { Contínuas }\end{array}$ & $\begin{array}{c}\text { Variáveis de } \\
\text { Controle }\end{array}$ & $\begin{array}{c}\text { Variáveis } \\
\text { Binárias }\end{array}$ \\
\hline 14 & 22 & 30 & 8 & 8 \\
30 & 53 & 63 & 10 & 10 \\
57 & 106 & 219 & 22 & 22 \\
118 & 181 & 416 & 63 & 63 \\
300 & 530 & 1129 & 176 & 176 \\
\hline
\end{tabular}

O número de iterações apresentado nas tabelas desta seção representa um contador interno do algoritmo proposto utilizando a função sigmoidal, não estando relacionados com o número de iterações do solver escolhido.

\subsubsection{Método da Soma Ponderada}

O modelo de FPO Multiobjetivo com taps de transformadores contínuos e os bancos de capacitores e reatores shunts fixos (2.14) é modificado em um problema mono-objetivo pelo método da soma ponderada como em (5.2), podendo ser resolvido por métodos clássicos de PNLIM, assim como pelos métodos implementados nos solvers DICOPT e KNITRO. Estes solvers possuem estratégias de resoluções que tratam as variáveis binárias do problema (5.2). Os testes realizados com DICOPT e KNITRO foram elaborados como critério de comparação com a estratégia de resolução desenvolvida, utilizando a função Sigmoidal (3.4) para o tratamento das variáveis binárias. As variáveis binárias do problema (5.2) são modificadas pela função Sigmoidal como em (5.3). O problema (5.3) é não linear com variáveis contínuas, e será resolvido pelo solver CONOPT. No Algoritmo 5, uma sequência de problemas modificados como (5.3) é resolvido por CONOPT até que todas as variáveis associadas às variáveis binárias do problema original assumam valores binários.

As Tabelas 6.33, 6.34, 6.35, 6.36 e 6.37 apresentam os resultados obtidos para os sistemas IEEE de 14, 30, 57, 118 e 300 barras, respectivamente. $\Delta \mathrm{N}$ é a diferença entre o número de ações de controle para o peso atual e número de ações de controle do peso anterior e $\Delta \mathrm{P}$ é a diferença entre as perdas ativas para o peso atual e as perdas ativas para o peso anterior. Matematicamente, $\Delta \mathrm{N}$ e $\Delta \mathrm{P}$ podem ser calculados como:

$$
\Delta \mathrm{N}=\mathrm{N}\left(\omega_{i}\right)-\mathrm{N}\left(\omega_{i-1}\right)
$$


e

$$
\Delta \mathrm{P}=\operatorname{Perda}\left(\omega_{i}\right)-\operatorname{Perda}\left(\omega_{i-1}\right)
$$

onde $\mathrm{N}\left(\omega_{i}\right)$ é o número de ações de controle encontrado para o peso $\omega_{i}$ e Perda $\left(\omega_{i}\right)$ é a perda ativa encontrada para o peso $\omega_{i}$.

Tabela 6.20 - Resultados do sistema IEEE de 14 barras para os diferentes valores de $\omega$.

\begin{tabular}{|c|c|c|c|c|c|c|c|c|c|c|c|}
\hline$\omega$ & 0.0 & 0.1 & 0.2 & 0.3 & 0.4 & 0.5 & 0.6 & 0.7 & 0.8 & 0.9 & 1.0 \\
\hline \multicolumn{12}{|c|}{ DICOPT } \\
\hline$\overline{\mathrm{N}}$ & 0 & $\overline{0}$ & 0 & 0 & 4 & 4 & 4 & 4 & 4 & 6 & 8 \\
\hline Perdas $[\mathrm{MW}]$ & 13.39 & 13.39 & 13.39 & 13.39 & 12.34 & 12.34 & 12.34 & 12.34 & 12.34 & 12.29 & 12.28 \\
\hline$\Delta \mathrm{N}$ & - & 0 & 0 & 0 & 4 & 0 & 0 & 0 & 0 & 2 & 2 \\
\hline$\Delta \mathrm{P}[\mathrm{MW}]$ & - & 0 & 0 & 0 & -1.05 & 0 & 0 & 0 & 0 & -0.05 & -0.01 \\
\hline Tempo (s) & 0.194 & 0.243 & 0.665 & 1.094 & 0.410 & 0.627 & 0.610 & 0.399 & 0.823 & 0.645 & 0.256 \\
\hline \multicolumn{12}{|c|}{ KNITRO } \\
\hline$\overline{\mathrm{N}}$ & 0 & 0 & 0 & 2 & 4 & 4 & 5 & 5 & 5 & 5 & 8 \\
\hline Perdas [MW] & 13.39 & 13.39 & 13.39 & 12.88 & 12.34 & 12.34 & 12.31 & 12.31 & 12.31 & 12.31 & 12.28 \\
\hline$\Delta \mathrm{N}$ & - & 0 & 0 & 2 & 2 & 0 & 0 & 0 & 0 & 0 & 3 \\
\hline$\Delta \mathrm{P}[\mathrm{MW}]$ & - & 0 & 0 & -0.51 & -0.54 & 0 & $-0,03$ & 0 & 0 & 0 & -0.03 \\
\hline Tempo (s) & 0.306 & 0.298 & 0.305 & 0.422 & 0.302 & 0.314 & 0.308 & 0.313 & 0.307 & 0.304 & 0.309 \\
\hline \multicolumn{12}{|c|}{ Algoritmo 5 e CONOPT } \\
\hline$\overline{\mathrm{N}}$ & 0 & 0 & 0 & 2 & 4 & 4 & 4 & 4 & 4 & 5 & 8 \\
\hline Perdas [MW] & 13.39 & 13.39 & 13.39 & 12.88 & 12.34 & 12.34 & 12.34 & 12.34 & 12.34 & 12.31 & 12.28 \\
\hline$\Delta \mathrm{N}$ & - & 0 & 0 & 2 & 2 & 0 & 0 & 0 & 0 & 1 & 3 \\
\hline$\Delta \mathrm{P}[\mathrm{MW}]$ & - & 0 & 0 & -0.51 & -0.54 & 0 & 0 & 0 & 0 & -0.03 & -0.03 \\
\hline Iterações & 2 & 2 & 2 & 2 & 2 & 2 & 2 & 3 & 3 & 3 & 3 \\
\hline Tempo $[\mathrm{s}]$ & 0.245 & 0.207 & 0.238 & 0.279 & 0.251 & 0.244 & 0.225 & 0.219 & 0.479 & 0.245 & 0.363 \\
\hline
\end{tabular}

Tabela 6.21 - Resultados do sistema IEEE de 30 barras para os diferentes valores de $\omega$.

\begin{tabular}{|c|c|c|c|c|c|c|c|c|c|c|c|}
\hline $\bar{\omega}$ & 0.0 & 0.1 & 0.2 & 0.3 & 0.4 & 0.5 & 0.6 & 0.7 & 0.8 & 0.9 & 1.0 \\
\hline \multicolumn{12}{|c|}{ DICOPT } \\
\hline$\overline{\mathrm{N}}$ & 0 & 0 & 0 & 1 & 4 & 4 & 4 & 4 & 6 & 8 & 10 \\
\hline Perdas [MW] & 17.54 & 17.54 & 17.54 & 17.23 & 16.31 & 16.31 & 16.31 & 16.31 & 16.07 & 16.03 & 16.02 \\
\hline$\Delta \mathrm{N}$ & - & 0 & 0 & 1 & 3 & 0 & 0 & 0 & 2 & 2 & 2 \\
\hline$\Delta \mathrm{P}[\mathrm{MW}]$ & - & 0 & 0 & -0.31 & -0.92 & 0 & 0 & 0 & -0.24 & -0.04 & -0.01 \\
\hline Tempo $[\mathrm{s}]$ & 0.097 & 0.231 & 0.348 & 0.311 & 0.956 & 0.744 & 0.802 & 0.992 & 0.936 & 0.783 & 0.173 \\
\hline \multicolumn{12}{|c|}{ KNITRO } \\
\hline$\overline{\mathrm{N}}$ & 0 & 0 & 0 & 2 & 2 & 4 & 6 & 6 & 7 & 8 & 9 \\
\hline Perdas [MW] & 17.54 & 17.54 & 17.54 & 16.90 & 16.90 & 16.31 & 16.07 & 16.07 & 16.06 & 16.03 & 16.02 \\
\hline$\Delta \mathrm{N}$ & - & 0 & 0 & 2 & 0 & 2 & 2 & 0 & 1 & 1 & 1 \\
\hline$\Delta \mathrm{P}[\mathrm{MW}]$ & - & 0 & 0 & -0.64 & 0 & -0.59 & -0.24 & 0 & -0.01 & -0.03 & -0.01 \\
\hline Tempo $[\mathrm{s}]$ & 0.105 & 0.187 & 0.184 & 0.338 & 0.200 & 0.195 & 0.306 & 0.217 & 0.242 & 0.257 & 0.134 \\
\hline \multicolumn{12}{|c|}{ Algoritmo 5 e CONOPT } \\
\hline$\overline{\mathrm{N}}$ & 0 & 0 & 0 & 2 & 4 & 4 & 5 & 6 & 6 & 8 & 10 \\
\hline Perdas $[\mathrm{MW}]$ & 17.54 & 17.54 & 17.54 & 16.90 & 16.31 & 16.31 & 16.19 & 16.07 & 16.07 & 16.03 & 16.02 \\
\hline$\Delta \mathrm{N}$ & - & 0 & 0 & 2 & 2 & 0 & 1 & 1 & 0 & 2 & 2 \\
\hline$\Delta \mathrm{P}[\mathrm{MW}]$ & - & 0 & 0 & -0.64 & -0.59 & 0 & -0.12 & -0.12 & 0 & -0.04 & -0.01 \\
\hline Iterações & 2 & 2 & 2 & 2 & 2 & 2 & 2 & 2 & 2 & 2 & 2 \\
\hline Tempo $[\mathrm{s}]$ & 0.251 & 0.256 & 0.204 & 0.213 & 0.238 & 0.231 & 0.245 & 0.230 & 0.239 & 0.232 & 0.311 \\
\hline
\end{tabular}


Tabela 6.22 - Resultados do sistema IEEE de 57 barras para os diferentes valores de $\omega$. Soluções com * são dominadas pelas demais.

\begin{tabular}{|c|c|c|c|c|c|c|c|c|c|c|c|}
\hline$\omega$ & 0.0 & 0.1 & 0.2 & 0.3 & 0.4 & 0.5 & 0.6 & 0.7 & 0.8 & 0.9 & 1.0 \\
\hline \multicolumn{12}{|c|}{ DICOPT } \\
\hline $\mathrm{N}$ & 1 & 6 & 7 & 8 & 8 & 9 & 9 & 13 & 14 & $16^{*}$ & 22 \\
\hline Perdas $[\mathrm{MW}]$ & 26.31 & 24.90 & 22.94 & 22.03 & 22.03 & 21.91 & 21.91 & 21.61 & 21.30 & 21.31 & 21.19 \\
\hline$\Delta N$ & - & 5 & 1 & 1 & 0 & 1 & 0 & 4 & 1 & 2 & 6 \\
\hline$\Delta \mathrm{P}[\mathrm{MW}]$ & - & -1.41 & -1.96 & -0.91 & 0 & -0.12 & 0 & -0.30 & -0.31 & 0.01 & -0.12 \\
\hline Tempo [s] & 0.171 & 0.765 & 1.561 & 1.323 & 1.466 & 0.987 & 1.111 & 2.670 & 1.653 & 1.872 & 0.773 \\
\hline \multicolumn{12}{|c|}{ KNITRO } \\
\hline $\mathrm{N}$ & $1^{*}$ & 2 & 1 & 4 & 10 & 8 & $13^{*}$ & 13 & 16 & 17 & 21 \\
\hline Perdas $[\mathrm{MW}]$ & 26.08 & 25.63 & 26.00 & 25.38 & 21.86 & 22.03 & 21.68 & 21.40 & 21.29 & 21.26 & 21.33 \\
\hline$\Delta \mathrm{N}$ & - & 1 & -1 & 3 & 6 & -2 & 5 & 0 & 3 & 1 & 4 \\
\hline$\Delta \mathrm{P}[\mathrm{MW}]$ & - & -0.45 & 0.37 & -0.62 & -3.52 & 0.17 & -0.35 & -0.28 & -0.11 & -0.03 & 0.07 \\
\hline Tempo $[\mathrm{s}]$ & 0.320 & 0.754 & 0.319 & 0.585 & 0.634 & 1.037 & 1.342 & 2.310 & 4.014 & 4.585 & 0.218 \\
\hline \multicolumn{12}{|c|}{ Algoritmo 5 e CONOPT } \\
\hline$\overline{\mathrm{N}}$ & $1^{*}$ & 1 & $\overline{1}$ & 8 & 8 & 8 & 9 & 13 & 14 & 17 & 22 \\
\hline Perdas $[\mathrm{MW}]$ & 26.32 & 26.21 & 26.21 & 22.03 & 22.03 & 22.03 & 21.97 & 21.41 & 21.30 & 21.26 & 21.19 \\
\hline$\Delta \mathrm{N}$ & - & 0 & 0 & 7 & 0 & 0 & 1 & 4 & 1 & 3 & 5 \\
\hline$\Delta \mathrm{P}[\mathrm{MW}]$ & - & -0.11 & 0 & -4.18 & 0 & 0 & -0.06 & -0.56 & -0.11 & -0.04 & -0.07 \\
\hline Iterações & 4 & 4 & 4 & 3 & 3 & 3 & 3 & 3 & 3 & 3 & 3 \\
\hline Tempo $[\mathrm{s}]$ & 0.475 & 0.480 & 0.517 & 0.434 & 0.434 & 0.634 & 0.740 & 0.643 & 0.699 & 0.864 & 0.722 \\
\hline
\end{tabular}


Tabela 6.23 - Resultados do sistema IEEE de 118 barras para os diferentes valores de $\omega$. Soluções com * são dominadas pelas demais.

\begin{tabular}{|c|c|c|c|c|c|c|c|c|c|c|c|c|c|c|c|c|c|}
\hline$\omega$ & 0.0 & 0.1 & 0.2 & 0.3 & 0.4 & 0.42 & 0.44 & 0.46 & 0.48 & 0.49 & 0.495 & 0.5 & 0.6 & 0.7 & 0.8 & 0.9 & 1.0 \\
\hline & & & & & & & & DICOP & & & & & & & & & \\
\hline 14 & & $15^{*}$ & 13 & 11 & & 9 & 10 & $13^{*}$ & 28 & $\overline{42}$ & 34 & 32 & 47 & 55 & $58^{*}$ & 58 & 63 \\
\hline $\begin{array}{l}\text { Perdas }[\mathrm{MW}] \\
\Delta \mathrm{N}\end{array}$ & 132.42 & $\begin{array}{c}129.81 \\
12\end{array}$ & $\begin{array}{c}129.77 \\
-2\end{array}$ & $\begin{array}{l}131.15 \\
-2\end{array}$ & $\begin{array}{l}131.65 \\
-4\end{array}$ & $\begin{array}{c}131.57 \\
2\end{array}$ & $\begin{array}{c}131.53 \\
1\end{array}$ & $\begin{array}{c}130.75 \\
3\end{array}$ & $\begin{array}{c}128.03 \\
15\end{array}$ & $\begin{array}{c}119.53 \\
14\end{array}$ & $\begin{array}{c}121.86 \\
8\end{array}$ & $\begin{array}{l}122.25 \\
-2\end{array}$ & $\begin{array}{c}117.71 \\
15\end{array}$ & $\begin{array}{c}112.06 \\
8\end{array}$ & $\begin{array}{c}106.93 \\
3\end{array}$ & $\begin{array}{l}106.72 \\
0\end{array}$ & $\begin{array}{c}06.15 \\
5\end{array}$ \\
\hline$\Delta \mathrm{P}[\mathrm{MW}]$ & - & -2.62 & -0.03 & 1.38 & 0.50 & -0.08 & -0.04 & -0.78 & -2.72 & -8.50 & 2.33 & -9.40 & -4.54 & -5.65 & -5.13 & -0.21 & -0.57 \\
\hline Tempo [s] & 1.549 & 4.151 & 5.438 & 5.695 & 312.735 & 701.101 & 1000.795 & 814.987 & 45.161 & 87.005 & 101.035 & 91.084 & 304.248 & 72.392 & 7.340 & 26.606 & 1.001 \\
\hline \multicolumn{18}{|c|}{ KNITRO } \\
\hline & $3^{*}$ & $3^{*}$ & 3 & 2 & 8 & $9^{*}$ & 10 & 14 & $21^{*}$ & - & 25 & & 56 & & & 60 & 62 \\
\hline Perdas [MW] & 132.47 & 132.15 & 131.90 & 132.18 & 128.08 & 128.68 & 127.25 & 126.67 & 126.91 & - & 124.10 & - & 107.06 & 106.38 & 106.25 & 106.24 & 106.15 \\
\hline$\Delta \mathrm{N}$ & - & 0 & 0 & -1 & 6 & 1 & 1 & 3 & 7 & ( & 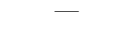 & 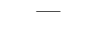 & 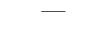 & 3 & & 0 & 2 \\
\hline $\begin{array}{l}\Delta \mathrm{P}[\mathrm{MW}] \\
\text { Tempo [s] }\end{array}$ & $5 . \overline{158}$ & $\begin{array}{l}-0.32 \\
1.954\end{array}$ & $\begin{array}{l}-0.25 \\
18.794\end{array}$ & $\begin{array}{c}0.28 \\
124.574\end{array}$ & $\begin{array}{c}-4.10 \\
120.908\end{array}$ & $\begin{array}{c}0.60 \\
203.587\end{array}$ & $\begin{array}{l}-1.43 \\
400.156\end{array}$ & $\begin{array}{c}-0.58 \\
2001.464\end{array}$ & $\begin{array}{c}0.24 \\
12534.472\end{array}$ & 二 & $2742 \overline{24} .481$ & 二 & $93 \overline{7.989}$ & $\begin{array}{l}-0.68 \\
47.311\end{array}$ & $\begin{array}{l}-0.13 \\
30.499\end{array}$ & $\begin{array}{l}-0.01 \\
10.281\end{array}$ & $\begin{array}{l}-0.09 \\
0.440\end{array}$ \\
\hline \multirow{2}{*}{\multicolumn{18}{|c|}{ Algoritmo 5 e CONOPT }} \\
\hline & $1^{*}$ & & 1 & 2 & 6 & 6 & $\overline{17}$ & & 26 & 27 & 38 & 52 & 58 & 59 & & 61 & 63 \\
\hline Perdas [MV & 132.29 & 132.29 & 132.13 & 131.29 & 128.53 & 128.53 & 124.55 & 124.5 & 121.19 & 120.6 & 116.48 & 110. & 106.39 & 106.2 & 106.2 & 106.17 & 106.15 \\
\hline$\Delta$ & & 0 & & 1 & 4 & 0 & 1 & 0 & 9 & 1 & 11 & 1 & 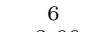 & 1 & 1 & 1 & 2 \\
\hline$\Delta$ & - & 0 & -0.16 & -0.84 & -2 & 0 & -3. & 0 & -3.36 & -0.53 & -4 & -18.48 & -3.66 & -0.13 & -0 & -0.04 & -0.02 \\
\hline 5 & 3 & 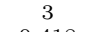 & 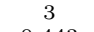 & 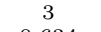 & r & 3 & 5 & (5 & 5 & 3 & 3 & ( & $=$ & 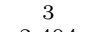 & 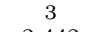 & 3 & 3 \\
\hline mpo [s] & 0.410 & 0.418 & 0.443 & 0.634 & 0.958 & 2.352 & 1.383 & 0.898 & 0.977 & 1.148 & 1.224 & 1.788 & 3.518 & 2.404 & 2.442 & 2.044 & 1.817 \\
\hline
\end{tabular}

Tabela 6.24 - Resultados do sistema IEEE de 300 barras para os diferentes valores de $\omega$. Perdas são dadas em MW e o tempo em segundos.

\begin{tabular}{c|c|c|c|c|c|c|c|c|c|c|c|c|c}
\hline$\omega$ & 0.0 & 0.1 & 0.2 & 0.3 & 0.4 & 0.5 & 0.6 & 0.7 & 0.8 & 0.9 & 1.0 \\
\hline \multicolumn{10}{c|}{ Algoritmo 5 e CONOPT } \\
\hline $\mathrm{N}$ & 4 & 6 & 20 & 38 & 48 & 68 & 77 & 87 & 95 & 110 & 168 \\
Perdas [MW] & 405.08 & 394.63 & 378.10 & 360.70 & 355.50 & 347.69 & 345.69 & 344.03 & 343.43 & 342.77 & 342.50 \\
$\Delta \mathrm{N}$ & - & 2 & 14 & 18 & 10 & 20 & 9 & 10 & 8 & 15 & 58 \\
$\Delta \mathrm{P}[\mathrm{MW}]$ & - & -10.45 & -16.53 & -17.40 & -5.20 & -7.81 & -2.00 & -1.66 & -0.60 & -0.66 & -0.27 \\
Iterações & 5 & 3 & 3 & 3 & 3 & 3 & 3 & 3 & 3 & 3 & 3 \\
Tempo [s] & 0.983 & 1.155 & 1.504 & 2.376 & 3.549 & 4.622 & 4.614 & 5.418 & 8.660 & 7.956 & 8.181 \\
\hline
\end{tabular}


As Figuras 6.15, 6.16, 6.17 e 6.18 apresentam as perdas ativas pelo número de ações de controle, encontrados pelos solvers DICOPT e KNITRO para o problema (5.2) e pelo Algoritmo 5 juntamente com CONOPT para o problema modificado (5.3), para os sistemas IEEE de 14, 30, 57 e 118 barras, respectivamente.

A Figura 6.19 apresenta as perdas ativas pelo número de ações de controle encontrados para o sistema IEEE de 300 barras como modelo (5.3), quando utilizamos a função sigmoidal para o tratamento das variáveis binárias (Algoritmo 5) e o solver CONOPT. A Figura 6.20 apresenta o número de ações de controle, enquanto a Figura 6.21 apresenta as perdas ativas para os diferentes valores de $\omega$.

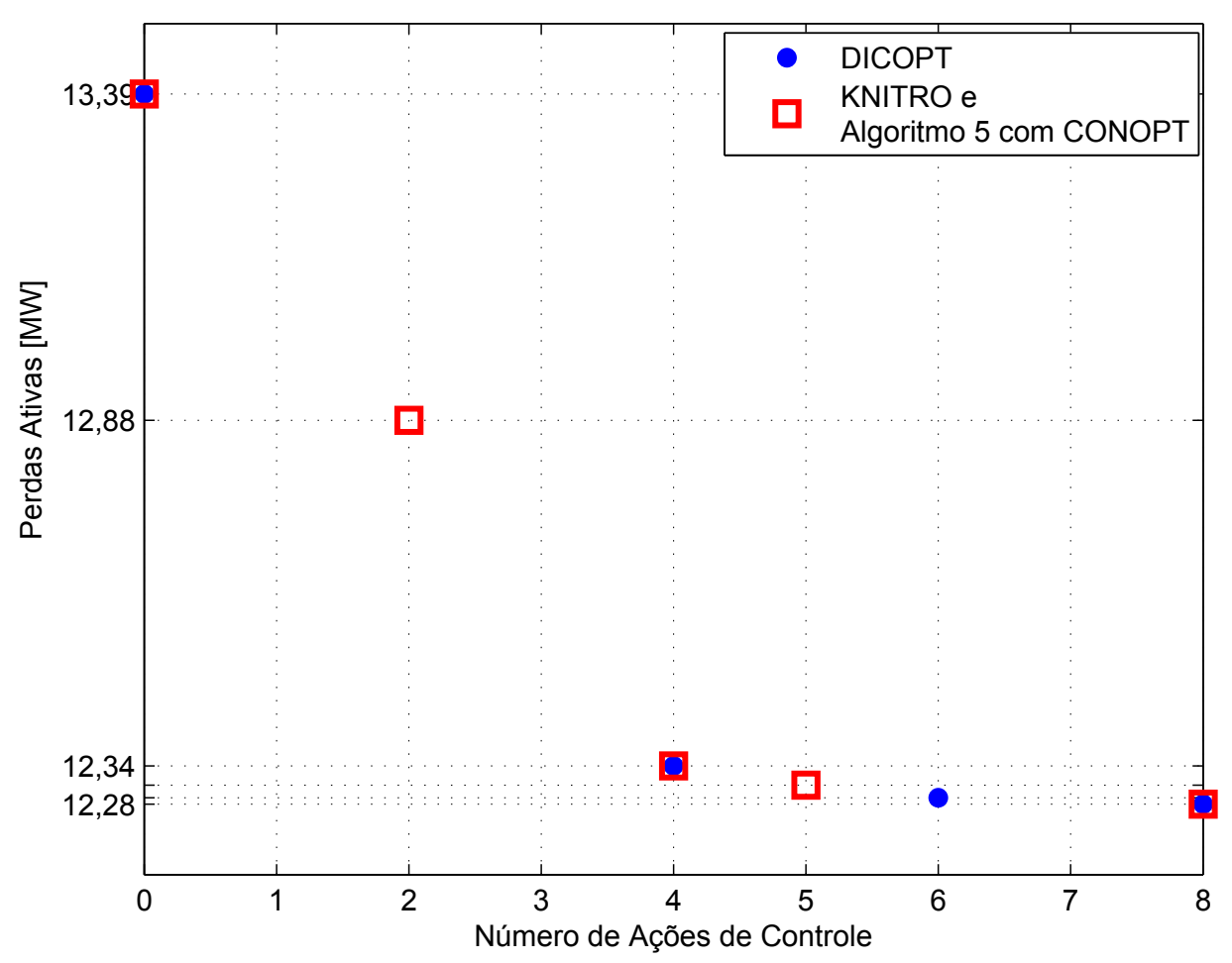

Figura 6.15 - Perdas ativas pelo número de ações de controle para o sistema IEEE de 14 barras (Método da Soma Ponderada). 


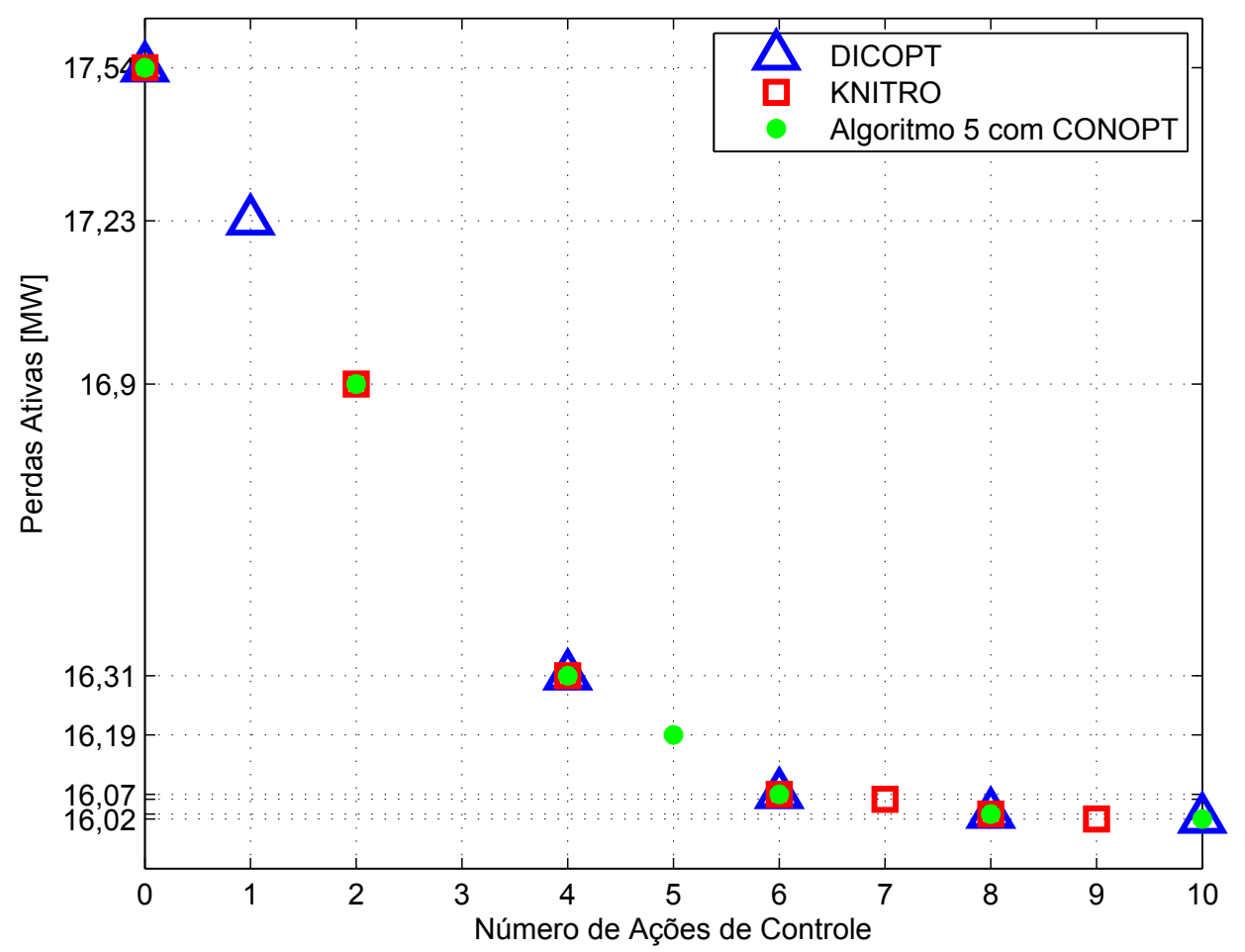

Figura 6.16 - Perdas ativas pelo número de ações de controle para o sistema IEEE de 30 barras (Método da Soma Ponderada).

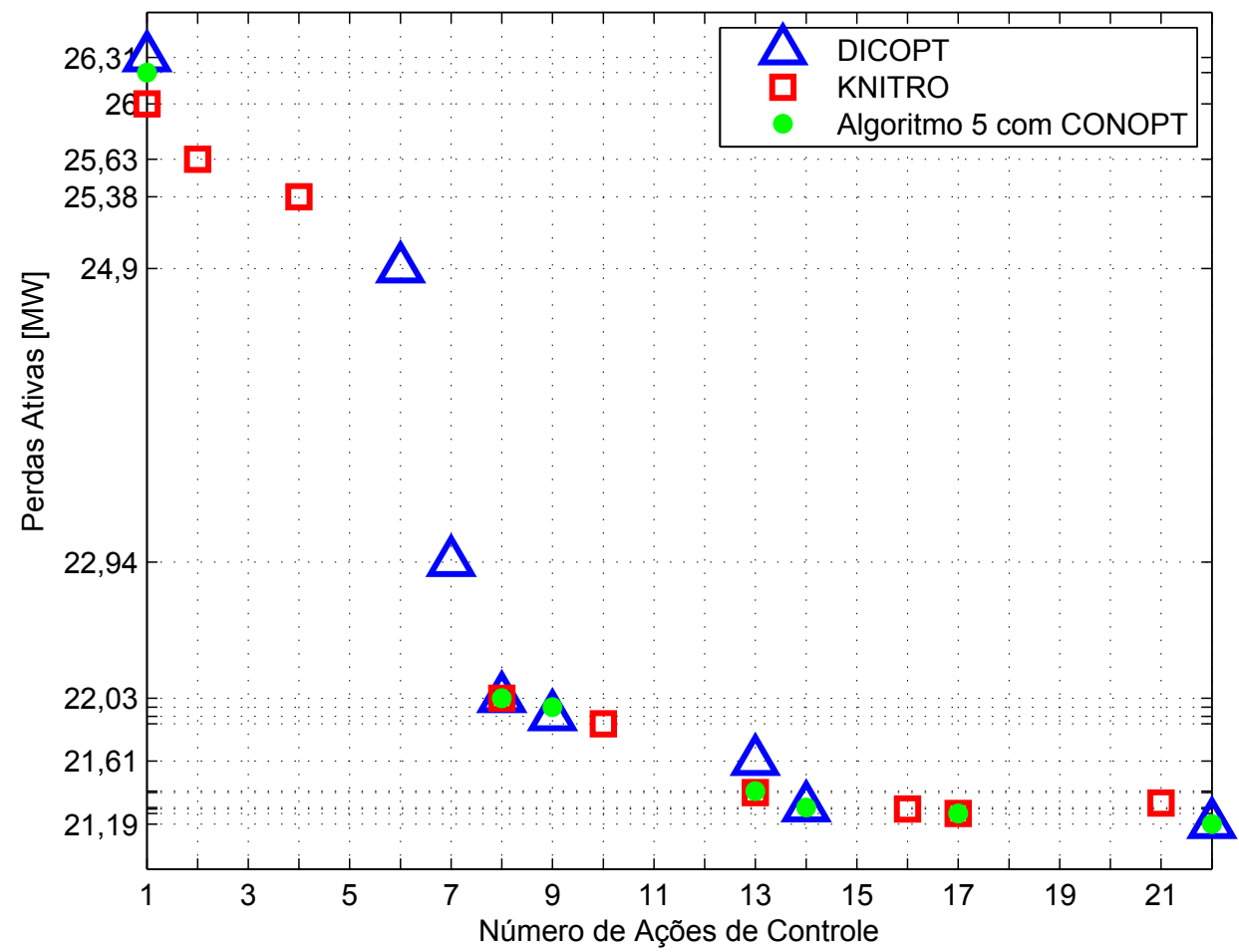

Figura 6.17 - Perdas ativas pelo número de ações de controle para o sistema IEEE de 57 barras (Método da Soma Ponderada). 


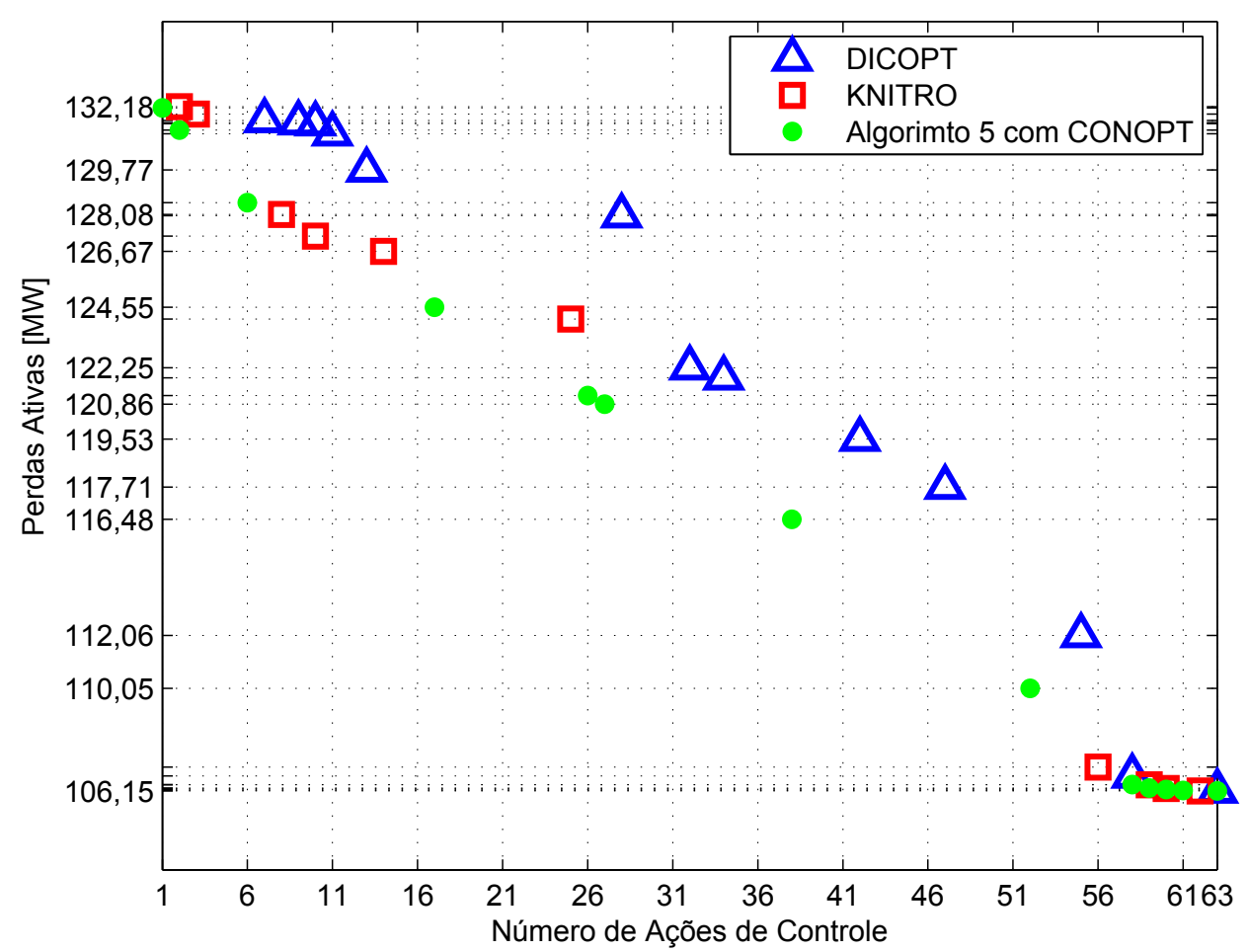

Figura 6.18 - Perdas ativas pelo número de ações de controle para o sistema IEEE de 118 barras (Método da Soma Ponderada).

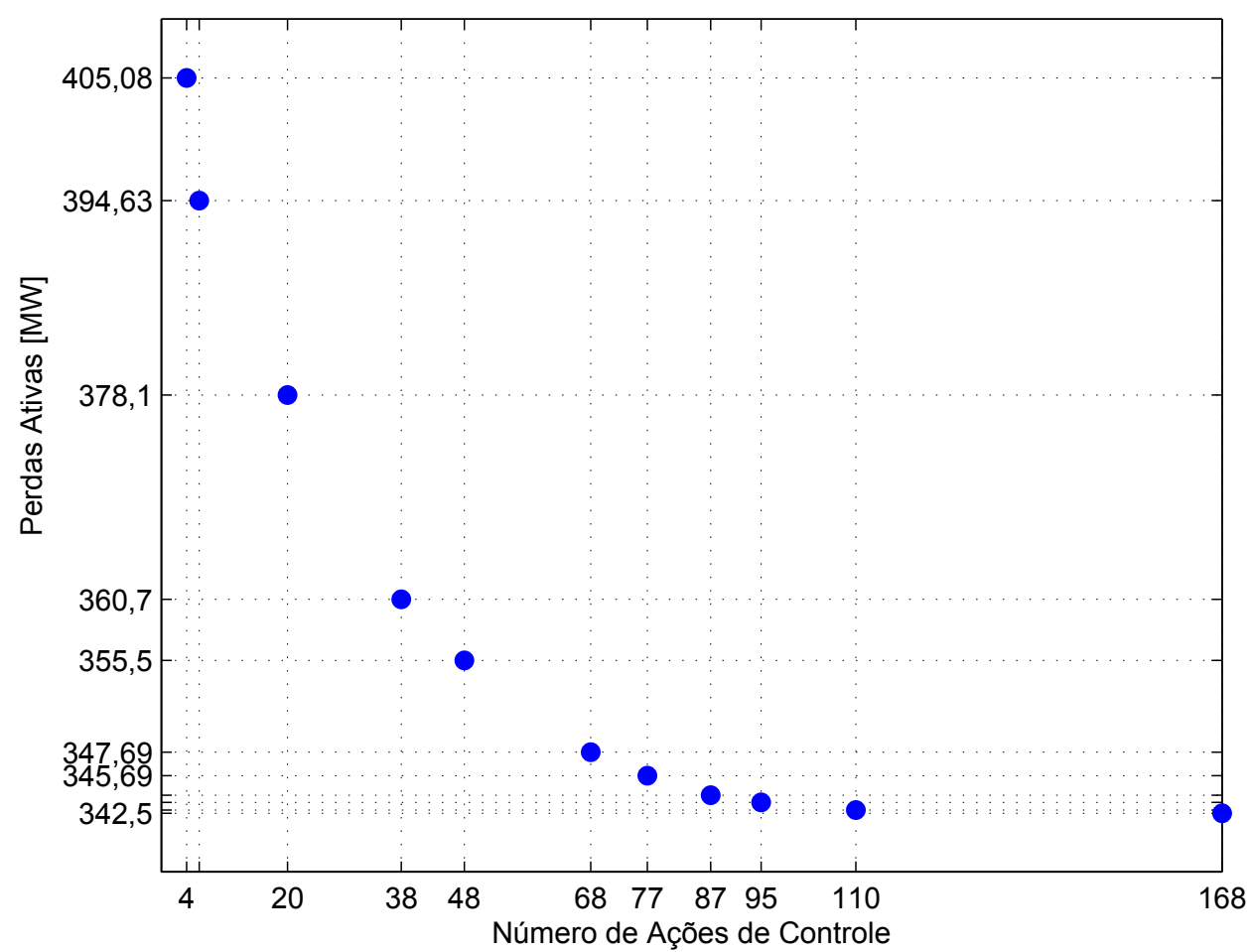

Figura 6.19 - Perdas ativas pelo número de ações de controle pelo solver CONOPT e a função sigmoidal para o tratamento das variáveis binárias para o sistema IEEE de 300 barras (Método da Soma Ponderada). 


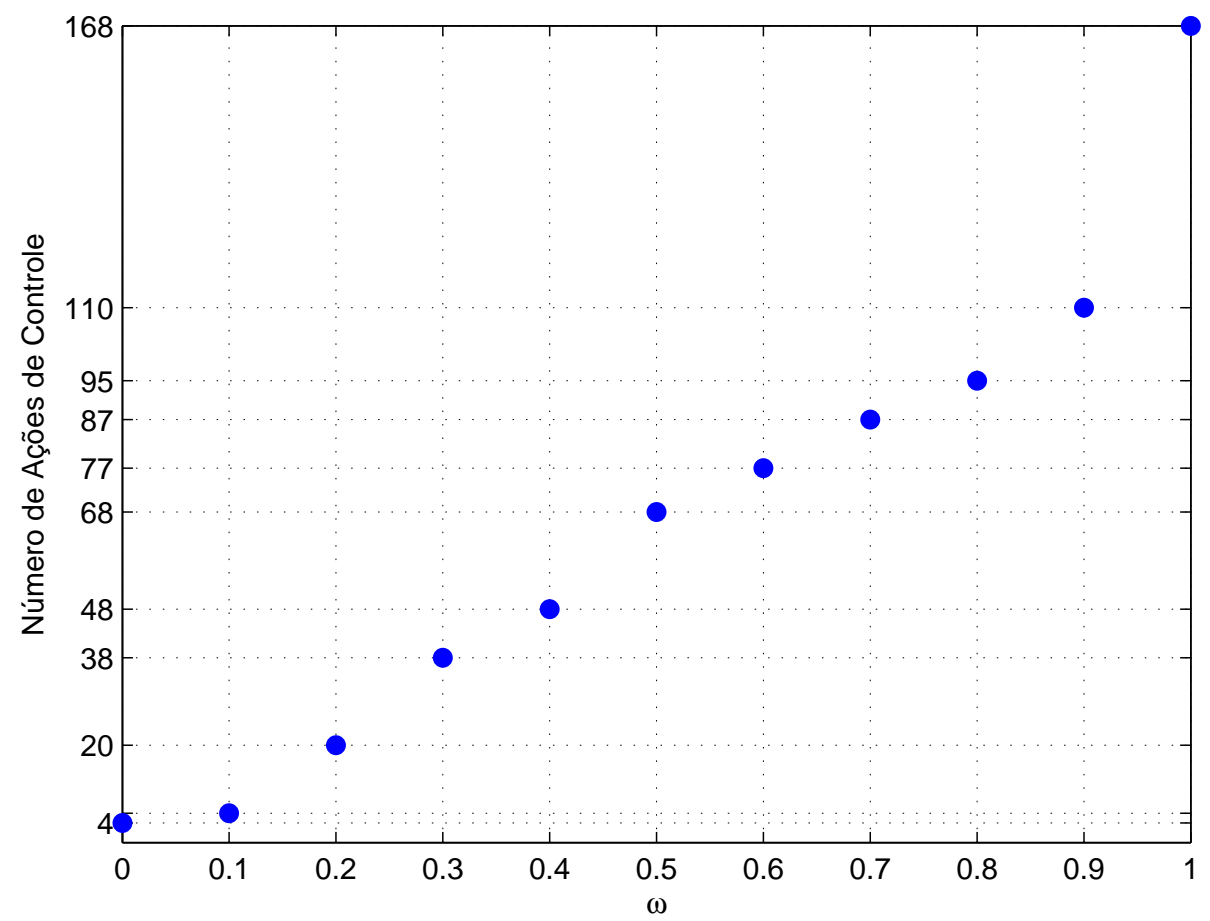

Figura 6.20 - Ações de Controle $\times \omega$ (Método da Soma Ponderada).

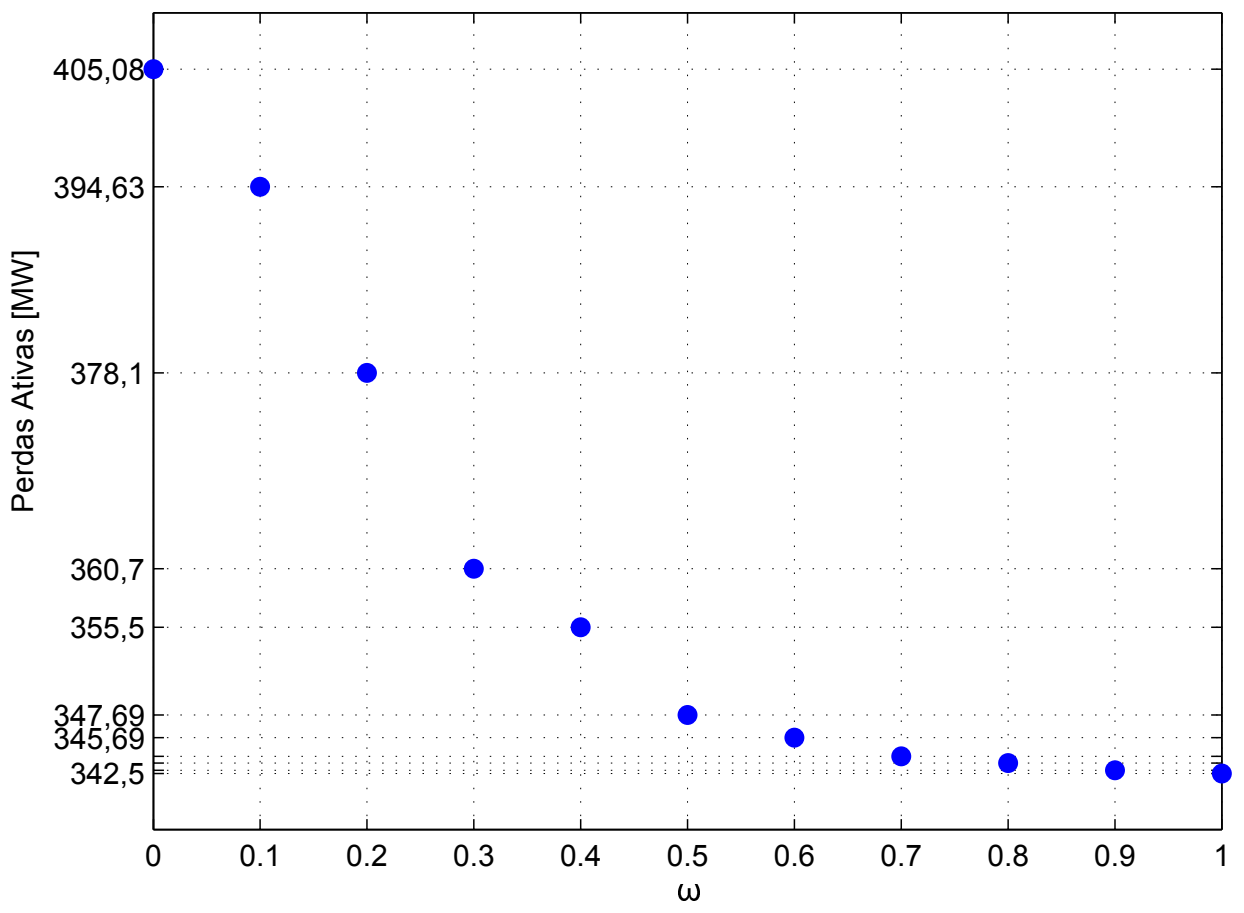

Figura 6.21 - Perdas Ativas $\times \omega$ (Método da Soma Ponderada). 


\section{- Análise dos Resultados Obtidos}

Foram comparadas as soluções encontradas pelos solvers DICOPT e KNITRO para o problema (5.2) com as soluções obtidas pelo Algoritmo 5 e CONOPT para o problema modificado (5.3), que utiliza a função sigmoidal no tratamento das variáveis binárias. Ao comparar os resultados para cada sistema, observamos que as soluções encontradas para os sistemas de 14 e 30 barras são equivalentes para todos os solvers. Para o sistema de 57 barras, DICOPT encontrou oito soluções não dominadas, KNITRO encontrou nove e o Algoritmo 5 com CONOPT encontrou sete. Comparando ponto-a-ponto e removendo soluções dominadas entre os solvers, DICOPT tem três soluções na curva combinada, KNITRO tem seis e o Algoritmo 5 com CONOPT tem quatro. Este efeito pode ser visto na Figura 6.22.

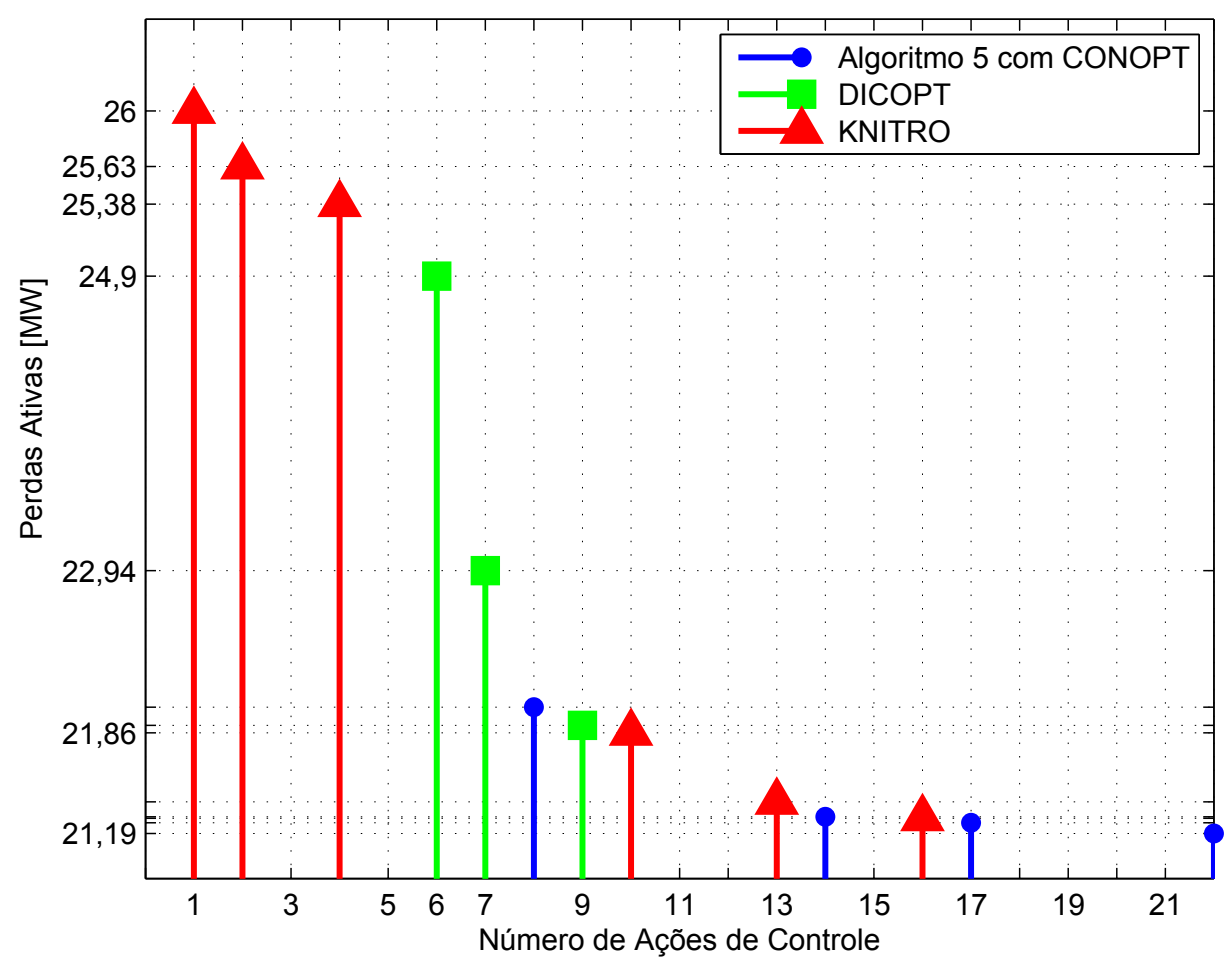

Figura 6.22 - Perdas ativas pelo número de ações de controle da curva combinada do sistema de 57 barras. Soluções não dominadas geradas pela combinação das soluções dos três solvers (Método da Soma Ponderada).

Para o sistema IEEE de 118 barras, notamos que entre os pesos 0.4 e 0.5 existe um gap muito grande no número de ações de controle. Para o solver DICOPT existe um gap de 25 ações de controle entre 0.4 e 0.5 , para o solver KNITRO o peso 0.5 não gera uma solução factível, e ao comparar o intervalo entre $\omega=0.4$ e $\omega=0.6$, o gap é de 48 posições no número de ações de controle. Para o Algoritmo 5 e CONOPT o gap entre os pesos 0.4 e 0.5 também é grande, possuindo 46 posições. O sistema IEEE de 118 barras possui num total 63 ações de controle, um gap de 48 posições é aproximadamente $76 \%$ do total das ações de controle. Com isso foi necessário realizar testes com pesos $\omega$ no intervalo $[0.4,0.5]$. 
Comparando ponto-a-ponto entre os solvers e removendo soluções dominadas, DICOPT não tem soluções na curva combinada, KNITRO tem seis soluções na curva combinada e CONOPT tem doze soluções na curva combinada. Este efeito pode ser visto na Figura 6.23.

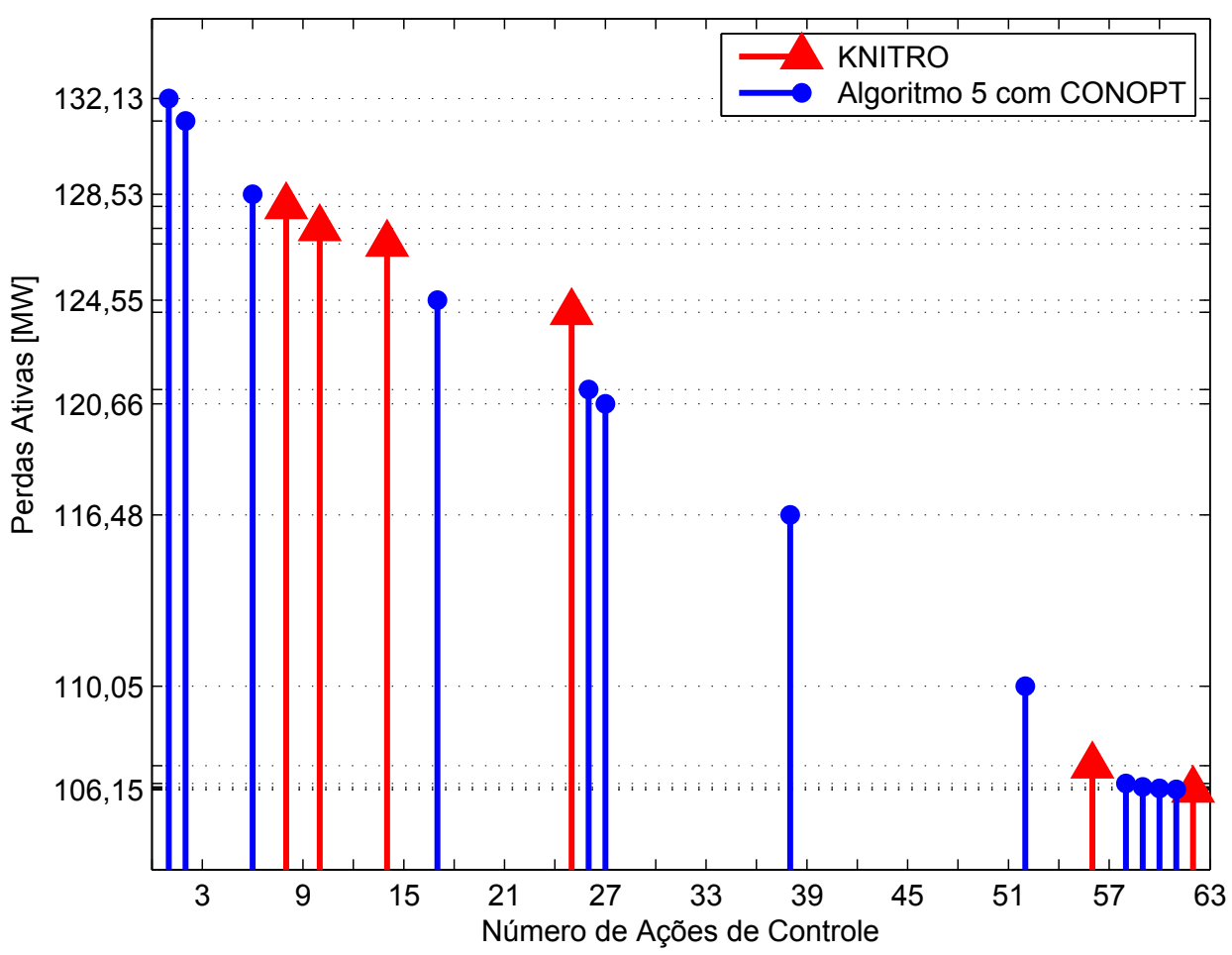

Figura 6.23 - Perdas ativas pelo número de ações de controle da curva combinada do sistema de 118 barras. Soluções não dominadas geradas pela combinação das soluções dos três solvers (Método da Soma Ponderada).

A dificuldade em resolver o problema (5.2) com variáveis binárias pode ser observada a através dos resultados encontrados com os solvers DICOPT e KNITRO, conforme a quantidade das variáveis binárias aumentam. Por exemplo, para os sistemas de 14 e 30 barras, DICOPT e KNITRO resolvem o problema (5.2) e encontram uma solução factível para todos os pesos $\omega$ estabelecidos, além disso o resultado desses dois solvers é similar com os resultados encontrados para o Algoritmo 5 com CONOPT (problema (5.3)). Para o sistema de 57 barras, KNITRO domina mais soluções do que o Algoritmo 5 com CONOPT, e estes dois dominam mais soluções que DICOPT. O tempo de processamento computacional é bem próximo. Para o sistema de 118 barras, DICOPT não encontra a melhor solução possível, parando o processo por deterioramento do problema, já KNITRO consegue encontrar sua melhor solução para 15 dos 17 pesos estabelecidos. O Algoritmo 5 com CONOPT encontra soluções para todos os pesos, e estas são melhores do que as encontradas por KNITRO. Alem disso, o tempo total para encontrar soluções para os 17 pesos é de 24.858 segundos para o Algoritmo 5 com CONOPT (problema (5.3)), DICOPT utiliza aproximadamente 1 hora para encontrar todas as soluções do problema (5.2) e KNITRO utiliza aproximadamente 12 horas para encontrar 15 das 17 soluções do problema (5.2). 
Quando testamos o sistema de 300 barras, que possui 176 variáveis binárias, observamos que o DICOPT falha em fornecer soluções para os pesos testados, já o KNITRO encontra soluções para apenas 3 dos 11 casos e utiliza um tempo acima de 156 horas. A Tabela 6.25 apresenta os resultados obtidos para o sistema de 300 barras com o solver KNITRO.

Tabela 6.25 - Número de ações de controle e perdas ativas para o sistema de 300 barras usando KNITRO.

\begin{tabular}{c|c|c|c}
\hline$\omega$ & 0.0 & 0.1 & 0.8 \\
\hline $\mathrm{N}$ & 1 & 3 & 101 \\
Losses [MW] & 406.56 & 401.40 & 343.14 \\
Time [s] & 626.242 & 806.751 & 560502.522 \\
\hline
\end{tabular}

Comparando ponto-a-ponto e removendo soluções dominadas entre os solvers KNITRO e CONOPT obtemos a Figura 6.24.

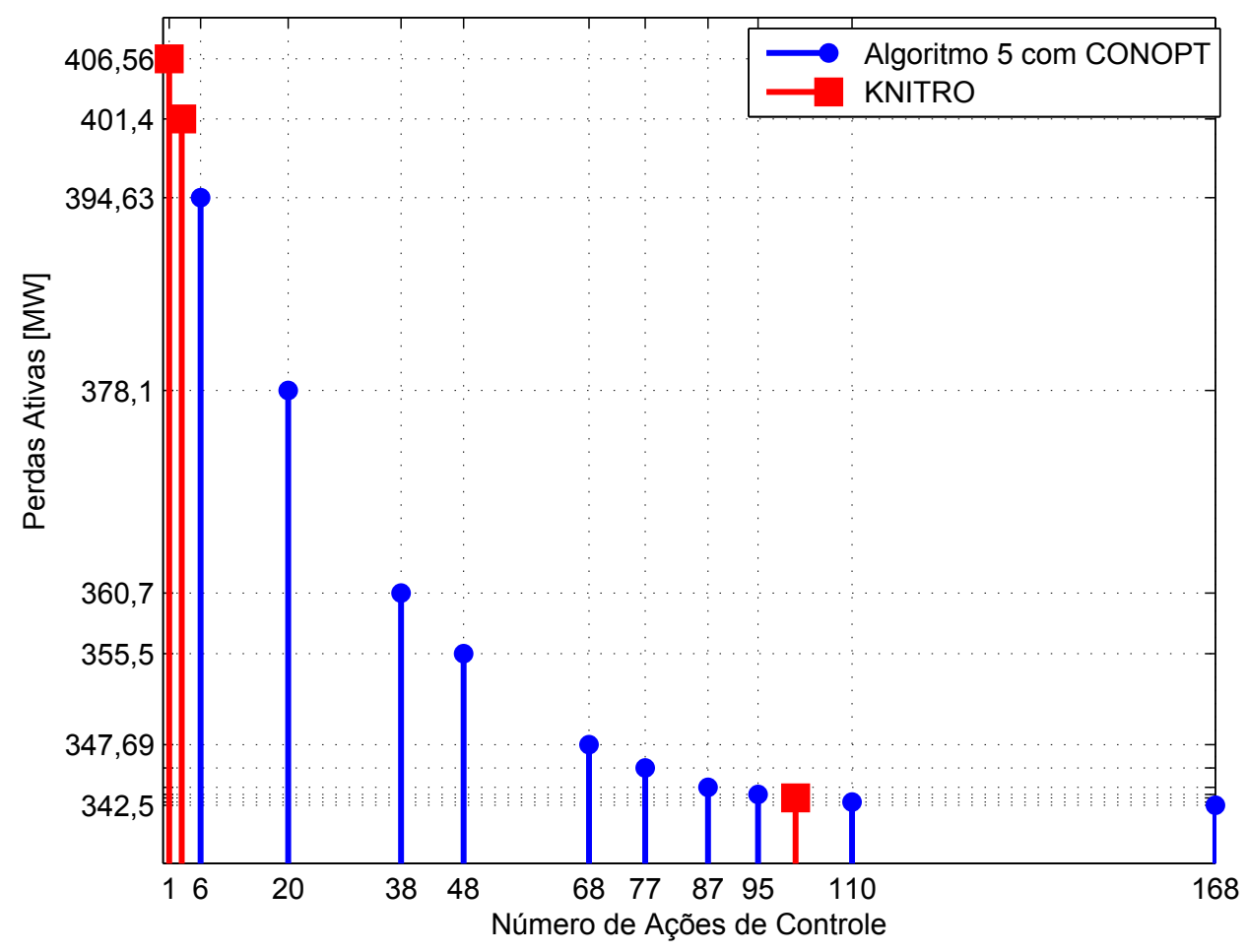

Figura 6.24 - Perdas ativas pelo número de ações de controle da curva combinada do sistema de 300 barras. Soluções não dominadas geradas pela combinação das soluções de KNITRO e CONOPT (Método da Soma Ponderada). 


\subsubsection{Método $\epsilon$-Restrito}

O modelo de FPO Multiobjetivo com taps de transformadores contínuos e os bancos de capacitores e reatores shunts fixos (2.14) é modificado pelo método $\epsilon$-Restrito como em (5.5). Utiliza-se a função Quadrática (3.5) para a modelagem das variáveis binárias do problema (5.5) como em (5.6). O solver IPOPT é utilizado na resolução do modelo (5.6). Para verificar a eficácia desta estratégia utilizando a função quadrática na modelagem das variáveis binárias aplica-se os solvers DICOPT e KNITRO para tratar as variáveis binárias do problema (5.5).

Os testes foram realizados com diferentes valores de $\epsilon$ para cada sistema. Note que a solução viável que apresenta um trade-off entre os objetivos, para o modelo (5.6), não pode ser chamada de uma solução ótima de Pareto. Isso acontece pois resolve-se um problema modificado, e não, o original. Encontramos uma solução para o problema modificado que é uma solução viável para o problema original.

A eficiência desta estratégia é altamente afetada pela inicialização dos parâmetros $s_{1} \mathrm{e}$ $s_{2}$. Realizamos testes com sete pares diferentes para estes parâmetros, que são apresentados na Tabela 6.26.

Tabela 6.26 - Inicialização das Variáveis $s_{1}$ e $s_{2}$

\begin{tabular}{c|ccccccc}
\hline & $\mathrm{A}$ & $\mathrm{B}$ & $\mathrm{C}$ & $\mathrm{D}$ & $\mathrm{E}$ & $\mathrm{F}$ & $\mathrm{G}$ \\
\hline$s_{1}$ & 0 & 0 & 0.5 & 1 & 0.5 & 1 & 1 \\
$s_{2}$ & 0 & 0.5 & 0 & 0 & 0.5 & 0.5 & 1 \\
\hline
\end{tabular}

Os resultados apresentados a seguir são os melhores encontrados comparando os sete casos de entrada dos parâmetros. $\Delta \mathrm{P}$ é a diferença entre as perdas ativas para o $\epsilon_{i}$ atual e para o anterior $\epsilon_{i-1}$. Matematicamente, $\Delta \mathrm{P}$ pode ser calculado como:

$$
\Delta \mathrm{P}=\mathrm{P}^{\left(\epsilon_{i}\right)}-\mathrm{P}^{\left(\epsilon_{i-1}\right)}
$$

onde $\mathrm{P}^{\left(\epsilon_{i}\right)}$ é a perda ativa na solução corrente encontrada para $\epsilon_{i}$. Devemos estar cientes de que o método $\epsilon$-Restrito não garante que todos os valores de $\epsilon$ escolhidos geram uma solução factível para o problema.

As Tabelas 6.27, 6.28, 6.29, 6.30 e 6.31 apresentam os resultados obtidos para os sistemas IEEE de 14, 30, 57, 118 e 300 barras, respectivamente. As Figuras 6.25, 6.26, 6.27, 6.28 e 6.29 apresentam as perdas ativas pelo número de ações de controle encontrados para o modelo (5.5) com os solvers DICOPT e KNITRO, e para o modelo (5.6) com o solver IPOPT, nos sistemas IEEE de 14, 30, 57, 118 e 300 barras, respectivamente. 
Tabela 6.27 - Resultados para o sistema IEEE de 14 barras com as diferentes estratégias. Soluções com * são dominadas pelas demais.

\begin{tabular}{|c|c|c|c|c|c|c|c|c|c|}
\hline \multicolumn{10}{|c|}{ DICOPT } \\
\hline $\bar{\epsilon}$ & 0 & 1 & 2 & 3 & 4 & 5 & $6^{*}$ & 7 & 8 \\
\hline Perdas [MW] & 13.39 & 13.17 & 12.88 & 12.57 & 12.34 & 12.31 & 12.40 & 12.29 & 12.28 \\
\hline$\Delta \mathrm{P}[\mathrm{MW}]$ & - & -0.22 & -0.29 & -0.31 & -0.23 & -0.03 & 0.09 & -0.11 & -0.01 \\
\hline$\sum_{k \in \mathcal{B}} s_{1_{k}}$ & 0 & 1 & 2 & 3 & 3 & 3 & 5 & 5 & 5 \\
\hline$\sum_{k, m \in \mathcal{T}} s_{2_{k m}}$ & 0 & 0 & 0 & 0 & 1 & 2 & 1 & 2 & 3 \\
\hline Tempo $[\mathrm{s}]$ & 0.194 & 0.141 & 0.778 & 0.205 & 0.180 & 0.318 & 0.146 & 0.289 & 0.083 \\
\hline \multicolumn{10}{|c|}{ KNITRO } \\
\hline $\bar{\epsilon}$ & 0 & 1 & 2 & 3 & 4 & 5 & 6 & $7^{*}$ & 8 \\
\hline Perdas [MW] & 13.39 & 13.17 & 12.88 & 12.57 & 12.42 & 12.40 & 12.39 & 13.06 & 12.28 \\
\hline$\Delta \mathrm{P}[\mathrm{MW}]$ & - & -0.22 & -0.29 & -0.31 & -0.15 & -0.02 & -0.01 & 0.67 & -0.78 \\
\hline$\sum_{k \in \mathcal{B}} s_{1_{k}}$ & 0 & 1 & 2 & 3 & 4 & 5 & 4 & 4 & 5 \\
\hline$\sum_{k, m \in \mathcal{T}} s_{2}{ }_{k m}$ & 0 & 0 & 0 & 0 & 0 & 0 & 2 & 3 & 3 \\
\hline Tempo $[\mathrm{s}]$ & 0.228 & 0.112 & 0.113 & 0.261 & 0.117 & 0.160 & 0.160 & 0.099 & 0.246 \\
\hline \multicolumn{10}{|c|}{ IPOPT } \\
\hline $\bar{\epsilon}$ & 0 & 1 & 2 & 3 & 4 & 5 & 6 & $7^{*}$ & 8 \\
\hline Perdas [MW] & 13.39 & 13.38 & 13.16 & 13.15 & 12.77 & 12.40 & 12.29 & 12.29 & 12.28 \\
\hline$\Delta \mathrm{P}[\mathrm{MW}]$ & - & -0.01 & -0.22 & -0.01 & -0.38 & -0.37 & -0.11 & 0.00 & -0.01 \\
\hline$\sum_{k \in \mathcal{B}} s_{1_{k}}$ & 0 & 1 & 2 & 3 & 3 & 5 & 5 & 4 & 5 \\
\hline$\sum_{k, m \in \mathcal{T}} s_{2_{k m}}$ & 0 & 0 & 0 & 0 & 1 & 0 & 1 & 3 & 3 \\
\hline$s_{1}^{(0)}$ & 1 & 1 & 1 & 1 & 1 & 1 & 1 & 1 & 1 \\
\hline$s_{2}^{(0)}$ & 0.5 & 0 & 0.5 & 0.5 & 1 & 0.5 & 1 & 0.5 & 0.5 \\
\hline Tempo $[\mathrm{s}]$ & 0.415 & 0.260 & 0.286 & 0.323 & 0.138 & 0.254 & 0.267 & 0.282 & 0.120 \\
\hline
\end{tabular}

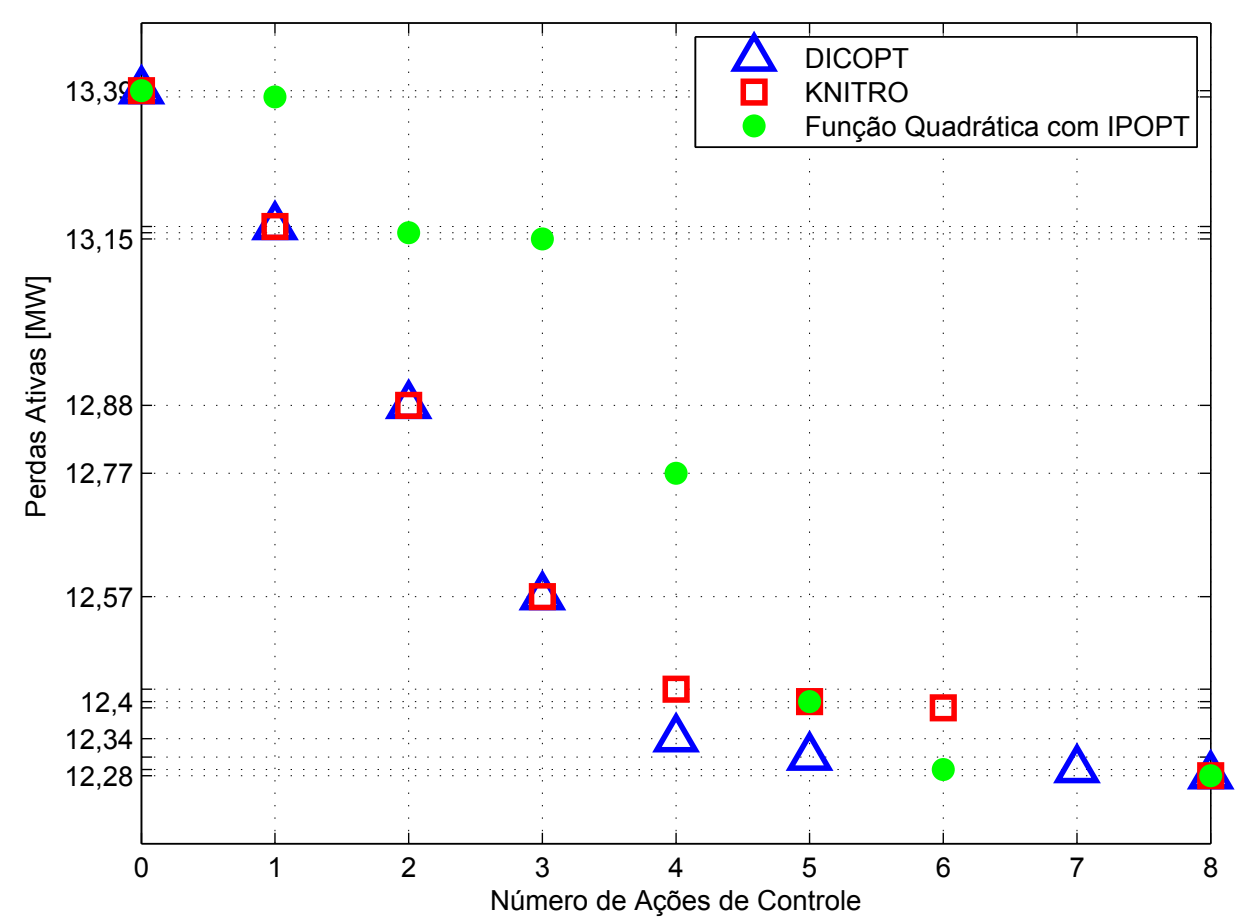

Figura 6.25 - Perdas ativas pelo número de ações de controle para o sistema de 14 barras com todos os solvers (Método $\epsilon$-Restrito). 
Tabela 6.28 - Resultados para o sistema IEEE de 30 barras com as diferentes estratégias. Soluções com * são dominadas pelas demais.

\begin{tabular}{|c|c|c|c|c|c|c|c|c|c|c|c|}
\hline \multicolumn{12}{|c|}{ DICOPT } \\
\hline $\bar{\epsilon}$ & 0 & 1 & 2 & 3 & 4 & 5 & 6 & 7 & 8 & 9* & 10 \\
\hline Perdas [MW] & 17.54 & 17.23 & 16.90 & 16.62 & 16.31 & 16.19 & 16.07 & 16.04 & 16.03 & 16.03 & 16.02 \\
\hline$\Delta \mathrm{P}[\mathrm{MW}]$ & - & -0.31 & -0.33 & -0.28 & -0.31 & -0.12 & -0.12 & -0.03 & -0.01 & 0.00 & -0.01 \\
\hline$\sum_{k \in \mathcal{B}} s_{1_{k}}$ & 0 & 1 & 2 & 3 & 4 & 4 & 5 & 5 & 6 & 5 & 6 \\
\hline$\sum_{k, m \in \mathcal{T}} s_{2_{k m}}$ & 0 & 0 & 0 & 0 & 0 & 1 & 1 & 2 & 2 & 4 & 4 \\
\hline Tempo $[\mathrm{s}]$ & 0.184 & 0.384 & 0.573 & 0.467 & 0.473 & 0.612 & 0.722 & 0.582 & 0.586 & 0.171 & 0.195 \\
\hline \multicolumn{12}{|c|}{ KNITRO } \\
\hline$\epsilon$ & 0 & 1 & 2 & 3 & 4 & 5 & 6 & 7 & 8 & $9 *$ & 10 \\
\hline Perdas [MW] & 17.54 & 17.23 & 16.92 & 16.62 & 16.31 & 16.19 & 16.07 & 16.04 & 16.03 & 16.99 & 16.02 \\
\hline$\Delta \mathrm{P}[\mathrm{MW}]$ & - & -0.31 & -0.31 & -0.30 & -0.31 & -0.12 & -0.12 & -0.03 & -0.01 & 0.96 & -0.97 \\
\hline$\sum_{k \in \mathcal{B}} s_{1_{k}}$ & 0 & 1 & 2 & 3 & 4 & 4 & 5 & 5 & 6 & 5 & 6 \\
\hline$\sum_{k, m \in \mathcal{T}} s_{2 k m}$ & 0 & 0 & 0 & 0 & 0 & 1 & 1 & 2 & 2 & 4 & 4 \\
\hline Tempo $[\mathrm{s}]$ & 0.238 & 0.216 & 0.343 & 0.282 & 0.284 & 0.307 & 0.328 & 0.344 & 0.333 & 0.253 & 0.202 \\
\hline \multicolumn{12}{|c|}{ IPOPT } \\
\hline$\epsilon$ & 0 & 1 & 2 & 3 & $4^{*}$ & 5 & 6 & 7 & 8 & 9 & 10 \\
\hline Perdas [MW] & 17.54 & 17.46 & 17.44 & 17.16 & 17.17 & 16.77 & 16.16 & 16.06 & 16.04 & 16.03 & 16.02 \\
\hline$\Delta \mathrm{P}[\mathrm{MW}]$ & - & -0.08 & -0.02 & -0.28 & 0.01 & -0.40 & -0.10 & -0.02 & -0.02 & -0.01 & -0.01 \\
\hline$\sum_{k \in \mathcal{B}} s_{1_{k}}$ & 0 & 0 & 0 & 3 & 4 & 5 & 6 & 6 & 6 & 6 & 6 \\
\hline$\sum_{k, m \in \mathcal{T}} s_{2_{k m}}$ & 0 & 1 & 2 & 0 & 0 & 0 & 0 & 1 & 2 & 3 & 4 \\
\hline$s_{1}^{(0)}$ & 1 & 1 & 0 & 1 & 1 & 1 & 1 & 1 & 1 & 1 & 1 \\
\hline$s_{2}^{(0)}$ & 0.5 & 1 & 0.5 & 0.5 & 1 & 0.5 & 0.5 & 0.5 & 0.5 & 0 & 0.5 \\
\hline Tempo [s] & 0.275 & 0.403 & 0.255 & 0.301 & 0.407 & 0.329 & 0.357 & 0.271 & 0.190 & 0.349 & 0.255 \\
\hline
\end{tabular}

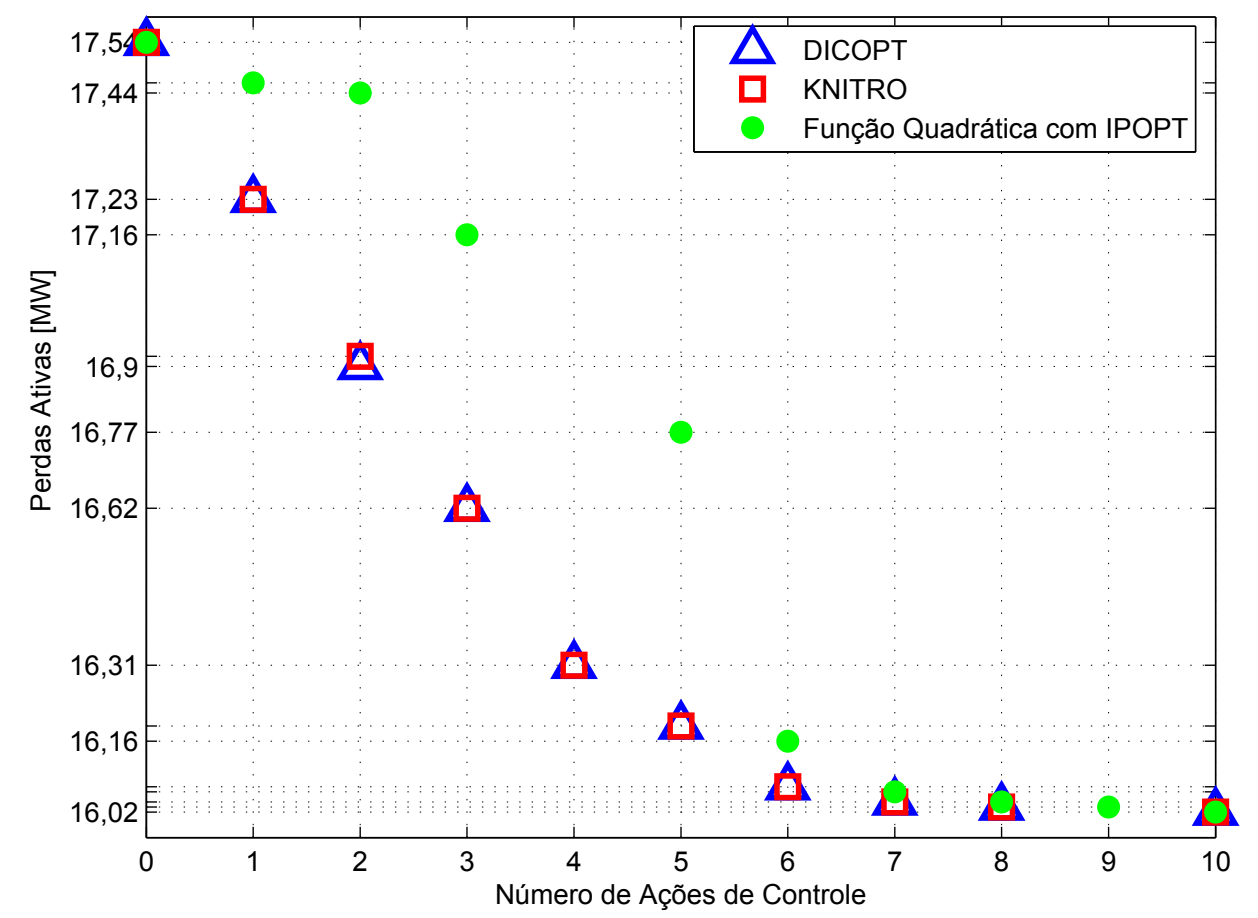

Figura 6.26 - Perdas ativas pelo número de ações de controle para o sistema de 30 barras com todos os solvers (Método $\epsilon$-Restrito). 
Tabela 6.29 - Resultados para o sistema IEEE de 57 barras. Soluções com * são dominadas pelas demais.

\begin{tabular}{|c|c|c|c|c|c|c|c|c|c|c|c|c|c|c|c|c|c|c|c|c|c|c|}
\hline \multicolumn{23}{|c|}{ DICOPT } \\
\hline $\bar{\epsilon}$ & 1 & 2 & $3^{*}$ & 4 & $5^{*}$ & 6 & 7 & 8 & 9 & 10 & $11^{*}$ & $12^{*}$ & 13 & 14 & 15 & $16^{*}$ & 17 & 18 & 19 & 20 & $21^{*}$ & 22 \\
\hline Perdas $[\mathrm{MW}]$ & 26.00 & 25.95 & 25.99 & 24.95 & 25.27 & 23.20 & 22.94 & 22.03 & 21.91 & 21.84 & 21.92 & 21.90 & 21.40 & 21.35 & 21.27 & 21.28 & 21.26 & 21.23 & 21.22 & 21.20 & 21.20 & 21.19 \\
\hline$\Delta \mathrm{P}[\mathrm{MW}]$ & - & -0.05 & 0.04 & -1.04 & 0.32 & -2.07 & -0.26 & -0.91 & -0.12 & -0.07 & 0.08 & -0.02 & -0.50 & -0.05 & -0.08 & 0.01 & -0.02 & -0.03 & -0.01 & -0.02 & 0.00 & -0.01 \\
\hline$\sum_{k \in \mathcal{R}} s_{1_{k}}$ & 1 & 2 & 2 & 4 & 5 & 6 & 6 & 7 & 7 & 7 & 7 & 7 & 7 & 7 & 7 & 7 & 7 & 7 & 7 & 7 & 7 & 7 \\
\hline$\sum_{k, m \in \mathcal{T}} s_{2}{ }_{k m}$ & 0 & 0 & 1 & 0 & 0 & 0 & 1 & 1 & 2 & 3 & 4 & 5 & 6 & 7 & 8 & 9 & 10 & 11 & 12 & 13 & 14 & 15 \\
\hline Tempo $[\mathrm{s}]$ & 0.803 & 2.892 & 0.718 & 0.975 & 0.441 & 0.824 & 0.899 & 0.751 & 1.741 & 1.309 & 1.385 & 1.728 & 3.790 & 3.562 & 1.961 & 2.678 & 2.102 & 1.468 & 1.290 & 0.608 & 0.866 & 0.285 \\
\hline \multicolumn{23}{|c|}{ KNITRO } \\
\hline $\bar{\epsilon}$ & 1 & $2^{*}$ & 3 & 4 & $5^{*}$ & 6 & 7 & 8 & 9 & 10 & 11 & 12 & 13 & 14 & 15 & 16 & 17 & 18 & 19 & 20 & $21^{*}$ & 22 \\
\hline Perdas $[\mathrm{MW}]$ & 26.00 & 26.21 & 25.55 & 25.39 & 25.74 & 24.07 & 23.13 & 22.03 & 21.94 & 21.77 & 21.61 & 21.53 & 21.40 & 21.36 & 21.29 & 21.28 & 21.24 & 21.23 & 21.21 & 21.20 & 24.19 & 21.19 \\
\hline$\Delta \mathrm{P}[\mathrm{MW}]$ & - & 0.21 & -0.66 & -0.16 & 0.35 & -1.67 & -0.94 & -1.10 & -0.09 & -0.17 & -0.16 & -0.08 & -0.13 & -0.04 & -0.07 & -0.01 & -0.04 & -0.01 & -0.02 & -0.01 & 2.99 & -3.00 \\
\hline$\sum_{k \in \mathcal{K}} s_{1_{k}}$ & 1 & 2 & 3 & 4 & 5 & 6 & 7 & 7 & 7 & 7 & 7 & 7 & 7 & 7 & 7 & 7 & 7 & 7 & 7 & 7 & 6 & 7 \\
\hline$\sum_{k, m \in \mathcal{T}} s_{2} s_{k m}$ & 0 & 0 & 0 & 0 & 0 & 0 & 0 & 1 & 2 & 3 & 4 & 5 & 6 & 7 & 8 & 9 & 10 & 11 & 12 & 13 & 15 & 15 \\
\hline Tempo $[\mathrm{s}]$ & 0.497 & 0.200 & 0.321 & 0.289 & 0.267 & 0.424 & 0.341 & 0.522 & 1.738 & 2.385 & 3.290 & 2.962 & 3.133 & 2.759 & 2.098 & 2.249 & 0.970 & 0.904 & 1.049 & 0.697 & 0.138 & 0.217 \\
\hline \multicolumn{23}{|c|}{ IPOPT } \\
\hline $\bar{\epsilon}$ & 1 & 2 & 3 & 4 & 5 & 6 & 7 & 8 & 9 & 10 & $11^{*}$ & 12 & 13 & 14 & $15^{*}$ & 16 & $17^{*}$ & $18^{*}$ & $19^{*}$ & 20 & 21 & 22 \\
\hline Perdas $[\mathrm{MW}]$ & - & 26.21 & 25.63 & 25.27 & 25.24 & 23.20 & 23.13 & 22.03 & 21.91 & 21.90 & 23.03 & 21.68 & 21.57 & 21.53 & 21.65 & 21.52 & 21.60 & 21.54 & 21.56 & 21.23 & 21.20 & 21.19 \\
\hline$\Delta \mathrm{P}[\mathrm{MW}]$ & - & - & -0.58 & -0.36 & -0.03 & -2.04 & -0.07 & -1.10 & -0.12 & -0.01 & 1.13 & -1.35 & -0.11 & -0.04 & 0.12 & -0.13 & 0.08 & -0.06 & 0.02 & -0.33 & -0.03 & -0.01 \\
\hline$\sum_{k \in \mathcal{B}} s_{1_{k}}$ & - & 1 & 3 & 4 & 5 & 6 & 7 & 7 & 7 & 7 & 7 & 7 & 7 & 7 & 7 & 7 & 7 & 7 & 7 & 7 & 7 & 7 \\
\hline$\sum_{k, m \in \mathcal{T}} s_{2} s_{k m}$ & - & 1 & 0 & 0 & 0 & 0 & 0 & 1 & 2 & 3 & 4 & 5 & 6 & 7 & 8 & 9 & 10 & 11 & 12 & 13 & 14 & 15 \\
\hline$s_{1}^{(0)}$ & - & 1 & 1 & 1 & 1 & 1 & 1 & 1 & 1 & 0.5 & 1 & 1 & 1 & 1 & 1 & 1 & 1 & 1 & 1 & 1 & 0 & 1 \\
\hline$s_{2}^{(0)}$ & - & 0.5 & 0.5 & 0 & 0.5 & 0.5 & 0 & 0 & 0.5 & 0.5 & 0.5 & 0 & 0 & 0 & 0 & 0 & 0.5 & 0 & 0.5 & 0.5 & 0 & 0 \\
\hline$\frac{2}{T e m p o}[\mathrm{~s}]$ & - & 0.826 & 0.353 & 0.874 & 0.635 & 0.374 & 0.262 & 0.890 & 0.626 & 0.637 & 0.617 & 0.734 & 0.950 & 0.680 & 0.416 & 0.613 & 0.625 & 0.719 & 0.882 & 0.635 & 0.974 & 0.369 \\
\hline
\end{tabular}


Tabela 6.30 - Resultados para o sistema IEEE de 118 barras, somente com soluções não dominadas.

\begin{tabular}{|c|c|c|c|c|c|c|c|c|c|c|c|c|}
\hline \multicolumn{13}{|c|}{ DICOPT } \\
\hline$\epsilon$ & 5 & 10 & 15 & 20 & 25 & 35 & 40 & 45 & 50 & 55 & 60 & 63 \\
\hline Perdas [MW] & - & 131.51 & - & 125.97 & - & - & 122.29 & 119.89 & 116.15 & 115.04 & 111.74 & 106.15 \\
\hline$\Delta \mathrm{P}[\mathrm{MW}]$ & - & - & - & -5.54 & - & - & -3.68 & -2.40 & -3.74 & -1.11 & -3.30 & -3.39 \\
\hline$\sum_{k \in \mathcal{B}} s_{1_{k}}$ & - & 9 & - & 19 & - & - & 40 & 44 & 47 & 47 & 52 & 54 \\
\hline$\sum_{k, m \in \mathcal{T}} s_{2_{k m}}$ & - & 1 & - & 1 & - & - & 0 & 1 & 3 & 8 & 8 & 9 \\
\hline Tempo $[\mathrm{s}]$ & - & 1001.171 & - & 844.107 & - & - & 146.572 & 177.796 & 118.749 & 3.561 & 2.111 & 0.524 \\
\hline \multicolumn{13}{|c|}{ KNITRO } \\
\hline$\epsilon$ & 5 & 10 & 15 & 20 & 25 & 35 & 40 & 45 & 50 & 55 & 60 & 63 \\
\hline Perdas [MW] & 133.48 & 127.25 & - & - & - & - & - & 114.39 & 110.86 & 107.34 & 106.24 & 106.15 \\
\hline$\Delta \mathrm{P}[\mathrm{MW}]$ & - & -6.23 & - & - & - & - & - & -12.86 & -3.53 & -3.52 & -1.10 & -0.09 \\
\hline$\sum_{k \in \mathcal{B}} s_{1_{k}}$ & 3 & 9 & - & - & - & - & - & 44 & 46 & 54 & 54 & 54 \\
\hline$\sum_{k, m \in \mathcal{T}} s_{2_{k m}}$ & 2 & 1 & - & - & - & 一 & - & 1 & 4 & 1 & 6 & 9 \\
\hline Tempo $[\mathrm{s}]$ & 95.812 & 305.828 & - & - & - & - & - & 463.933 & 316.275 & 60.353 & 17.780 & 0.836 \\
\hline \multicolumn{13}{|c|}{ IPOPT } \\
\hline$\epsilon$ & 5 & 10 & 15 & 20 & 25 & 35 & 40 & 45 & 50 & 55 & 60 & 63 \\
\hline Perdas [MW] & 131.62 & - & 130.28 & 130.00 & 127.11 & 125.04 & 124.07 & 120.83 & 118.27 & 107.34 & 106.24 & 106.15 \\
\hline$\Delta \mathrm{P}[\mathrm{MW}]$ & - & - & -1.34 & -0.28 & -2.89 & -2.07 & -0.97 & -3.24 & -2.56 & -10.93 & -1.10 & -0.09 \\
\hline$\sum_{k \in \mathcal{B}} s_{1_{k}}$ & 4 & - & 14 & 19 & 23 & 35 & 31 & 45 & 41 & 54 & 54 & 54 \\
\hline$\sum_{k, m \in \mathcal{T}} s_{2_{k m}}$ & 1 & - & 1 & 1 & 2 & 0 & 9 & 0 & 9 & 1 & 6 & 8 \\
\hline$s_{1}^{(0)}$ & 0 & - & 1 & 1 & 1 & 1 & 0 & 1 & 1 & 1 & 1 & 1 \\
\hline$s_{2}^{(0)}$ & 0 & - & 0.5 & 1 & 1 & 0 & 0 & 0 & 0.5 & 0.5 & 0.5 & 1 \\
\hline Tempo $[\mathrm{s}]$ & 1.163 & - & 1.352 & 2.718 & 1.925 & 0.831 & 1.298 & 1.354 & 1.529 & 0.842 & 1.011 & 1.304 \\
\hline
\end{tabular}


Tabela 6.31 - Resultados para o sistema IEEE de 300 barras, somente com soluções não dominadas.

\begin{tabular}{|c|c|c|c|c|c|c|c|c|c|c|c|c|c|c|c|c|c|}
\hline \multicolumn{18}{|c|}{ DICOPT } \\
\hline $\bar{\epsilon}$ & 10 & 20 & 30 & 40 & 50 & 60 & 70 & 80 & 90 & 100 & 110 & 120 & 140 & 150 & 160 & 170 & 176 \\
\hline Perdas $[\mathrm{MW}]$ & $\begin{array}{l}391.62 \\
\end{array}$ & 389.88 & - & - & - & - & - & - & - & - & 366.11 & 365.31 & 359.29 & 355.21 & - & 342.32 & - \\
\hline$\Delta \mathrm{P}[\mathrm{MW}]$ & - & -1.74 & - & - & - & - & - & - & - & - & -23.77 & -0.80 & -6.02 & -4.08 & - & -12.89 & - \\
\hline$\sum_{k \in \mathcal{B}} s_{1_{k}}$ & 3 & 11 & - & - & - & - & - & - & - & - & 40 & 42 & 49 & 58 & - & 64 & - \\
\hline$\sum_{k, m \in \mathcal{T}} s_{2}{ }_{k m}$ & 7 & 9 & - & - & - & - & - & - & - & - & 70 & 78 & 91 & 92 & - & 106 & - \\
\hline Tempo $[\mathrm{s}]$ & 219.998 & 1000.550 & - & - & - & - & - & - & - & - & 1000.675 & 1000.702 & 1000.698 & 1000.527 & - & 2.997 & - \\
\hline \multicolumn{18}{|c|}{ KNITRO } \\
\hline $\bar{\epsilon}$ & 10 & 20 & 30 & 40 & 50 & 60 & 70 & 80 & 90 & 100 & 110 & 120 & 140 & 150 & 160 & 170 & 176 \\
\hline Perdas $[\mathrm{MW}]$ & - & - & - & - & - & - & - & - & - & - & - & - & - & - & 342.99 & 342.47 & 342.32 \\
\hline$\Delta \mathrm{P}[\mathrm{MW}]$ & - & - & - & - & - & - & - & - & - & - & - & - & - & - & - & -0.52 & -0.15 \\
\hline$\sum_{k, m \in \mathcal{T}} s_{2}{ }_{k m}$ & - & - & - & - & - & - & - & - & - & - & - & - & - & - & 102 & 106 & 107 \\
\hline Tempo [s] & - & - & - & - & - & - & - & - & - & - & - & - & - & - & 583.622 & 15.664 & 0.707 \\
\hline \multicolumn{18}{|c|}{ IPOPT } \\
\hline $\bar{\epsilon}$ & 10 & 20 & 30 & 40 & 50 & 60 & 70 & 80 & 90 & 100 & 110 & 120 & 140 & 150 & 160 & 170 & 176 \\
\hline Perdas $[\mathrm{MW}]$ & - & 402.05 & 394.45 & 392.41 & 379.83 & 371.02 & 356.80 & 351.74 & 348.80 & 348.36 & 347.08 & 345.65 & - & 345.23 & 343.98 & 342.39 & 342.32 \\
\hline$\Delta \mathrm{P}[\mathrm{MW}]$ & - & - & -7.60 & -2.04 & -12.58 & -8.81 & -14.22 & -5.06 & -2.94 & -0.44 & -1.28 & -1.43 & - & -0.42 & -1.25 & -1.59 & -0.07 \\
\hline Perdas $[\mathrm{MW}]$ & - & 4 & 30 & 40 & 50 & 60 & 69 & 69 & 69 & 69 & 69 & 68 & - & 69 & 69 & 69 & 69 \\
\hline$\Delta \mathrm{P}[\mathrm{MW}]$ & - & 16 & 0 & 0 & 0 & 0 & 1 & 11 & 21 & 31 & 41 & 52 & - & 81 & 91 & 101 & 107 \\
\hline Perdas $[\mathrm{MW}]$ & - & 0 & 1 & 1 & 1 & 1 & 1 & 1 & 1 & 1 & 1 & 1 & - & 1 & 1 & 1 & 1 \\
\hline$\Delta \mathrm{P}[\mathrm{MW}]$ & - & 0.5 & 0 & 0.5 & 0.5 & 0.5 & 0.5 & 0 & 0.5 & 0.5 & 0.5 & 1 & - & 0.5 & 0 & 0 & 0 \\
\hline$\epsilon$ & - & 3.852 & 5.630 & 2.676 & 2.851 & 3.159 & 5.647 & 3.047 & 2.615 & 2.827 & 2.370 & 3.189 & - & 3.530 & 5.054 & 5.317 & 3.146 \\
\hline
\end{tabular}




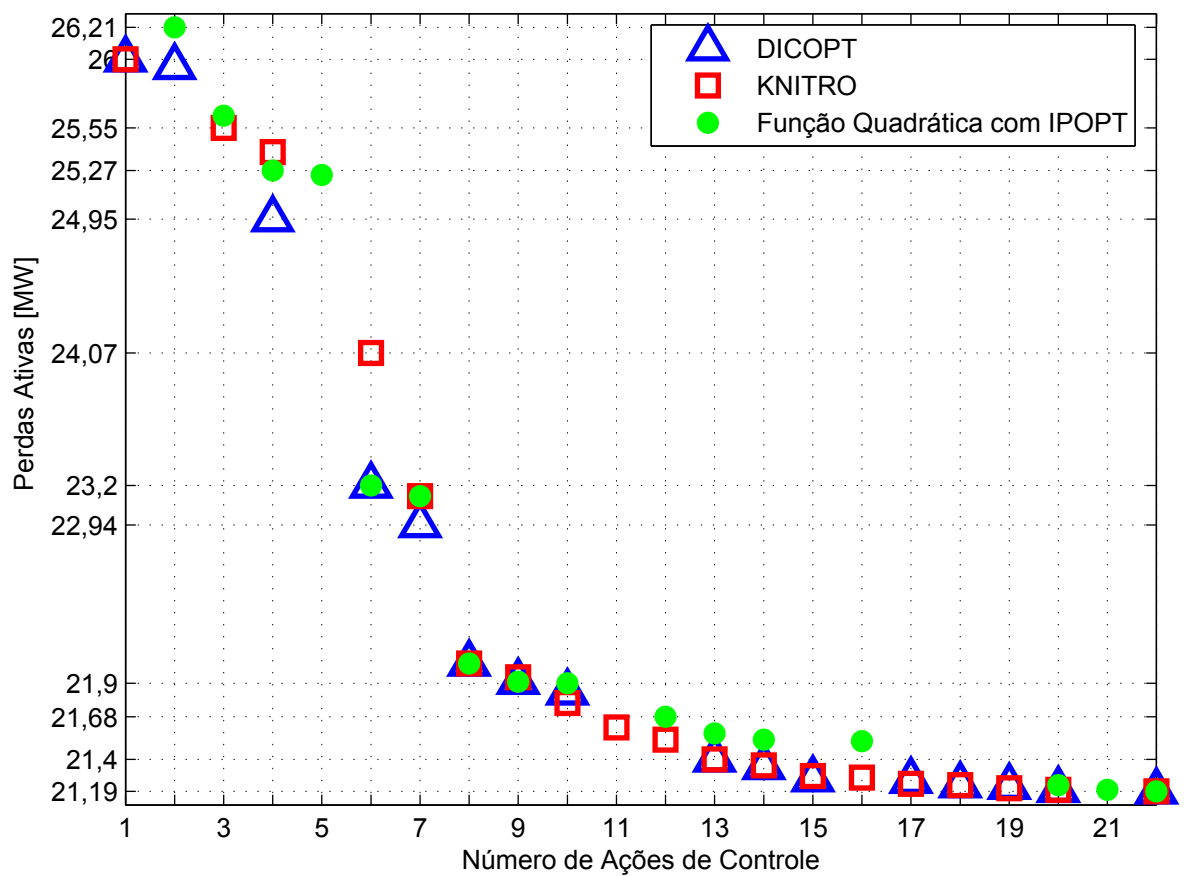

Figura 6.27 - Perdas ativas pelo número de ações de controle para o sistema de 57 barras com todos os solvers (Método $\epsilon$-Restrito).

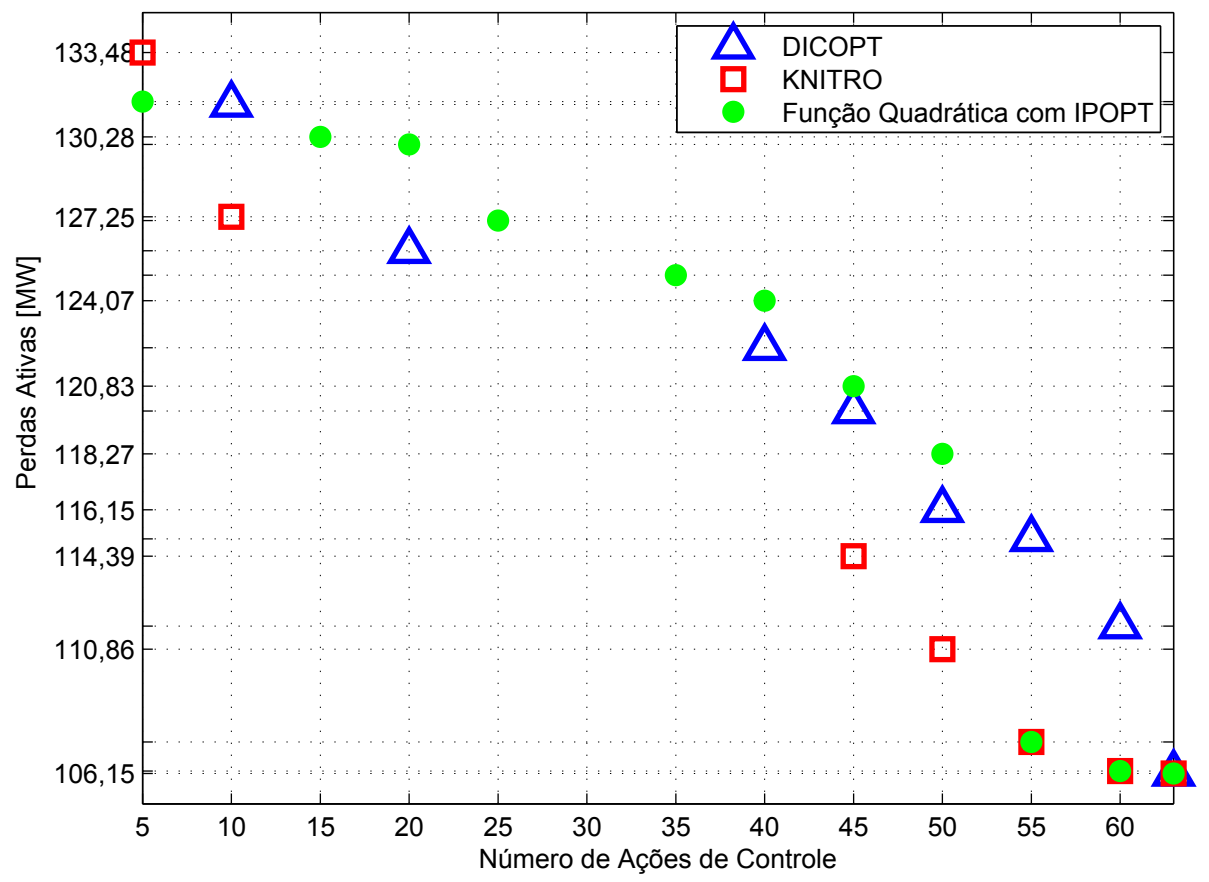

Figura 6.28 - Perdas ativas pelo número de ações de controle para o sistema de 118 barras com todos os solvers (Método $\epsilon$-Restrito). 


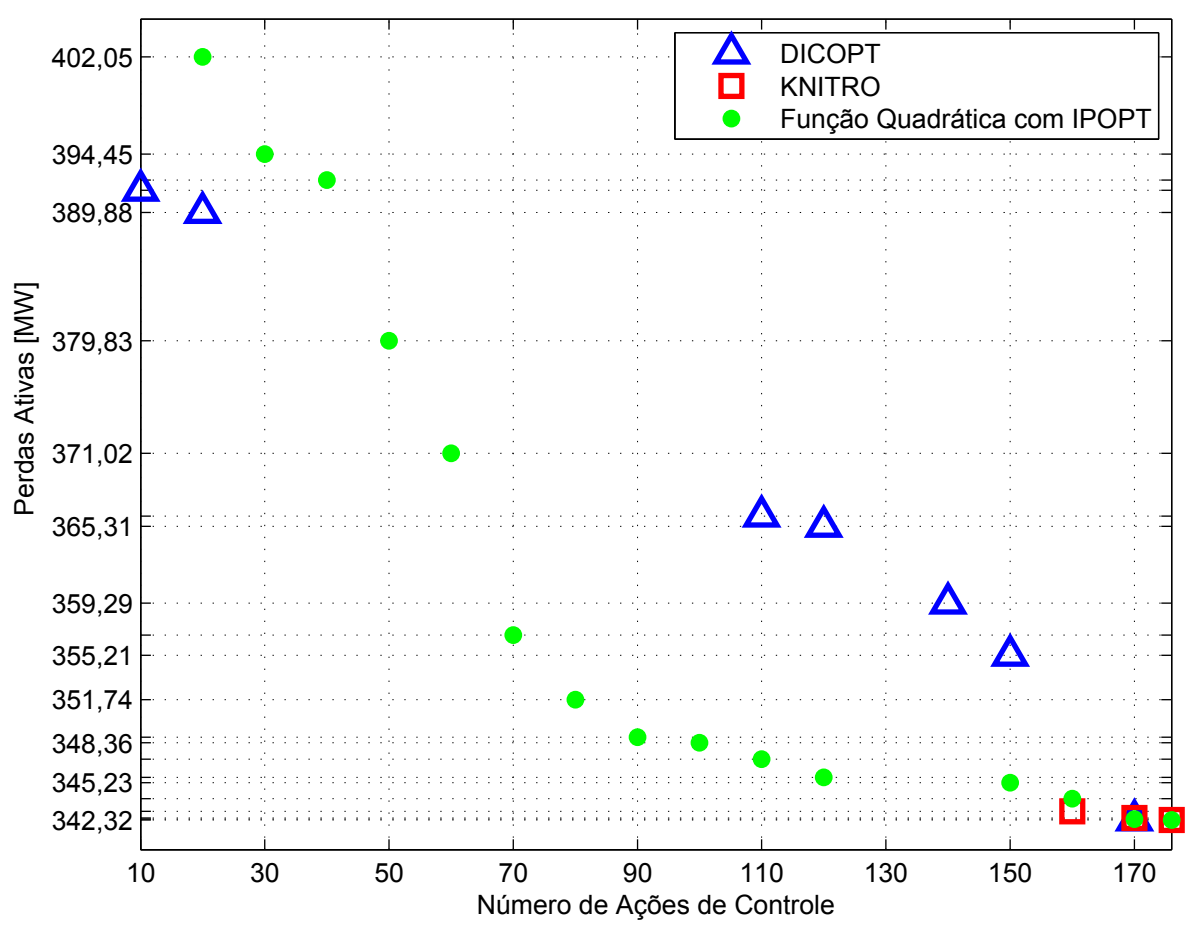

Figura 6.29 - Perdas ativas pelo número de ações de controle para o sistema de 300 barras com todos os solvers (Método $\epsilon$-Restrito).

\section{- Análise dos Resultados Obtidos}

Foram comparadas as soluções encontradas pelos solver DICOPT e KNITRO para o problema (5.5) com as soluções obtidas pelo solver IPOPT para o problema modificado (5.6). Comparando ponto-a-ponto e eliminando as soluções dominadas entre os solvers DICOPT e KNITRO para o modelo (5.5) e o solver IPOPT para o modelo (5.6) obtemos a curva combinada de cada sistema.

Para o sistema de 14 barras o solver DICOPT possui sete soluções na curva combinada, KNITRO possui cinco enquanto IPOPT possui três soluções na curva combinada. A Figura 6.30 apresenta a curva combinada das soluções não dominadas para os três solvers.

Para o sistema de 30 barras, DICOPT domina KNITRO em uma solução e as demais soluções são equivalentes. Comparando DICOPT com o IPOPT, temos que DICOPT domina IPOPT em oito soluções, e as demais são similares. Para traçar a curva combinada, basta ignorarmos as soluções de KNITRO e IPOPT. Para o sistema de 30 barras, DICOPT domina KNITRO em uma solução e as demais soluções são equivalentes. Comparando com o IPOPT, DICOPT domina IPOPT em oito soluções, e as demais são similares. Para traçar a curva combinada, basta ignorarmos as soluções de KNITRO e IPOPT. Já para o sistema de 57 barras, DICOPT têm desempenho igual ao KNITRO em seis soluções, domina KNITRO em seis soluções e é dominado por KNITRO em seis soluções. Ao comparar DICOPT e IPOPT ambos possuem quatro soluções de desempenho igual, sendo que duas delas também coincidem com KNITRO. IPOPT é dominado por KNITRO e/ou DICOPT em catorze soluções. Ou seja, DICOPT tem 


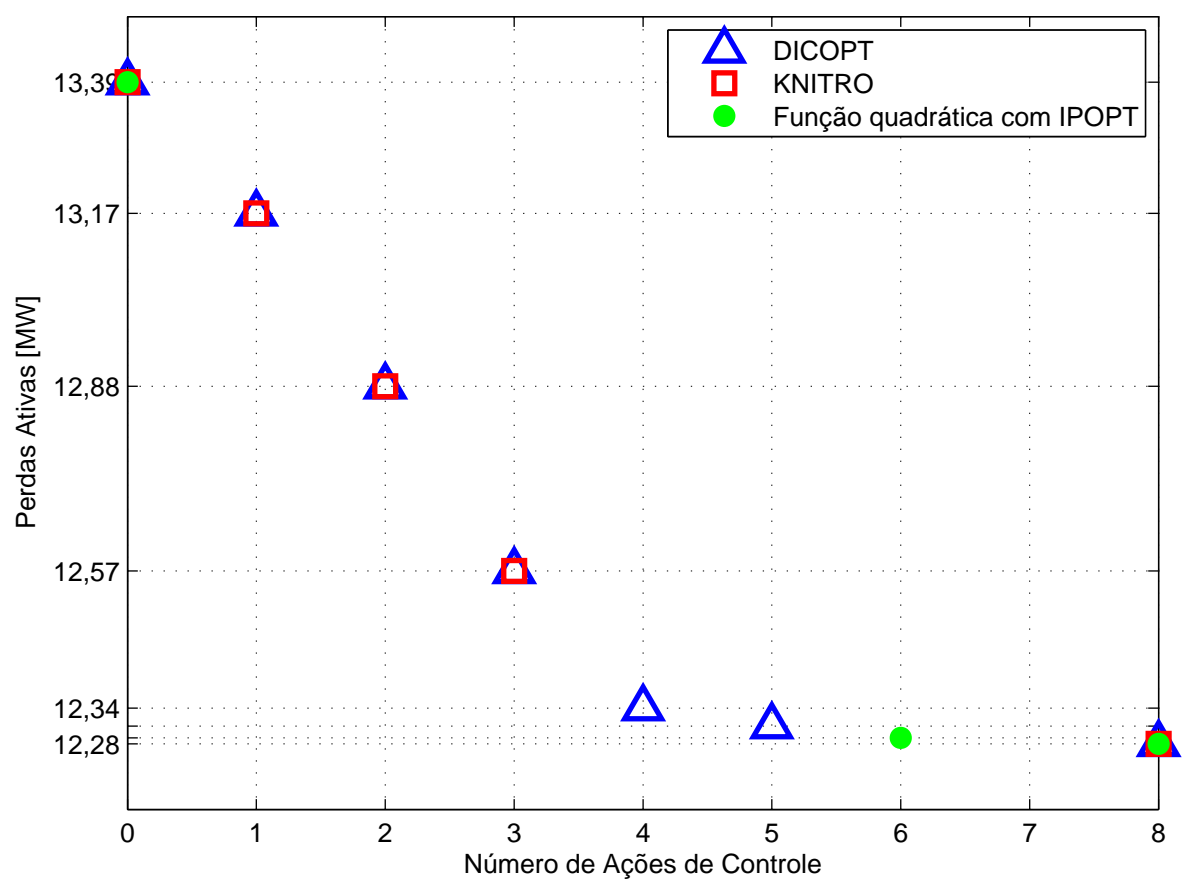

Figura 6.30 - Perdas ativas pelo número de ações de controle da curva combinada do sistema de 14 barras. Soluções não dominadas geradas pela combinação das soluções dos três solvers (Método $\epsilon$-Restrito).

doze soluções na curva combinada, KNITRO também tem doze soluções e IPOPT tem apenas 4 soluções na curva combinada. Este efeito pode ser visto na Figura 6.31.

Para o sistema IEEE de 118 barras, foram testados doze valores para $\epsilon$, DICOPT encontrou oito soluções factíveis, KNITRO encontrou sete soluções e IPOPT encontrou onze. Comparando ponto-a-ponto entre os solvers e removendo soluções dominadas geramos a curva combinada representada na Figura 6.32. Observe que DICOPT possui três soluções na curva combinada, KNITRO possui seis e IPOPT possui cinco soluções.

O mesmo efeito que ocorre com o método da soma ponderada ocorre com o método $\epsilon$-Restrito, a medida que a quantidade de variáveis binárias aumentam, a eficiência dos métodos de PNLIM diminui.

Para o sistema de 300 barras, foram utilizados dezessete valores para $\epsilon$. DICOPT não encontra a melhor solução possível, parando o processo por deterioramento do problema, encontrando apenas sete soluções factíveis para o problema. KNITRO por sua vez, encontra apenas soluções para três dos dezessete valores testados. IPOPT encontra soluções para 15 dos 17 valores de $\epsilon$. Comparando ponto-a-ponto e removendo soluções dominadas entre os solvers KNITRO e CONOPT obtemos a Figura 6.33. 


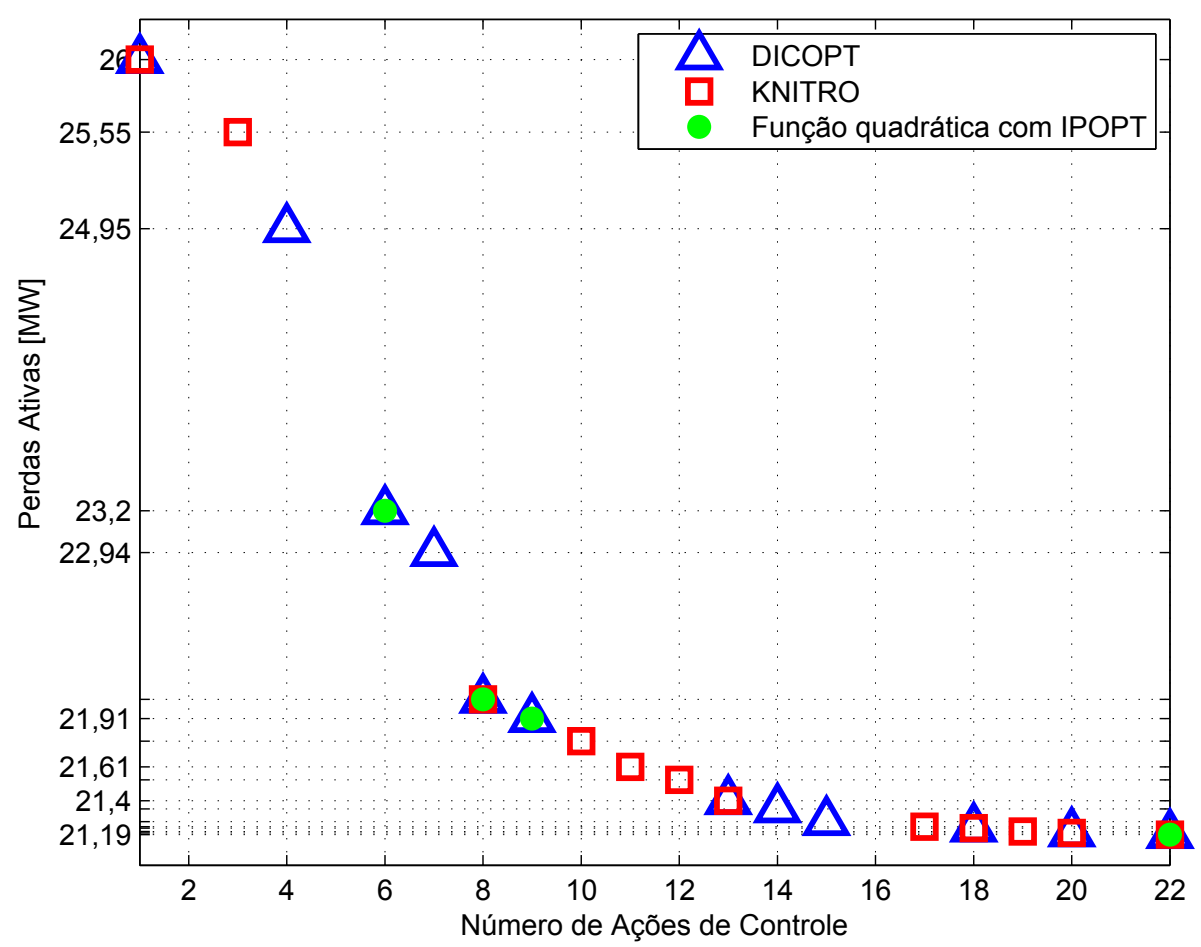

Figura 6.31 - Perdas ativas pelo número de ações de controle da curva combinada do sistema de 57 barras. Soluções não dominadas geradas pela combinação das soluções dos três solvers (Método $\epsilon$-Restrito).

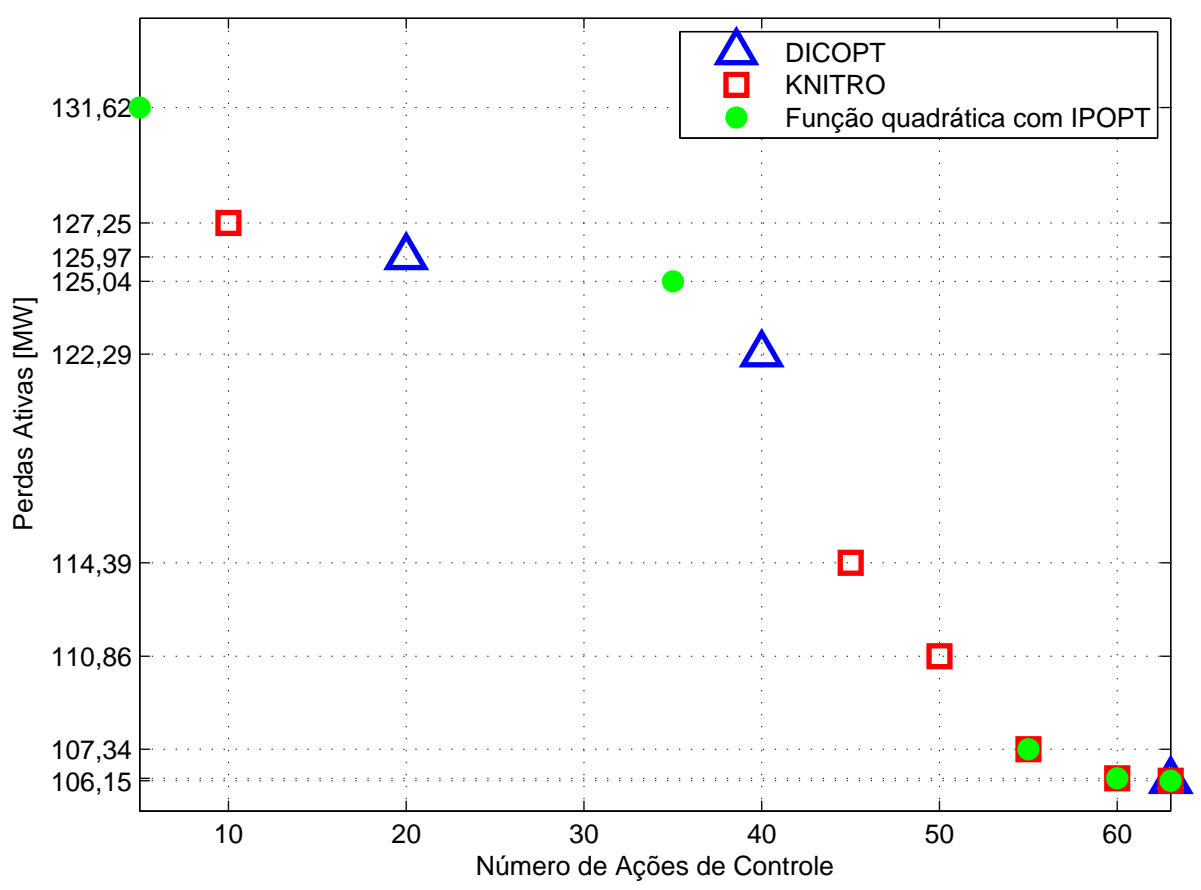

Figura 6.32 - Perdas ativas pelo número de ações de controle da curva combinada do sistema de 118 barras. Soluções não dominadas geradas pela combinação das soluções dos três solvers (Método $\epsilon$-Restrito). 


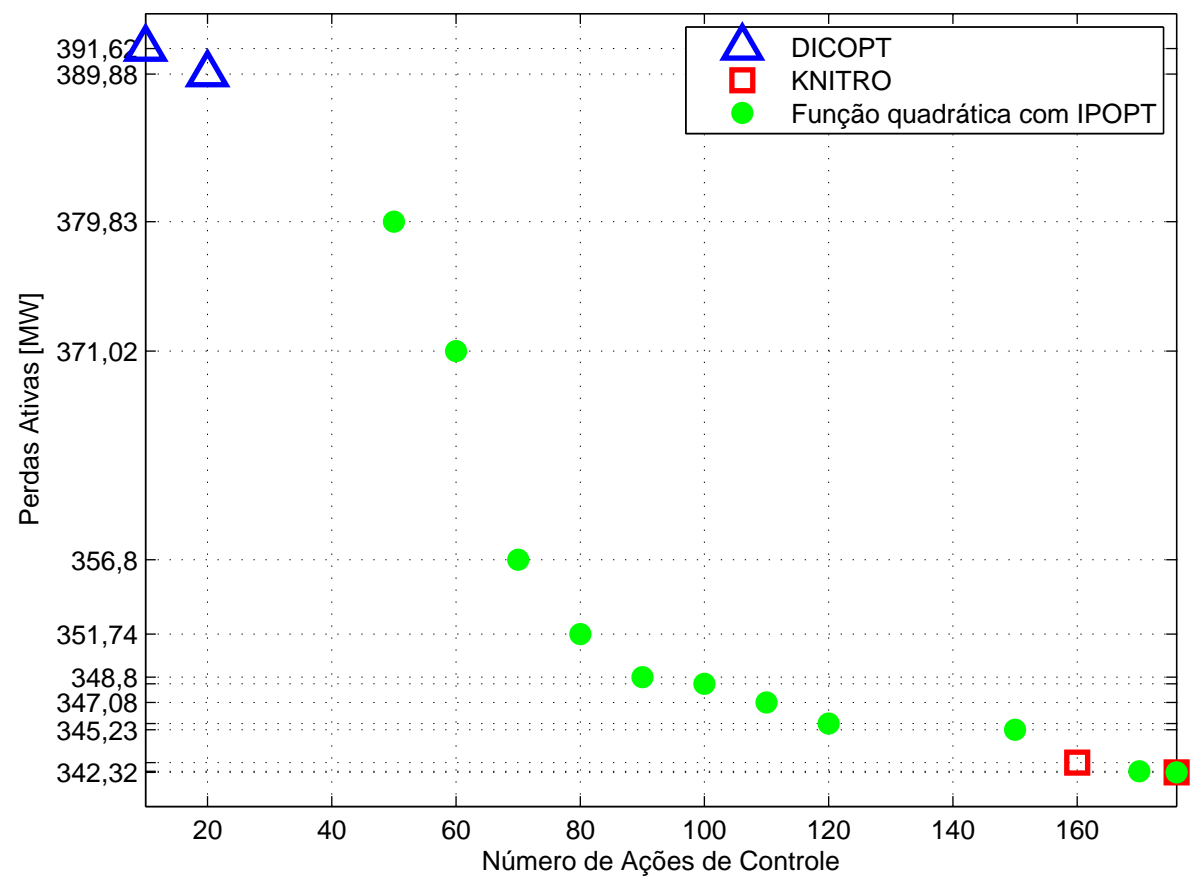

Figura 6.33 - Perdas ativas pelo número de ações de controle da curva combinada do sistema de 300 barras. Soluções não dominadas geradas pela combinação das soluções dos três solvers (Método $\epsilon$-Restrito).

\subsubsection{Comparação entre Soma Ponderada e $\epsilon$-Restrito}

Deixamos de lado aqui os resultados encontrados por DICOPT e KNITRO para os problemas (5.2) e (5.5), que são equivalentes ao problema de FPO Multiobjetivo com taps de transformadores contínuos e bancos de capacitores e reatores shunts fixos modificado pelos métodos da soma ponderada e $\epsilon$-restrito, respectivamente.

Consideramos o modelo (5.3) resolvido pelo Algoritmo 5 com o solver CONOPT, lembrando que o mesmo usa a função sigmoidal para o tratamento das variáveis binárias, esta estratégia será chamada somente de Soma Ponderada. Consideramos também o modelo (5.6), que utiliza a função quadrática na modelagem das variáveis binárias, resolvido pelo solver IPOPT, nomeamos esta estratégia somente de $\epsilon$-Restrito.

Antes de iniciar esta comparação é importante devemos deixar claro que o solver IPOPT foi testado junto com o Algoritmo 5 para a resolução do problema (5.3), mas se mostrou muito inferior ao CONOPT, e o CONOPT por sua vez foi testado para a resolução do problema (5.6), mas se comportou de forma instável. Por conta destes fatos, tais resultados não foram apresentados neste capítulo.

Para o sistema de 14 barras, o total de soluções não dominadas individualmente foram cinco para a Soma Ponderada, sendo que todas elas estão na curva combinada, e o $\epsilon$-Restrito encontrou oito soluções não dominadas individualmente, das quais apenas 4 estão na curva combinada. A Figura 6.34 apresenta a curva combinada das soluções não dominadas para a Soma Ponderada e o $\epsilon-$ Restrito. 


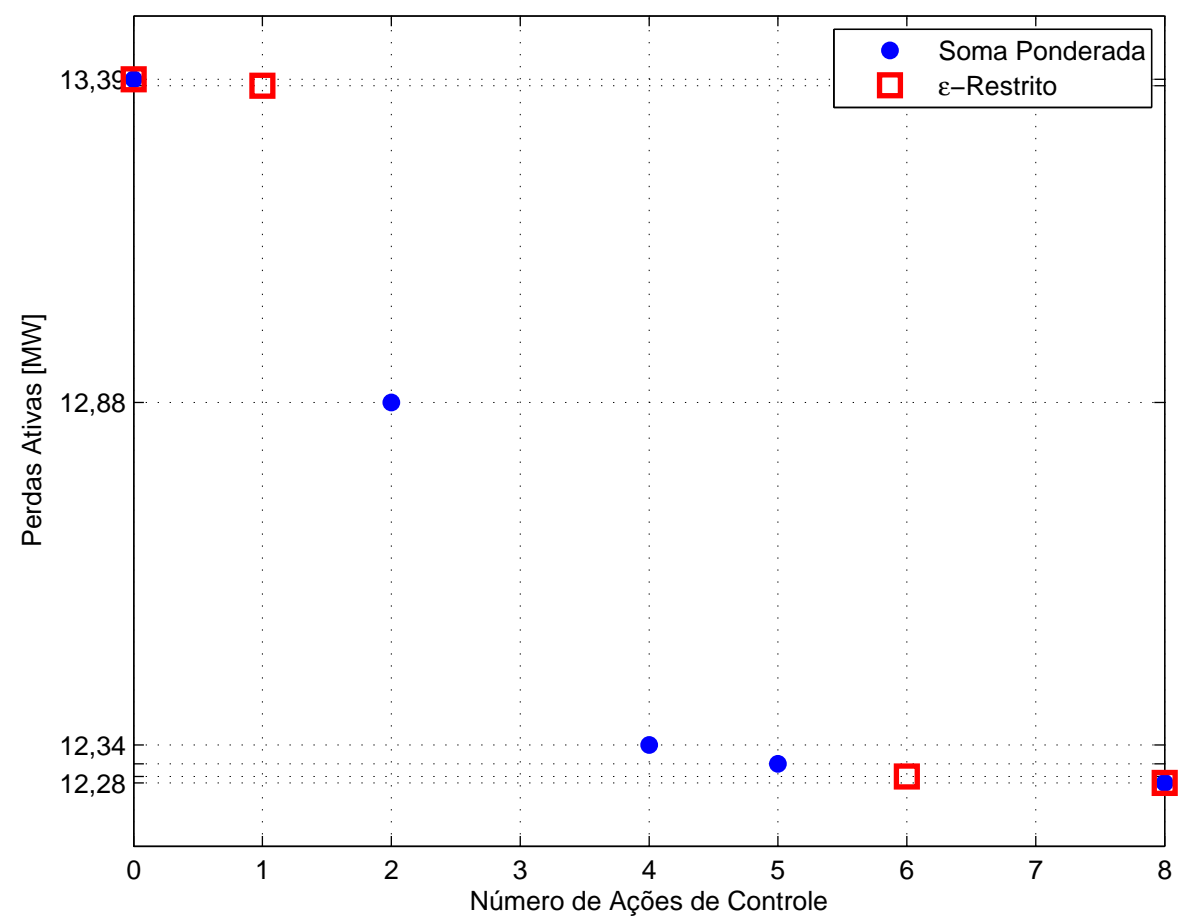

Figura 6.34 - Perdas ativas pelo número de ações de controle da curva combinada do sistema de 14 barras entre a Soma Ponderada e o $\epsilon$-Restrito.

Para o sistema de 30 barras, um total de sete soluções não dominadas individualmente foram obtidas com a Soma Ponderada, sendo que todas elas estão na curva combinada, e o $\epsilon$-Restrito encontrou dez soluções não dominadas individualmente, onde apenas quatro estão na curva combinada. A Figura 6.35 apresenta a curva combinada das soluções não dominadas para a Soma Ponderada e o $\epsilon$-Restrito.

Para o sistema de 57 barras, o total de soluções não dominadas individualmente foram sete para a Soma Ponderada, sendo que seis estão na curva combinada, e $\epsilon$-Restrito encontrou dezesseis soluções não dominadas individualmente, onde doze estão na curva combinada. A Figura 6.36 apresenta a curva combinada das soluções não dominadas para a Soma Ponderada e o $\epsilon$-Restrito.

Para o sistema de 118 barras, o total de soluções não dominadas individualmente foram treze para a Soma Ponderada, sendo que todas estão na curva combinada, e $\epsilon$-Restrito encontrou onze soluções não dominadas individualmente, onde apenas duas estão na curva combinada. A Figura 6.37 apresenta a curva combinada das soluções não dominadas para a Soma Ponderada e o $\epsilon$-Restrito.

Para o sistema de 300 barras, o total de soluções não dominadas individualmente foram onze para a Soma Ponderada, sendo que todas estão na curva combinada, e $\epsilon$-Restrito encontrou quinze soluções não dominadas individualmente, onde apenas duas estão na curva combinada. A Figura 6.38 apresenta a curva combinada das soluções não dominadas para a Soma Ponderada e o $\epsilon$-Restrito. 


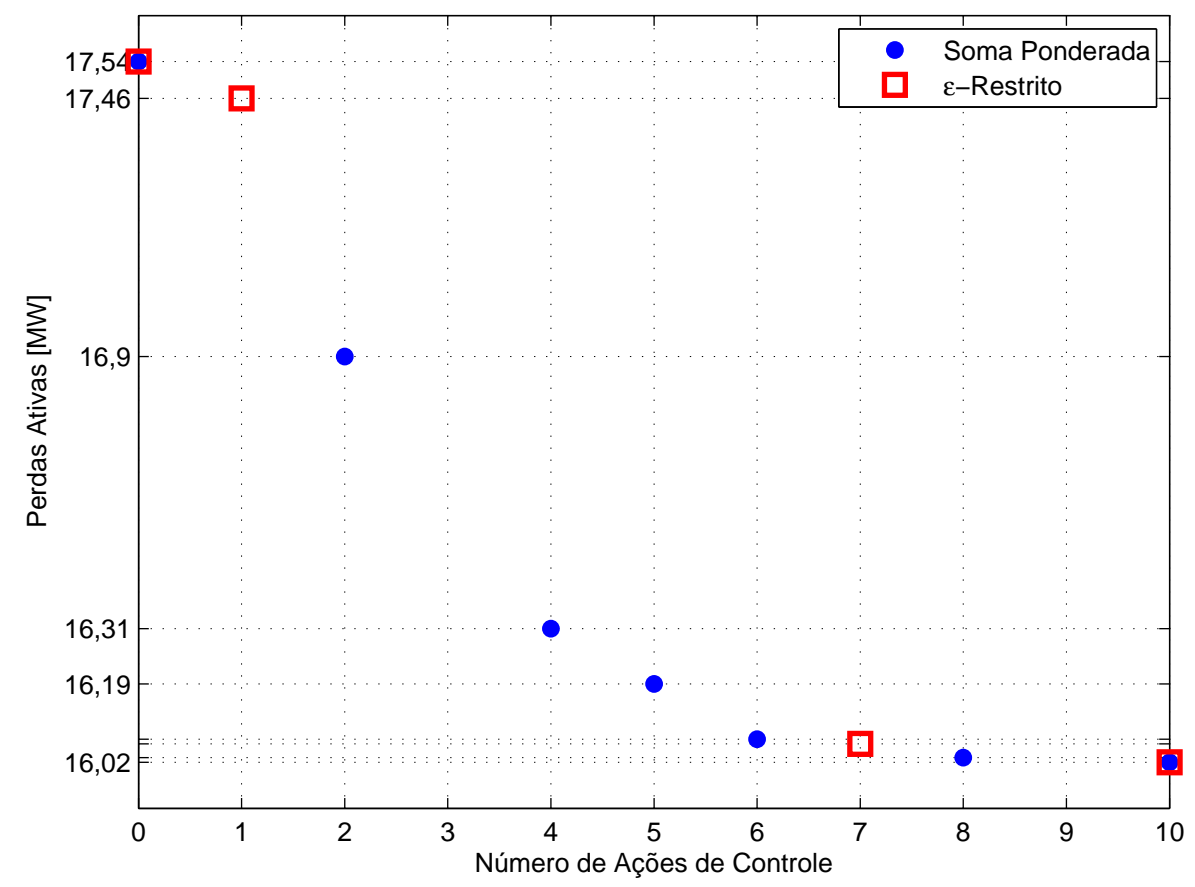

Figura 6.35 - Perdas ativas pelo número de ações de controle da curva combinada do sistema de 30 barras entre a Soma Ponderada e o $\epsilon$-Restrito.

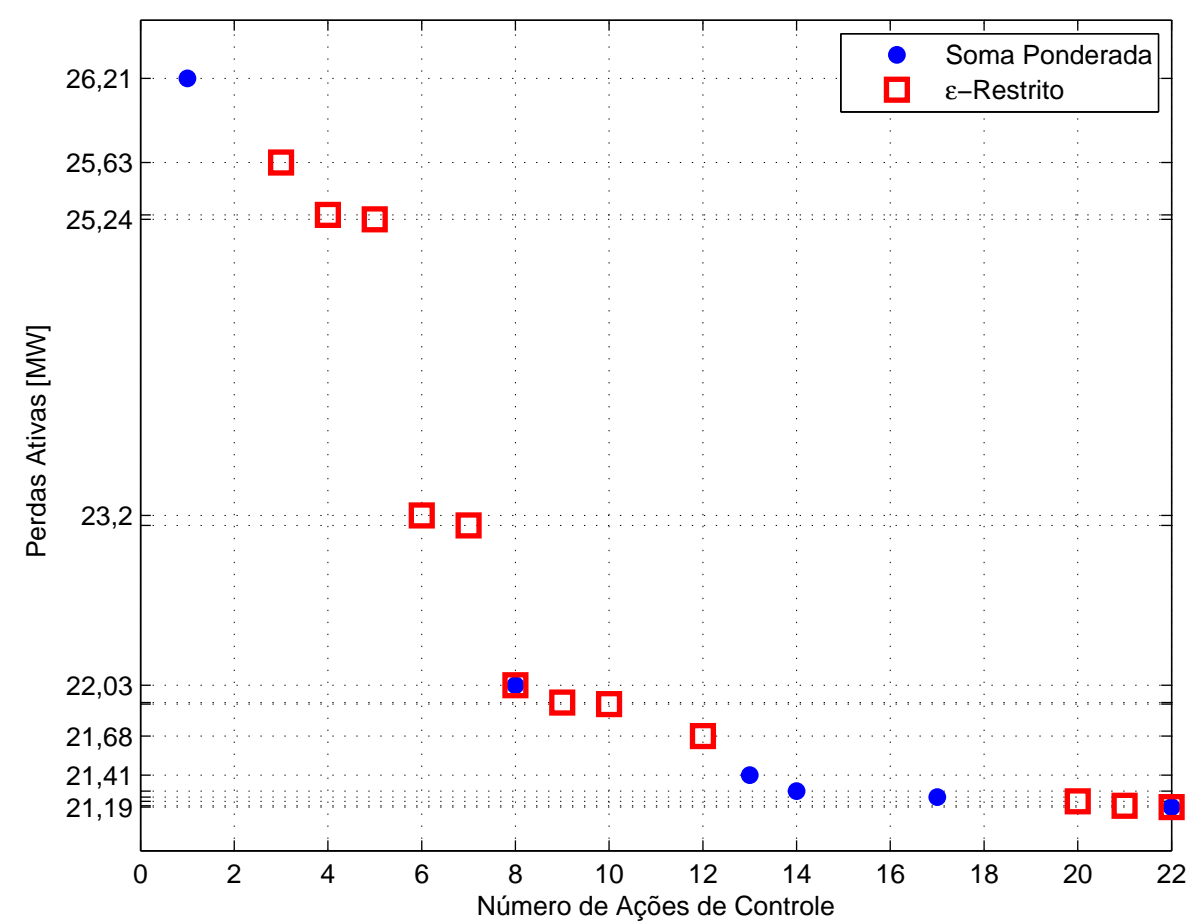

Figura 6.36 - Perdas ativas pelo número de ações de controle da curva combinada do sistema de 57 barras entre a Soma Ponderada e o $\epsilon$-Restrito. 


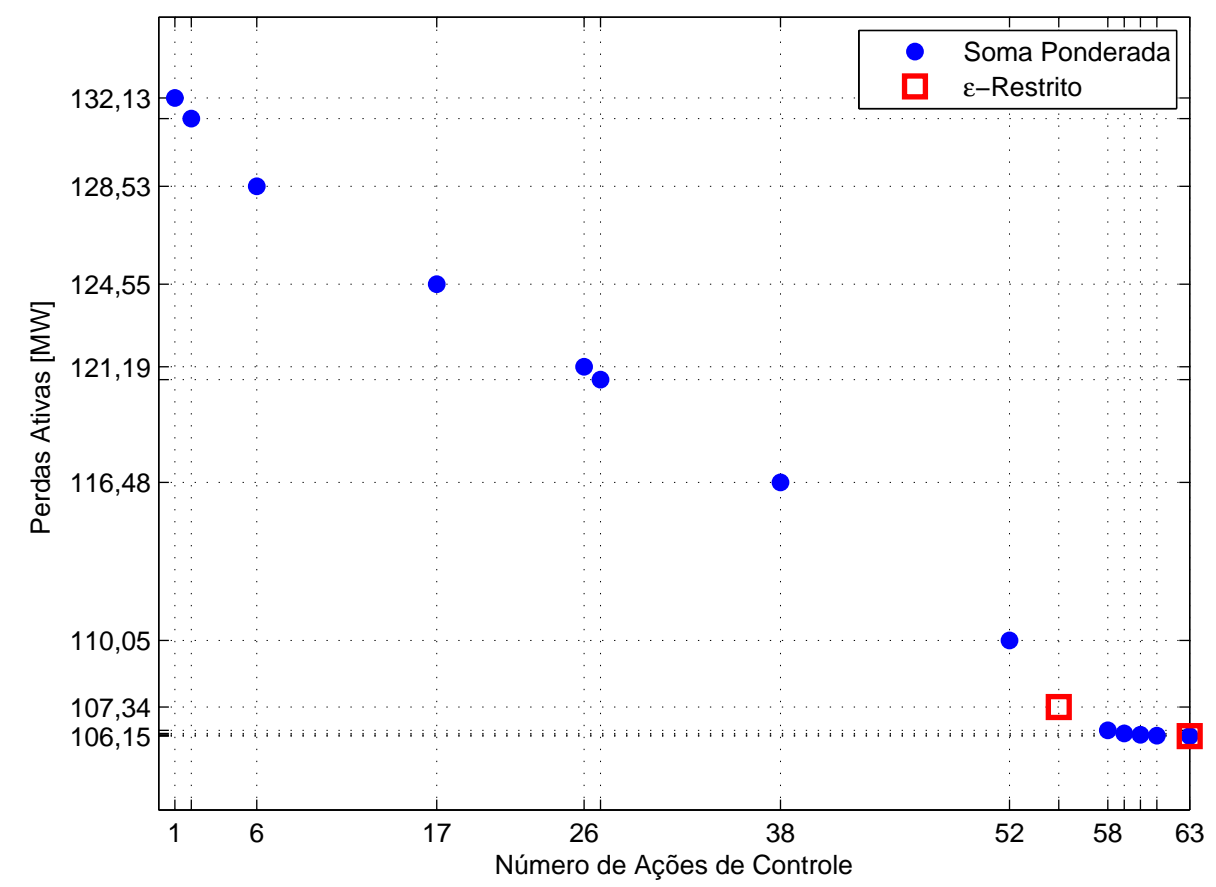

Figura 6.37 - Perdas ativas pelo número de ações de controle da curva combinada do sistema de 118 barras entre a Soma Ponderada e o $\epsilon$-Restrito.

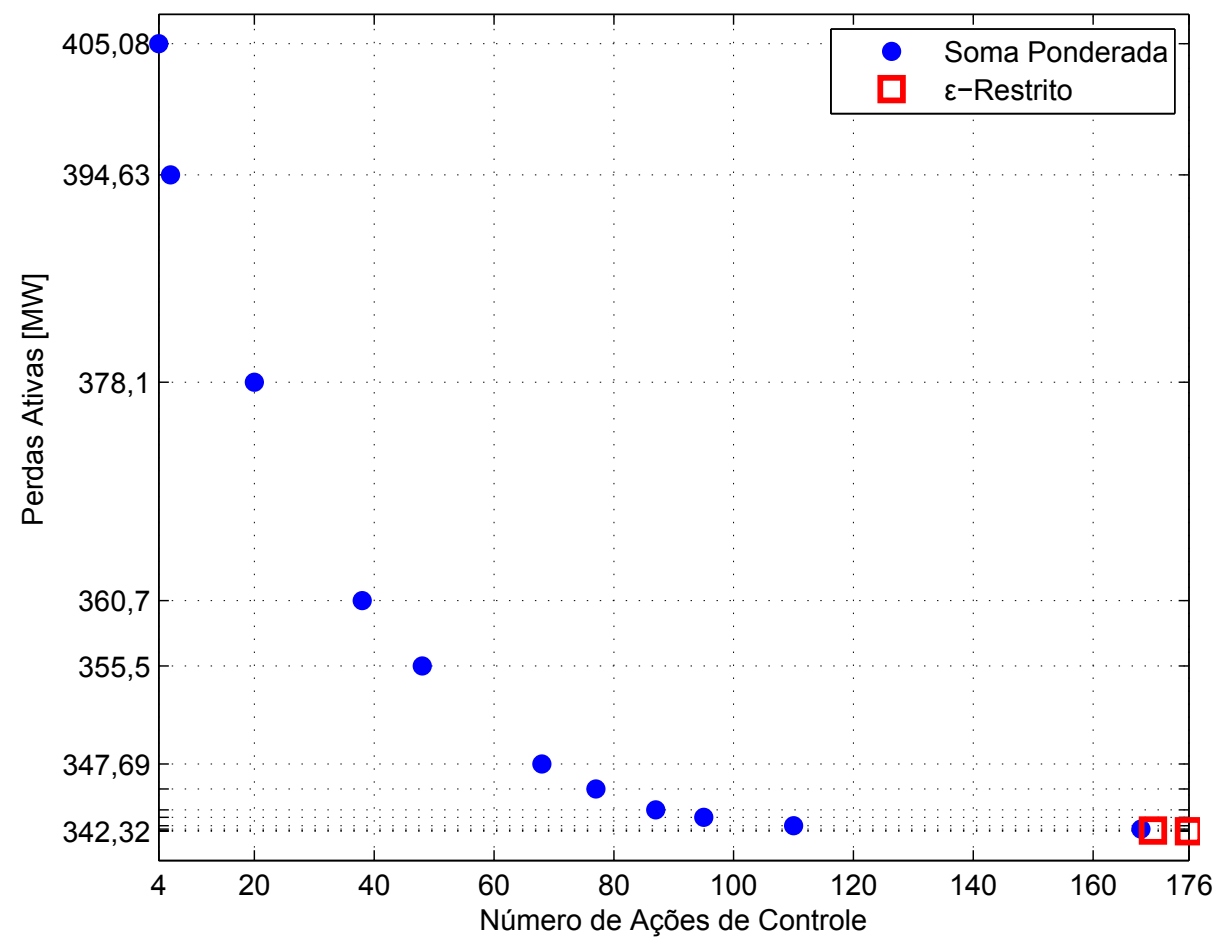

Figura 6.38 - Perdas ativas pelo número de ações de controle da curva combinada do sistema de 300 barras entre a Soma Ponderada e o $\epsilon$-Restrito. 
Podemos dizer que de modo geral, o método da Soma Ponderada com a função sigmoidal obteve melhor desempenho do que o método $\epsilon$-Restrito com a função quadrática. Comparando as soluções encontradas pelas duas estratégias para os sistemas elétricos do IEEE, o método da soma ponderada domina mais soluções que o método $\epsilon$-Restrito, perdendo apenas para o sistema de 57 barras onde o método $\epsilon$-Restrito obteve um maior número de soluções na curva combinada.

\subsubsection{Conclusões}

Nesta seção apresentamos os resultados encontrados para o modelo de FPO Multiobjetivo com taps de transformadores contínuos e os bancos de capacitores e reatores shunts fixos, onde realizou-se a verificação da eficiência das estratégias adotadas para o tratamento do modelo multiobjetivo, e também, das estratégias desenvolvidas para o tratamento das variáveis binárias associadas aos controles do problema. Os métodos da Soma Ponderada e $\epsilon$-Restrito foram utilizados para o tratamento do problema multiobjetivo, ambos os métodos modificam o problema multiobjetivo em um problema mono-objetivo. Combinado com o método da soma ponderada utilizou-se uma função sigmoidal para o tratamento das variáveis binárias e este modelo foi resolvido pelo solver CONOPT. Já para o método $\epsilon$-restrito foi utilizada uma função quadrática para o tratamento das variáveis binárias e este novo modelo foi resolvido pelo solver IPOPT. Testes foram conduzidos com os solvers DICOPT e KNITRO para verificar a eficiência da modelagem binária. Ambas as estratégias resultam em resultados factíveis para o problema multiobjetivo chegando a um compromisso entre as perdas ativas na transmissão e o número de ações de controles executados. As funções para a modelagem das variáveis binárias são eficientes e competitivas ao comparadas com os solvers DICOPT e KNITRO. Fica nítido que o algoritmo proposto utilizando a função sigmoidal em conjunto com o método da soma ponderada e CONOPT é superior aos testes feitos com DICOPT e KNITRO. Comparando os resultados com ambas as estratégias, pode-se concluir que a estratégia utilizando a soma ponderada é superior a estratégia comparando o $\epsilon$-restrito.

A próxima subseção contém os resultados para o problema de FPO multiobjetivo com taps de transformadores discretos e banco de capacitores e reatores shunts contínuos é modelado pelo método $\epsilon$-Restrito. As variáveis discretas são modeladas pela função quadrática e os taps de transformadores são modelados pela função de discretização polinomial. 


\subsubsection{FPO Multiobjetivo com Tap de Transformadores Discretos}

Considere o modelo (5.7) para o problema de FPO multiobjetivo com tap de transformadores discretos e elementos shunts fixos. O modelo (5.7) será tratado pelo método $\epsilon$-restrito tornando-se um problema mono-objetivo como em (5.8) no qual podemos aplicar técnicas de PNLIM. As variáveis binárias do problema (5.8) são tratadas pela função quadrática, definida na Seção 3.5, essa modificação é apresentada no problema (5.9). As variáveis discretas do problema (5.9), que são equivalente aos taps de transformadores, são tratadas pelas funções de discretização Senoidal (Algoritmo 1) e Polinomial (Algoritmo 2) apresentadas nas Seções 3.1 e 3.2 , respectivamente.

Utiliza-se o solver IPOPT na realização destes testes. As variáveis taps de transformadores são inicializadas discretas já que um dos objetivos do problema é minimizar o número de ações de controles.

A Tabela 6.32 mostra as características de cada sistema para o modelo (5.7).

Tabela 6.32 - Características dos Sistemas Elétricos

\begin{tabular}{c|ccccc}
\hline $\begin{array}{c}\text { Sistema } \\
\text { Elétrico }\end{array}$ & $\begin{array}{c}\text { Restrições de } \\
\text { Igualdade }\end{array}$ & $\begin{array}{c}\text { Variáveis } \\
\text { Contínuas }\end{array}$ & $\begin{array}{c}\text { Variáveis } \\
\text { Discretas }\end{array}$ & $\begin{array}{c}\text { Variáveis } \\
\text { de Controle }\end{array}$ & $\begin{array}{c}\text { Variáveis } \\
\text { Binárias }\end{array}$ \\
\hline 14 & 22 & 27 & 3 & 8 & 8 \\
30 & 53 & 59 & 4 & 10 & 10 \\
57 & 106 & 113 & 15 & 22 & 22 \\
118 & 181 & 235 & 9 & 63 & 63 \\
300 & 530 & 599 & 107 & 176 & 176 \\
\hline
\end{tabular}

O número de iterações apresentado nas tabelas com os resultados desta seção representa um contador interno dos algoritmos de discretização propostos, não estando relacionados com o número de iterações do solver escolhido.

Os testes foram realizados com diferentes valores de $\epsilon$ para cada sistema. Lembrando que a solução viável que apresenta um trade-off entre os objetivos não é considerada uma solução ótima de Pareto. Os parâmetros $s_{1}$ e $s_{2}$ foram inicializados de acordo com a Tabela 6.26. Os resultados apresentados são os melhores encontrados comparando os sete casos de entrada dos parâmetros $s_{1}$ e $s_{2}$.

As Tabelas $6.33,6.34,6.35,6.36$ e 6.37 apresentam os resultados obtidos para os sistemas IEEE de 14, 30, 57, 118 e 300 barras, respectivamente, com as funções Senoidal e Polinomial. O método $\epsilon$-Restrito não garante que para todos os $\epsilon$ escolhidos sejam obtidos soluções factíveis para o problema. Por exemplo, para o sistema IEEE de 30 barras, $\epsilon=0$ é infactível para todos os valores iniciais testados com $s_{1}$ e $s_{2}$. O mesmo ocorre para $\epsilon=5$ no sistema IEEE de 300 barras. 
Tabela 6.33 - Resultados para o sistema IEEE de 14 barras. Soluções com * são dominadas pelas demais.

\begin{tabular}{|c|c|c|c|c|c|c|c|c|c|}
\hline \multicolumn{10}{|c|}{ Função Senoidal } \\
\hline$\epsilon$ & 0 & 1 & $2^{*}$ & 3 & 4 & 5 & 6 & 7 & 8 \\
\hline Perdas [MW] & 13.39 & 13.17 & 13.33 & 13.14 & 12.77 & 12.40 & 12.30 & 12.29 & 12.28 \\
\hline$\Delta \mathrm{P}[\mathrm{MW}]$ & - & -0.22 & 0.16 & -0.19 & -0.37 & -0.37 & -0.10 & -0.01 & -0.01 \\
\hline$\sum_{k \in \mathcal{B}} s_{1_{k}}$ & 0 & 1 & 1 & 3 & 3 & 5 & 5 & 4 & 5 \\
\hline$\sum_{k, m \in \mathcal{T}} s_{2_{k m}}$ & 0 & 0 & 1 & 0 & 1 & 0 & 1 & 3 & 3 \\
\hline$s_{1}^{(0)}$ & 1 & 0 & 0 & 1 & 1 & 1 & 1 & 1 & 1 \\
\hline$s_{2}^{(0)}$ & 0 & 0 & 0 & 0 & 1 & 0 & 0 & 0.5 & 0 \\
\hline Tempo $[\mathrm{s}]$ & 0.569 & 0.386 & 0.368 & 0.393 & 0.425 & 0.331 & 0.394 & 0.415 & 0.483 \\
\hline Iterações & 2 & 2 & 2 & 2 & 2 & 2 & 2 & 2 & 2 \\
\hline \multicolumn{10}{|c|}{ Função Polinomial } \\
\hline $\bar{\epsilon}$ & 0 & 1 & $2^{*}$ & 3 & 4 & 5 & $6^{*}$ & 7 & 8 \\
\hline Perdas [MW] & 13.39 & 13.17 & 13.33 & 13.14 & 12.80 & 12.40 & 12.40 & 12.29 & 12.28 \\
\hline$\Delta \mathrm{P}[\mathrm{MW}]$ & - & -0.22 & 0.16 & -0.19 & -0.34 & -0.40 & 0.00 & -0.11 & -0.01 \\
\hline$\sum_{k \in \mathcal{B}} s_{1_{k}}$ & 0 & 1 & 1 & 3 & 4 & 5 & 5 & 4 & 5 \\
\hline$\sum_{k, m \in \mathcal{T}} s_{2_{k m}}$ & 0 & 0 & 1 & 0 & 0 & 0 & 1 & 3 & 3 \\
\hline$s_{1}^{(0)}$ & 0 & 0 & 0 & 1 & 1 & 1 & 0.5 & 1 & 1 \\
\hline$s_{2}^{(0)}$ & 0 & 0 & 0 & 0 & 0 & 0.5 & 0.5 & 0.5 & 1 \\
\hline Tempo [s] & 0.237 & 0.289 & 0.413 & 0.329 & 0.290 & 0.381 & 0.353 & 0.448 & 0.251 \\
\hline Iterações & 2 & 2 & 3 & 2 & 2 & 2 & 2 & 3 & 2 \\
\hline
\end{tabular}

Tabela 6.34 - Resultados para o sistema IEEE de 30 barras. Soluções com * são dominadas pelas demais.

\begin{tabular}{|c|c|c|c|c|c|c|c|c|c|c|}
\hline \multicolumn{11}{|c|}{ Função Senoidal } \\
\hline $\bar{\epsilon}$ & 1 & $2^{*}$ & 3 & 4 & 5 & 6 & 7 & 8 & $9^{*}$ & 10 \\
\hline Perdas $[\mathrm{MW}]$ & 17.46 & 17.46 & 17.19 & 17.12 & 16.27 & 16.16 & 16.06 & 16.04 & 16.06 & 16.03 \\
\hline$\Delta \mathrm{P}[\mathrm{MW}]$ & - & 0.0 & -0.27 & -0.07 & -0.85 & -0.11 & -0.10 & -0.02 & 0.02 & -0.01 \\
\hline$\sum_{k \in \mathcal{B}} s_{1_{k}}$ & 0 & 0 & 3 & 4 & 5 & 6 & 6 & 6 & 5 & 6 \\
\hline$\sum_{k, m \in \mathcal{T}} s_{2_{k m}}$ & 1 & 2 & 0 & 0 & 0 & 0 & 1 & 2 & 4 & 4 \\
\hline$s_{1}^{(0)}$ & 1 & 0 & 1 & 1 & 1 & 1 & 1 & 1 & 1 & 1 \\
\hline$s_{2}^{(0)}$ & 0.5 & 0.5 & 1 & 0 & 0.5 & 0.5 & 0.5 & 0.5 & 1 & 0.5 \\
\hline Tempo $[\mathrm{s}]$ & 0.950 & 0.742 & 0.453 & 0.744 & 0.500 & 0.608 & 0.722 & 1.123 & 1.020 & 0.734 \\
\hline Iterações & 4 & 4 & 2 & 2 & 2 & 2 & 3 & 3 & 4 & 4 \\
\hline \multicolumn{11}{|c|}{ Função Polinomial } \\
\hline $\bar{\epsilon}$ & 1 & 2 & 3 & 4 & 5 & 6 & 7 & 8 & $9^{*}$ & 10 \\
\hline Perdas [MW] & 17.46 & 17.44 & 17.16 & 16.31 & 16.21 & 16.16 & 16.06 & 16.04 & 16.04 & 16.02 \\
\hline$\Delta \mathrm{P}[\mathrm{MW}]$ & - & -0.02 & -0.28 & -0.85 & -0.10 & -0.05 & -0.10 & -0.02 & 0.00 & -0.02 \\
\hline$\sum_{k \in \mathcal{B}} s_{1_{k}}$ & 0 & 0 & 3 & 4 & 5 & 6 & 6 & 6 & 5 & 6 \\
\hline$\sum_{k, m \in \mathcal{T}} s_{2_{k m}}$ & 1 & 2 & 0 & 0 & 0 & 0 & 1 & 2 & 4 & 4 \\
\hline$s_{1}^{(0)}$ & 0 & 1 & 1 & 0.5 & 0.5 & 0.5 & 1 & 1 & 1 & 1 \\
\hline$s_{2}^{(0)}$ & 0 & 1 & 0.5 & 0 & 0 & 0 & 0.5 & 0.5 & 1 & 1 \\
\hline Tempo $[\mathrm{s}]$ & 2.057 & 1.070 & 0.501 & 0.587 & 0.578 & 0.382 & 0.567 & 0.784 & 0.672 & 0.487 \\
\hline Iterações & 4 & 4 & 2 & 2 & 2 & 2 & 2 & 4 & 3 & 3 \\
\hline
\end{tabular}


Tabela 6.35 - Resultados para o sistema IEEE de 57 barras. Soluções com * são dominadas pelas demais.

\begin{tabular}{|c|c|c|c|c|c|c|c|c|c|c|c|}
\hline \multicolumn{12}{|c|}{ Função Senoidal } \\
\hline $\bar{\epsilon}$ & 1 & 2 & 3 & 4 & 5 & $6^{*}$ & 7 & 8 & 9 & $10^{*}$ & 11 \\
\hline Perdas [MW] & 25.90 & 25.69 & 25.32 & 25.04 & 23.86 & 24.03 & 22.78 & 22.74 & 21.68 & 22.72 & 21.54 \\
\hline$\Delta \mathrm{P}[\mathrm{MW}]$ & - & -0.21 & -0.37 & -0.28 & -1.18 & 0.17 & -1.25 & -0.04 & -1.06 & 1.04 & -1.18 \\
\hline$\sum_{k \in \mathcal{B}} s_{1_{k}}$ & 1 & 2 & 3 & 4 & 5 & 6 & 7 & 7 & 7 & 7 & 7 \\
\hline$\sum_{k, m \in \mathcal{T}} s_{2_{k m}}$ & 0 & 0 & 0 & 0 & 0 & 0 & 0 & 1 & 2 & 3 & 4 \\
\hline$s_{1}^{(0)}$ & 1 & 1 & 1 & 1 & 1 & 1 & 1 & 1 & 1 & 1 & 1 \\
\hline$s_{2}^{(0)}$ & 0.5 & 1 & 0.5 & 0.5 & 0.5 & 0.5 & 0.5 & 0.5 & 0.5 & 0 & 0 \\
\hline Tempo $[\mathrm{s}]$ & 1.225 & 1.556 & 0.774 & 0.963 & 1.276 & 1.033 & 0.695 & 1.066 & 1.191 & 0.967 & 1.775 \\
\hline Iterações & 2 & 2 & 2 & 2 & 2 & 2 & 2 & 3 & 4 & 4 & 4 \\
\hline & & & & & & & & & & & \\
\hline$\epsilon$ & 12 & 13 & $14^{*}$ & $15^{*}$ & 16 & 17 & $18^{*}$ & $19^{*}$ & 20 & 21 & $22^{*}$ \\
\hline Perdas [MW] & 21.50 & 21.36 & 21.46 & 21.38 & 21.31 & 21.23 & 21.42 & 21.25 & 21.01 & 21.00 & 21.05 \\
\hline$\Delta \mathrm{P}[\mathrm{MW}]$ & -0.04 & -0.14 & 0.10 & -0.08 & -0.07 & -0.08 & 0.19 & -0.17 & -0.24 & -0.01 & 0.05 \\
\hline$\sum_{k \in \mathcal{B}} s_{1_{k}}$ & 7 & 7 & 7 & 7 & 7 & 7 & 7 & 7 & 7 & 7 & 7 \\
\hline$\sum_{k, m \in \mathcal{T}} s_{2_{k m}}$ & 5 & 6 & 7 & 8 & 9 & 10 & 11 & 12 & 13 & 14 & 15 \\
\hline$s_{1}^{(0)}$ & 1 & 1 & 1 & 0 & 0.5 & 0.5 & 1 & 1 & 1 & 0 & 1 \\
\hline$s_{2}^{(0)}$ & 0 & 0 & 0 & 0 & 0.5 & 0.5 & 0 & 0 & 0 & 0 & 0.5 \\
\hline Tempo $[\mathrm{s}]$ & 1.780 & 1.461 & 1.498 & 2.555 & 2.006 & 1.402 & 2.331 & 2.090 & 1.440 & 3.575 & 1.854 \\
\hline Iterações & 5 & 5 & 4 & 5 & 5 & 5 & 5 & 5 & 5 & 5 & 5 \\
\hline \multicolumn{12}{|c|}{ Função Polinomial } \\
\hline$\epsilon$ & 1 & 2 & 3 & 4 & 5 & $6^{*}$ & 7 & 8 & 9 & 10 & 11 \\
\hline Perdas [MW] & 25.90 & 25.70 & 25.67 & 25.04 & 23.86 & 24.03 & 22.78 & 22.74 & 21.68 & 21.65 & 21.54 \\
\hline$\Delta \mathrm{P}[\mathrm{MW}]$ & - & -0.20 & -0.03 & -0.63 & -1.18 & 0.17 & -1.25 & -0.04 & -1.06 & -0.03 & -0.11 \\
\hline$\sum_{k \in \mathcal{B}} s_{1_{k}}$ & 1 & 1 & 3 & 4 & 5 & 6 & 7 & 7 & 7 & 7 & 7 \\
\hline$\sum_{k, m \in \mathcal{T}} s_{2_{k m}}$ & 0 & 1 & 0 & 0 & 0 & 0 & 0 & 1 & 2 & 3 & 4 \\
\hline$s_{1}^{(0)}$ & 1 & 1 & 1 & 1 & 1 & 1 & 1 & 1 & 1 & 0.5 & 1 \\
\hline$s_{2}^{(0)}$ & 0.5 & 0.5 & 0.5 & 0.5 & 0.5 & 0.5 & 0.5 & 0.5 & 0.5 & 0 & 0 \\
\hline Tempo $[\mathrm{s}]$ & 1.234 & 1.393 & 1.083 & 0.566 & 1.091 & 0.687 & 0.542 & 1.133 & 0.688 & 1.247 & 1.327 \\
\hline Iterações & 2 & 3 & 2 & 2 & 2 & 2 & 2 & 2 & 2 & 3 & 4 \\
\hline$\epsilon$ & 12 & 13 & $14^{*}$ & 15 & 16 & $17^{*}$ & $18^{*}$ & $19^{*}$ & 20 & 21 & $22^{*}$ \\
\hline Perdas [MW] & 21.45 & 21.37 & 21.46 & 21.29 & 21.12 & 21.21 & 21.28 & 21.16 & 21.02 & 20.99 & 21.00 \\
\hline$\Delta \mathrm{P}[\mathrm{MW}]$ & -0.09 & -0.08 & 0.09 & -0.17 & -0.17 & 0.09 & 0.07 & -0.12 & -0.14 & -0.03 & 0.01 \\
\hline$\sum_{k \in \mathcal{B}} s_{1_{k}}$ & 7 & 7 & 7 & 7 & 7 & 7 & 7 & 7 & 7 & 7 & 7 \\
\hline$\sum_{k, m \in \mathcal{T}} s_{2_{k m}}$ & 5 & 6 & 7 & 8 & 9 & 10 & 11 & 12 & 13 & 14 & 15 \\
\hline$s_{1}^{(0)}$ & 0.5 & 1 & 1 & 0.5 & 0.5 & 0.5 & 0.5 & 1 & 0 & 1 & 1 \\
\hline$s_{2}^{(0)}$ & 0 & 0 & 0 & 0.5 & 0.5 & 0 & 0 & 0 & 0 & 0 & 0 \\
\hline Tempo [s] & 1.678 & 1.323 & 1.395 & 1.460 & 1.948 & 1.404 & 1.424 & 1.331 & 2.114 & 1.618 & 1.282 \\
\hline Iterações & 3 & 4 & 4 & 4 & 5 & 4 & 5 & 5 & 5 & 4 & 6 \\
\hline
\end{tabular}


Tabela 6.36 - Resultados para o sistema IEEE de 118 barras. Somente com soluções não dominadas.

\begin{tabular}{|c|c|c|c|c|c|c|c|c|c|c|c|}
\hline \multicolumn{12}{|c|}{ Função Senoidal } \\
\hline $\bar{\epsilon}$ & 5 & 11 & 20 & 25 & 35 & 40 & 45 & 50 & 56 & 60 & 61 \\
\hline Perdas [MW] & 132.14 & 132.13 & 130.56 & 128.68 & 124.60 & 124.32 & 122.35 & 118.21 & 106.92 & 106.57 & 106.15 \\
\hline$\Delta \mathrm{P}[\mathrm{MW}]$ & - & -0.01 & -1.57 & -1.88 & -4.08 & -0.28 & -1.97 & -4.14 & -11.29 & -0.35 & -0.42 \\
\hline$\sum_{k \in \mathcal{B}} s_{1_{k}}$ & 5 & 11 & 19 & 25 & 33 & 31 & 45 & 50 & 54 & 54 & 54 \\
\hline$\sum_{k, m \in \mathcal{T}} s_{2_{k m}}$ & 0 & 0 & 1 & 0 & 2 & 9 & 0 & 0 & 2 & 6 & 7 \\
\hline$s_{1}^{(0)}$ & 1 & 1 & 1 & 1 & 0 & 0 & 1 & 1 & 1 & 1 & 1 \\
\hline$s_{2}^{(0)}$ & 1 & 0 & 0.5 & 0 & 0 & 0 & 0 & 0 & 0 & 0.5 & 0 \\
\hline Tempo [s] & 4.681 & 3.549 & 1.310 & 1.719 & 1.672 & 2.342 & 2.544 & 2.494 & 2.132 & 2.200 & 2.424 \\
\hline Iterações & 2 & 2 & 2 & 2 & 3 & 4 & 2 & 2 & 4 & 3 & 3 \\
\hline \multicolumn{12}{|c|}{ Função Polinomial } \\
\hline$\epsilon$ & 5 & 9 & 12 & 25 & 35 & 40 & 45 & 50 & 56 & 59 & 63 \\
\hline Perdas [MW] & 131.65 & 131.13 & 130.63 & 128.56 & 123.53 & 122.76 & 119.66 & 113.53 & 107.24 & 106.60 & 106.10 \\
\hline$\Delta \mathrm{P}[\mathrm{MW}]$ & - & -0.52 & -0.50 & -2.07 & -5.03 & -0.77 & -3.10 & -6.13 & -6.29 & -0.64 & -0.50 \\
\hline$\sum_{k \in \mathcal{B}} s_{1_{k}}$ & 4 & 8 & 11 & 25 & 35 & 40 & 45 & 50 & 54 & 54 & 54 \\
\hline$\sum_{k, m \in \mathcal{T}} s_{2 k m}$ & 1 & 1 & 1 & 0 & 0 & 0 & 0 & 0 & 2 & 5 & 9 \\
\hline$s_{1}^{(0)}$ & 0 & 1 & 0.5 & 0.5 & 0.5 & 0.5 & 0.5 & 0 & 1 & 0 & 1 \\
\hline$s_{2}^{(0)}$ & 0 & 1 & 0.5 & 0.5 & 0 & 0.5 & 0.5 & 0 & 0 & 0 & 0.5 \\
\hline Tempo $[\mathrm{s}]$ & 11.846 & 7.403 & 14.049 & 5.215 & 2.615 & 1.980 & 1.973 & 3.649 & 2.591 & 3.915 & 2.285 \\
\hline Iterações & 6 & 3 & 6 & 2 & 2 & 2 & 2 & 2 & 3 & 4 & 4 \\
\hline
\end{tabular}

As Figuras 6.39, 6.40, 6.41, 6.42 e 6.43 apresentam o trade-off entre as perdas ativas e o número de ações de controle para os sistemas IEEE de 14, 30, 57, 118 e 300 barras, respectivamente.

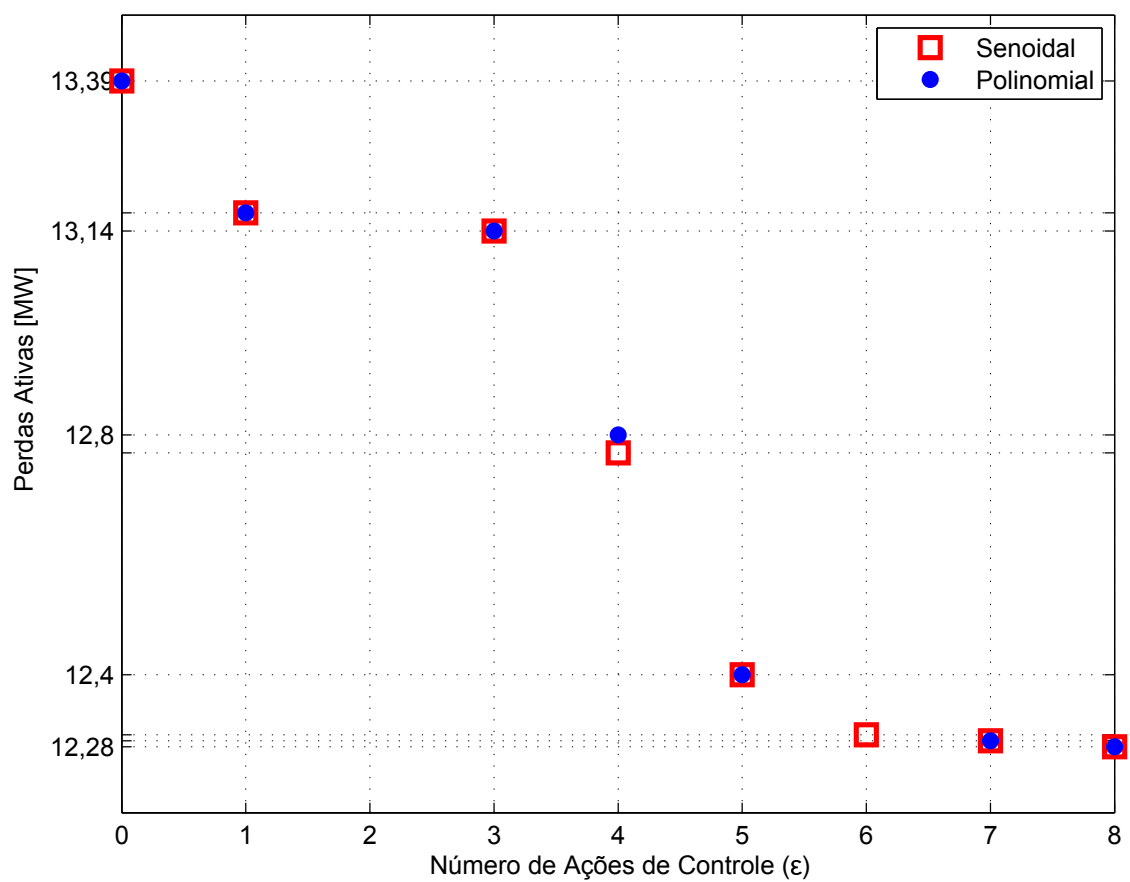

Figura 6.39 - Trade-off entre as perdas ativas e o número de ações de controle para o sistema IEEE de 14 barras. 


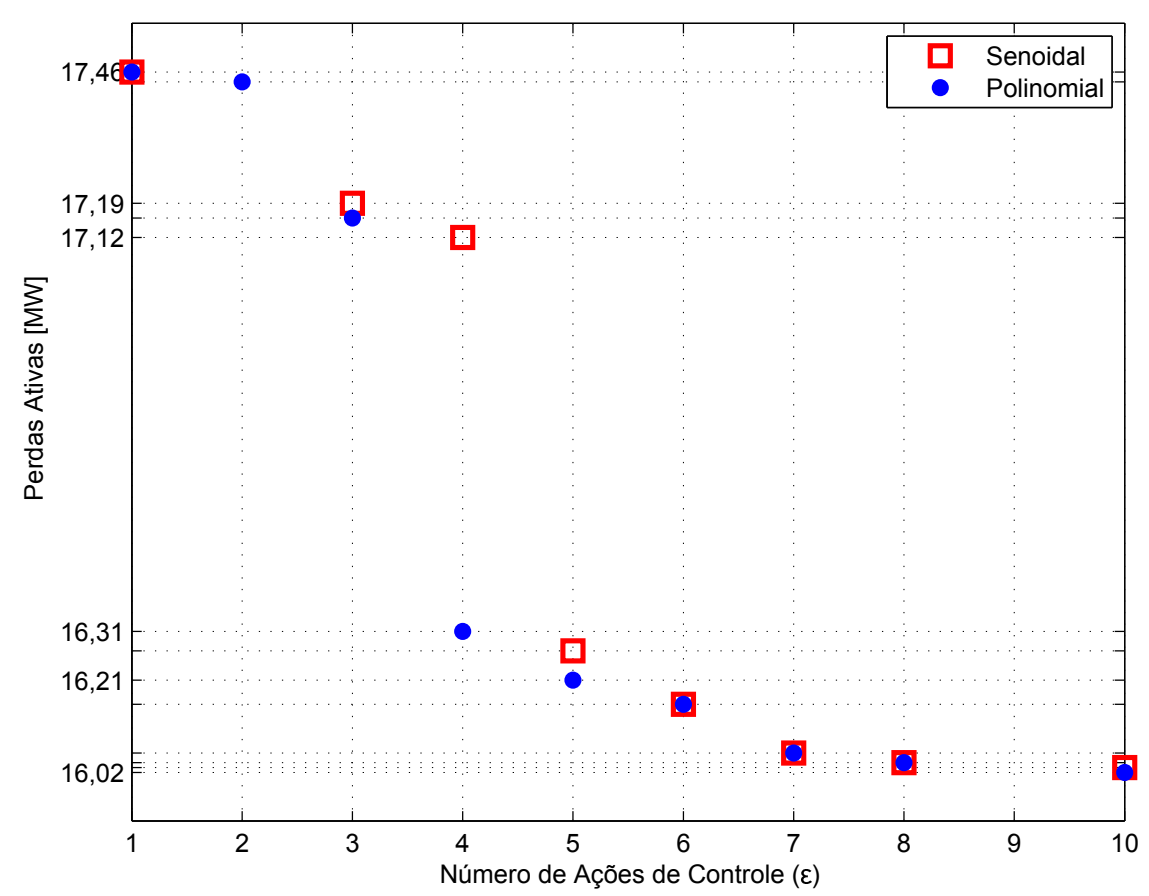

Figura 6.40 - Trade-off entre as perdas ativas e o número de ações de controle para o sistema IEEE de 30 barras.

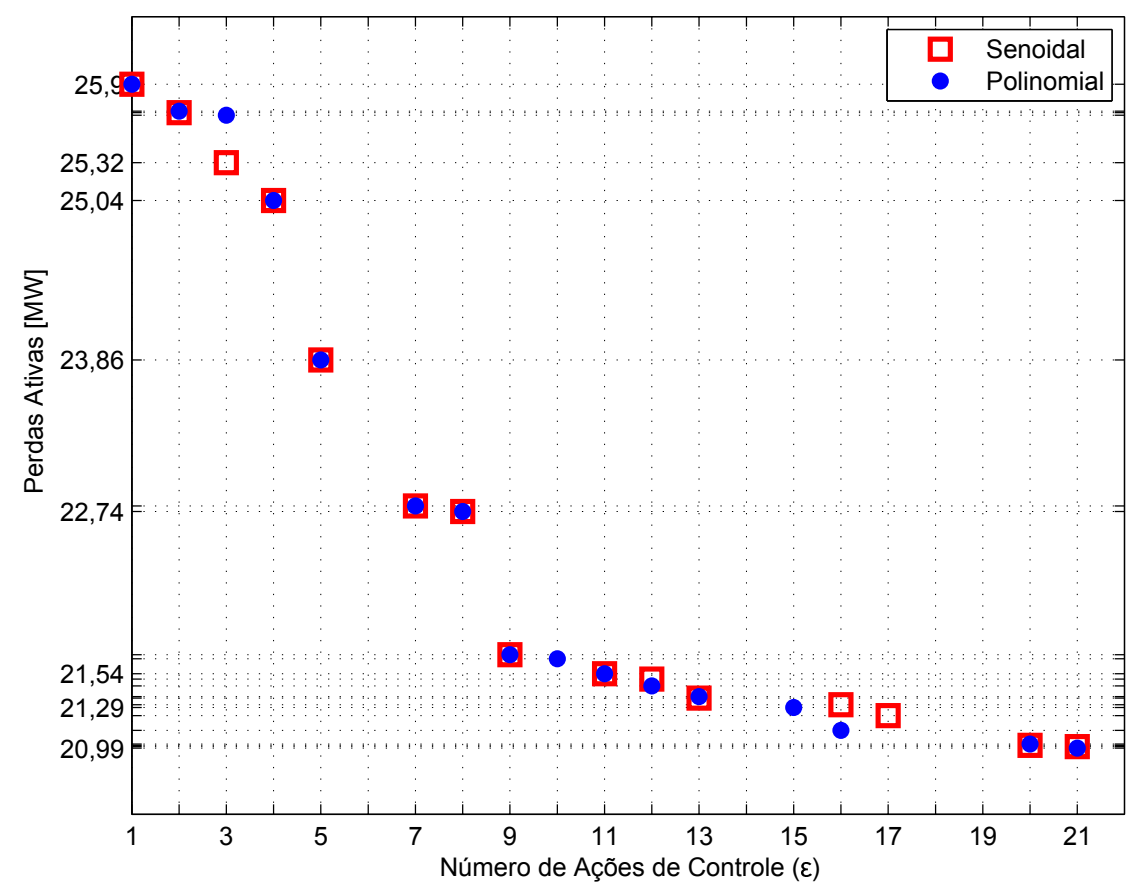

Figura 6.41 - Trade-off entre as perdas ativas e o número de ações de controle para o sistema IEEE de 57 barras. 


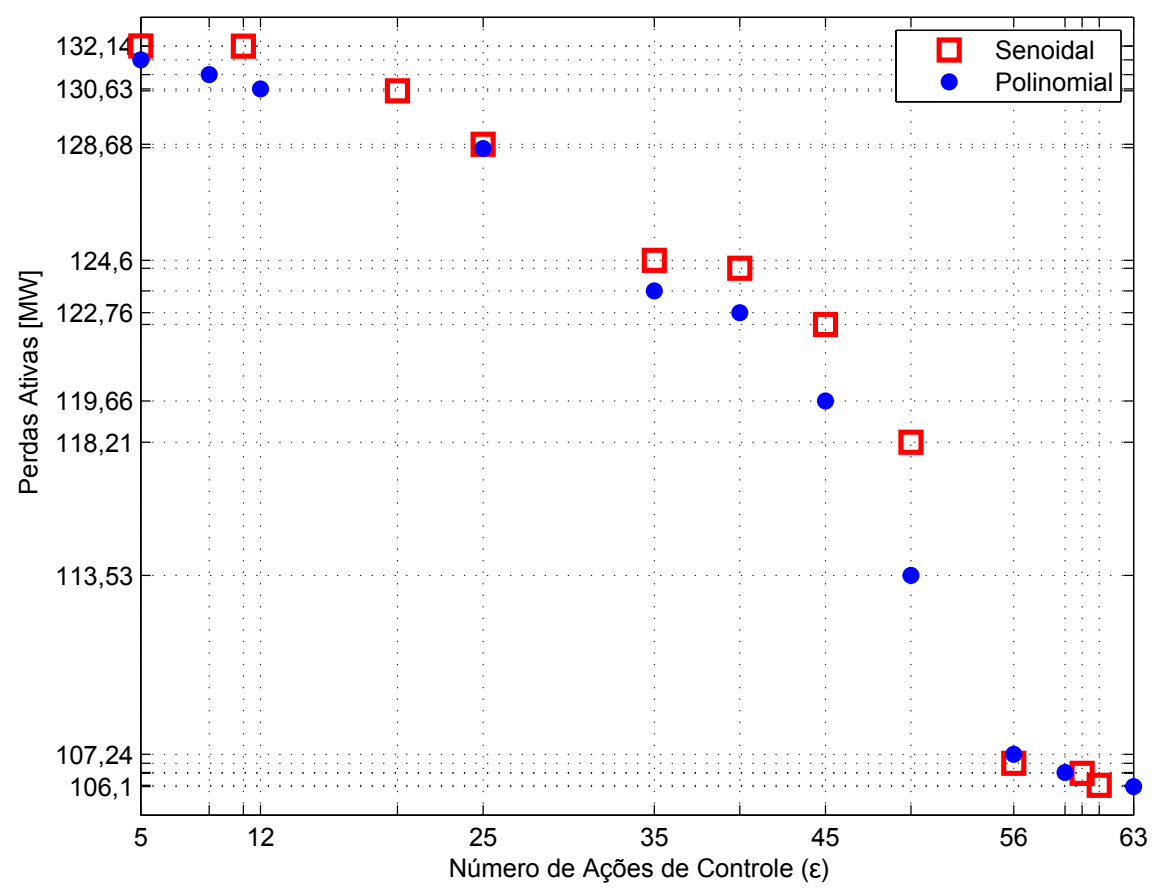

Figura 6.42 - Trade-off entre as perdas ativas e o número de ações de controle para o sistema IEEE de 118 barras.

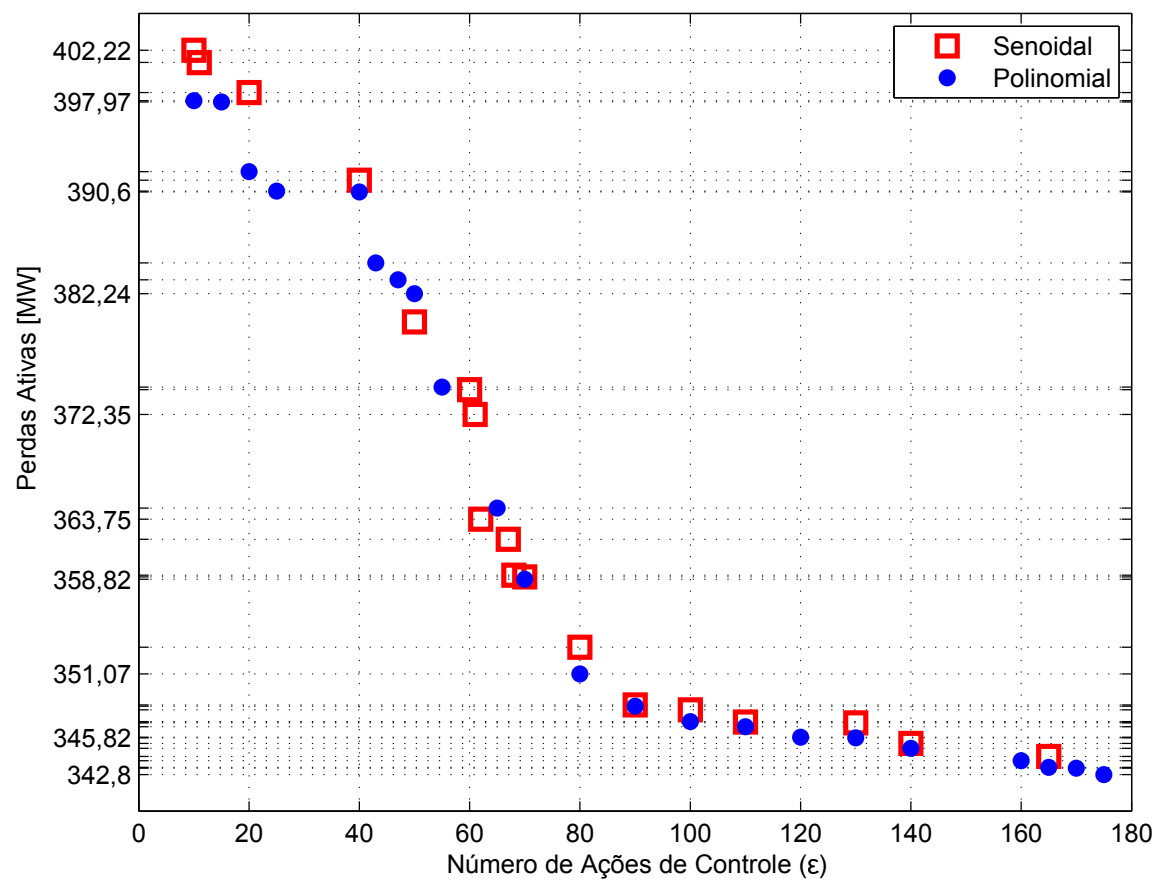

Figura 6.43 - Trade-off entre as perdas ativas e o número de ações de controle para o sistema IEEE de 300 barras. 
Tabela 6.37 - Resultados para o sistema IEEE de 300 barras. Somente com soluções não dominadas.

\begin{tabular}{|c|c|c|c|c|c|c|c|c|c|c|c|}
\hline \multicolumn{12}{|c|}{ Função Senoidal } \\
\hline $\bar{\epsilon}$ & 10 & 11 & 20 & 40 & 50 & 60 & 61 & 62 & 67 & 68 & 70 \\
\hline Perdas [MW] & 402.22 & 401.20 & 398.74 & 391.55 & 379.94 & 374.38 & 372.35 & 363.75 & 362.11 & 359.16 & 359.02 \\
\hline$\Delta$ Perdas $[\mathrm{MW}]$ & - & -1.02 & -2.46 & -7.19 & -11.61 & -5.56 & -2.03 & -8.60 & -1.64 & -2.95 & -0.14 \\
\hline$\sum_{k \in \mathcal{B}} s_{1_{k}}$ & 3 & 6 & 5 & 16 & 50 & 60 & 61 & 62 & 67 & 68 & 69 \\
\hline$\sum_{k, m \in \mathcal{T}} s_{2_{k m}}$ & 7 & 5 & 15 & 24 & 0 & 0 & 0 & 0 & 0 & 0 & 1 \\
\hline$s_{1}^{(0)}$ & 0 & 0 & 1 & 0.5 & 1 & 1 & 1 & 1 & 1 & 1 & 1 \\
\hline$s_{2}^{(0)}$ & 0.5 & 0 & 0 & 0.5 & 0 & 0.5 & 0.5 & 0.5 & 0.5 & 0.5 & 0.5 \\
\hline Tempo [s] & 11.304 & 8.395 & 17.875 & 22.069 & 5.269 & 5.251 & 5.773 & 4.450 & 7.780 & 21.288 & 9.159 \\
\hline Iterações & 5 & 5 & 5 & 6 & 2 & 2 & 2 & 2 & 2 & 2 & 4 \\
\hline$\epsilon$ & 80 & 90 & 100 & 110 & 130 & 140 & 165 & & & & \\
\hline Perdas [MW] & 353.25 & 348.52 & 348.12 & 347.12 & 347.04 & 345.36 & 344.30 & & & & \\
\hline$\Delta \mathrm{P}[\mathrm{MW}]$ & -5.77 & -4.73 & -0.40 & -1.00 & -0.08 & -1.68 & -1.06 & & & & \\
\hline$\sum_{k \in \mathcal{B}} s_{1_{k}}$ & 69 & 69 & 69 & 69 & 69 & 69 & 69 & & & & \\
\hline$\sum_{k, m \in \mathcal{T}} s_{2_{k m}}$ & 11 & 21 & 31 & 41 & 61 & 71 & 96 & & & & \\
\hline$s_{1}^{(0)}$ & 1 & 1 & 1 & 1 & 1 & 1 & 1 & & & & \\
\hline$s_{2}^{(0)}$ & 0 & 0.5 & 0 & 0.5 & 0.5 & 0 & 0.5 & & & & \\
\hline Tempo $[\mathrm{s}]$ & 10.130 & 11.708 & 17.588 & 14.902 & 15.262 & 42.364 & 18.849 & & & & \\
\hline Iterações & 5 & 5 & 6 & 5 & 6 & 6 & 5 & & & & \\
\hline \multicolumn{12}{|c|}{ Função Polinomial } \\
\hline$\epsilon$ & 10 & 15 & 20 & 25 & 40 & 43 & 47 & 50 & 55 & 65 & 70 \\
\hline Perdas [MW] & 398.07 & 397.97 & 392.26 & 390.67 & 390.60 & 384.77 & 383.39 & 382.24 & 374.58 & 364.67 & 358.82 \\
\hline$\Delta$ Perdas $[\mathrm{MW}]$ & - & -0.10 & -5.71 & -1.59 & -0.07 & -5.83 & -1.38 & -1.15 & -7.66 & -9.91 & -5.85 \\
\hline$\sum_{k \in \mathcal{B}} s_{1_{k}}$ & 10 & 3 & 7 & 12 & 40 & 40 & 47 & 49 & 41 & 65 & 69 \\
\hline$\sum_{k, m \in \mathcal{T}} s_{2 k m}$ & 0 & 12 & 13 & 13 & 0 & 3 & 0 & 1 & 14 & 0 & 1 \\
\hline$s_{1}^{(0)}$ & 1 & 1 & 1 & 1 & 1 & 0 & 0.5 & 0 & 1 & 1 & 0.5 \\
\hline$s_{2}^{(0)}$ & 0.5 & 1 & 1 & 1 & 0 & 0 & 1 & 0 & 1 & 0 & 0 \\
\hline Tempo $[\mathrm{s}]$ & 5.498 & 15.353 & 9.299 & 11.802 & 3.257 & 24.183 & 5.151 & 22.306 & 27.461 & 6.311 & 7.905 \\
\hline Iterações & 2 & 6 & 7 & 7 & 2 & 6 & 2 & 6 & 6 & 2 & 3 \\
\hline$\epsilon$ & 80 & 00 & 100 & 110 & 120 & $\sqrt{13}$ & 140 & 16 & 16 & 170 & 175 \\
\hline$\frac{\epsilon}{\text { Perdas [MW] }}$ & 351.07 & 348.41 & 347.16 & 346.73 & $\frac{120}{345.87}$ & $\begin{array}{c}150 \\
345.82\end{array}$ & $\begin{array}{c}140 \\
344.96\end{array}$ & $\begin{array}{c}100 \\
343.94\end{array}$ & $\frac{165}{343.40}$ & $\frac{170}{343.34}$ & $\frac{175}{342.80}$ \\
\hline$\Delta \mathrm{P}[\mathrm{MW}]$ & -7.75 & -2.66 & -1.25 & -0.43 & -0.86 & -0.05 & -0.86 & -1.02 & -0.54 & -0.06 & -0.54 \\
\hline$\sum_{k \in \mathcal{B}} s_{1_{k}}$ & 69 & 69 & 69 & 69 & 69 & 69 & 69 & 69 & 69 & 69 & 69 \\
\hline$\sum_{k, m \in \mathcal{T}} s_{2_{k m}}$ & 11 & 21 & 31 & 41 & 51 & 61 & 71 & 91 & 96 & 101 & 106 \\
\hline$s_{1}^{(0)}$ & 1 & 1 & 1 & 1 & 1 & 1 & 1 & 1 & 1 & 0.5 & 0.5 \\
\hline$s_{2}^{(0)}$ & 0 & 0.5 & 0 & 0 & 0 & 0 & 0 & 0.5 & 0.5 & 0 & 0 \\
\hline Tempo $[\mathrm{s}]$ & 6.942 & 11.097 & 12.666 & 13.013 & 13.074 & 15.767 & 12.054 & 12.844 & 14.494 & 24.313 & 11.643 \\
\hline Iterações & 4 & 6 & 4 & 6 & 6 & 6 & 5 & 6 & 6 & 6 & 6 \\
\hline
\end{tabular}




\subsubsection{Análise dos Resultados}

Para o sistema de 14 barras, as funções senoidal e polinomial (Algoritmos 1 e 2) encontraram soluções para os nove possíveis valores de $\epsilon$. As duas funções chegaram em sete soluções compatíveis entre si, sendo que cinco destas se encontram no mesmo ótimo local A Tabela 6.38 apresenta os resultados para $\epsilon=7$ e $\epsilon=8$ para ambas as funções de discretização, ponto a ponto. Observe que para $\epsilon=7$ as funções senoidal e polinomial chegam as mesmas perdas ativas com diferentes ótimos locais, o mesmo ocorre para $\epsilon=8$. Para $\epsilon=4$ e $\epsilon=6$ a função senoidal obteve um melhor resultado quando comparada com a função polinomial. Em todos os casos as funções de discretização encontram resultados discretos factíveis.

Tabela 6.38 - Tensões e taps de transformadores para os $\epsilon$ 's 7 e 8 do sistema IEEE de 14 barras.

\begin{tabular}{c|c|c|c|c}
\hline$\epsilon$ & \multicolumn{2}{|c|}{7} & \multicolumn{2}{c}{8} \\
\hline Variáveis & Senoidal & Polinomial & Senoidal & Polinomial \\
$V_{1}$ & 1.1000 & 1.1000 & 1.1000 & 1.1000 \\
$V_{2}$ & 1.0857 & 1.0858 & 1.0860 & 1.0859 \\
$V_{3}$ & 1.0562 & 1.0562 & 1.0567 & 1.0566 \\
$V_{6}$ & 1.0962 & 1.0964 & 1.0991 & 1.0998 \\
$V_{8}$ & 1.0900 & 1.0900 & 1.1000 & 1.1000 \\
$t_{4-7}$ & 1.0450 & 1.0525 & 1.0450 & 1.0450 \\
$t_{4-9}$ & 0.8950 & 0.8875 & 0.8950 & 0.8875 \\
$t_{5-6}$ & 0.9775 & 0.9775 & 0.9775 & 0.9775 \\
\hline
\end{tabular}

Dos dez $\epsilon$ testados para o sistema de 30 barras apenas quatro encontraram o mesmo valor para perdas entre senoidal e polinomial, e desses apenas $\epsilon=6$ encontra o mesmo mínimo local. A função senoidal possui duas soluções dominadas enquanto a polinomial apenas uma. A função polinomial domina a função senoidal em cinco soluções, e as demais são equivalentes.

Para o sistema de 57 barras, foram testados vinte e dois valores para $\epsilon$, a função senoidal encontra quinze soluções não dominadas enquanto a polinomial encontra dezesseis. Considerando todos os valores, a função senoidal domina a polinomial em apenas quatro soluções, já a polinomial domina a senoidal em nove casos, sete soluções foram iguais em desempenho para as funções de discretização.

Para o sistema de 118 barras, testamos vinte e sete valores para $\epsilon$. Os resultados apresentados na Tabela 6.36 são as soluções não dominadas encontradas para cada função de discretização. A Figura 6.44 apresenta a curva combinada de ambas funções de discretização descartando as soluções dominadas entre si.

Para o sistema de 300 barras foram testados quarenta e cinco valores para $\epsilon$. Os resultados apresentados na Tabela 6.37 são as soluções não dominadas encontradas para cada função de discretização. A função senoidal encontrou um número menor de soluções factíveis do que a polinomial, em muitos casos a senoidal não conseguiu discretizar, esse problema não 


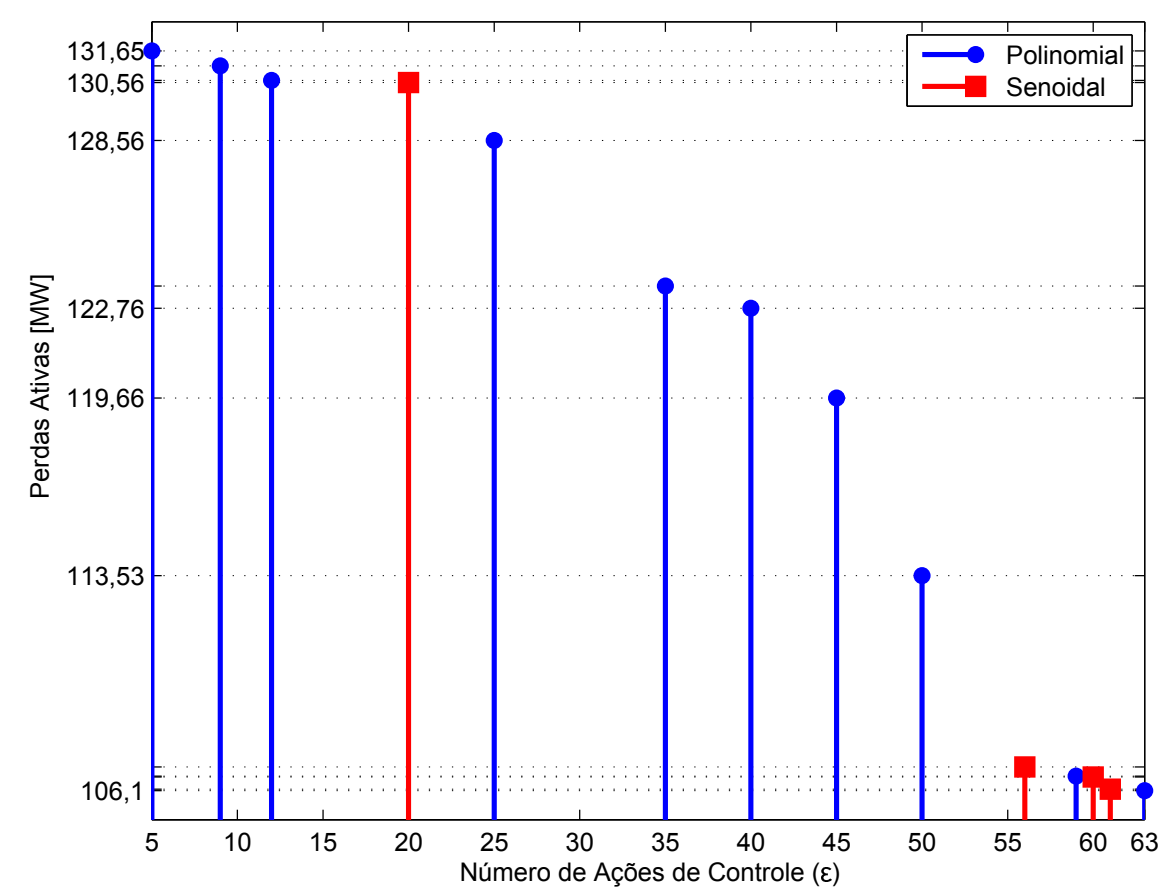

Figura 6.44 - Perdas ativas pelo número de ações de controle para o sistema de 118 barras. Soluções não dominadas geradas pela combinação das soluções das funções de discretização.

ocorreu com frequência para a função polinomial. A Figura 6.45 apresenta a curva combinada de ambas funções de discretização descartando as soluções dominadas entre si. Verificou-se que a função polinomial tem dezoito soluções na curva combinada, enquanto a senoidal possui apenas seis soluções.

\subsubsection{Conclusões}

Nesta seção, apresentamos resultados para o problema de FPO multiobjetivo com tap de transformadores discretos e bancos de capacitores e reatores shunts fixos. Buscava-se verificar a eficiência das funções de discretização em encontrar soluções discretas factíveis para um problema multiobjetivo. O problema de FPO multiobjetivo foi modificado pelo método $\epsilon$-restrito, passando a ser tratado como um problema mono-objetivo. As variáveis binárias associadas aos controles foram tratadas por uma função quadrática, enquanto as variáveis discretas foram tratadas por duas funções de discretização. Bons resultados foram obtidos utilizando os Algoritmos 1 e 2, juntamente o solver IPOPT, para a resolução do problema modificado (5.9). A estratégia utilizando o método $\epsilon$-restrito, a função quadrática e as funções de discretização, é eficiente chegando a uma solução factível para o problema. Fica evidente que a função de discretização polinomial encontra, em geral, melhores soluções para o problema se comparada com a função de discretização senoidal. 


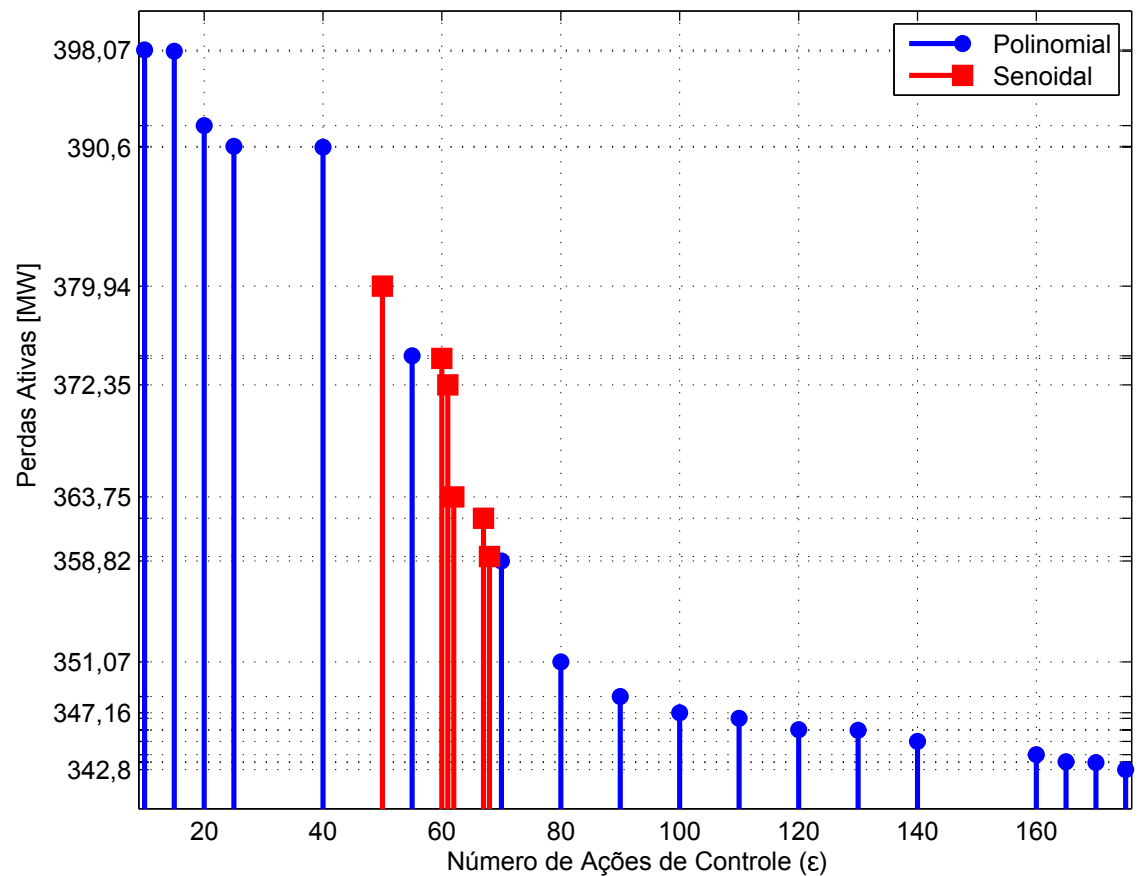

Figura 6.45 - Perdas ativas pelo número de ações de controle para o sistema de 300 barras. Soluções não dominadas geradas pela combinação das soluções das funções de discretização.

A próxima subseção contém os resultados para o problema de FPO multiobjetivo com taps de transformadores discretos e banco de capacitores e reatores shunts discretos é modelado pelo método da soma ponderada. As variáveis discretas são tratadas pelas funções de discretização e as variáveis binárias pela função Sigmoidal. 


\subsubsection{FPO Multiobjetivo com Tap de Transformadores e Susceptâncias Shunts Discretos}

O modelo de FPO Multiobjetivo com taps de transformadores e os bancos de capacitores e reatores shunts discretos (2.15) é modificado pelo método da soma ponderada com em (5.10), deixando de ser um problema multiobjetivo para um PNL com variáveis contínuas, discretas e binárias. As variáveis binárias do problema (5.10) são modificadas pela função Sigmoidal como em (5.11). O problema (5.11) possui variáveis discretas que serão tratadas pelas funções de discretização Senoidal, Polinomial e Mista descritas no Capítulo 3.

A Tabela 6.39 mostra as características de cada sistema para o modelo de FPO multiobjetivo (2.15) com tap de transformadores e bancos de capacitores e reatores shunts discretos.

Tabela 6.39 - Características dos Sistemas Elétricos

\begin{tabular}{c|ccccc}
\hline $\begin{array}{c}\text { Sistema } \\
\text { Elétrico }\end{array}$ & $\begin{array}{c}\text { Restrições de } \\
\text { Igualdade }\end{array}$ & $\begin{array}{c}\text { Variáveis } \\
\text { Contínuas }\end{array}$ & $\begin{array}{c}\text { Variáveis } \\
\text { Discretas }\end{array}$ & $\begin{array}{c}\text { Variáveis } \\
\text { de Controle }\end{array}$ & $\begin{array}{c}\text { Variáveis } \\
\text { Binárias }\end{array}$ \\
\hline 14 & 22 & 27 & 4 & 9 & 9 \\
30 & 53 & 59 & 6 & 12 & 12 \\
57 & 106 & 113 & 18 & 25 & 25 \\
118 & 181 & 235 & 23 & 77 & 77 \\
300 & 530 & 599 & 121 & 190 & 190 \\
\hline
\end{tabular}

Inicialmente resolver-se o problema (5.13) que considera as variáveis taps de transformadores e os bancos de capacitores e reatores shunts contínuas e posteriormente discretiza-se essas variáveis utilizando as funções de discretização apresentadas. O Algoritmo 7 descreve todo o processo para encontrar uma solução discreta factível para o problema de FPO Multiobjetivo com taps de transformadores e os bancos de capacitores e reatores shunts discretos (2.15).

O número de iterações apresentado nas tabelas esta separado em dois tipos: IterSIG e Iteração. O valor de IterSIG representa um contador interno do Algoritmo 6, que utiliza a função sigmoidal, e consiste na primeira fase da solução do problema e está relacionado às decisões dos controles. O valor de Iteração está relacionado a segunda fase do Algoritmo 7, que representa a fase de discretização. Ou seja IterSIG é o número de iterações do Algoritmo 6 e Iteração é número de iterações realizadas na discretização da segunda fase do Algoritmo 7.

As Tabelas 6.40, 6.41, 6.42, 6.43 e 6.44 apresentam os resultados obtidos para os sistemas IEEE de 14, 30, 57, 118 e 300 barras, respectivamente. Cada tabela apresenta um resultado parcial utilizando o Algoritmo 6 que resolve o problema (5.13) considerando as variáveis discretas como contínuas. Esse resultado parcial é igual para todas as funções de discretização. 
Tabela 6.40 - Resultados do sistema IEEE de 14 barras para os diferentes valores de $\omega$ usando a SIGMOIDAL.

\begin{tabular}{|c|c|c|c|c|c|c|c|c|c|c|c|}
\hline$\omega$ & 0.0 & 0.1 & 0.2 & 0.3 & 0.4 & 0.5 & 0.6 & 0.7 & 0.8 & 0.9 & 1.0 \\
\hline \multicolumn{12}{|c|}{ Resultado Parcial com o Algoritmo 6 usando a SIGMOIDAL } \\
\hline $\mathrm{N}$ & 0 & 0 & 0 & 3 & 4 & 4 & 4 & 4 & 5 & 6 & 9 \\
\hline Perdas $[\mathrm{MW}]$ & 13.39 & 13.39 & 13.39 & 12.57 & 12.34 & 12.34 & 12.34 & 12.34 & 12.31 & 12.28 & 12.27 \\
\hline$\Delta \mathrm{N}$ & - & 0 & 0 & 3 & 1 & 0 & 0 & 0 & 1 & 1 & 3 \\
\hline$\Delta \mathrm{P}[\mathrm{MW}]$ & - & 0 & 0 & -0.82 & -0.23 & 0 & 0 & 0 & -0.03 & -0.03 & -0.01 \\
\hline IterSIG & 2 & 2 & 2 & 4 & 4 & 4 & 4 & 4 & 4 & 4 & 4 \\
\hline Tensão & 0 & 0 & 0 & 3 & 3 & 3 & 3 & 3 & 3 & 4 & 5 \\
\hline Tap & 0 & 0 & 0 & 0 & 1 & 1 & 1 & 1 & 2 & 1 & 3 \\
\hline Shunt & 0 & 0 & 0 & 0 & 0 & 0 & 0 & 0 & 0 & 1 & 1 \\
\hline \multicolumn{12}{|c|}{ Resultado Final com o Algoritmo 7 usando a SENOIDAL } \\
\hline $\mathrm{N}$ & 0 & 0 & 0 & 3 & 4 & 4 & 4 & $\overline{4}$ & $\overline{5}$ & $\overline{7}$ & 9 \\
\hline Perdas $[\mathrm{MW}]$ & 13.39 & 13.39 & 13.39 & 12.57 & 12.34 & 12.35 & 12.34 & 12.34 & 12.31 & 12.28 & 12.27 \\
\hline$\Delta \mathrm{N}$ & - & 0 & 0 & 3 & 1 & 0 & 0 & 0 & 1 & 2 & 2 \\
\hline$\Delta \mathrm{P}[\mathrm{MW}]$ & - & 0 & 0 & -0.82 & -0.23 & 0.01 & -0.01 & 0 & -0.03 & -0.03 & -0.01 \\
\hline Iteração & 2 & 2 & 2 & 2 & 4 & 4 & 2 & 4 & 3 & 6 & 4 \\
\hline Tensão & 0 & 0 & 0 & 3 & 3 & 3 & 3 & 3 & 3 & 5 & 5 \\
\hline Tap & 0 & 0 & 0 & 0 & 1 & 1 & 1 & 1 & 2 & 1 & 3 \\
\hline Shunt & 0 & 0 & 0 & 0 & 0 & 0 & 0 & 0 & 0 & 1 & 1 \\
\hline Tempo (s) & 0.943 & 0.396 & 0.394 & 0.903 & 1.123 & 1.117 & 0.994 & 1.095 & 0.902 & 1.399 & 1.112 \\
\hline \multicolumn{12}{|c|}{ Resultado Final com o Algoritmo 7 usando a POLINOMIAL } \\
\hline $\mathrm{N}$ & 0 & 0 & 0 & 3 & 4 & 4 & 4 & 4 & 5 & 7 & 9 \\
\hline Perdas $[\mathrm{MW}]$ & 13.39 & 13.39 & 13.39 & 12.57 & 12.34 & 12.34 & 12.34 & 12.34 & 12.31 & 12.28 & 12.27 \\
\hline$\Delta \mathrm{N}$ & - & 0 & 0 & 3 & 1 & 0 & 0 & 0 & 1 & 2 & 2 \\
\hline$\Delta \mathrm{P}[\mathrm{MW}]$ & - & 0 & 0 & -0.82 & -0.23 & 0 & 0 & 0 & -0.03 & -0.03 & -0.01 \\
\hline Iteração & 2 & 2 & 2 & 2 & 4 & 4 & 4 & 4 & 2 & 6 & 4 \\
\hline Tensão & 0 & 0 & 0 & 3 & 3 & 3 & 3 & 3 & 3 & 5 & 5 \\
\hline Tap & 0 & 0 & 0 & 0 & 1 & 1 & 1 & 1 & 2 & 1 & 3 \\
\hline Shunt & 0 & 0 & 0 & 0 & 0 & 0 & 0 & 0 & 0 & 1 & 1 \\
\hline Tempo (s) & 0.393 & 0.390 & 0.403 & 0.654 & 1.012 & 1.072 & 1.005 & 1.035 & 0.781 & 1.437 & 0.994 \\
\hline \multicolumn{12}{|c|}{ Resultado Final com o Algoritmo 7 usando a MISTA } \\
\hline$\overline{\mathrm{N}}$ & 0 & 0 & 0 & 3 & 4 & 4 & 4 & 4 & 5 & 7 & 9 \\
\hline Perdas $[\mathrm{MW}]$ & 13.39 & 13.39 & 13.39 & 12.57 & 12.34 & 12.35 & 12.34 & 12.34 & 12.31 & 12.28 & 12.27 \\
\hline$\Delta \mathrm{N}$ & - & 0 & 0 & 3 & 1 & 0 & 0 & 0 & 1 & 2 & 2 \\
\hline$\Delta \mathrm{P}[\mathrm{MW}]$ & - & 0 & 0 & -0.82 & -0.23 & 0.01 & -0.01 & 0 & -0.03 & -0.03 & -0.01 \\
\hline Iteração & 2 & 2 & 2 & 2 & 4 & 4 & 4 & 4 & 3 & 5 & 4 \\
\hline Tensão & 0 & 0 & 0 & 3 & 3 & 3 & 3 & 3 & 3 & 5 & 5 \\
\hline Tap & 0 & 0 & 0 & 0 & 1 & 1 & 1 & 1 & 2 & 1 & 3 \\
\hline Shunt & 0 & 0 & 0 & 0 & 0 & 0 & 0 & 0 & 0 & 1 & 1 \\
\hline Tempo (s) & 0.396 & 0.384 & 0.392 & 0.900 & 0.978 & 1.007 & 1.000 & 0.988 & 0.773 & 1.007 & 0.916 \\
\hline
\end{tabular}


Tabela 6.41 - Resultados do sistema IEEE de 30 barras para os diferentes valores de $\omega$.

\begin{tabular}{|c|c|c|c|c|c|c|c|c|c|c|c|}
\hline \multicolumn{12}{|c|}{ Resultado Parcial com o Algoritmo 6 usando a SIGMOIDAL } \\
\hline$\omega$ & 0.0 & 0.1 & 0.2 & 0.3 & 0.4 & 0.5 & 0.6 & 0.7 & 0.8 & 0.9 & 1.0 \\
\hline $\mathrm{N}$ & 0 & 0 & 0 & 4 & 4 & 6 & 6 & 7 & 8 & 9 & 12 \\
\hline Perdas $[\mathrm{MW}]$ & 17.54 & 17.54 & 17.54 & 16.31 & 16.31 & 16.08 & 16.08 & 16.04 & 16.01 & 15.98 & 15.98 \\
\hline$\Delta \mathrm{N}$ & - & 0 & 0 & 4 & 0 & 2 & 0 & 1 & 1 & 1 & 3 \\
\hline$\Delta \mathrm{P}[\mathrm{MW}]$ & - & 0 & 0 & -1.23 & 0 & -0.23 & 0 & -0.04 & -0.03 & -0.03 & 0 \\
\hline IterSIG & 2 & 2 & 2 & 4 & 4 & 4 & 4 & 4 & 4 & 4 & 5 \\
\hline Tensão & 0 & 0 & 0 & 4 & 4 & 5 & 5 & 5 & 5 & 5 & 6 \\
\hline Tap & 0 & 0 & 0 & 0 & 0 & 1 & 1 & 1 & 2 & 2 & 4 \\
\hline Shunt & 0 & 0 & 0 & 0 & 0 & 0 & 0 & 1 & 1 & 2 & 2 \\
\hline \multicolumn{12}{|c|}{ Resultado Final com o Algoritmo 7 usando a SENOIDAL } \\
\hline $\mathrm{N}$ & 0 & 0 & 0 & 4 & 4 & 6 & 6 & 7 & 8 & 9 & 12 \\
\hline Perdas $[\mathrm{MW}]$ & 17.54 & 17.54 & 17.54 & 16.31 & 16.31 & 16.08 & 16.08 & 16.04 & 16.01 & 15.98 & 15.98 \\
\hline$\Delta \mathrm{N}$ & - & 0 & 0 & 4 & 0 & 2 & 0 & 1 & 1 & 1 & 3 \\
\hline$\Delta \mathrm{P}[\mathrm{MW}]$ & - & 0 & 0 & -1.23 & 0 & -0.23 & 0 & -0.04 & -0.03 & -0.03 & 0 \\
\hline Iteração & 2 & 2 & 2 & 2 & 2 & 2 & 2 & 4 & 2 & 6 & 6 \\
\hline Tensão & 0 & 0 & 0 & 4 & 4 & 5 & 5 & 5 & 5 & 5 & 6 \\
\hline Tap & 0 & 0 & 0 & 0 & 0 & 1 & 1 & 1 & 2 & 2 & 4 \\
\hline Shunt & 0 & 0 & 0 & 0 & 0 & 0 & 0 & 1 & 1 & 2 & 2 \\
\hline Tempo (s) & 0.384 & 0.534 & 0.524 & 0.672 & 0.654 & 0.756 & 0.886 & 0.890 & 0.798 & 1.206 & 1.280 \\
\hline \multicolumn{12}{|c|}{ Resultado Final com o Algoritmo 7 usando a POLINOMIAL } \\
\hline $\mathrm{N}$ & 0 & 0 & 0 & $\overline{4}$ & 4 & 6 & 6 & 7 & 8 & 9 & 12 \\
\hline Perdas $[\mathrm{MW}]$ & 17.54 & 17.54 & 17.54 & 16.31 & 16.31 & 16.08 & 16.08 & 16.04 & 16.01 & 15.98 & 15.98 \\
\hline$\Delta \mathrm{N}$ & - & 0 & 0 & 4 & 0 & 2 & 0 & 1 & 1 & 1 & 3 \\
\hline$\Delta \mathrm{P}[\mathrm{MW}]$ & - & 0 & 0 & -1.23 & 0 & -0.23 & 0 & -0.04 & -0.03 & -0.03 & 0 \\
\hline Iteração & 2 & 2 & 2 & 2 & 2 & 7 & 7 & 7 & 7 & 7 & 7 \\
\hline Tensão & 0 & 0 & 0 & 4 & 4 & 5 & 5 & 5 & 5 & 5 & 6 \\
\hline Tap & 0 & 0 & 0 & 0 & 0 & 1 & 1 & 1 & 2 & 2 & 4 \\
\hline Shunt & 0 & 0 & 0 & 0 & 0 & 0 & 0 & 1 & 1 & 2 & 2 \\
\hline Tempo (s) & 0.568 & 0.536 & 0.515 & 0.653 & 0.809 & 1.485 & 1.525 & 1.511 & 1.262 & 1.414 & 1.451 \\
\hline \multicolumn{12}{|c|}{ Resultado Final com o Algoritmo 7 usando a MISTA } \\
\hline $\mathrm{N}$ & 0 & 0 & 0 & 4 & 4 & 6 & 6 & 7 & 8 & 9 & 12 \\
\hline Perdas $[\mathrm{MW}]$ & 17.54 & 17.54 & 17.54 & 16.31 & 16.31 & 16.08 & 16.08 & 16.04 & 16.01 & 15.98 & 15.98 \\
\hline$\Delta \mathrm{N}$ & - & 0 & 0 & 4 & 0 & 2 & 0 & 1 & 1 & 1 & 3 \\
\hline$\Delta \mathrm{P}[\mathrm{MW}]$ & - & 0 & 0 & -1.23 & 0 & -0.23 & 0 & -0.04 & -0.03 & -0.03 & 0 \\
\hline Iteração & 2 & 2 & 2 & 2 & 2 & 2 & 2 & 4 & 2 & 4 & 5 \\
\hline Tensão & 0 & 0 & 0 & 4 & 4 & 5 & 5 & 5 & 5 & 5 & 6 \\
\hline Tap & 0 & 0 & 0 & 0 & 0 & 1 & 1 & 1 & 2 & 2 & 4 \\
\hline Shunt & 0 & 0 & 0 & 0 & 0 & 0 & 0 & 1 & 1 & 2 & 2 \\
\hline Tempo (s) & 0.398 & 0.495 & 0.413 & 0.653 & 0.651 & 0.772 & 0.858 & 0.896 & 0.657 & 1.017 & 1.126 \\
\hline
\end{tabular}


Tabela 6.42 - Resultados do sistema IEEE de 57 barras para os diferentes valores de $\omega$.

\begin{tabular}{|c|c|c|c|c|c|c|c|c|c|c|c|}
\hline \multicolumn{12}{|c|}{ Resultado Parcial com o Algoritmo 6 usando a SIGMOIDAL } \\
\hline$\omega$ & 0.0 & 0.1 & 0.2 & 0.3 & 0.4 & 0.5 & 0.6 & 0.7 & 0.8 & 0.9 & 1.0 \\
\hline $\mathrm{N}$ & 1 & 1 & 1 & 8 & 8 & 8 & 8 & 14 & 16 & 19 & $\overline{24}$ \\
\hline Perdas $[\mathrm{MW}]$ & 26.02 & 25.90 & 25.90 & 21.74 & 21.74 & 21.74 & 21.74 & 21.09 & 20.99 & 20.96 & 20.92 \\
\hline$\Delta \mathrm{N}$ & - & 0 & 0 & 7 & 0 & 0 & 0 & 6 & 2 & 3 & 5 \\
\hline$\Delta \mathrm{P}[\mathrm{MW}]$ & - & -0.12 & 0 & -4.16 & 0 & 0 & 0 & -0.65 & -0.10 & -0.03 & -0.04 \\
\hline IterSIG & 6 & 6 & 6 & 5 & 5 & 5 & 5 & 5 & 5 & 5 & 5 \\
\hline Tensão & 1 & 1 & 1 & 7 & 7 & 7 & 7 & 7 & 7 & 7 & 7 \\
\hline Tap & 0 & 0 & 0 & 0 & 1 & 1 & 1 & 7 & 8 & 11 & 15 \\
\hline Shunt & 0 & 0 & 0 & 0 & 0 & 0 & 0 & 0 & 1 & 1 & 2 \\
\hline \multicolumn{12}{|c|}{ Resultado Final com o Algoritmo 7 usando a SENOIDAL } \\
\hline $\mathrm{N}$ & 1 & 1 & 1 & 8 & 8 & 8 & 8 & 14 & 16 & 19 & 23 \\
\hline Perdas $[\mathrm{MW}]$ & 26.02 & 25.90 & 25.90 & 21.74 & 21.74 & 21.74 & 21.74 & 21.11 & 21.01 & 20.99 & 20.95 \\
\hline$\Delta \mathrm{N}$ & - & 0 & 0 & 7 & 0 & 0 & 0 & 6 & 2 & 3 & 4 \\
\hline$\Delta \mathrm{P}[\mathrm{MW}]$ & - & -0.12 & 0 & -4.16 & 0 & 0 & 0 & -0.63 & -0.10 & -0.02 & -0.04 \\
\hline Iteração & 2 & 2 & 2 & 4 & 4 & 4 & 4 & 6 & 6 & 6 & 8 \\
\hline Tensão & 1 & 1 & 1 & 7 & 7 & 7 & 7 & 7 & 7 & 7 & 7 \\
\hline Tap & 0 & 0 & 0 & 0 & 1 & 1 & 1 & 7 & 8 & 11 & 15 \\
\hline Shunt & 0 & 0 & 0 & 0 & 0 & 0 & 0 & 0 & 1 & 1 & 1 \\
\hline Tempo (s) & 1.055 & 0.978 & 1.155 & 1.109 & 1.301 & 1.521 & 1.087 & 2.086 & 1.872 & 1.485 & 1.962 \\
\hline \multicolumn{12}{|c|}{ Resultado Final com o Algoritmo 7 usando a POLINOMIAL } \\
\hline $\mathrm{N}$ & 1 & 1 & 1 & 8 & 8 & 8 & 8 & 14 & 16 & 19 & 23 \\
\hline Perdas $[\mathrm{MW}]$ & 26.02 & 25.90 & 25.90 & 21.75 & 21.75 & 21.75 & 21.75 & 21.11 & 21.01 & 20.99 & 20.95 \\
\hline$\Delta \mathrm{N}$ & - & 0 & 0 & 7 & 0 & 0 & 0 & 6 & 2 & 3 & 4 \\
\hline$\Delta \mathrm{P}[\mathrm{MW}]$ & - & -0.12 & 0 & -4.15 & 0 & 0 & 0 & -0.63 & -0.10 & -0.02 & -0.04 \\
\hline Iteração & 2 & 2 & 2 & 5 & 5 & 5 & 5 & 7 & 7 & 7 & 7 \\
\hline Tensão & 1 & 1 & 1 & 7 & 7 & 7 & 7 & 7 & 7 & 7 & 7 \\
\hline Tap & 0 & 0 & 0 & 0 & 1 & 1 & 1 & 7 & 8 & 11 & 15 \\
\hline Shunt & 0 & 0 & 0 & 0 & 0 & 0 & 0 & 0 & 1 & 1 & 1 \\
\hline Tempo (s) & 1.096 & 1.231 & 1.026 & 1.403 & 1.561 & 1.691 & 1.688 & 1.097 & 1.482 & 1.348 & 2.147 \\
\hline \multicolumn{12}{|c|}{ Resultado Final com o Algoritmo 7 usando a MISTA } \\
\hline $\mathrm{N}$ & 1 & 1 & 1 & 8 & 8 & 8 & 8 & 14 & 16 & 19 & 23 \\
\hline Perdas $[\mathrm{MW}]$ & 26.02 & 25.90 & 25.90 & 21.74 & 21.74 & 21.74 & 21.74 & 21.11 & 21.01 & 20.99 & 20.95 \\
\hline$\Delta \mathrm{N}$ & - & 0 & 0 & 7 & 0 & 0 & 0 & 6 & 2 & 3 & 4 \\
\hline$\Delta \mathrm{P}[\mathrm{MW}]$ & - & -0.12 & 0 & -4.16 & 0 & 0 & 0 & -0.63 & -0.10 & -0.02 & -0.04 \\
\hline Iteração & 2 & 2 & 2 & 4 & 4 & 4 & 4 & 6 & 6 & 6 & 6 \\
\hline Tensão & 1 & 1 & 1 & 7 & 7 & 7 & 7 & 7 & 7 & 7 & 7 \\
\hline Tap & 0 & 0 & 0 & 0 & 1 & 1 & 1 & 7 & 8 & 11 & 15 \\
\hline Shunt & 0 & 0 & 0 & 0 & 0 & 0 & 0 & 0 & 1 & 1 & 1 \\
\hline Tempo (s) & 0.983 & 1.246 & 1.155 & 1.605 & 1.537 & 1.422 & 1.460 & 1.901 & 2.128 & 1.925 & 1.876 \\
\hline
\end{tabular}


Tabela 6.43 - Resultados do sistema IEEE de 118 barras para os diferentes valores de $\omega$.

\begin{tabular}{|c|c|c|c|c|c|c|c|c|c|c|c|c|c|c|c|c|}
\hline$\omega$ & 0.0 & 0.1 & 0.2 & 0.3 & 0.4 & 0.5 & 0.52 & 0.53 & 0.535 & 0.538 & 0.54 & 0.6 & 0.7 & 0.8 & 0.9 & 1.0 \\
\hline \multicolumn{17}{|c|}{ Resultado Parcial com o Algoritmo 6 usando a SIGMOIDAL } \\
\hline $\mathrm{N}$ & 3 & 3 & 3 & 6 & 10 & 19 & 25 & 27 & 28 & 39 & 57 & 59 & 59 & 60 & 61 & 67 \\
\hline Perdas [MW] & 132.47 & 132.47 & 132.47 & 130.79 & 127.18 & 124.09 & 122.46 & 121.99 & 121.74 & 117.70 & 109.70 & 106.39 & 106.36 & 106.25 & 106.20 & 106.07 \\
\hline$\Delta \mathrm{N}$ & - & 0 & 0 & 3 & 4 & 9 & 6 & 2 & 1 & 11 & 18 & 2 & 0 & 1 & 1 & 7 \\
\hline$\Delta \mathrm{P}[\mathrm{MW}]$ & - & 0 & 0 & -1.68 & -3.61 & -3.09 & -1.63 & -0.47 & -0.25 & -4.04 & -8 & -3.31 & -0.03 & -0.09 & -0.05 & -0.13 \\
\hline IterSIG & 6 & 6 & 6 & 5 & 5 & 5 & 4 & 4 & 4 & 4 & 4 & 3 & 3 & 3 & 3 & 4 \\
\hline Tensão & 2 & 2 & 2 & 5 & 9 & 17 & 23 & 25 & 26 & 31 & 50 & 54 & 54 & 54 & 54 & 54 \\
\hline Tap & 0 & 0 & 0 & 0 & 1 & 2 & 2 & 2 & 2 & 8 & 6 & 4 & 4 & 5 & 6 & 9 \\
\hline Shunt & 1 & 1 & 1 & 1 & 0 & 0 & 0 & 0 & 0 & 0 & 1 & 1 & 1 & 1 & 1 & 4 \\
\hline \multicolumn{17}{|c|}{ Resultado Final com o Algoritmo 7 usando a SENOIDAL } \\
\hline $\mathrm{N}$ & 3 & $\overline{3}$ & 3 & 6 & 10 & 19 & 25 & 27 & 28 & 39 & 56 & 59 & 59 & 60 & 61 & 67 \\
\hline Perdas $[\mathrm{MW}]$ & 132.47 & 132.47 & 132.47 & 130.79 & 127.18 & 124.09 & 122.47 & 121.99 & 121.74 & 117.71 & 109.71 & 106.40 & 106.37 & 106.28 & 106.23 & 106.11 \\
\hline$\Delta \mathrm{N}$ & - & 0 & 0 & 3 & 4 & 9 & 6 & 2 & 1 & 11 & 17 & 3 & 0 & 1 & 1 & 6 \\
\hline$\Delta \mathrm{P}[\mathrm{MW}]$ & - & 0 & 0 & -1.68 & -3.61 & -3.09 & -1.62 & -0.48 & -0.25 & -4.03 & -8 & -3.31 & -0.03 & -0.09 & -0.05 & -0.12 \\
\hline Iteração & 2 & 2 & 2 & 2 & 3 & 3 & 5 & 3 & 3 & 4 & 5 & 5 & 5 & 5 & 5 & 5 \\
\hline Tensão & 2 & 2 & 2 & 5 & 9 & 17 & 23 & 25 & 26 & 31 & 50 & 54 & 54 & 54 & 54 & 54 \\
\hline Tap & 0 & 0 & 0 & 0 & 1 & 2 & 2 & 2 & 2 & 8 & 6 & 4 & 4 & 5 & 6 & 9 \\
\hline Shunt & 1 & 1 & 1 & 1 & 0 & 0 & 0 & 0 & 0 & 0 & 0 & 1 & 1 & 1 & 1 & 4 \\
\hline Tempo $[\mathrm{s}]$ & 1.011 & 1.175 & 1.192 & 1.048 & 1.475 & 1.266 & 2.124 & 1.089 & 1.339 & 2.023 & 2.906 & 1.765 & 2.159 & 2.616 & 2.088 & 2.603 \\
\hline \multicolumn{17}{|c|}{ Resultado Final com o Algoritmo 7 usando a POLINOMIAL } \\
\hline $\mathrm{N}$ & 3 & 3 & 3 & 6 & 10 & 19 & 25 & 27 & 28 & 39 & 56 & 59 & 59 & 60 & 61 & 67 \\
\hline Perdas [MW] & 132.47 & 132.47 & 132.47 & 130.79 & 127.18 & 124.09 & 122.47 & 121.99 & 121.74 & 117.71 & 109.71 & 106.40 & 106.37 & 106.28 & 106.23 & 106.11 \\
\hline$\Delta \mathrm{N}$ & - & 0 & 0 & 3 & 4 & 9 & 6 & 2 & 1 & 11 & 17 & 3 & 0 & 1 & 1 & 6 \\
\hline$\Delta \mathrm{P}[\mathrm{MW}]$ & - & 0 & 0 & -1.68 & -3.61 & -3.09 & -1.62 & -0.48 & -0.25 & -4.03 & -8 & -3.31 & -0.03 & -0.09 & -0.05 & -0.12 \\
\hline Iteração & 1 & 2 & 2 & 2 & 4 & 8 & 7 & 4 & 4 & 6 & 6 & 6 & 5 & 7 & 7 & 7 \\
\hline Tensão & 2 & 2 & 2 & 5 & 9 & 17 & 23 & 25 & 26 & 31 & 50 & 54 & 54 & 54 & 54 & 54 \\
\hline Tap & 0 & 0 & 0 & 0 & 1 & 2 & 2 & 2 & 2 & 8 & 6 & 4 & 4 & 5 & 6 & 9 \\
\hline Shunt & 1 & 1 & - & 1 & 0 & 0 & 0 & 0 & 0 & 0 & 0 & 1 & 1 & 1 & 1 & 4 \\
\hline Tempo $[\mathrm{s}]$ & 0.908 & 1.146 & 0.924 & 1.044 & 1.646 & 2.485 & 1.960 & 1.286 & 1.644 & 3.702 & 3.598 & 1.966 & 2.088 & 2.756 & 2.217 & 3.452 \\
\hline \multicolumn{17}{|c|}{ Resultado Final com o Algoritmo 7 usando a MISTA } \\
\hline $\mathrm{N}$ & 3 & 3 & 3 & 0 & 10 & 19 & 25 & 27 & 28 & 39 & 56 & 59 & 59 & 60 & 61 & 66 \\
\hline Perdas [MW] & 132.47 & 132.47 & 132.47 & 130.79 & 127.18 & 124.09 & 122.47 & 121.99 & 121.74 & 117.71 & 109.72 & 106.40 & 106.37 & 106.28 & 106.24 & 106.20 \\
\hline$\Delta \mathrm{N}$ & - & 0 & 0 & 3 & 4 & 9 & 6 & 2 & 1 & 11 & 17 & 3 & 0 & 1 & 1 & 5 \\
\hline$\Delta \mathrm{P}[\mathrm{MW}]$ & - & 0 & 0 & -1.68 & -3.61 & -3.09 & -1.62 & -0.48 & -0.25 & -4.03 & -7.99 & -3.32 & -0.03 & -0.09 & -0.04 & -0.04 \\
\hline Iteração & 2 & 2 & 2 & 2 & 3 & 3 & 5 & 3 & 3 & 4 & 4 & 4 & 4 & 5 & 5 & 5 \\
\hline Tensão & 2 & 2 & 2 & 5 & 9 & 17 & 23 & 25 & 26 & 31 & 50 & 54 & 54 & 54 & 54 & 54 \\
\hline Tap & 0 & 0 & 0 & 0 & 1 & 2 & 2 & 2 & 2 & 8 & 6 & 4 & 4 & 5 & 6 & 8 \\
\hline Shunt & 1 & 1 & 1 & 1 & 0 & 0 & 0 & 0 & 0 & 0 & 0 & 1 & 1 & 1 & 1 & 4 \\
\hline Tempo $[\mathrm{s}]$ & 1.128 & 1.217 & 1.115 & 1.100 & 1.413 & 1.269 & 1.363 & 1.170 & 1.191 & 2.218 & 1.695 & 1.380 & 2.205 & 1.960 & 2.014 & 2.701 \\
\hline
\end{tabular}


Tabela 6.44 - Resultados do sistema IEEE de 300 barras para os diferentes valores de $\omega$.

\begin{tabular}{|c|c|c|c|c|c|c|c|c|c|c|c|}
\hline \multicolumn{12}{|c|}{ Resultado Parcial com o Algoritmo 6 usando a SIGMOIDAL } \\
\hline$\omega$ & 0.0 & 0.1 & 0.2 & 0.3 & 0.4 & 0.5 & 0.6 & 0.7 & 0.8 & 0.9 & 1.0 \\
\hline $\mathrm{N}$ & 2 & 1 & 1 & 16 & 30 & 48 & 60 & 82 & 88 & 102 & 172 \\
\hline Perdas $[\mathrm{MW}]$ & 404.97 & 404.97 & 404.97 & 382.65 & 370.84 & 356.37 & 351.35 & 344.45 & 342.83 & 341.58 & 340.85 \\
\hline$\Delta \mathrm{N}$ & - & -1 & 0 & 15 & 14 & 18 & 12 & 22 & 6 & 14 & 70 \\
\hline$\Delta \mathrm{P}[\mathrm{MW}]$ & - & 0 & 0 & -22.32 & -11.81 & -14.47 & -5.02 & -6.90 & -1.62 & -1.25 & -0.73 \\
\hline IterSIG & 2 & 2 & 2 & 3 & 3 & 3 & 3 & 3 & 3 & 3 & 3 \\
\hline Tensão & 0 & 0 & 0 & 9 & 15 & 24 & 25 & 40 & 43 & 45 & 62 \\
\hline Tap & 0 & 0 & 0 & 6 & 14 & 23 & 33 & 40 & 43 & 55 & 103 \\
\hline Shunt & 2 & 1 & 1 & 1 & 1 & 1 & 2 & 2 & 2 & 2 & 7 \\
\hline \multicolumn{12}{|c|}{ Resultado Final com o Algoritmo 7 usando a SENOIDAL } \\
\hline $\mathrm{N}$ & 2 & $\overline{2}$ & 2 & 16 & 35 & 53 & 70 & 88 & 99 & 114 & 173 \\
\hline Perdas $[\mathrm{MW}]$ & 405.25 & 405.25 & 405.25 & 384.27 & 371.92 & 357.71 & 352.77 & 346.05 & 344.29 & 342.97 & 342.28 \\
\hline$\Delta \mathrm{N}$ & - & 0 & 0 & 14 & 19 & 18 & 17 & 18 & 11 & 15 & 59 \\
\hline$\Delta \mathrm{P}[\mathrm{MW}]$ & - & 0 & 0 & -20.98 & -12.35 & -14.21 & -4.94 & -6.72 & -1.76 & -1.32 & -0.69 \\
\hline Iteração & 10 & 10 & 9 & 9 & 10 & 10 & 10 & 10 & 10 & 10 & 10 \\
\hline Tensão & 1 & 1 & 1 & 10 & 20 & 29 & 35 & 46 & 54 & 57 & 69 \\
\hline Tap & 0 & 0 & 0 & 5 & 14 & 23 & 33 & 40 & 43 & 55 & 97 \\
\hline Shunt & 1 & 1 & 1 & 1 & 1 & 1 & 2 & 2 & 2 & 2 & 7 \\
\hline Tempo (s) & 3.774 & 3.775 & 3.756 & 4.354 & 6.073 & 8.470 & 13.137 & 18.239 & 21.410 & 17.599 & 22.143 \\
\hline \multicolumn{12}{|c|}{ Resultado Final com o Algoritmo 7 usando a POLINOMIAL } \\
\hline $\mathrm{N}$ & 4 & 4 & 4 & 18 & 36 & 53 & 71 & 88 & 98 & 114 & 174 \\
\hline Perdas $[\mathrm{MW}]$ & 404.98 & 404.98 & 404.98 & 383.79 & 372.95 & 357.61 & 352.59 & 345.60 & 344.16 & 342.74 & 342.07 \\
\hline$\Delta \mathrm{N}$ & - & 0 & 0 & 14 & 18 & 17 & 18 & 17 & 10 & 16 & 60 \\
\hline$\Delta \mathrm{P}[\mathrm{MW}]$ & - & 0 & 0 & -21.19 & -10.84 & -15.34 & -5.02 & -6.99 & -1.44 & -1.42 & -0.67 \\
\hline Iteração & 7 & 7 & 9 & 9 & 9 & 9 & 9 & 9 & 9 & 9 & 9 \\
\hline Tensão & 2 & 2 & 2 & 11 & 20 & 29 & 36 & 46 & 53 & 57 & 69 \\
\hline Tap & 1 & 1 & 1 & 6 & 15 & 23 & 33 & 40 & 43 & 55 & 98 \\
\hline Shunt & 1 & 1 & 1 & 1 & 1 & 1 & 2 & 2 & 2 & 2 & 7 \\
\hline Tempo (s) & 3.555 & 3.878 & 5.179 & 5.412 & 6.310 & 7.988 & 8.562 & 13.009 & 15.262 & 14.471 & 20.974 \\
\hline \multicolumn{12}{|c|}{ Resultado Final com o Algoritmo 7 usando a MISTA } \\
\hline $\mathrm{N}$ & $\overline{4}$ & 4 & 2 & 18 & 38 & 53 & 70 & 88 & 97 & 113 & 172 \\
\hline Perdas $[\mathrm{MW}]$ & 404.98 & 404.98 & 405.25 & 383.83 & 371.01 & 357.61 & 352.66 & 345.41 & 343.68 & 342.55 & 341.74 \\
\hline$\Delta \mathrm{N}$ & - & 0 & -2 & 16 & 20 & 15 & 17 & 18 & 9 & 16 & 59 \\
\hline$\Delta \mathrm{P}[\mathrm{MW}]$ & - & 0 & 0.27 & -21.42 & -12.82 & -13.40 & -4.95 & -7.25 & -1.73 & -1.13 & -0.81 \\
\hline Iteração & 9 & 9 & 6 & 9 & 10 & 9 & 9 & 9 & 9 & 9 & 9 \\
\hline Tensão & 2 & 2 & 0 & 11 & 22 & 29 & 35 & 46 & 52 & 56 & 69 \\
\hline Tap & 1 & 1 & 1 & 6 & 15 & 23 & 33 & 40 & 43 & 55 & 96 \\
\hline Shunt & 1 & 1 & 1 & 1 & 1 & 1 & 2 & 2 & 2 & 2 & 7 \\
\hline Tempo (s) & 3.682 & 3.653 & 3.154 & 4.555 & 6.271 & 7.532 & 10.952 & 12.488 & 13.573 & 14.079 & 18.624 \\
\hline
\end{tabular}

As Figuras 6.46, 6.47, 6.48, 6.49 e 6.50 apresentam as perdas ativas pelo número de ações de controle para os sistemas IEEE de 14, 30, 57, 118 e 300 barras, respectivamente, usando o Algoritmo 7 para cada função de discretização. O resultado parcial encontrado pelo Algoritmo 6 é nomeado como SIGMOIDAL (Parcial). O resultado final encontrado pelo Algoritmo 7 é nomeado apenas pela função de discretização que foi utilizada. 


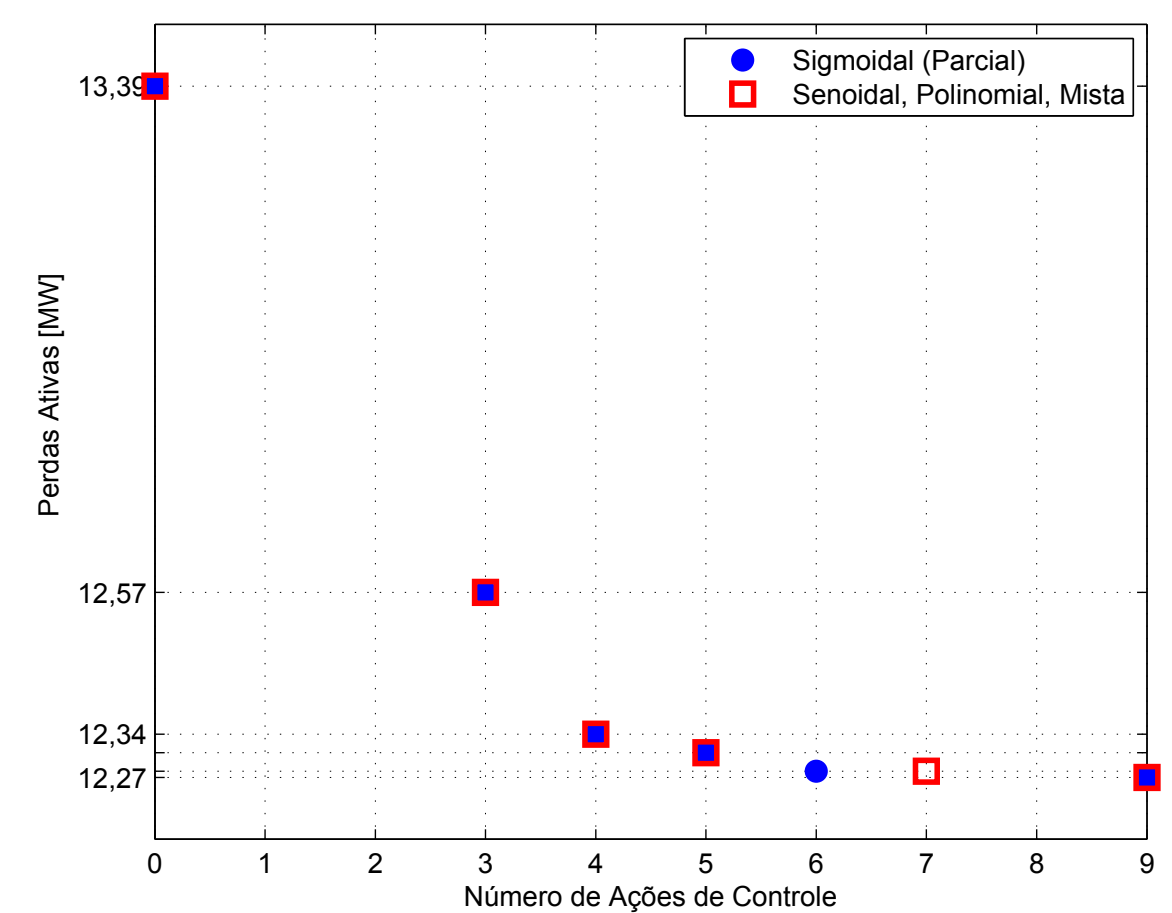

Figura 6.46 - Perdas ativas pelo número de ações de controle para o sistema IEEE de 14 barras.

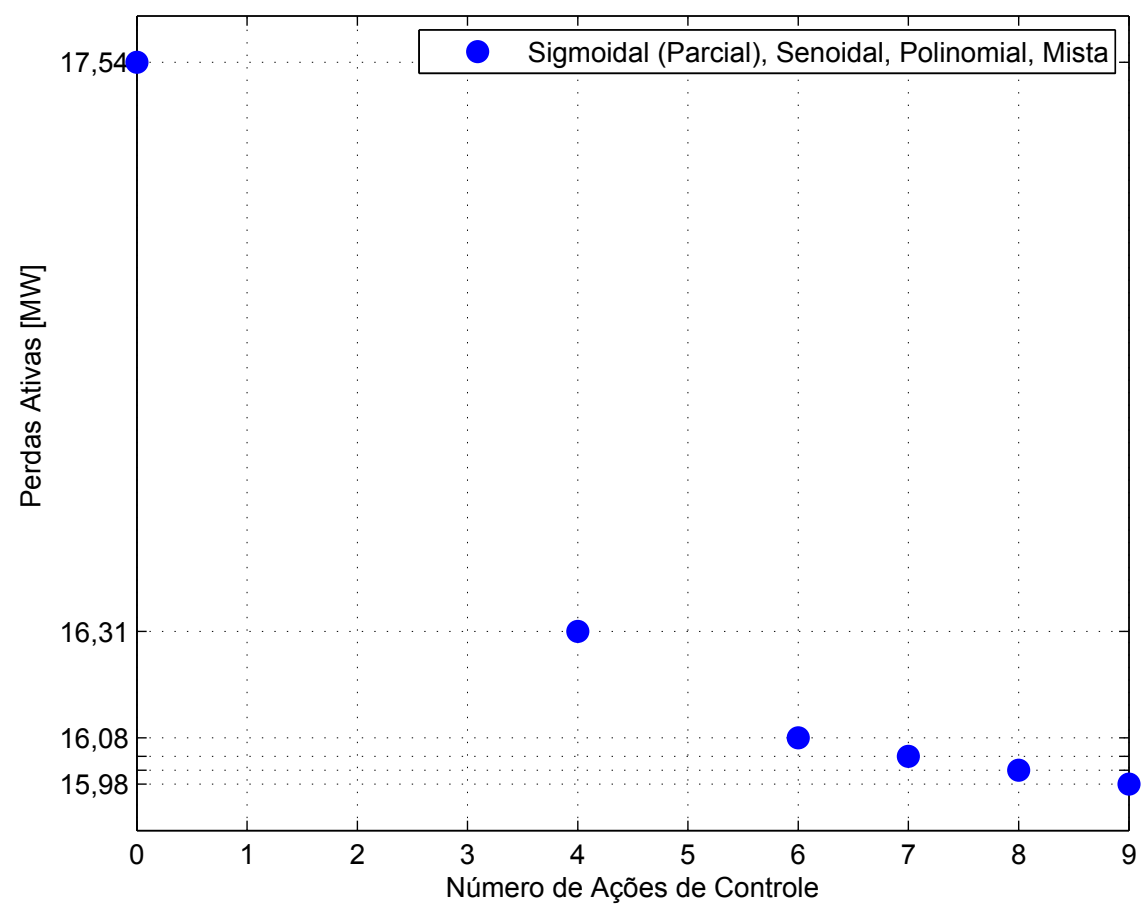

Figura 6.47 - Perdas ativas pelo número de ações de controle para o sistema IEEE de 30 barras. 


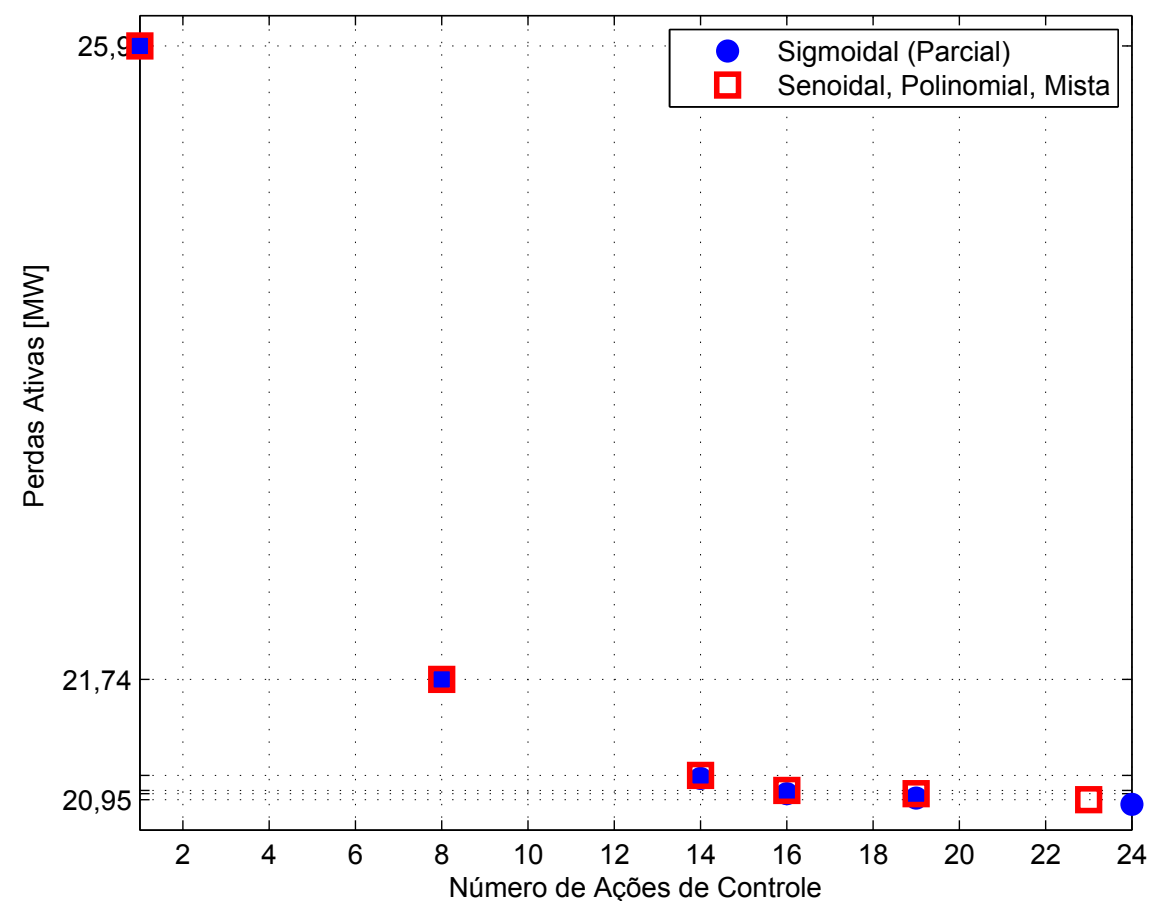

Figura 6.48 - Perdas ativas pelo número de ações de controle para o sistema IEEE de 57 barras.

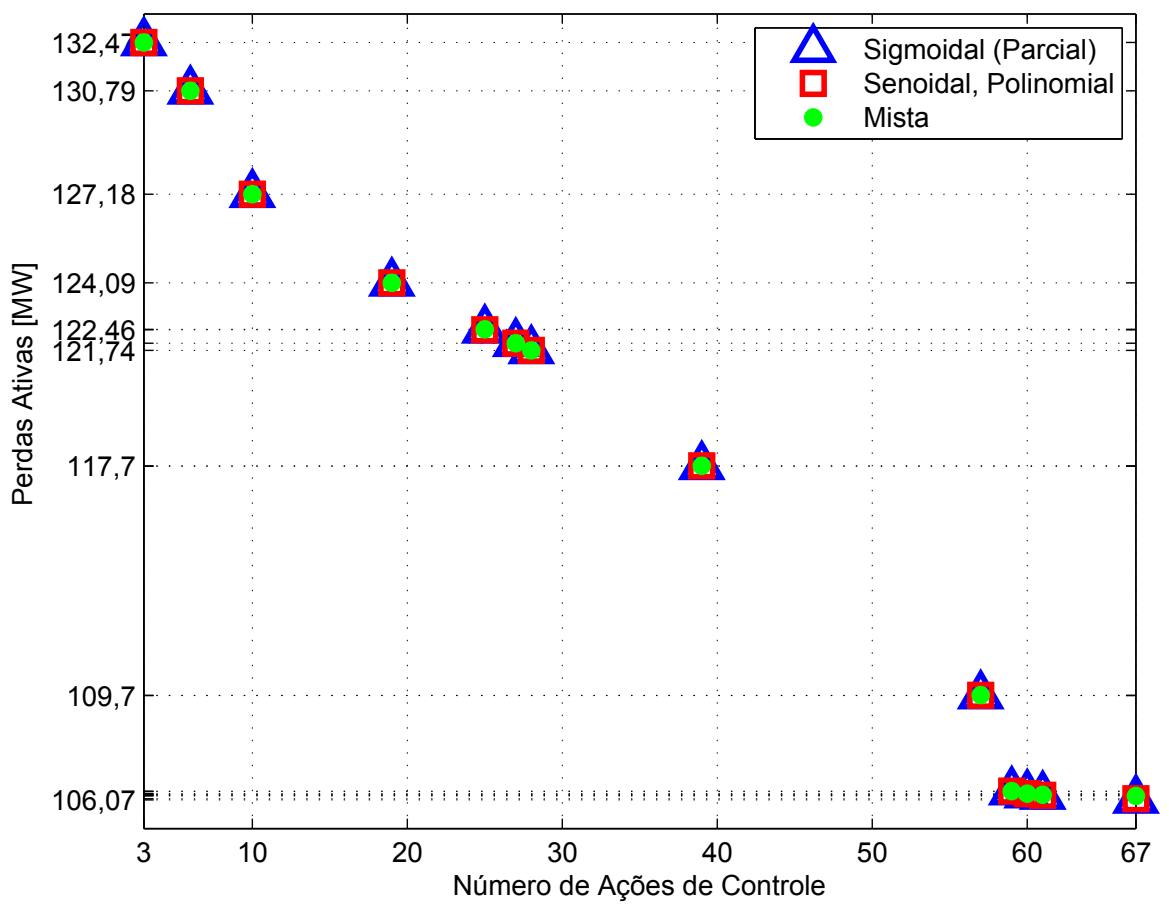

Figura 6.49 - Perdas ativas pelo número de ações de controle para o sistema IEEE de 118 barras. 


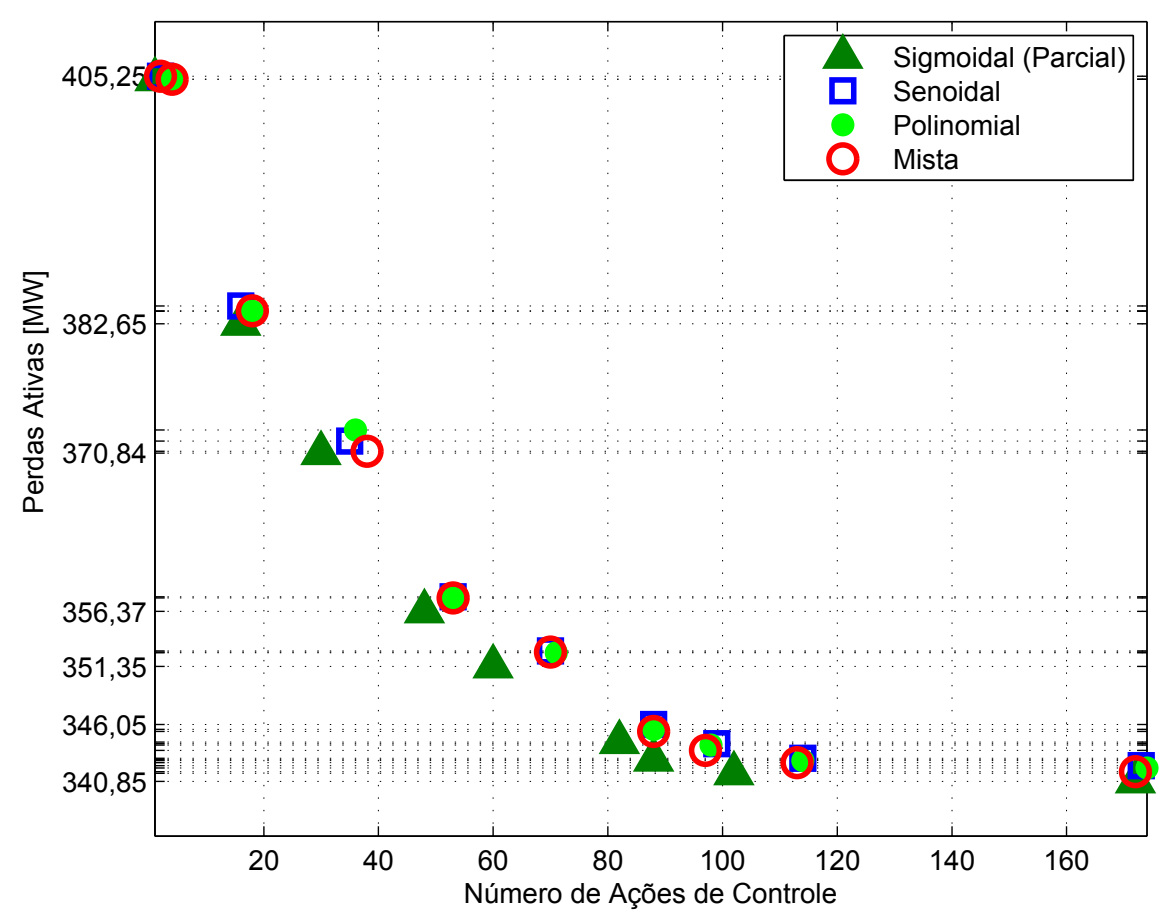

Figura 6.50 - Perdas ativas pelo número de ações de controle para o sistema IEEE de 300 barras.

\subsubsection{Análise dos Resultados}

Para o sistema de 14 barras, todas as funções de discretização chegam ao mesmo resultado final, porém para o peso 0.9 o resultado parcial possui seis ações de controles enquanto o resultado final possui sete ações de controle. Isso acontece pois o resultado parcial é contínuo e ao discretizar o tap de transformador e o shunt de barra, alguns ajustes devem ser feitos para que o resultado continue factível, de modo que mais uma tensão é ajustada.

Para o sistema de 30 barras, todas as funções de discretização chegam ao mesmo ponto ótimo local e o resultado parcial coincide com o resultado final.

Para o sistema de 57 barras, todas as funções de discretização chegam ao mesmo resultado final. Existe uma diferença mínima para os pesos $0.7,0.8$ e 0.9 entre o resultado final e o parcial. Já para $\omega=1.0$ o resultado final possui um shunt de barra ajustado a mais que o resultado parcial.

Para o sistema de 118 barras, as funções de discretização senoidal e polinomial chegam ao mesmo resultado final. Para três pesos as funções senoidal e polinomial são melhores que a função de discretização mista.

Para o sistema de 300 barras, fica nítido que o resultado parcial é um limitante inferior para o resultado final com as funções de discretização. As funções de discretização senoidal e polinomial encontraram nove soluções não dominadas enquanto a função de discretização mista 
encontrou dez soluções não dominadas. Comparando ponto a ponto e removendo as soluções dominadas obtemos a Figura 6.51. Note que a função senoidal possui três soluções na curva combinada, a polinomial possui quatro e a função de discretização mista possui nove soluções.

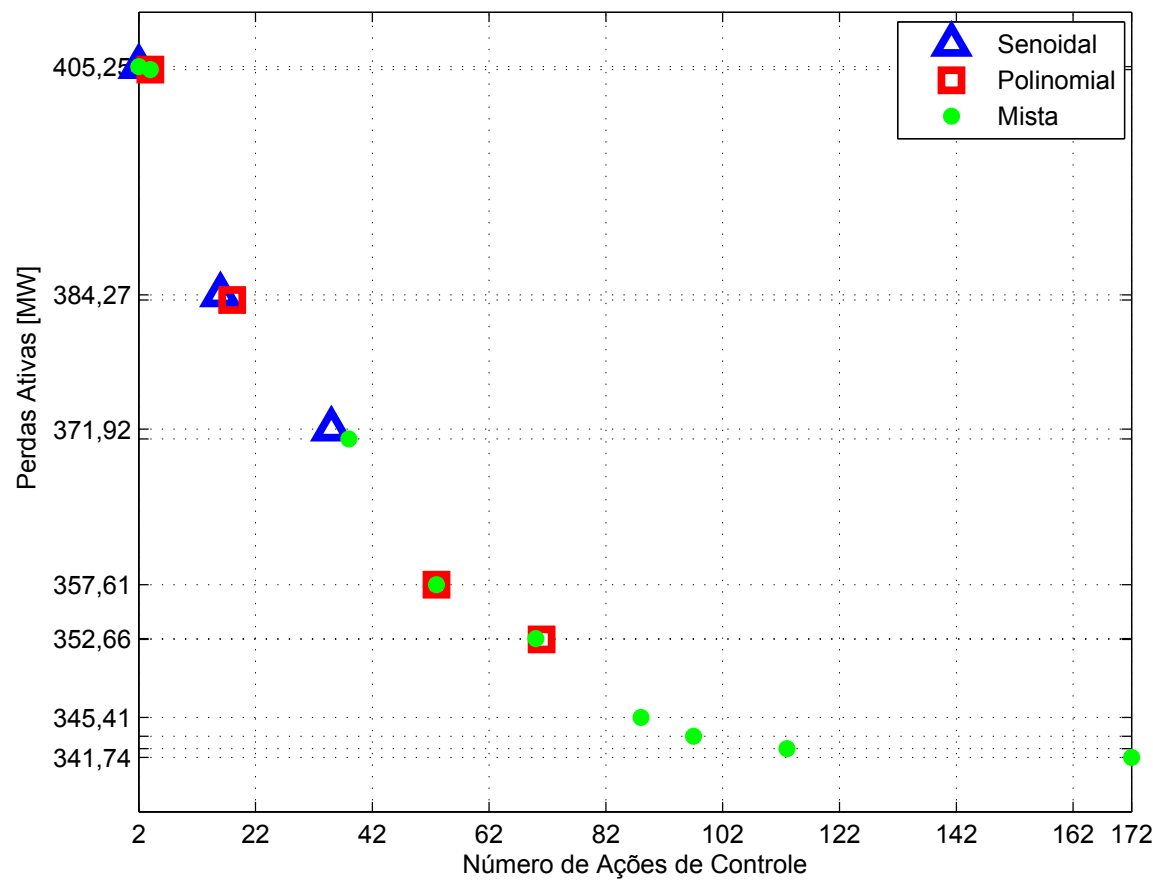

Figura 6.51 - Perdas ativas pelo número de ações de controle para o sistema de 300 barras. Soluções não dominadas geradas pela combinação das soluções das funções de discretização.

\subsubsection{Conclusões}

Apresentamos aqui resultados para o problema de FPO multiobjetivo com taps de transformadores e bancos de capacitores e reatores shunts discretos, tendo por objetivo verificar a eficácia da estratégia multiestágio apresentada no Algoritmo 7 para o tratamento do problema multiobjetivo com variáveis discretas. O problema de FPO multiobjetivo com taps de transformadores e bancos de capacitores e reatores shunts discretos é modificado pelo método da soma ponderada deixando de ser um problema multiobjetivo. As variáveis binárias são tratadas pela função sigmoidal enquanto as variáveis discretas são tratadas por funções de discretização. Um algoritmo multiestágio para a resolução deste problema é apresentado, onde num primeiro momento o problema de FPO multiobjetivo, modificado pelo método da soma ponderada é resolvido considerando todas as variáveis contínuas, e num segundo momento, as variáveis são discretizadas. Testes numéricos comprovam que a função Sigmoidal para o tratamento das variáveis binárias apresentam um ótimo desempenho combinada com as funções de discretização chegando a uma solução factível para o problema em questão. Por fim, na próxima subseção, apresentamos as conclusões gerais sobre os resultados para o problema de FPO multiobjetivo. 


\subsubsection{Conclusões Gerais}

Nesta seção, apresentamos resultados para o problema de FPO multiobjetivo com: 1) taps de transformadores contínuos e bancos de capacitores e reatores shunts fixos; 2) taps de transformadores discretos e elementos shunts fixos; e 3) taps de transformadores e elementos shunts discretos. Os objetivos desta seção eram verificar a eficácia das estratégias para o tratamento multiobjetivo, com variáveis contínuas e discretas, e o tratamento das variáveis binárias relacionadas as ações de controles. Para modelar o problema multiobjetivo, utilizou-se dois métodos que modificam o problema multiobjetivo em um problema mono-objetivo: Soma Ponderada e $\epsilon$-Restrito. Para o tratamento das variáveis discretas utiliza-se as funções de discretização Senoidal, Polinomial e Mista. Já para o tratamento das variáveis binárias relacionadas às ações de controles utilizou-se uma função Quadrática e uma função Sigmoidal. Os resultados comprovam que tanto o método da soma ponderada quanto o método $\epsilon$-restrito, combinados com as funções para o tratamento das variáveis binárias, são eficientes para encontrar soluções factíveis para o problema de FPO multiobjetivo com taps de transformadores contínuos e bancos de capacitores e reatores shunts fixos. Entretanto, ao comparar as duas estratégias o método da soma ponderada com a função sigmoidal se sobressai. Para o problema de FPO multiobjetivo com taps de transformadores discretos e bancos de capacitores e reatores shunts fixos, a modelagem do problema multiobjetivo se deu pelo método $\epsilon$-restrito juntamente com a função quadrática para o tratamento das variáveis binárias. O método se mostra eficiente para as funções de discretização senoidal e polinomial, tendo um melhor desempenho com a polinomial. Já para o problema de FPO multiobjetivo com taps de transformadores e bancos de capacitores e reatores shunts discretos, utilizou-se o método da soma ponderada com a função sigmoidal para a modelagem das variáveis binárias, e as variáveis discretas foram tratadas pelas estratégias de discretização, senoidal, polinomial e mista. O método da soma ponderada com a função sigmoidal é eficiente em encontrar uma solução factível para o problema e possui um melhor desempenho em conjunto com a função de discretização mista. Para a resolução dos modelos de FPO multiobjetivo modificados foram utilizados os solvers CONOPT e IPOPT, e para comparação entre as estratégias desenvolvidas para o tratamento das variáveis binárias os solvers DICOPT e KNITRO também foram utilizados. Conclui-se com estes que, as estratégias de modelagem desenvolvidas para as variáveis binárias do problema de FPO multiobjetivo combinadas com os solvers CONOPT e IPOPT são mais eficientes que os solvers DICOPT e KNITRO para os sistemas do IEEE. 


\section{Capítulo 7}

\section{Conclusões}

O problema de FPO é utilizado para analisar a operação dos SEPs, sendo um problema de otimização restrita, não linear, não convexo, estático, de grande porte, com variáveis contínuas e discretas, de difícil solução e de difícil aplicação real (on line) devido à presença de uma grande quantidade de variáveis discretas. Um dos objetivos de um FPO é a determinação dos ajustes das variáveis de controle de um SEE responsáveis por otimizar um determinado desempenho operacional do sistema.

Quando se realiza a representação matemática de um problema prático, normalmente algumas questões são omitidas, sejam porque determinados detalhes não possuem influência significativa na melhoria da solução ou porque o problema torna-se demasiadamente complexo para as metodologias de resolução existentes. Normalmente as restrições que devem ser consideradas são restrições operacionais que podem representar tanto limitações físicas de operação, como também limitações de recursos. Diante disto, aproximações são realizadas para facilitar a resolução do problema matemático, podendo levar a soluções distantes do que se espera do ideal, obrigando em algumas situações adaptar as soluções de forma que a solução seja factível ao problema original não relaxado.

A representação matemática de um problema de FPO está longe de conter todas as informações necessárias com relação ao problema real, e a inclusão da representação das variáveis discretas ao problema dificulta ainda mais sua solução. Além destes problemas, quando se visa minimizar as perdas ativas na transmissão, existe a necessidade da atuação da maioria dos controles para se obter uma solução ótima.

Neste contexto, este trabalho propôs a investigação e o desenvolvimento de estratégias para modelagem e resolução de problemas de FPO, onde existe a necessidade de se considerar as variáveis de controle associadas aos taps de transformadores em-fase e chaveamentos de bancos de capacitores e reatores shunt como variáveis discretas e existe a necessidade da limitação, e/ou até mesmo a minimização do número de ações de controle.

Num primeiro momento o problema de FPO foi modelado como um problema de PNLCD para a minimização das perdas ativas. As variáveis de controle associadas aos taps de transformadores e aos elementos shunt são modeladas como discretas, onde os taps de 
transformadores são ajustadas por passos iguais e os elementos shunt são ajustadas por passos diferentes. Para o tratamento dessas variáveis discretas propusemos três funções de discretização.

Para avaliar a eficácia das estratégias utilizou-se o solver CONOPT juntamente com a interface para modelagens de problemas de programação matemática GAMS e três cenários de carregamento. Os testes realizados com os sistemas elétricos do IEEE de 14, 30, 57, 118 e 300 barras indicam que os algoritmos propostos são eficientes e adequados para solução discreta de problemas de FPO com variáveis discretas igualmente espaçadas e não igualmente espaçadas. As comparações entre as funções de discretização mostram que o desempenho da estratégia mista que pode fornecer boas soluções e tem potencial para superar as outras estratégias devido à sua natureza sob medida para modelar diferentes variáveis discretas.

Num segundo momento, incluiu-se ao problema de FPO uma limitação no número de ações de controles. Os taps de transformadores são considerados discretos e tratados pela função polinomial de discretização e os bancos de capacitores e reatores shunts são considerados fixos. As variáveis binárias associadas as ações de controle são modeladas por uma função quadrática. Utilizou-se o solver CONOPT juntamente com a interface GAMS e os sistemas elétricos IEEE de 14, 30, 57, 118 e 300 barras para verificar a eficiência da estratégia e a modelagem das variáveis binárias. Os testes numéricos realizados nos sistemas-teste do IEEE têm mostrado bons resultados e eficácia em lidar com variáveis discretas e binários. O trade-off entre a realização ideal da função de objetivo, satisfazendo as restrições operacionais e reduzir o número de ajustes de controle para esses testes resultaram em boas soluções.

Num terceiro momento, o problema de FPO foi modelado como um problema de Otimização Multiobjetivo com objetivos de minimizar as perdas ativas na transmissão e minimizar as ações de controle com taps de transformadores contínuos e bancos de capacitores e reatores shunts fixos, taps de transformadores discretos e elementos shunts fixos, e para o caso onde ambos são considerados discretos. Para modelar o problema multiobjetivo, utilizou-se dois métodos que modificam o problema multiobjetivo em um problema mono-objetivo: Soma Ponderada e $\epsilon$-Restrito. Para o tratamento das variáveis discretas utiliza-se as funções de discretização Senoidal, Polinomial e Mista. Já para o tratamento das variáveis binárias relacionadas ao número de ações de controles utilizou-se uma função Quadrática e uma função Sigmoidal. Utilizou-se os solvers CONOPT e IPOPT e para comparação entre as estratégias desenvolvidas utilizou-se também os solvers DICOPT e KNITRO. Os testes realizados com os sistemas elétricos IEEE de 14, 30, 57, 118 e 300 barras indicam que com o aumento das variáveis de controle e da complexidade do problema, os solvers utilizados DICOPT e KNITRO se tornam inadequados. Conclui-se com estes que as estratégias de modelagem desenvolvidas para as variáveis binárias do problema de FPO multiobjetivo, combinadas com os solvers CONOPT e IPOPT são mais eficientes para os sistemas do IEEE.

Neste, utilizamos ferramentas de modelagem matemática a fim de modificar o problema de FPO, que consiste em um problema de PNLCD, e o problema de FPO multiobjetivo, aqui proposto, que contém variáveis binárias, em problemas de PNL, os quais podemos aplicar métodos clássicos como Gradiente Reduzido Generalizado. Funções de discretização foram utilizadas para 
o tratamento de variáveis discretas e uma nova metodologia utilizando uma função sigmoidal foi proposta para o tratamento de variáveis binárias.

No aspecto matemático, este trabalho contribuiu com uma nova estratégia para o tratamento de variáveis discretas e com duas novas estratégias para o tratamento de variáveis binárias. Do ponto de vista operacional foram desenvolvidos modelos para o problema de FPO levando em consideração o número elevado de ações de controles necessários para minimizar um certo desempenho do sistema. Em ambos os casos, estes conceitos podem ser generalizados e estendidos tendo o potencial para resolver problemas de PNL e de otimização multiobjetivo para diversas áreas da engenharia.

\subsection{Trabalhos Futuros}

As propostas de continuidade deste trabalho são as seguintes:

- Modificação do modelo de FPO com coordenadas polares para coordenadas retangulares.

O problema de FPO com minimização das perdas de potência ativa pode ser formulado como um problema de PNL utilizando equações e tensões em coordenadas retangulares como o proposto por (TORRES; QUINTANA, 1998). Essa metodologia pode ser vantajosa pois a Hessiana é constante e a expansão de Taylor é exata para o termo de ordem dois (OLIVEIRA; PROBST, 2010), possibilitando o uso de métodos de pontos interiores.

Além desses, seria possível a utilização do solver SNOPT (Sparse Nonlinear OPTimizer) que é um pacote para a resolução de problemas de otimização não-linear de grande porte e foi escrito por Philip Gill, Walter Murray e Michael Saunders. Ele emprega um algoritmo esparso de PQS com aproximações da matriz Hessiana.

- Estudo de técnicas de avaliação da qualidade da curva aproximada em relação a curva ótima de Pareto encontrada para um problema multiobjetivo.

Podemos avaliar de forma simples a qualidade de uma solução aproximada encontrada para um problema de otimização mono-objetivo, apenas fazendo a diferença relativa entre o valor aproximado e a solução ótima. Esta avaliação não pode ser feita de forma trivial em um problema de otimização multiobjetivo. Não existe uma forma simples e natural de medir a qualidade de um conjunto aproximado de soluções em relação ao conjunto de Pareto Ótimo.

Algumas técnicas de avaliação da qualidade da solução encontrada por heurísticas para problemas multiobjetivos foram propostas ao longo dos anos na literatura, como por exemplo, a avaliação analítica de Daniels (DANIELS, 1992). Daniels apresenta um algoritmo polinomial para determinar a qualidade da aproximação de um conjunto de pontos heurísticos em relação ao conjunto dos pontos Pareto-ótimos.

- Aplicação de Metaheurísticas em conjunto com os métodos clássicos para a resolução do problema multiobjetivo. 
Diferentes métodos têm sido propostos para resolver problemas de otimização multiobjetivo em sistemas de energia. A maioria dos métodos clássicos, como a soma ponderada e o $\epsilon$-restrito, necessitam de múltiplas avaliações e falham em encontrar todas as soluções ótimas de Pareto (STEPhen; SOMASUndARAM, 2012). Buscando contornar os problemas relacionados aos métodos clássicos de otimização multiobjetivo muitos esforços na literatura estão sendo demandados para o estudo das metaheurísticas. Algoritmo Genético (JEYANTHY; DEVARAJ, 2010), Enxame de Partículas (VARADARAJAN; SWARUP, 2008), Algoritmo de Busca Gravitacional (NIKNAM et al., 2013), PAES (Pareto Archived Evolution Strategy) (MONTOYA et al., 2010), são algumas das metaheurísticas usadas para resolver problemas de FPO multiobjetivo. A desvantagem das metaheurísticas está no alto tempo computacional demandado para encontrar soluções ótimas locais.

- Mudar a modelagem dos problemas de FPO apresentados para a modelagem de Programação Semi-definida.

A aplicação da Programação Semi-definida (PSD) para o problema de FPO atraiu recentemente interesse de pesquisa significativo. O problema de FPO é reformulado como um problema de PSD, modificando-o em um problema convexo (BAI et al., 2008), e aplicações recentes de uma relaxação de PSD oferece um método com tempo polinomial para calcular um ótimo global para uma grande subclasse de problemas de FPO, em particular (MOLZAHN; LESIEUTRE; DEMARCO, 2014) propuseram um teste para garantir a otimização global de uma solução candidata.

O problema de Despacho Econômico multiobjetivo é modificado pelo método da soma ponderada e o problema modificado resultante é remodelado como um problema convexo pela relaxação PSD em (JUBRIL; KOMOLAFE; ALAWODE, 2013), os resultado mostram que esta aplicação tem uma boa propriedade de convergência.

- Sequência Ótima de Ações de Controles.

Mais importante do que determinar um menor número de ações de controles em um problema de FPO é encontrar a sequência ótima de atuação destes controles. Capitanescu e Wehenkel (2011) propuseram uma estratégia para encontrar uma sequência de atuação para os controles, baseados na formulação do problema de FPO com limitação do número de ações de controles. Inicialmente o problema de FPO é resolvido permitindo-se a atuação de apenas um controle, na sequência permite-se a atuação de dois controles, posteriormente três controles e assim por diante. Essa formulação pode ser adaptada de modo a congelar os controles que atuaram em cada etapa do procedimento. 


\section{Referências Bibliográficas}

ABADIE, J.; CARPENTIER, J. Generalization of the wolfe reduced gradient method to the case of nonlinear constraints. Optimization, p. 37-47, 1969.

ABIDI, M. M.; POLYAK, R. A.; GRIVA, I. A.; MILI, L.; AMMARI, S. Optimal transformer tap selection using modified barrie-augmented lagrangian method. IEEE Transactions on Power Systems, v. 18, p. 251-257, 2003.

ALRASHIDI, M. R.; EL-HAWARY, M. E. Hybrid particle swarm optimization approach for solving the discrete opf problem considering the valve loading effects. IEEE Transactions on Power Systems, v. 22, p. 2030-2038, 2007.

BAI, X.; WEI, H.; FUJISAWA, K.; WANG, Y. Semidefinite programming for optimal power flow problems. Electrical Power and Energy Systems, p. 383-392, 2008.

BAKIRTZIS, A. G.; BISKAS, P. N.; ZOUMAS, C. E.; PETRIDIS, V. Optimal power flow by enhanced genetic algorithm. IEEE Transactions on Power Systems, v. 17, n. 2, p. 229-236, May 2002.

BAPtistA, E. C.; Belati, E. A.; SOUSA, V. A. de; COSTA, G. R. M. da. Primal-dual logarithmic barrier and augmented lagrangian function to the loss minimization in power systems. Electric Power Components and Systems, v. 34, n. 7, p. 775-784, 2006.

BURCHETT, R.; HAPP, H.; WIRGAU, K. Large scale optimal power flow. Power Apparatus and Systems, IEEE Transactions on, PAS-101, n. 10, p. 3722-3732, Oct 1982.

CAPITANESCU, F.; RAMOS, J. M.; PANCIATICI, P.; KIRSCHEN, D.; MARCOLINI, A. M.; PLATBROOD, L.; WEHENKEL, L. State-of-the-art, challenges, and future trends in security constrained optimal power flow. Electric Power Systems Research, v. 81, n. 8, p. 1731-1741, 2011.

CAPITANESCU, F.; ROSEHART, W.; WEHENKEL, L. Optimal power flow computations with constraints limiting the number of control actions. In: Power Tech Conference, Bucharest, Romania. [S.l.: s.n.], 2009.

CAPITANESCU, F.; WEHENKEL, L. Sensitivity-based approaches for handling discrete variables in optimal power flow computations. IEEE Transactions on Power Systems, v. 25, n. 4 , p. $1780-1789$, Nov. 2010. 
CAPITANESCU, F.; WEHENKEL, L. Redispatching active and reactive powers using a limited number of control actions. IEEE Transactions Power Systems, v. 26, p. 1221-1230, 2011.

CARPENTIER, J. L. Contribution à l'étude du dispatching économique. Bulletin de la Société Française des Electriciens, v. 3, n. 8, p. 431-447, 1962.

COSTA, G. Modified newton method for reactive dispatching. Electric Power and Energy Systems, v. 24, p. 431-447, 2002.

COSTA, G. R. M. da; COSTA, C. E. U.; SOUZA, A. M. Comparative studies of optimization methods for the optimal power flow problem. Electric Power Systems Research, v. 56, n. 3, p. 249-254, Nov. 2000.

DANIELS, R. L. Analytical evaluation of multi-criteria heuristics. Management Science, v. 38, p. 501-513, 1992.

DING, X.; WANG, X.; SONG, Y. H. Interior point cutting plane method for optimal power flow. IMA Journal of Management Mathematics, v. 15, n. 4, p. 355-368, 2004.

DOMMEL, H. W.; TINNEY, W. F. Optimal power flow solutions. IEEE Transactions on Power Apparatus and Systems, PAS-87, n. 10, p. 1866-1876, Oct. 1968.

DRUD, S. A. Conopt. Mathematical Programming, v. 31, p. 153-191, 1985.

FLETCHER, R.; POWELL, M. J. D. A rapidly convergent descent method for minimization. Computer Journal, v. 6, p. 163-168, 1963.

FRANK, S.; STEPONAVICE, I.; REBENNACK, S. Optimal power flow: a bibliographic survey I - formulations and deterministic methods. Energy System, v. 3, p. 221-258, 2012.

FRANK, S.; STEPONAVICE, I.; REBENNACK, S. Optimal power flow: a bibliographic survey II - non-deterministic and hybrid methods. Energy System, v. 3, p. 259-289, 2012.

GISVOLD, K. M.; MOE, J. A method for nonlinear mixed-integer programming and its application to design problems. Journal of Engineering for Industry, p. 353-364, 1972.

GRANVILLE, S. Optimal reactive dispatch through interior point methods. IEEE Transactions on Power Systems, v. 9, n. 1, p. 136-146, Feb. 1994.

GROSSMANN, I. E.; VISWANATHAN, J.; VECCHIETTI, A.; RAMAN, R.; KALVELAGEN, E. GAMS/DICOPT: A Discrete Continuous Optimization Package. [S.l.], 2002.

HUNEAUlT, M.; GALIANA, F. D. A survey of the optimal power flow literature. IEEE Transactions on Power Systems, v. 6, n. 2, p. 762-770, May 1991.

JEYANTHY, P. A.; DEVARAJ, D. Multi-objective genetic algorithm for reactive power optimisation including voltage stability. International Journal of Engineering Science and Technology, v. 2, n. 7, p. 2715-2729, 2010. 
JUBRIL, A.; KOMOLAFE, O.; ALAWODE, K. Solving multi-objective economic dispatch problem via semidefinite programming. Power Systems, IEEE Transactions on, v. 28, n. 3, p. 2056-2064, 2013.

LAGE, G. G. O Fluxo de Potência Ótimo Reativo com Variáveis de Controle Discretas e Restrições de Atuação de Dispositivos de controle de Tensão. Tese (Doutorado) - Escola de Engenharia de São Carlos - Universidade de São Paulo, 2013.

LIN, S.-Y.; HO, Y.-C.; LIN, C.-H. An ordinal optimization theory-based algorithm for solving the optimal power flow problem with discrete control variables. IEEE Transactions on Power Systems, v. 19, n. 1, p. 276-286, Feb. 2004.

LIU, M.; TSO, S. K.; CHENG, Y. An extended nonlinear primal-dual interior-point algorithm for reactive-power optimization of large-scale power systems with discrete control variables. IEEE Transactions on Power Systems, v. 17, n. 4, p. 982-991, Nov. 2002.

LIU, M. B.; CAÑIZARES, C. A.; HUANG, W. Reactive power and voltage control in distribution systems with limited switching operations. IEEE Transactions on Power Systems, v. 24, n. 2, p. 889-899, May 2009.

LIU, W.-H. E.; GUAN, X. Fuzzy constrait enforcement and control actions curtailment in an optimal power flow. IEEE Transactions Power Systems, v. 11, p. 639-645, 1996.

LiU, W.-H. E.; PAPAleXOPOUlOS, A. D.; TINNEY, W. F. Discrete shunt controls in a Newton optimal power flow. IEEE Transactions on Power Systems, v. 7, n. 4, p. 1509 -1518, Nov. 1992.

MAZZINI, A. P.; ASADA, E. N.; LAGE, G. G. The optimal reactive dispatch problem with discrete control variables and limited control adjustments. In: 2015 IEEE PES GENERAL MEETING, 2015, Denver. Proceedings... Denver: IEEE, 2015. v. 2, p. 1-5.

MIETTINEN, K. Nonlinear Multiobjective Optimization. 1st. ed. [S.1.]: Kluwer Academic Publishers, 1999.

MOLZAHN, D.; HOLZER, J.; LESIEUTRE, B.; DEMARCO, C. Implementation of a large-scale optimal power flow solver based on semidefinite programming. Power Systems, IEEE Transactions on, v. 28, n. 4, p. 3987-3998, Nov 2013.

MOLZAHN, D.; LESIEUTRE, B.; DEMARCO, C. A sufficient condition for global optimality of solutions to the optimal power flow problem. Power Systems, IEEE Transactions on, v. 29, n. 2, p. 978-979, March 2014.

MOMOH, J. A.; EL-HAWARY, M. E.; ADAPA, R. A review of selected optimal power flow literature to 1993: Part I: nonlinear and quadratic programming approaches. IEEE Transactions on Power Systems, v. 14, n. 1, p. 96-104, Feb. 1999a. 
MOMOH, J. A.; EL-HAWARY, M. E.; ADAPA, R. A review of selected optimal power flow literature to 1993: Part II: Newton, linear programming and interior point methods. IEEE Transactions on Power Systems, v. 14, n. 1, p. 105-111, Feb. 1999b.

MONTOYA, F. G.; BAñOS, R.; GIL, C.; ESPíN, A.; ALCAYDE, A.; GóMEZ, J. Minimization of voltage deviation and power losses in power networks using pareto optimization methods. Engineering Applications of Artificial Intelligence, v. 23, n. 5, p. 695-703, 2010.

MOTA-PALOMINO, R.; QUINTANA, V. Sparse reactive power scheduling by a penalty function - linear programming technique. Power Systems, IEEE Transactions on, v. 1, n. 3, p. 31-39, 1986.

MURRAY, W.; NG, K.-M. An algorithm for nonlinear optimization problems with binary variables. Springer Science, p. 257-288, 2008.

NIKNAM, T.; NARIMANI, M.; AZIZIPANAH-ABARGHOOEE, R.; BAHMANI-FIROUZI, B. Multiobjective optimal reactive power dispatch and voltage control: A new opposition-based self-adaptive modified gravitational search algorithm. Systems Journal, IEEE, v. 7, n. 4, p. 742-753, Dec 2013.

OLIVEIRA, A. R. L. de; PROBST, R. W. Novo método preditor-corretor para fluxo de potência Ótimo. In: XVIII CONGRESSO BRASILEIRO DE AUTOMáTICA, 2010, Bonito. Bonito: CBA, 2010. p. 578-583.

OLIVEIRA, E. J. de; JR., I. C. da S.; PEREIRA, J. L. R.; JR., S. C. Transmission system expansion planning using a sigmoid function to handle integer investment variables. IEEE Transactions on Power Systems, v. 20, n. 3, p. 1616-1621, 2005.

PANDYA, K. S.; JOSHI, S. K. A survey of optimal power flow methods. Journal of Theoretical E Applied Information Technology, v. 4, p. 450-458, 2008.

POWELL, M. J. D. An efficient method for finding the minimum of a function of several variables without calculating derivatives. Computer Journal, v. 7, p. 155-162, 1964.

QUINTANA, V. H.; TORRES, G. L.; MEDINA-PALOMO, J. Interior-point methods and their applications to power systems: a classification of publications and software codes. IEEE Transactions on Power Systems, v. 15, n. 1, p. 170-176, Feb. 2000.

RASHED, A. M. H.; KELLY, D. H. Optimal load flow solution using lagrangian multipliers and the hessian matrix. IEEE Transactions on Power Apparatus and Systems, PAS-93, n. 5, p. 1292-1297, Sept. 1974.

RIDER, M. J.; CASTRO, C. A.; BEDRIÑANA, M. F.; GARCIA, A. V. Towards a fast and robust interior point method for power system applications. IEEE Proceedings - Generation, Transmission and Distribution, v. 151, n. 5, p. 575-581, Sept. 2004.

SANTOS JR., A.; DECKMANN, S.; SOARES, S. A dual augmented lagrangian approach for optimal power flow. IEEE Transactions on Power Systems, v. 3, n. 3, p. 1020-1025, Aug. 1988. 
SASSON, A. M. Combined use of the Powell and Fletcher-Powell nonlinear programming methods for optimal load flows. IEEE Transactions on Power Apparatus and Systems, PAS-88, n. 10 , p. 1530-1537, Oct. 1969.

SASSON, A. M.; VILORIA, F.; ABOYTES, F. Optimal load flow solution using the Hessian matrix. IEEE Transactions on Power Apparatus and Systems, PAS-92, n. 1, p. 31-41, Jan. 1973.

SOLER, E. M.; ASADA, E. N.; COSTA, G. R. M. da. Penalty-based nonlinear solver for optimal reactive power dispatch with discrete controls. IEEE Transactions on Power Systems, v. 28, p. 2174-2182, 2013.

SOlER, E. M.; SOUSA, V. A. de; COSTA, G. R. M. da. A modified primal-dual logarithmic-barrier method for solving the optimal power flow problem with discrete and continuous control variables. European Journal of Operational Research, v. 222, n. 3, p. 616-622, 2012.

SOMAN, S. A.; PARTHASARATHY, K.; THUKARAM, D. Curtailed number and reduced controller movement optimization algorithms for real time voltage/reactive rower control. IEEE Transactions Power Systems, v. 9, p. 2035-2041, 1994.

SOUSA, V. A.; NUNES, L.; BELATI, E. A.; COSTA, G. A new approach type-newton for optimal reactive dispatch problem. Power Engineering Society General Meeting, p. 252-257, 2003.

SOUSA, V. A. de; BAPTISTA, E. C.; COSTA, G. R. M. da. Modified barrier method for optimal power flow problem. In: IEEE POWER ENGINEERING SOCIETY GENERAL MEETING, 2004, Denver. Proceedings... Denver: IEEE, 2004.

SOUSA, V. A. de; BAPTISTA, E. C.; COSTA, G. R. M. da. Loss minimization by a predictor-corrector modified approach. Electric Power Systems Research, v. 79, n. 5, p. 803-808, May 2009.

SOUSA, V. de; BAPTISTA, E.; COSTA, G. da. Optimal reactive power flow via the modified barrier lagrangian function approach. Electric Power Systems Research, v. 84, p. 159-164, 2012.

STEPHEN, D. S.; SOMASUNDARAM, P. Solution for multi-objective reactive power optimization using fuzzy guided tabu search. Arabian Journal for Science and Engineering, v. 37, n. 8, p. 2231-2241, 2012.

SUN, D. I.; ASHLEY, B.; BREWER, B.; HUGHES, A.; TINNEY, W. F. Optimal power flow by Newton approach. IEEE Transactions on Power Apparatus and Systems, PAS-103, n. 10, p. 2864-2880, Oct. 1984.

TINNEY, W. F.; BRIGHT, J. M.; DEMAREE, K. D.; HUGHES, B. A. Some deficiencies in optimal power flow. IEEE Transactions on Power Systems, v. 3, n. 2, p. 676-683, 1988. 
TORRES, G. L.; QUINTANA, V. H. An interior point method for nonlinear optimal power flow using voltage rectangular coordinates. IEEE Transactions on Power Systems, v. 13, n. 4, p. 1211-1218, Nov. 1998.

VARADARAJAN, M.; SWARUP, K. Solving multi-objective optimal power flow using differential evolution. IET Generation, Transmission and Distribution, v. 2, n. 5, p. 720-730, 2008.

WÄCHTER, A. An Interior Point Algorithm for Large-Scale Nonlinear Optimization with Applications in Process Engineering. Tese (Doutorado) - Carnegie Mellon University, 2002.

WÄCHTER, A. Short tutorial: Getting started with ipopt in 90 minutes. Combinatorial Scientific Computing, n. 09061, 2009.

WÄCHTER, A.; BIEGLER, L. T. On the implementation of a primal-dual interior point filter line search algorithm for large-scale nonlinear programming. Mathematical Programming, v. 106, n. 1 , p. $25-57,2006$.

WALTZ, R. A.; PLANTENGA, T. D. Knitro User's Manual. www.ziena.com, 2010. Version 7.0.

WOLF, P. Methods of nonlinear programming. Nonlinear Programming, 1967.

WU, Y.-C.; DEBS, A. S.; MARSTEN, R. E. A direct nonlinear predictor-corrector primal-dual interior point algorithm for optimal power flows. IEEE Transactions on Power Systems, v. 9, n. 2, p. 876-883, May 1994. 


\section{Apêndice A}

\section{Solvers e Métodos}

Neste Apêndice vamos apresentar uma breve descrição dos solvers CONOPT, IPOPT, KNITRO e DICOPT, utilizados neste trabalho e os métodos implementados em cada solver.

\section{A.1 CONOPT}

O solver CONOPT, é um solver comercial para problemas de otimização não linear de grande escala, desenvolvido e mantido por ARKI Consulting \& Development ${ }^{1}$ ao longo dos últimos 25 anos.

CONOPT é um solver de caminho viável baseado no método GRG que foi proposto por (ABADIE; CARPENTIER, 1969).O CONOPT foi projetado para ser eficiente e confiável para uma ampla classe de modelos. O método GRG original ajuda alcançar a viabilidade e velocidade para os modelos com um elevado grau de não-linearidade, ou seja, modelos difíceis. O solver CONOPT possui extensões para o método GRG como pré-processamento, uma fase especial 0 , iterações de modo linear, possui também uma programação linear sequencial e uma programação quadrática sequencial.

Um dos recursos mais importantes do CONOPT é uma implementação do componente de programação quadrática sequencial (PQS) que usa segundas derivadas exatas para calcular melhores direções de busca. O CONOPT usa um gradiente conjugado ou métodos de gradiente conjugado escalados para resolver os PQSs para modelos com muitas variáveis superbásicas ou para modelos com uma matriz bastante densa de segundas derivadas. O CONOPT pode utilizar as segundas derivadas de duas formas: como uma matriz esparsa de segundas derivados ou como o produto da matriz de segundas derivadas com uma direção de busca. O método escolhido depende da disponibilidade de memória e desempenho estatísticos.

A seguir, vamos apresentar algumas características básicas do método GRG juntamente com um algoritmo fundamental.

\footnotetext{
${ }^{1}$ http://www.conopt.com
} 


\section{A.1.1 Gradiente Reduzido Generalizado}

O método de gradiente reduzido foi proposto por (WOLF, 1967) para resolver problemas de PNL com restrições lineares. Posteriormente, (ABADIE; CARPENTIER, 1969) estenderam este método para o tratamento direto de restrições de desigualdade e restrições não lineares dando origem ao método de GRG. O método GRG particiona o conjunto das variáveis em variáveis básicas e não básicas. O método GRG modifica as restrições de modo que a mudança necessária nas variáveis básicas pode ser computada diretamente das variáveis não básicas, para isso, variáveis de folga são introduzidas para todas as restrições de desigualdade e as restrições não lineares são linearizadas no ponto de operação atual. Em seguida, o GRG é definido a partir da função objetivo que calcula a mudança incremental total na função objetivo considerando as duas variáveis básicas e não básicas.

Vamos apresentar a seguir os passos fundamentais de todo algoritmo GRG, para isto considere o seguinte problema PNL:

$$
\begin{array}{ll}
\min & x \\
\text { s.a: } & f(x)=b \\
& l \leq x \leq u
\end{array}
$$

onde $x \in \mathbb{R}^{m}$ é o vetor das variáveis de controle, $f: \mathbb{R}^{m} \rightarrow \mathbb{R}^{n} ; b \in \mathbb{R}^{n} ; l$ e $u$ são os limites inferiores e superiores das variáveis $x$, respectivamente.

A seguir apresentamos um algoritmo GRG com os passos básicos pra todos os algoritmos do tipo.

\section{Algoritmo GRG básico}

1. Inicializar e encontrar uma solução viável.

2. Calcule o Jacobiano das restrições, $J$.

3. Selecione um conjunto de $n$ variáveis básicas, $x_{b}$, de modo que $B$, a sub-matriz de coluna básica de $J$, é não singular. Fatore $B$. As demais variáveis, $x_{n}$, são chamados de não-básicas.

4. Resolva $B^{T} u=\frac{d f}{d x_{b}}$ para os multiplicadores $u$.

5. Calcule o gradiente reduzido, $r=\frac{d f}{d x}-J^{T} u$. Onde r é por definição zero para as variáveis básicas.

6. Se $r$ projetada sobre os limites é pequena, então pare. O ponto atual é próxima do ideal.

7. Selecione o conjunto de variáveis superbásicas, $x_{s}$, como um subconjunto de variáveis não-básicos que podem ser alterados de forma rentável, e encontrar uma direção de busca, 
$d_{s}$, para as variáveis superbásicas baseados em $r_{s}$ e, possivelmente, em algum segundo informações do pedido.

8. Faça uma pesquisa de linha ao longo da direção $d$. Para cada etapa, $x_{s}$ é alterado nos ds direção e $x_{b}$ é posteriormente ajustada para satisfazer $g\left(x_{b}, x_{s}\right)=b$ em um processo de pseudo-Newton usando o $B$ fatorado a partir do passo 3 .

9. Vá para 2 .

\section{A.2 IPOPT}

O IPOPT (Interior Point Optimizer) é um solver de código aberto para problemas PNL de grande escala, e faz parte do projeto COIN-OR (COmputational INfrastructure for Operational Research). O projeto COIN-OR é uma iniciativa para estimular o desenvolvimento de programas que auxiliem a comunidade científica que trabalha com Pesquisa Operacional. Os programas e códigos que fazem parte do projeto COIN-OR são distribuidos gratuitamente e podem ser encontrados na página do projeto http://www.coin-or.org.

O código do IPOPT foi escrito principalmente por Andreas Wächter, que é o líder do projeto COIN-OR para IPOPT. O IPOPT é uma implementação de um Algoritmo de Pontos Interiores com Filtro para modelos de programação não linear, onde as funções podem ser não convexas porém duplamente diferenciáveis.

Basicamente, dado um problema PNL, o método de Pontos Interiores com Filtro adiciona variáveis de folga ou excesso, não negativas, nas restrições de desigualdade do problema. As restrições de não negatividade das variáveis de folga são tratadas pela Função Barreira, obtendo assim um problema Barreira, somente com restrições de igualdade. Associa-se ao problema Barreira uma função Lagrangiana, e aplicam-se as Condições Necessárias de Primeira Ordem, obtendo um sistema não linear que é resolvido pelo método de Newton. As iterações do método de Newton fornecem direções de busca para atualização das variáveis. No cálculo do tamanho do passo utiliza-se uma busca unidimencional com Filtro. Detalhes sobre o método e a implementação podem ser encontrados em (WÄCHTER, 2002), (WÄCHTER; BIEGLER, 2006) e (WÄCHTER, 2009).

\section{A.3 KNITRO}

O KNITRO é um solver comercial para otimização não linear, desenvolvido e mantido por ZIENA Optimization LLC. KNITRO é projetado para problemas de grande escala, sendo eficaz para solução de problemas lineares, problemas de otimização quadrática suaves e não-lineares, tanto convexa e convexo. Também é eficaz para regressão não-linear, problemas com restrições de complementaridade e programação inteira mista (MIP), em particular problemas de otimização inteira mista convexo.

O KNITRO possui três algoritmos implementados: Algoritmo de Pontos Interiores Direto; Algoritmo de Pontos Interiores com Gradientes Conjugados; e Algoritmo de Conjuntos 
Ativos. O Algoritmo de Pontos Interiores Direto aplica técnicas de barreira e fatoriza a matriz KKT direta do sistema não linear. Possui o melhor desempenho entre os algoritmos do KNITRO para problemas mal condicionados. O Algoritmo de Pontos Interiores com Gradientes Conjugados aplica técnicas de barreira e utiliza o método do gradiente conjugado para resolver os subproblemas KKT. Ele fornece uma alternativa ao Algoritmo de Pontos Interiores Direto, quando a fatoração KKT é impraticável. O Algoritmo de Conjuntos Ativos combina princípios de conjuntos ativos clássicos com um novo subproblema de programação linear para descobrir rapidamente o conjunto de restrições de ligação. Seu comportamento é significativamente diferente dos algoritmos de pontos interiores, e converge justamente para o conjunto ativo fornecendo informações de alta precisão sensibilidade.

Para os problema de Programação Inteira Mista, o Knitro oferece duas variantes do algoritmo Branch-and-Bound. A primeira é uma implementação padrão, enquanto a segunda é especializada para problemas de Programação Não Linear Inteira Mista.

O KNITRO pode ser encontrado na página http://www.ziena.com/knitro.htm e maiores informações podem ser encontradas em (WALTZ; PLANTENGA, 2010).

\section{A.4 DICOPT}

O DICOPT é um solver para resolver problemas de PNLIM. O DICOPT (DIscrete and Continuous OPTimizer) foi desenvolvido por J. Viswanathan e Ignacio E. Grossmann na Engineering Design Research Center (EDRC) da Universidade de Carnegie Mellon. O solver baseia-se nas extensões do algoritmo outer-approximation para a estratégia de relaxamento das restrições de igualdade. O algoritmo DICOPT resolve uma série de subproblemas de PNL e PIM. Estes sub-problemas podem ser resolvidos usando qualquer algoritmo de PNL ou PIM que fazem parte da interface utilizada, no caso do GAMS, o DICOPT utiliza os pacotes já carregados pela interface.

O sistema GAMS/DICOPT foi projetado com dois objetivos principais em mente:

1) Construir sobre os conceitos de modelagem existentes e introduzindo o mínimo de extensões para a linguagem de modelagem existente e fornecer uma alta compatibilidade para garantir a fácil transição de aplicações de modelagem existentes para formulações não-lineares inteiros mistos.

2) Utilizar solvers existentes para resolver os sub-problemas criados pelo DICOPT. Isto permite combinar os melhores algoritmos para o problema e garante que qualquer novo desenvolvimento e melhorias na PNL e solucionadores de problemas de PIM se tornam automaticamente e imediatamente disponíveis para DICOPT.

Maiores informações sobre o DICOPT junto com a interface GAMS podem ser encontradas em (GROSSMANN et al., 2002). 


\section{Apêndice B}

\section{Fluxo de Potência Ótimo - Expressões Gerais dos Fluxos}

O problema de FPO com objetivo de minimizar as perdas ativas na transmissão pode ser representado como:

$$
\begin{array}{lll}
\min & \sum_{k, m \in \mathcal{L} \cup \mathcal{T}} g_{k m}\left(\frac{1}{t_{k m}^{2}} V_{k}^{2}+V_{m}^{2}-2 \frac{1}{t_{k m}} V_{k} V_{m} \cos \theta_{k m}\right) & \\
\text { s.a: } & P_{k}-\sum_{m \in \mathcal{V}_{k}} P_{k m}(V, \theta, t)=0 & \forall k \in \mathcal{G}^{\prime} \cup \mathcal{C} \\
& Q_{k}+Q_{k}^{s h}\left(V_{k}, b_{k}^{s h}\right)-\sum_{m \in \mathcal{V}_{k}} Q_{k m}(V, \theta, t)=0 & \forall k \in \mathcal{C} \\
& Q_{G_{k}}^{\text {min }} \leq Q_{G_{k}}\left(V, \theta, t, b_{k}^{s h}\right) \leq Q_{G_{k}}^{\text {max }} & \forall k \in \mathcal{G} \\
V_{k}^{\text {min }} \leq V_{k} \leq V_{k}^{\text {max }} & \forall k \in \mathcal{B} \\
b_{k}^{s h} \in \mathcal{D}_{k}^{s h} & \forall k \in \mathcal{B}^{s h} \\
t_{k m} \in \mathcal{D}_{k m}^{\text {tap }} & \forall k, m \in \mathcal{T}
\end{array}
$$

onde $V$ e $\theta$ são, respectivamente, os vetores das magnitudes e ângulos de fase da tensão nas barras do sistema; $t$ é o vetor dos taps variáveis de transformadores em-fase; $b^{\text {sh }}$ é o vetor das susceptâncias equivalentes dos bancos de capacitores e reatores shunt; $P_{k}$ e $Q_{k}$ representam, respectivamente, as injeções líquidas de potência ativa e reativa na barra $k ; Q_{G_{k}}$ é a potência reativa gerada na barra $k ; Q_{k}^{s h}$ é a injeção de potência reativa pelo shunt da barra $k ; P_{k m}$ e $Q_{k m}$ são os fluxos de potência ativa e reativa no ramo $k-m ; \theta_{k m}$ é a diferença entre os ângulos de fase da tensão nas barras $k$ e $m$; e $g_{k m}$ é a condutância série associada ao elemento de transmissão do ramo $k-m$.

Ainda, tem-se que $\mathcal{B}$ é o conjunto de todas as barras do sistema; $\mathcal{G}$ é conjunto das barras de geração; $\mathcal{G}^{\prime}$ é conjunto das barras de geração menos a barra slack; $\mathcal{C}$ é conjunto das barras de 
carga; $\mathcal{B}^{\text {sh }}$ é conjunto das barras com controle da magnitude de tensão por bancos de capacitores e reatores shunt; $\mathcal{L}$ é o conjunto dos ramos $k-m$ que representam linhas de transmissão; $\mathcal{T}$ é conjunto dos ramos $k-m$ que representam transformadores em-fase com tap variável; $\mathcal{V}_{k}$ é o conjunto das barras vizinhas à barra $k ; \mathcal{D}_{k m}^{\text {tap }}$ é o conjunto dos valores discretos que os taps dos transformadores podem assumir, e $\mathcal{D}_{k}^{s h}$ é o conjunto dos valores discretos que as susceptâncias equivalentes aos bancos de capacitores e reatores shunts podem assumir.

As expressões dos fluxos de potência ativa e reativa em linhas de transmissão e transformadores, para o caso em que a barra $k$ é a barra do tap e a barra $m$ é a barra da impedância, são dadas por:

$$
\begin{gathered}
P_{k m}(V, \theta, t)=g_{k m} \frac{1}{t_{k m}^{2}} V_{k}^{2}-\frac{1}{t_{k m}} V_{k} V_{m}\left[g_{k m} \cos \left(\theta_{k m}\right)+b_{k m} \sin \left(\theta_{k m}\right)\right] \\
Q_{k m}(V, \theta, t)=-\left(b_{k m} \frac{1}{t_{k m}^{2}}+b_{k m}^{s h}\right) V_{k}^{2}+\frac{1}{t_{k m}} V_{k} V_{m}\left[b_{k m} \cos \left(\theta_{k m}\right)-g_{k m} \sin \left(\theta_{k m}\right)\right] \\
P_{m k}(V, \theta, t)=g_{k m} V_{m}^{2}-\frac{1}{t_{k m}} V_{k} V_{m}\left[g_{k m} \cos \left(\theta_{k m}\right)-b_{k m} \sin \left(\theta_{k m}\right)\right] \\
Q_{m k}(V, \theta, t)=-\left(b_{k m}+b_{k m}^{s h}\right) V_{m}^{2}+\frac{1}{t_{k m}} V_{k} V_{m}\left[b_{k m} \cos \left(\theta_{k m}\right)+g_{k m} \sin \left(\theta_{k m}\right)\right]
\end{gathered}
$$

Para as linhas de transmissão, $t_{k m}=0$; para os transformadores em-fase $b_{k m}^{s h}=0$; e para defasadores, $b_{k m}^{s h}=0$ e $t_{k m}=1$.

A geração de potência reativa injetada nas barras de controle reativo é modelada como:

$$
Q_{k}\left(V, \theta, t, b^{s h}\right)=-\left(b_{k m} \frac{1}{t_{k m}^{2}}+b_{k m}^{s h}\right) V_{k}^{2}+\frac{1}{t_{k m}} V_{k} V_{m}\left[b_{k m} \cos \left(\theta_{k m}\right)-g_{k m} \sin \left(\theta_{k m}\right)\right]
$$

